Supporting Information for

\title{
A Synthetic Strategy toward Eight-Membered Cyclic Amines by Cycloetherification and Claisen Rearrangement
}

\author{
Seo-Jung Han, ${ }^{\dagger, \#,+}$ Marchello A. Cavitt, ${ }^{\dagger \neq}$ and Brian M. Stoltz ${ }^{*, \dagger}$ \\ tThe Warren and Katharine Schlinger Laboratory for Chemistry and Chemical \\ Engineering, Division of Chemistry and Chemical Engineering, California Institute of \\ Technology, 1200 East California Boulevard, MC 101-20, Pasadena, California 91125, \\ United States \\ ${ }^{\#}$ Chemical Kinomics Research Center, KIST and Division of Bio-Medical Science \& \\ Technology, KIST-School UST, 5, Hwarangro 14-gil, Seongbuk-gu, Seoul, 02792, \\ Republic of Korea
}

stoltz@caltech.edu

\section{Table of Contents}

Materials and Methods

SI-2

Experimental Procedures and Spectroscopic Data

SI-4

${ }^{1} \mathrm{H}$ NMR and ${ }^{13} \mathrm{C}$ NMR Spectra

SI-33 


\section{Materials and Methods}

Unless otherwise stated, reactions were performed in flame-dried glassware under an argon or nitrogen atmosphere using dry, deoxygenated solvents. Reaction progress was monitored by thin-layer chromatography (TLC). THF, $\mathrm{Et}_{2} \mathrm{O}, \mathrm{CH}_{2} \mathrm{Cl}_{2}$, toluene, benzene, $\mathrm{CH}_{3} \mathrm{CN}$, and dioxane were dried by passage through an activated alumina column under argon. Purified water was obtained using a Barnstead NANOpure Infinity UV/UF system. Brine solutions are saturated aqueous solutions of sodium chloride. Commercially available reagents were purchased from Sigma-Aldrich, Acros Organics, Strem, or Alfa Aesar and used as received unless otherwise stated. Reaction temperatures were controlled by an IKAmag temperature modulator unless otherwise indicated. Glove box manipulations were performed under a $\mathrm{N}_{2}$ atmosphere. TLC was performed using E. Merck silica gel 60 F254 precoated glass plates $(0.25 \mathrm{~mm})$ and visualized by UV fluorescence quenching, $p$-anisaldehyde, $\mathrm{KMnO}_{4}$ or $\mathrm{PMA}$ (phosphomolybdic acid) staining. Silicycle SiliaFlash P60 Academic Silica gel (particle size $0.040-0.064 \mathrm{~mm}$ ) was used for flash column chromatography. ${ }^{1} \mathrm{H}$ NMR spectra were recorded on a Varian Inova $300 \mathrm{MHz}, 500 \mathrm{MHz}$ and $600 \mathrm{MHz}$ and Bruker $400 \mathrm{MHz}$ spectrometers. The ${ }^{1} \mathrm{H}$ NMR spectra are reported relative to residual $\mathrm{CHCl}_{3}(\delta 7.26 \mathrm{ppm}), \mathrm{C}_{6} \mathrm{D}_{6}(\delta 7.16 \mathrm{ppm})$ or $\mathrm{CD}_{3} \mathrm{OD}(\delta 3.31 \mathrm{ppm}) .{ }^{13} \mathrm{C}$ NMR spectra are recorded on a Varian Inova $300 \mathrm{MHz}(75 \mathrm{MHz})$ and $500 \mathrm{MHz}$ spectrometer (125 $\mathrm{MHz}$ ) and Bruker $400 \mathrm{MHz}$ spectrometers $(100 \mathrm{MHz})$. The ${ }^{13} \mathrm{C}$ NMR spectra are reported relative to $\mathrm{CHCl}_{3}(\delta 77.16 \mathrm{ppm}), \mathrm{C}_{6} \mathrm{D}_{6}(\delta 128.06 \mathrm{ppm})$, or $\mathrm{CD}_{3} \mathrm{OD}(\delta 49.01$ ppm). Data for ${ }^{1} \mathrm{H}$ NMR are reported as follows: chemical shift ( $\left.\delta \mathrm{ppm}\right)$ (multiplicity, coupling constant (Hz), integration). Multiplicities are reported as follows: $\mathrm{s}=$ singlet, $\mathrm{d}=$ doublet, $\mathrm{t}=$ triplet, $\mathrm{q}=$ quartet, $\mathrm{p}=$ pentet, $\mathrm{sept}=$ septuplet, $\mathrm{m}=$ multiplet, $\mathrm{br} \mathrm{s}$ $=$ broad singlet, br $\mathrm{d}=$ broad doublet, app = apparent. Data for ${ }^{13} \mathrm{C} \mathrm{NMR}$ are reported 
in terms of chemical shifts $(\delta \mathrm{ppm})$. IR spectra were obtained using a Perkin Elmer Paragon 1000 spectrometer using thin films deposited on $\mathrm{NaCl}$ plates and reported in frequency of absorption $\left(\mathrm{cm}^{-1}\right)$. High resolution mass spectra (HRMS) were obtained from the Caltech Mass Spectral Facility using JEOL JMS-600H High Resolution Mass Spectrometer in fast atom bombardment $(\mathrm{FAB}+)$ or electron ionization (EI+) mode, or using an Agilent 6200 Series TOF with an Agilent G1978A Multimode source in electrospray ionization (ESI+), atmospheric pressure chemical ionization (APCI+), or mixed ionization mode (MM: ESI-APCI+). 


\section{Representative procedure for aminoalcohols 1}

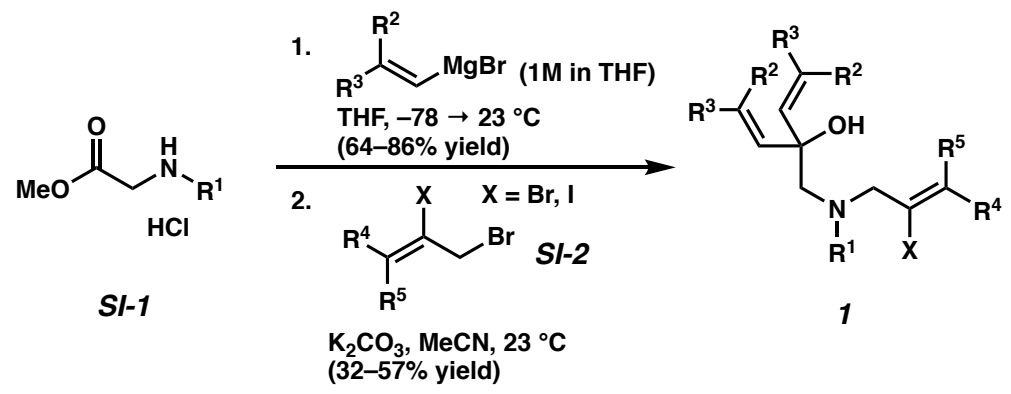

To a solution of ester SI-1 (3.58 mmol, 1.00 equiv) in THF (18.0 mL) was added vinylmagnesium bromide ( $1 \mathrm{M}$ in THF) $\left(17.9 \mathrm{mmol}, 5.00\right.$ equiv) dropwise at $-78{ }^{\circ} \mathrm{C}$. The solution was stirred for $12 \mathrm{~h}$ at $23{ }^{\circ} \mathrm{C}$. The solution was quenched with sat. $\mathrm{NH}_{4} \mathrm{Cl}$ at $0{ }^{\circ} \mathrm{C}$. The aqueous phase was washed with $\mathrm{Et}_{2} \mathrm{O}(3 \times 20.0 \mathrm{~mL})$. The combined organic phase was washed with brine, dried over anhydrous $\mathrm{MgSO}_{4}$, and concentrated in vacuo. The residue was purified by flash column chromatography on silica gel to give tertiary alcohols.

To a solution of tertiary alcohol (1.38 mmol, 1.00 equiv) in $\mathrm{MeCN}(6.90 \mathrm{~mL})$ were added $\mathrm{K}_{2} \mathrm{CO}_{3}$ (6.90 mmol, 5.00 equiv) and allyl bromide SI-2 (1.45 mmol, 1.05 equiv). The solution was stirred for $12 \mathrm{~h}$ at $23^{\circ} \mathrm{C}$. After the reaction was completed, water was added. The aqueous phase was washed with EtOAc $(3 \times 6.00 \mathrm{~mL})$. The combined organic phases were washed with brine, dried over anhydrous $\mathrm{MgSO}_{4}$, and concentrated in vacuo. The residue was purified by flash column chromatography on silica gel to give aminoalcohols $\mathbf{1}$.

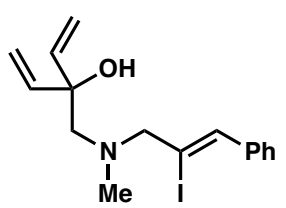

$1 b$ 
1b (178 mg, $0.482 \mathrm{mmol}$ ) was synthesized from sarconic methyl ester hydrochloride (193 mg, $1.38 \mathrm{mmol}) ; 35 \%$ yield (2 steps); $\mathrm{R}_{f}=0.25$ (1:8 EtOAc:hexanes); ${ }^{1} \mathrm{H}$ NMR $\left(400 \mathrm{MHz}, \mathrm{CDCl}_{3}\right) \delta 7.65-7.48(\mathrm{~m}, 2 \mathrm{H}), 7.41-7.31(\mathrm{~m}, 3 \mathrm{H}), 6.96(\mathrm{~s}, 1 \mathrm{H}), 5.93(\mathrm{dd}$, $J=17.3,10.6 \mathrm{~Hz}, 2 \mathrm{H}), 5.41(\mathrm{~d}, J=17.3 \mathrm{~Hz}, 2 \mathrm{H}), 5.16(\mathrm{~d}, J=10.7 \mathrm{~Hz}, 2 \mathrm{H}), 4.14(\mathrm{~s}, \mathrm{br}$, 1H), 3.45 (s, 2H), 2.69 (s, 2H), $\left.2.32(\mathrm{~s}, 3 \mathrm{H}) ;{ }^{13} \mathrm{C} \mathrm{NMR} \mathrm{(101} \mathrm{MHz,} \mathrm{CDCl}_{3}\right) \delta$ 142.0, 137.4, 136.2, 128.8, 128.8, 128.3, 114.0, 107.4, 74.5, 71.8, 66.0, 43.1; IR (Neat Film $\mathrm{NaCl})$ 2846, 2792, 2361, 2343, 1447, 999, 921, 750, $695 \mathrm{~cm}^{-1}$; HRMS (MM: ESIAPCI+) m/z calc'd for $\mathrm{C}_{16} \mathrm{H}_{21} \mathrm{OIN}[\mathrm{M}+\mathrm{H}]^{+}: 370.0662$; found: 370.0688 .

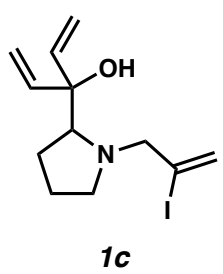

1c $(117 \mathrm{mg}, 0.367 \mathrm{mmol})$ was synthesized from proline methyl ester hydrochloride (145 mg, $0.875 \mathrm{mmol}) ; 42 \%$ yield (2 steps); $\mathrm{R}_{f}=0.55$ (1:8 EtOAc:hexanes); ${ }^{1} \mathrm{H}$ NMR $\left(500 \mathrm{MHz}, \mathrm{CDCl}_{3}\right) \delta 6.28(\mathrm{dt}, J=2.1,1.1 \mathrm{~Hz}, 1 \mathrm{H}), 5.93(\mathrm{dd}, J=17.2,10.6 \mathrm{~Hz}, 1 \mathrm{H})$, $5.86(\mathrm{dd}, J=17.3,10.8 \mathrm{~Hz}, 1 \mathrm{H}), 5.80(\mathrm{t}, J=1.5 \mathrm{~Hz}, 1 \mathrm{H}), 5.45(\mathrm{dd}, J=17.2,1.5 \mathrm{~Hz}$, 1H), $5.32(\mathrm{dd}, J=17.3,1.6 \mathrm{~Hz}, 1 \mathrm{H}), 5.12(\mathrm{ddd}, J=11.5,10.7,1.6 \mathrm{~Hz}, 2 \mathrm{H}), 3.75(\mathrm{dt}, J$ $=14.2,1.8 \mathrm{~Hz}, 1 \mathrm{H}), 3.60(\mathrm{~s}, 1 \mathrm{H}), 3.08-3.00(\mathrm{~m}, 1 \mathrm{H}), 2.95-2.85(\mathrm{~m}, 2 \mathrm{H}), 2.33-2.22$ $(\mathrm{m}, 1 \mathrm{H}), 1.92-1.81(\mathrm{~m}, 1 \mathrm{H}), 1.83-1.53(\mathrm{~m}, 4 \mathrm{H}) ;{ }^{13} \mathrm{C} \mathrm{NMR}\left(126 \mathrm{MHz}, \mathrm{CDCl}_{3}\right) \delta$ 142.6, 140.1, 125.7, 113.7, 113.2, 112.4, 69.6, 67.8, 54.6, 27.9, 24.5; IR (Neat Film $\mathrm{NaCl})$ 2964, 2871, 2799, 1617, 1409, 1355, 1114, 998, $920 \mathrm{~cm}^{-1}$; HRMS (MM: ESIAPCI+) m/z calc'd for $\mathrm{C}_{12} \mathrm{H}_{19} \mathrm{ONI}[\mathrm{M}+\mathrm{H}]^{+}:$320.0506; found: 320.0512 .

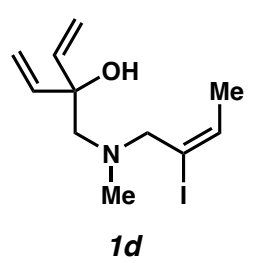


1d $(88.8 \mathrm{mg}, 0.289 \mathrm{mmol})$ was synthesized from sarconic methyl ester hydrochloride (115 mg, 0.826 mmol); 35\% yield (2 steps); $\mathrm{R}_{f}=0.35$ (1:8 EtOAc:hexanes); ${ }^{1} \mathrm{H}$ NMR $\left(400 \mathrm{MHz}, \mathrm{CDCl}_{3}\right) \delta 6.42(\mathrm{qt}, J=7.2,1.2 \mathrm{~Hz}, 1 \mathrm{H}), 5.92(\mathrm{~d}, J=10.7 \mathrm{~Hz}, 1 \mathrm{H}), 5.87$ (d, $J=10.7 \mathrm{~Hz}, 2 \mathrm{H}), 5.39(\mathrm{dd}, J=17.2,1.5 \mathrm{~Hz}, 2 \mathrm{H}), 5.13(\mathrm{dd}, J=10.6,1.5 \mathrm{~Hz}, 2 \mathrm{H}), 4.17$ (s, 1H), $3.16(\mathrm{~s}, 2 \mathrm{H}), 2.62(\mathrm{~s}, 2 \mathrm{H}), 2.24(\mathrm{~s}, 3 \mathrm{H}), 1.71(\mathrm{dt}, J=7.0,0.8 \mathrm{~Hz}, 3 \mathrm{H}) ;{ }^{13} \mathrm{C} \mathrm{NMR}$ $\left(101 \mathrm{MHz}, \mathrm{CDCl}_{3}\right) \delta 142.1,138.7,113.9,102.8,74.4,66.0,62.3,43.0,17.1$; IR (Neat Film NaCl) 2948, 2846, 1452, 1303, 1112, 1039, 996, $922 \mathrm{~cm}^{-1}$; HRMS (MM: ESIAPCI+) m/z calc'd for $\mathrm{C}_{11} \mathrm{H}_{19} \mathrm{ONI}[\mathrm{M}+\mathrm{H}]^{+}: 308.0506$; found: 308.0517 .

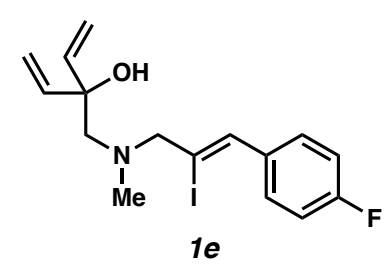

1e (158 mg, $0.408 \mathrm{mmol})$ was synthesized from sarconic methyl ester hydrochloride (130 mg, $0.928 \mathrm{mmol}) ; 44 \%$ yield (2 steps); $\mathrm{R}_{f}=0.35$ (1:8 EtOAc:hexanes); ${ }^{1} \mathrm{H}$ NMR $\left(400 \mathrm{MHz}, \mathrm{CDCl}_{3}\right) \delta 7.55-7.44(\mathrm{~m}, 2 \mathrm{H}), 7.10-6.99(\mathrm{~m}, 2 \mathrm{H}), 6.91(\mathrm{~s}, 1 \mathrm{H}), 5.92(\mathrm{dd}$, $J=17.3,10.7 \mathrm{~Hz}, 2 \mathrm{H}), 5.40(\mathrm{dd}, J=17.3,1.5 \mathrm{~Hz}, 2 \mathrm{H}), 5.15(\mathrm{dd}, J=10.7,1.5 \mathrm{~Hz}, 2 \mathrm{H})$, $4.09(\mathrm{~s}, 1 \mathrm{H}), 3.42(\mathrm{~s}, 2 \mathrm{H}), 2.69(\mathrm{~s}, 2 \mathrm{H}), 2.31(\mathrm{~s}, 3 \mathrm{H}) ;{ }^{13} \mathrm{C} \mathrm{NMR}\left(101 \mathrm{MHz}, \mathrm{CDCl}_{3}\right) \delta$ $162.5(\mathrm{~d}, J=248.2 \mathrm{~Hz}), 142.0,135.0,133.4(\mathrm{~d}, J=3.4 \mathrm{~Hz}), 130.7(\mathrm{~d}, J=8.1 \mathrm{~Hz}), 115.3$ $(\mathrm{d}, J=21.6 \mathrm{~Hz}), 114.0,107.7,74.6,71.8,66.0,43.2 ;{ }^{19} \mathrm{~F} \mathrm{NMR}\left(282 \mathrm{MHz}, \mathrm{CDCl}_{3}\right) \delta-$ 112.9; IR (Neat Film NaCl) 2848, 2360, 2342, 1601, 1506, 1227, 1158, 997, $923 \mathrm{~cm}^{-1}$; HRMS (MM: ESI-APCI+) m/z calc'd for $\mathrm{C}_{16} \mathrm{H}_{20} \mathrm{ONIF}[\mathrm{M}+\mathrm{H}]^{+}:$388.0568; found: 388.0578 .

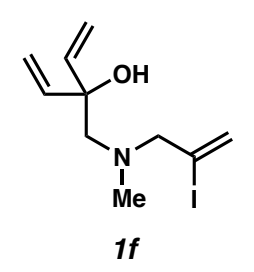


1f $(61.3 \mathrm{mg}, 0.209 \mathrm{mmol})$ was synthesized from sarconic methyl ester hydrochloride (122 mg, $0.873 \mathrm{mmol}) ; 24 \%$ yield (2 steps); $\mathrm{R}_{f}=0.35$ (1:8 EtOAc:hexanes); ${ }^{1} \mathrm{H}$ NMR $\left(400 \mathrm{MHz}, \mathrm{CDCl}_{3}\right) \delta 6.27(\mathrm{q}, J=1.4 \mathrm{~Hz}, 1 \mathrm{H}), 5.92(\mathrm{~d}, J=10.6 \mathrm{~Hz}, 2 \mathrm{H}), 5.92-5.83$ (m, 3H), $5.39(\mathrm{dd}, J=17.3,1.5 \mathrm{~Hz}, 2 \mathrm{H}), 5.14(\mathrm{dd}, J=10.7,1.5 \mathrm{~Hz}, 2 \mathrm{H}), 4.03(\mathrm{~s}, 1 \mathrm{H})$, $3.14(\mathrm{~s}, 2 \mathrm{H}), 2.63(\mathrm{~s}, 2 \mathrm{H}), 2.26(\mathrm{~s}, 3 \mathrm{H}) ;{ }^{13} \mathrm{C} \mathrm{NMR}\left(101 \mathrm{MHz}, \mathrm{CDCl}_{3}\right) \delta$ 141.9, 127.3, 114.0, 111.9, 74.4, 70.0, 66.0, 43.2; IR (Neat Film NaCl) 2917, 2848, 2791, 1617, 1451, 1406, 1304, 1154, 1117, 1038, 996, $992 \mathrm{~cm}^{-1}$; HRMS (MM: ESI-APCI+) m/z calc'd for $\mathrm{C}_{10} \mathrm{H}_{17} \mathrm{ONI}[\mathrm{M}+\mathrm{H}]^{+}$: 294.0349; found: 294.0364 .

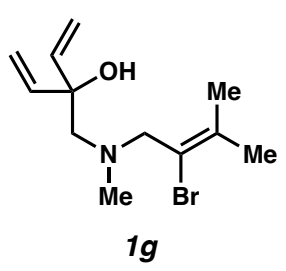

$1 \mathrm{~g}$ (106 mg, $0.385 \mathrm{mmol}$ ) was synthesized from sarconic methyl ester hydrochloride (110 mg, $0.786 \mathrm{mmol}) ; 49 \%$ yield (2 steps); $\mathrm{R}_{f}=0.35$ (1:8 EtOAc:hexanes); ${ }^{1} \mathrm{H}$ NMR $\left(500 \mathrm{MHz}, \mathrm{CDCl}_{3}\right) \delta 5.89(\mathrm{dd}, J=17.3,10.7 \mathrm{~Hz}, 2 \mathrm{H}), 5.37(\mathrm{dd}, J=17.3,1.6 \mathrm{~Hz}, 2 \mathrm{H})$, $5.12(\mathrm{dd}, J=10.6,1.5 \mathrm{~Hz}, 2 \mathrm{H}), 3.40(\mathrm{~s}, 2 \mathrm{H}), 2.60(\mathrm{~s}, 2 \mathrm{H}), 2.27(\mathrm{~s}, 3 \mathrm{H}), 1.91(\mathrm{~s}, 3 \mathrm{H})$, $1.83(\mathrm{~s}, 3 \mathrm{H}) ;{ }^{13} \mathrm{C} \mathrm{NMR}\left(126 \mathrm{MHz}, \mathrm{CDCl}_{3}\right) \delta 142.3,134.9,119.8,113.7,74.1,65.9$, 62.9, 43.0, 25.8, 21.2; IR (Neat Film NaCl) 2918, 2846, 1458, 1410, 1364, 1305, 1040, 998, $922 \mathrm{~cm}^{-1}$; HRMS (MM: ESI-APCI+) m/z calc'd for $\mathrm{C}_{12} \mathrm{H}_{21} \mathrm{ONBr}[\mathrm{M}+\mathrm{H}]^{+}$: 274.0801; found: 274.0826 .

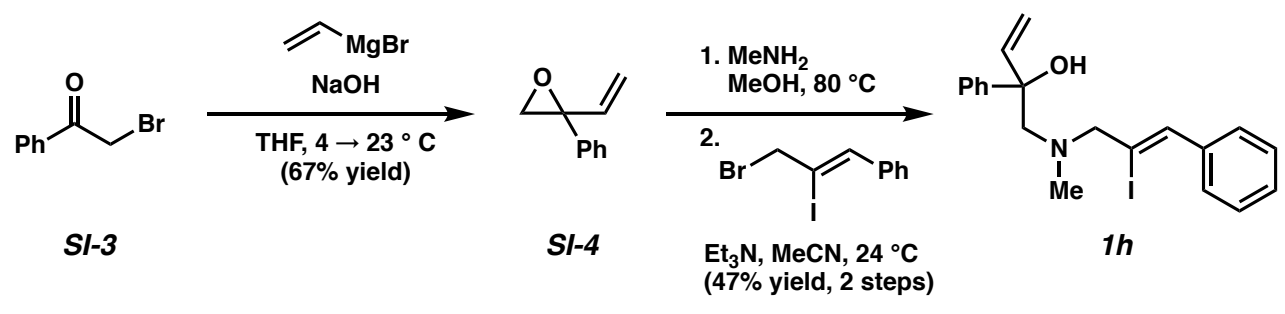

A $500 \mathrm{~mL}$ flame-dried round bottom flask, equipped with a stir bar, was placed under an argon atmosphere. Vinyl magnesium bromide (1 M in THF, $26.0 \mathrm{~mL}, 26.0 \mathrm{mmol})$ 
was charged to the reaction vessel. After cooling the solution for $10 \mathrm{~min}$ at $4{ }^{\circ} \mathrm{C}, 2$ bromo-1-phenylethanone $(3.50 \mathrm{~g}, 17.2 \mathrm{mmol})$ in THF $(88.0 \mathrm{~mL})$ was added slowly. Then, aq. $\mathrm{NaOH}(1 \mathrm{M}, 80.0 \mathrm{~mL}, 80.0 \mathrm{mmol})$ was poured into the reaction pot at $4{ }^{\circ} \mathrm{C}$. The reaction mixture was allowed to warm to $23{ }^{\circ} \mathrm{C}$ and stirred for $2 \mathrm{~h}$. Water $(50.0$ $\mathrm{mL})$ and EtOAc $(40.0 \mathrm{~mL})$ were added. Additional extract was obtained using EtOAc ( $2 \times 50 \mathrm{~mL})$. The combined organic layers were washed with brine $(250 \mathrm{~mL})$, dried over anhydrous sodium sulfate, filtered, and concentrated for purification. The residue was purified using basic alumina with EtOAc/Petroleum ether (1:50) to obtain 2phenyl-2-vinyloxirane (1.68 g, 67\% yield).

2-Phenyl-2-vinyloxirane ( $800 \mathrm{mg}, 5.47 \mathrm{mmol})$, methyl amine (2 $\mathrm{M}$ in THF, $3.60 \mathrm{~mL}$, $7.20 \mathrm{mmol})$, and $\mathrm{MeOH}(3.20 \mathrm{~mL})$ were combined in $20 \mathrm{~mL}$ vial with a stir bar. The vial was heated at $80{ }^{\circ} \mathrm{C}$ via heating block for $12 \mathrm{~h}$. The vial was allowed to cool to 23 ${ }^{\circ} \mathrm{C}$. Solvent was removed under reduced pressure and placed on high vacuum for 10 min to afford an aminoalcohol.

To the residue were added $\mathrm{MeCN}(8.00 \mathrm{~mL}), \mathrm{Et}_{3} \mathrm{~N}(2.00 \mathrm{~mL}, 14.0 \mathrm{mmol})$, and $(Z)-(3-$ bromo-2-iodoprop-1-en-1-yl)benzene (1.58 g, $4.89 \mathrm{mmol})$. After $11.5 \mathrm{~h}$, solvent was removed under reduced pressure and purified by silica gel chromatography to give (Z)1-((2-iodo-3-phenylallyl)(methyl)amino)-2-phenylbut-3-en-2-ol (1.07 g, 47\% 2-step yield) as an orange oil. ${ }^{1}$

$\mathrm{R}_{f}=0.59$ (1:4 EtOAc:hexanes); ${ }^{1} \mathrm{H}$ NMR $\left(500 \mathrm{MHz}, \mathrm{CDCl}_{3}\right) \delta 7.56-7.47(\mathrm{~m}, 4 \mathrm{H})$, $7.40-7.31(\mathrm{~m}, 5 \mathrm{H}), 7.27-7.22(\mathrm{~m}, 1 \mathrm{H}), 6.93(\mathrm{~s}, 1 \mathrm{H}), 6.21(\mathrm{dd}, J=17.1,10.6 \mathrm{~Hz}, 1 \mathrm{H})$, $5.46(\mathrm{dd}, J=17.0,1.5 \mathrm{~Hz}, 1 \mathrm{H}), 5.16(\mathrm{dd}, J=10.6,1.5 \mathrm{~Hz}, 1 \mathrm{H}), 4.74(\mathrm{~s}, 1 \mathrm{H}), 3.43(\mathrm{dd}$, $J=13.4,1.2 \mathrm{~Hz}, 1 \mathrm{H}), 3.29(\mathrm{dd}, J=13.4,1.2 \mathrm{~Hz}, 1 \mathrm{H}), 3.04-2.96(\mathrm{~m}, 2 \mathrm{H}), 2.19(\mathrm{~s}$,

\footnotetext{
${ }^{1}$ Cheng, Q.; Zhang, H.-J.; Yue, W.-J.; You, S.-L. Chem. 2017, 3, 428-436.
} 
$3 \mathrm{H}) ;{ }^{13} \mathrm{C} \mathrm{NMR}\left(126 \mathrm{MHz}, \mathrm{CDCl}_{3}\right) \delta 145.5,143.7,137.4,136.3,128.8,128.4,128.3$, 128.2, 126.8, 125.3, 113.6, 107.2, 74.9, 71.7, 67.5, 42.8; IR (Neat Film NaCl) 3468, 2953, 2928, 2857, 1617, 1465, 1378, 1255, 1154, 1086, 991, 921, $903 \mathrm{~cm}^{-1}$; HRMS (MM: ESI-APCI+) m/z calc'd for $\mathrm{C}_{20} \mathrm{H}_{23} \mathrm{INO}[\mathrm{M}+\mathrm{H}]^{+}:$: 420.0830 ; found: 420.0844 .

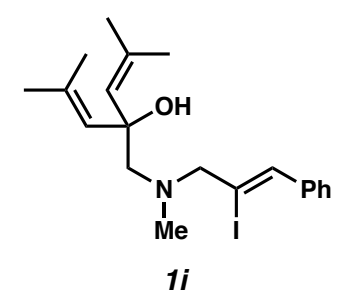

1i (214 mg, $0.502 \mathrm{mmol}$ ) was synthesized from sarconic methyl ester hydrochloride (350 mg, $2.51 \mathrm{mmol}) ; 20 \%$ yield ( 2 steps); $\mathrm{R}_{f}=0.38$ (1:8 EtOAc:hexanes); ${ }^{1} \mathrm{H}$ NMR $\left(400 \mathrm{MHz}, \mathrm{CDCl}_{3}\right) \delta 7.55-7.50(\mathrm{~m}, 2 \mathrm{H}), 7.35(\mathrm{tdd}, J=8.7,7.4,3.7 \mathrm{~Hz}, 3 \mathrm{H}), 6.96(\mathrm{~s}$, $1 \mathrm{H}), 5.40(\mathrm{dt}, J=2.7,1.3 \mathrm{~Hz}, 2 \mathrm{H}), 3.47(\mathrm{~s}, 2 \mathrm{H}), 2.71(\mathrm{~s}, 2 \mathrm{H}), 2.35(\mathrm{~s}, 3 \mathrm{H}), 1.76(\mathrm{~d}, J=$ $1.3 \mathrm{~Hz}, 6 \mathrm{H}), 1.71(\mathrm{~d}, J=1.4 \mathrm{~Hz}, 6 \mathrm{H}) ;{ }^{13} \mathrm{C} \mathrm{NMR}\left(101 \mathrm{MHz}, \mathrm{CDCl}_{3}\right) \delta 137.5,135.9$, $133.7,131.8,131.1,128.9,128.2,107.7,73.5,72.4,68.0,43.1,26.8,19.8$; IR (Neat Film NaCl) 2913, 2790, 1446, 1374, 1083, 1030, 750, $695 \mathrm{~cm}^{-1}$; HRMS (MM: ESIAPCI + ) m/z calc'd for $\mathrm{C}_{20} \mathrm{H}_{29} \mathrm{ONI}[\mathrm{M}+\mathrm{H}]^{+}:$426.1288; found: 426.1290 .

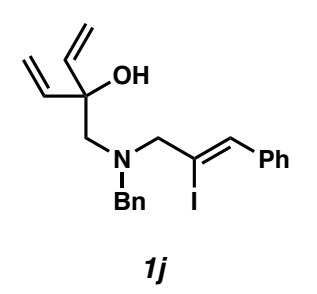

$\mathbf{1 j}$ (67.7 mg, $0.152 \mathrm{mmol}$ ) was synthesized from methyl 2-(benzylamino)acetate (75.8 mg, $0.423 \mathrm{mmol}) ; 36 \%$ yield (2 steps); $\mathrm{R}_{f}=0.38$ (1:8 EtOAc:hexanes); ${ }^{1} \mathrm{H}$ NMR (400 $\left.\mathrm{MHz}, \mathrm{CDCl}_{3}\right) \delta 7.53-7.44(\mathrm{~m}, 2 \mathrm{H}), 7.39-7.29(\mathrm{~m}, 8 \mathrm{H}), 6.95(\mathrm{~s}, 1 \mathrm{H}), 5.90(\mathrm{dd}, J=$ 17.2, $10.6 \mathrm{~Hz}, 2 \mathrm{H}), 5.40(\mathrm{dd}, J=17.2,1.5 \mathrm{~Hz}, 2 \mathrm{H}), 5.13(\mathrm{dd}, J=10.6,1.5 \mathrm{~Hz}, 2 \mathrm{H})$, 
$3.82(\mathrm{~s}, 1 \mathrm{H}), 3.78(\mathrm{~s}, 2 \mathrm{H}), 3.56(\mathrm{~s}, 2 \mathrm{H}), 2.80(\mathrm{~s}, 2 \mathrm{H}) ;{ }^{13} \mathrm{C} \mathrm{NMR}\left(101 \mathrm{MHz}, \mathrm{CDCl}_{3}\right) \delta$ $142.1,137.9,137.6,137.1,129.7,128.8,128.5,128.3,127.6,114.1,106.8,74.4,67.4$, 62.0, 58.8, 29.9; IR (Neat Film NaCl) 2916, 2849, 1250, 869, $696 \mathrm{~cm}^{-1}$; HRMS (MM: ESI-APCI+) m/z calc'd for $\mathrm{C}_{22} \mathrm{H}_{25} \mathrm{ONI}[\mathrm{M}+\mathrm{H}]^{+}:$:446.0975; found: 446.0984.

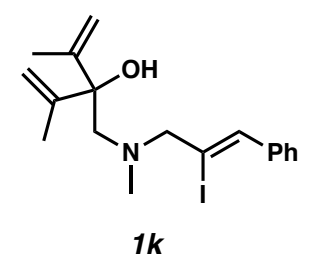

$1 \mathbf{k}$ (200 mg, $0.504 \mathrm{mmol}$ ) was synthesized from sarconic methyl ester hydrochloride (306 mg, $2.19 \mathrm{mmol}) ; 23 \%$ yield (2 steps); $\mathrm{R}_{f}=0.34$ (1:8 EtOAc:hexanes); ${ }^{1} \mathrm{H}$ NMR $\left(400 \mathrm{MHz}, \mathrm{CDCl}_{3}\right) \delta 7.55-7.48(\mathrm{~m}, 2 \mathrm{H}), 7.41-7.27(\mathrm{~m}, 3 \mathrm{H}), 6.96(\mathrm{~s}, 1 \mathrm{H}), 5.12(\mathrm{~s}$, 2H), $4.97(\mathrm{~s}, 2 \mathrm{H}), 4.63(\mathrm{~s}, 1 \mathrm{H}), 3.42(\mathrm{~s}, 2 \mathrm{H}), 2.96(\mathrm{~s}, 2 \mathrm{H}), 2.28(\mathrm{~s}, 3 \mathrm{H}), 1.71(\mathrm{~s}, 6 \mathrm{H}) ;{ }^{13} \mathrm{C}$ NMR (101 MHz, $\left.\mathrm{CDCl}_{3}\right) \delta 147.3,137.4,136.2,128.8,128.3,111.8,107.5,71.8,61.8$, 42.5, 18.9; IR (Neat Film NaCl) 2953, 2849, 2792, 1640, 1446, 1369, 1309, 1067, 1017 , 898, 750, $695 \mathrm{~cm}^{-1}$; HRMS (MM: ESI-APCI+) m/z calc'd for $\mathrm{C}_{18} \mathrm{H}_{25} \mathrm{ONI}[\mathrm{M}+\mathrm{H}]^{+}$: 398.0975; found: 398.0981.
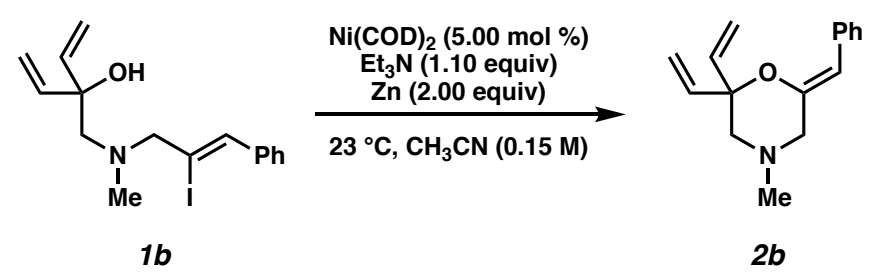

$\mathrm{Ni}$-catalyzed $\mathrm{C}-\mathrm{O}$ bond formation experiments were performed in a nitrogen-filled glove box. To a solution of aminoalcohol $1 \mathbf{b}(46.3 \mathrm{mg}, 0.125 \mathrm{mmol}, 1.00$ equiv) in $\operatorname{MeCN}(0.830 \mathrm{~mL})$ in a scintillation vial were added $\mathrm{Et}_{3} \mathrm{~N}(19 \mu \mathrm{L}, 0.138 \mathrm{mmol}, 1.10$ equiv), $\mathrm{Zn}$ powder (16.3 $\mathrm{mg}, 0.250 \mathrm{mmol}, 2.00$ equiv), and $\mathrm{Ni}(\mathrm{COD})_{2}(1.70 \mathrm{mg}$, $0.00627 \mathrm{mmol}, 0.05$ equiv). The reaction mixture was stirred at $23{ }^{\circ} \mathrm{C}$ for $24 \mathrm{~h}$. After 
the reaction was completed, the vial was removed from the glove box and uncapped. Solids were removed via filtration through a celite plug, and the resulting solution was concentrated under reduced pressure. The residue was purified by flash column chromatography (1:4 EtOAc:hexanes) to give morpholine $\mathbf{2 b}(22.3 \mathrm{mg}, 74 \%$ yield). $\mathrm{R}_{f}=0.35\left(1: 4\right.$ EtOAc:hexanes); ${ }^{1} \mathrm{H}$ NMR $\left(400 \mathrm{MHz}, \mathrm{CD}_{2} \mathrm{Cl}_{2}\right) \delta 7.68-7.61(\mathrm{~m}, 2 \mathrm{H})$, $7.28(\mathrm{dd}, J=8.4,7.1 \mathrm{~Hz}, 2 \mathrm{H}), 7.16-7.11(\mathrm{~m}, 1 \mathrm{H}), 5.93(\mathrm{dd}, J=17.4,10.9 \mathrm{~Hz}, 2 \mathrm{H})$, $5.47(\mathrm{~s}, 1 \mathrm{H}), 5.31(\mathrm{dd}, J=17.4,1.2 \mathrm{~Hz}, 2 \mathrm{H}), 5.17(\mathrm{dd}, J=10.9,1.2 \mathrm{~Hz}, 2 \mathrm{H}), 3.05-$ $2.98(\mathrm{~s}, 2 \mathrm{H}), 2.58(\mathrm{~s}, 2 \mathrm{H}), 2.26(\mathrm{~s}, 3 \mathrm{H}) ;{ }^{13} \mathrm{C} \mathrm{NMR}\left(101 \mathrm{MHz}, \mathrm{CD}_{2} \mathrm{Cl}_{2}\right) \delta 149.4,139.8$ 136.5, 128.9, 128.7, 126.3, 115.4, 108.0, 81.5, 62.8, 58.4, 46.3; IR (Neat Film NaCl) 2941, 2782, 1663, 1449, 1360, 1345, 1178, 1142, 983, 925, 754, $695 \mathrm{~cm}^{-1}$; HRMS (MM: ESI-APCI+) m/z calc'd for $\mathrm{C}_{16} \mathrm{H}_{20} \mathrm{ON}[\mathrm{M}+\mathrm{H}]^{+}:$242.1539; found: 242.1564 .

Representative procedure for the one-pot cycloetherification and Claisen rearrangement of tertiary alcohols
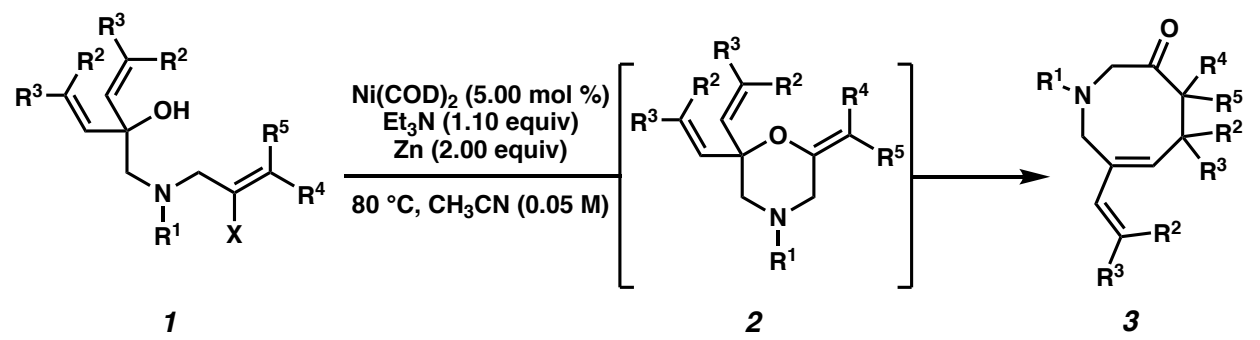

3

Experiments were performed in a nitrogen-filled glove box. To a solution of aminoalcohol 1 (0.0934 mmol, 1.00 equiv) in $\mathrm{MeCN}(1.90 \mathrm{~mL})$ in a scintillation vial were added $\mathrm{Et}_{3} \mathrm{~N}$ (14.3 $\mu \mathrm{L}, 0.103 \mathrm{mmol}, 1.10$ equiv), $\mathrm{Zn}$ powder (12.2 mg, $0.187 \mathrm{mmol}$, 2.00 equiv), and $\mathrm{Ni}(\mathrm{COD})_{2}(0.00467 \mathrm{mmol}, 0.05$ equiv $)$. The reaction mixture was stirred at $80^{\circ} \mathrm{C}$ for $24 \mathrm{~h}$. After the reaction was completed, the vial was removed from the glove box and uncapped. Solids were removed via filtration through a celite plug, 
and the resulting solution was concentrated under reduced pressure. The residue was purified by flash column chromatography to give hexahydroazocine 3 .

Note: Due to the COVID-19 pandemic, we are unable to conduct this experiment on a $1 \mathrm{mmol}$ scale due to limitations in our experimental lab in terms of capacity and personnel.

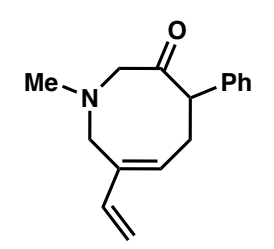

$3 b$

3b (11.0 mg, $0.0454 \mathrm{mmol})$ was synthesized from 1b (20.0 mg, $0.0541 \mathrm{mmol}) ; 84 \%$ yield; $\mathrm{R}_{f}=0.55$ (1:2 EtOAc:hexanes); ${ }^{1} \mathrm{H} \mathrm{NMR}\left(400 \mathrm{MHz}, \mathrm{CDCl}_{3}\right) \delta 7.46-7.39(\mathrm{~m}$, 2H), $7.38-7.32(\mathrm{~m}, 2 \mathrm{H}), 7.31-7.26(\mathrm{~m}, 1 \mathrm{H}), 6.30(\mathrm{ddd}, J=17.7,11.0,0.8 \mathrm{~Hz}, 1 \mathrm{H})$, $6.00-5.89(\mathrm{~m}, 1 \mathrm{H}), 5.16(\mathrm{~d}, J=17.7 \mathrm{~Hz}, 1 \mathrm{H}), 5.01(\mathrm{dt}, J=11.0,0.7 \mathrm{~Hz}, 1 \mathrm{H}), 3.97-$ $3.82(\mathrm{~m}, 2 \mathrm{H}), 3.63-3.52(\mathrm{~m}, 1 \mathrm{H}), 3.44-3.29(\mathrm{~m}, 2 \mathrm{H}), 2.93-2.81(\mathrm{~m}, 1 \mathrm{H}), 2.54$ (ddd, $J=9.9,7.9,4.3 \mathrm{~Hz}, 1 \mathrm{H}), 2.44(\mathrm{~s}, 3 \mathrm{H}) ;{ }^{13} \mathrm{C} \mathrm{NMR}\left(101 \mathrm{MHz}, \mathrm{CDCl}_{3}\right) \delta 210.0,139.6$, $138.1,136.8,131.8,128.9,128.0,127.6,112.3,65.4,60.8,56.2,46.0,29.2$; IR (Neat Film NaCl) 2939, 1702, 1452, 902, 765, $701 \mathrm{~cm}^{-1}$; HRMS (MM: ESI-APCI+) m/z calc'd for $\mathrm{C}_{16} \mathrm{H}_{20} \mathrm{ON}[\mathrm{M}+\mathrm{H}]^{+}:$242.1539; found: 242.1548 .

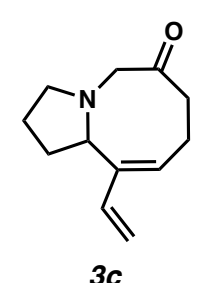

3c (4.20 mg, $0.0219 \mathrm{mmol})$ was synthesized from 1c (10.6 mg, $0.0332 \mathrm{mmol}) ; 66 \%$ yield; $\mathrm{R}_{f}=0.35$ (1:8 EtOAc:hexanes); ${ }^{1} \mathrm{H}$ NMR (400 MHz, $\left.\mathrm{CD}_{2} \mathrm{Cl}_{2}\right) \delta 6.19(\mathrm{dd}, J=$ 17.7, 11.1 Hz, 1H), 5.74 (t, $J=8.9 \mathrm{~Hz}, 1 \mathrm{H}), 5.07$ (d, $J=17.7 \mathrm{~Hz}, 1 \mathrm{H}), 4.93$ (d, $J=11.2$ $\mathrm{Hz}, 1 \mathrm{H}), 4.15-4.01(\mathrm{~m}, 1 \mathrm{H}), 3.59(\mathrm{~d}, J=16.1 \mathrm{~Hz}, 1 \mathrm{H}), 3.19(\mathrm{td}, J=8.4,2.0 \mathrm{~Hz}, 1 \mathrm{H})$, $3.05(\mathrm{dd}, J=9.7,7.6 \mathrm{~Hz}, 1 \mathrm{H}), 2.89(\mathrm{ddd}, J=14.0,7.7,1.7 \mathrm{~Hz}, 1 \mathrm{H}), 2.82(\mathrm{dd}, J=16.1$, 
$1.9 \mathrm{~Hz}, 1 \mathrm{H}), 2.39$ (dddd, $J=13.9,11.8,7.6,2.0 \mathrm{~Hz}, 1 \mathrm{H}), 2.30-2.19$ (m, 2H), 1.99 (dddd, $J=12.2,8.8,7.6,1.6 \mathrm{~Hz}, 1 \mathrm{H}), 1.87(\mathrm{~m}, J=12.8,11.2,9.5,8.0,5.0 \mathrm{~Hz}, 1 \mathrm{H})$, $1.81-1.70(\mathrm{~m}, 1 \mathrm{H}), 1.64(\mathrm{dddd}, J=12.5,11.2,9.7,5.7 \mathrm{~Hz}, 1 \mathrm{H}) ;{ }^{13} \mathrm{C} \mathrm{NMR}(101 \mathrm{MHz}$, $\left.\mathrm{CD}_{2} \mathrm{Cl}_{2}\right) \delta 212.5,140.6,139.8,131.7,111.9,67.4,67.4,56.1,53.5,43.4,33.4,23.2$, 22.7; IR (Neat Film NaCl) 2945, 2798, 1706, 1222, $895 \mathrm{~cm}^{-1}$; HRMS (MM: ESI-APCI+) m/z calc'd for $\mathrm{C}_{12} \mathrm{H}_{18} \mathrm{ON}[\mathrm{M}+\mathrm{H}]^{+}:$192.1383; found: 192.1383 .

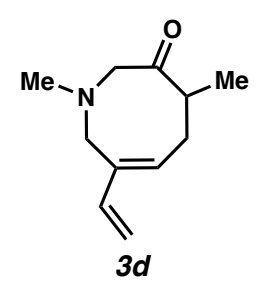

3d (7.30 mg, $0.0410 \mathrm{mmol})$ was synthesized from 1d (20.0 mg, $0.0651 \mathrm{mmol}) ; 63 \%$ yield; $\mathrm{R}_{f}=0.25$ (1:4 EtOAc:hexanes); ${ }^{1} \mathrm{H}$ NMR (400 MHz, $\left.\mathrm{CDCl}_{3}\right) \delta 6.27(\mathrm{ddd}, J=$ $17.5,10.9,0.8 \mathrm{~Hz}, 1 \mathrm{H}), 5.91(\mathrm{t}, J=8.5 \mathrm{~Hz}, 1 \mathrm{H}), 5.18(\mathrm{dt}, J=17.6,0.7 \mathrm{~Hz}, 1 \mathrm{H}), 4.99$ (dt, $J=10.8,0.8 \mathrm{~Hz}, 1 \mathrm{H}), 3.42-3.26(\mathrm{~m}, 2 \mathrm{H}), 3.17(\mathrm{~d}, J=2.1 \mathrm{~Hz}, 2 \mathrm{H}), 2.78-2.67(\mathrm{~m}$, 1H), $2.67-2.53(\mathrm{~m}, 2 \mathrm{H}), 2.46(\mathrm{~s}, 3 \mathrm{H}), 1.14(\mathrm{~d}, J=6.7 \mathrm{~Hz}, 3 \mathrm{H}) ;{ }^{13} \mathrm{C}$ NMR (101 MHz, $\left.\mathrm{CDCl}_{3}\right) \delta 213.7,139.5,137.2,132.2,112.4,64.0,54.0,48.8,45.4,31.1,16.4$; IR (Neat Film NaCl) 2969, 2931, 1702, 1451, $898 \mathrm{~cm}^{-1}$; HRMS (MM: ESI-APCI+) m/z calc'd for $\mathrm{C}_{11} \mathrm{H}_{18} \mathrm{ON}[\mathrm{M}+\mathrm{H}]^{+}:$180.1383; found: 180.1386 .

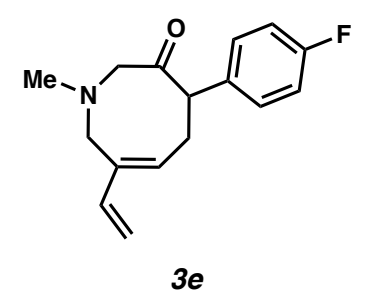

3e (9.50 mg, $0.0368 \mathrm{mmol})$ was synthesized from 1d (25.0 mg, $0.0646 \mathrm{mmol}) ; 57 \%$ yield; $\mathrm{R}_{f}=0.27$ (1:4 EtOAc:hexanes); ${ }^{1} \mathrm{H}$ NMR (500 MHz, $\left.\mathrm{CDCl}_{3}\right) \delta 7.41-7.35(\mathrm{~m}$, 2H), $7.07-7.01(\mathrm{~m}, 2 \mathrm{H}), 6.28(\mathrm{ddd}, J=17.7,11.0,0.8 \mathrm{~Hz}, 1 \mathrm{H}), 5.99-5.88(\mathrm{~m}, 1 \mathrm{H})$, 
$5.16(\mathrm{~d}, J=17.6 \mathrm{~Hz}, 1 \mathrm{H}), 5.01(\mathrm{dt}, J=11.0,0.7 \mathrm{~Hz}, 1 \mathrm{H}), 3.91-3.79(\mathrm{~m}, 2 \mathrm{H}), 3.54(\mathrm{dt}$, $J=15.1,1.2 \mathrm{~Hz}, 1 \mathrm{H}), 3.35-3.27(\mathrm{~m}, 2 \mathrm{H}), 2.89(\mathrm{dt}, J=15.6,0.7 \mathrm{~Hz}, 1 \mathrm{H}), 2.59-2.49$ (m, 1H), $2.43(\mathrm{~s}, 3 \mathrm{H}) ;{ }^{13} \mathrm{C} \mathrm{NMR}\left(126 \mathrm{MHz}, \mathrm{CDCl}_{3}\right) \delta 210.1,162.2(\mathrm{~d}, J=246.2 \mathrm{~Hz})$, 139.5, 136.9, 134.0 (d, $J=3.3 \mathrm{~Hz}), 131.4,129.7$ (d, $J=8.2 \mathrm{~Hz}), 115.7(\mathrm{~d}, J=21.1 \mathrm{~Hz})$, $112.4,65.6,59.9,56.5,46.2,29.5 ;{ }^{19} \mathrm{~F}$ NMR $\left(282 \mathrm{MHz}, \mathrm{CDCl}_{3}\right) \delta$-115.2; IR (Neat Film NaCl) 2929, 1702, 1508, 1224, 1160, $832 \mathrm{~cm}^{-1}$; HRMS (MM: ESI-APCI+) m/z calc'd for $\mathrm{C}_{16} \mathrm{H}_{19} \mathrm{ONF}[\mathrm{M}+\mathrm{H}]^{+}:$260.1445; found: 260.1449 .

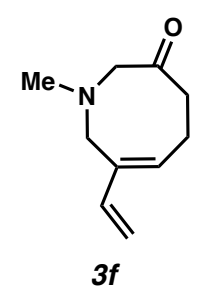

3f $(5.30 \mathrm{mg}, 0.0321 \mathrm{mmol})$ was synthesized from 1 f $(20.0 \mathrm{mg}, 0.0682 \mathrm{mmol}) ; 47 \%$ yield; $\mathrm{R}_{f}=0.30$ (1:2 EtOAc:hexanes); ${ }^{1} \mathrm{H}$ NMR $\left(500 \mathrm{MHz}, \mathrm{CDCl}_{3}\right) \delta 6.28(\mathrm{ddd}, J=$ 17.6, 11.0, $0.8 \mathrm{~Hz}, 1 \mathrm{H}), 5.92(\mathrm{tq}, J=8.5,0.8 \mathrm{~Hz}, 1 \mathrm{H}), 5.18(\mathrm{dt}, J=17.5,0.7 \mathrm{~Hz}, 1 \mathrm{H})$, $5.02-4.97(\mathrm{~m}, 1 \mathrm{H}), 3.36(\mathrm{~s}, 2 \mathrm{H}), 3.16(\mathrm{~s}, 2 \mathrm{H}), 2.72(\mathrm{dt}, J=8.6,6.9 \mathrm{~Hz}, 2 \mathrm{H}), 2.58(\mathrm{dd}$, $J=7.8,5.8 \mathrm{~Hz}, 2 \mathrm{H}), 2.46(\mathrm{~s}, 3 \mathrm{H}) ;{ }^{13} \mathrm{C} \mathrm{NMR}\left(126 \mathrm{MHz}, \mathrm{CDCl}_{3}\right) \delta 211.9,139.4,137.0$, 133.0, 112.4, 65.3, 54.2, 45.3, 44.5, 22.8; IR (Neat Film NaCl) 2924, 2851, 1703, 1560 , 1450, 1042, $902 \mathrm{~cm}^{-1}$; HRMS (MM: ESI-APCI+) m/z calc'd for $\mathrm{C}_{10} \mathrm{H}_{16} \mathrm{ON}[\mathrm{M}+\mathrm{H}]^{+}$: 166.1226; found: 166.1228 .

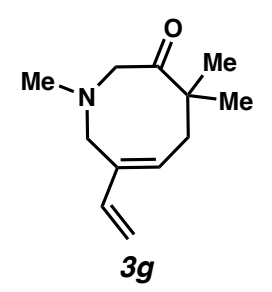


$\mathbf{3 g}$ (13.5 mg, $0.0700 \mathrm{mmol})$ was synthesized from $1 \mathrm{~g}$ (30.0 mg, $0.109 \mathrm{mmol})$; 64\% yield; $\mathrm{R}_{f}=0.35$ (1:4 EtOAc:hexanes); ${ }^{1} \mathrm{H}$ NMR $\left(400 \mathrm{MHz}, \mathrm{CDCl}_{3}\right) \delta 6.23$ (ddd, $J=17.6,11.0$, $0.8 \mathrm{~Hz}, 1 \mathrm{H}), 5.85(\mathrm{tq}, J=8.8,0.8 \mathrm{~Hz}, 1 \mathrm{H}), 5.13(\mathrm{~d}, J=17.6 \mathrm{~Hz}, 1 \mathrm{H}), 4.95(\mathrm{dd}, J=11.0$, $0.8 \mathrm{~Hz}, 1 \mathrm{H}), 3.38(\mathrm{~s}, 2 \mathrm{H}), 3.23(\mathrm{~s}, 2 \mathrm{H}), 2.78-2.64(\mathrm{~m}, 2 \mathrm{H}), 2.42(\mathrm{~s}, 3 \mathrm{H}), 1.17(\mathrm{~s}, 6 \mathrm{H})$; ${ }^{13} \mathrm{C} \mathrm{NMR}\left(101 \mathrm{MHz}, \mathrm{CDCl}_{3}\right) \delta 214.2,139.8,137.4,131.9,112.0,64.3,55.4,50.7,45.4$, 37.5, 24.9; IR (Neat Film NaCl) 2970, 2930, 1702, 1452, 1126, $891 \mathrm{~cm}^{-1}$; HRMS (MM: ESI-APCI+) m/z calc'd for $\mathrm{C}_{12} \mathrm{H}_{20} \mathrm{ON}[\mathrm{M}+\mathrm{H}]^{+}:$194.1539; found: 194.1547.

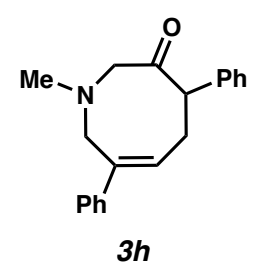

3h (49.1 mg, 0.169 mmol) was synthesized from $\mathbf{1 h}$ (102 mg, 0.242 mmol); 70\% yield; $\mathrm{R}_{f}=0.53$ (1:4 EtOAc:hexanes); ${ }^{1} \mathrm{H} \mathrm{NMR}\left(400 \mathrm{MHz}, \mathrm{CDCl}_{3}\right) \delta{ }^{1} \mathrm{H}$ NMR $(400 \mathrm{MHz}$, $\left.\mathrm{CDCl}_{3}\right) \delta 7.42-7.14(\mathrm{~m}, 10 \mathrm{H}), 6.12-6.03(\mathrm{~m}, 1 \mathrm{H}), 3.94-3.78(\mathrm{~m}, 3 \mathrm{H}), 3.34(\mathrm{dd}, J=$ 15.2, $1.3 \mathrm{~Hz}, 2 \mathrm{H}), 2.93(\mathrm{~d}, J=15.2 \mathrm{~Hz}, 1 \mathrm{H}), 2.63-2.51(\mathrm{~m}, 1 \mathrm{H}), 2.37(\mathrm{~s}, 3 \mathrm{H}) ;{ }^{13} \mathrm{C}$ NMR (101 MHz, $\left.\mathrm{CDCl}_{3}\right) \delta 210.1,142.9,139.3,138.0,128.7,128.5,128.2,127.9,127.3$, 127.1, 126.2, 65.0, 60.8, 60.7, 45.9, 29.0; IR (Neat Film NaCl) 3058, 3029, 2900, 2928, $2825,2801,1956,1882,1806,1698,1597,1490,1442,1276,1260,1124,876,761$, $702 \mathrm{~cm}^{-1}$; HRMS (MM: ESI-APCI+) m/z calc'd for $\mathrm{C}_{20} \mathrm{H}_{22} \mathrm{NO}[\mathrm{M}+\mathrm{H}]^{+}:$292.1623; found: 292.2742 .

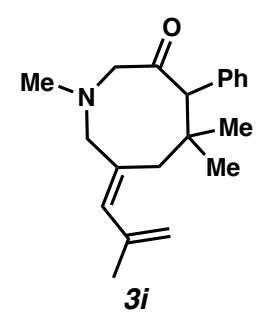


3i (5.00 mg, $0.0169 \mathrm{mmol})$ was synthesized from 1i (30.0 mg, $0.0705 \mathrm{mmol}) ; 24 \%$ yield; $\mathrm{R}_{f}=0.45$ (1:8 EtOAc:hexanes); ${ }^{1} \mathrm{H}$ NMR $\left(400 \mathrm{MHz}, \mathrm{CDCl}_{3}\right) \delta 7.55-7.47(\mathrm{~m}$, 2H), $7.23(\mathrm{dd}, J=8.6,7.0 \mathrm{~Hz}, 3 \mathrm{H}), 5.89(\mathrm{~s}, 1 \mathrm{H}), 4.91(\mathrm{~d}, J=5.2 \mathrm{~Hz}, 2 \mathrm{H}), 4.65(\mathrm{~s}, 1 \mathrm{H})$, $3.44(\mathrm{~d}, J=12.7 \mathrm{~Hz}, 1 \mathrm{H}), 3.10(\mathrm{dd}, J=15.4,1.9 \mathrm{~Hz}, 1 \mathrm{H}), 3.02(\mathrm{~d}, J=13.1 \mathrm{~Hz}, 1 \mathrm{H})$, $2.72(\mathrm{~d}, J=3.8 \mathrm{~Hz}, 1 \mathrm{H}), 2.68(\mathrm{~d}, J=6.5 \mathrm{~Hz}, 1 \mathrm{H}), 2.45(\mathrm{~s}, 3 \mathrm{H}), 2.10(\mathrm{~d}, J=13.2 \mathrm{~Hz}$ 1H), $1.81(\mathrm{~s}, 3 \mathrm{H}), 1.04$ (s, 3H), $0.74(\mathrm{~s}, 3 \mathrm{H}) ;{ }^{13} \mathrm{C} \mathrm{NMR}\left(101 \mathrm{MHz}, \mathrm{CDCl}_{3}\right) \delta$ 212.3, $143.0,138.1,134.8,134.5,131.0,127.6,126.7,114.6,71.6,70.7,59.2,46.5,42.7,41.3$, 31.5, 23.1, 21.8; IR (Neat Film NaCl) 2943, 2796, 1709, 1450, 1357, 1127, $704 \mathrm{~cm}^{-1}$; HRMS (MM: ESI-APCI+) m/z calc'd for $\mathrm{C}_{20} \mathrm{H}_{28} \mathrm{ON}[\mathrm{M}+\mathrm{H}]^{+}$: 298.2165; found: 298.2168 .

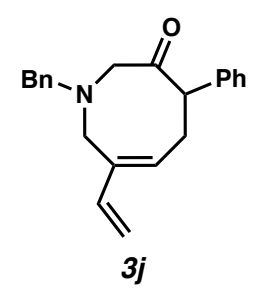

3j (22.0 mg, $0.0691 \mathrm{mmol}$ ) was synthesized from 1j (41.6 mg, $0.0934 \mathrm{mmol}) ; 74 \%$ yield; $\mathrm{R}_{f}=0.35$ (1:8 EtOAc:hexanes); ${ }^{1} \mathrm{H}$ NMR (400 MHz, $\left.\mathrm{CDCl}_{3}\right) \delta 7.43-7.31(\mathrm{~m}$, 4H), $7.33-7.20(\mathrm{~m}, 6 \mathrm{H}), 6.27(\mathrm{dd}, J=17.6,11.0 \mathrm{~Hz}, 1 \mathrm{H}), 5.97(\mathrm{t}, J=8.4 \mathrm{~Hz}, 1 \mathrm{H})$, $5.05(\mathrm{~d}, J=17.6 \mathrm{~Hz}, 1 \mathrm{H}), 4.96(\mathrm{~d}, J=11.0 \mathrm{~Hz}, 1 \mathrm{H}), 3.96(\mathrm{td}, J=11.5,8.8 \mathrm{~Hz}, 1 \mathrm{H})$, $3.90-3.83(\mathrm{~m}, 1 \mathrm{H}), 3.69(\mathrm{~d}, J=1.7 \mathrm{~Hz}, 2 \mathrm{H}), 3.59(\mathrm{~d}, J=15.2 \mathrm{~Hz}, 1 \mathrm{H}), 3.53-3.43(\mathrm{~m}$, $1 \mathrm{H}), 3.34(\mathrm{~d}, J=15.2 \mathrm{~Hz}, 1 \mathrm{H}), 2.97(\mathrm{~d}, J=15.2 \mathrm{~Hz}, 1 \mathrm{H}), 2.66(\mathrm{ddd}, J=11.7,8.2,5.2$ $\mathrm{Hz}, 1 \mathrm{H}) ;{ }^{13} \mathrm{C} \mathrm{NMR}\left(101 \mathrm{MHz}, \mathrm{CDCl}_{3}\right) \delta 210.6,139.5,138.4,138.3,136.8,131.4,129.5$, 128.8, 128.6, 127.9, 127.6, 127.4, 112.4, 63.9, 62.6, 60.1, 54.8, 29.3; IR (Neat Film $\mathrm{NaCl})$ 2923, 1703, 1494, 1453, 901, $699 \mathrm{~cm}^{-1}$; HRMS (MM: ESI-APCI+) m/z calc'd for $\mathrm{C}_{22} \mathrm{H}_{24} \mathrm{ON}[\mathrm{M}+\mathrm{H}]^{+}: 318.1852$; found: 318.1858 . 


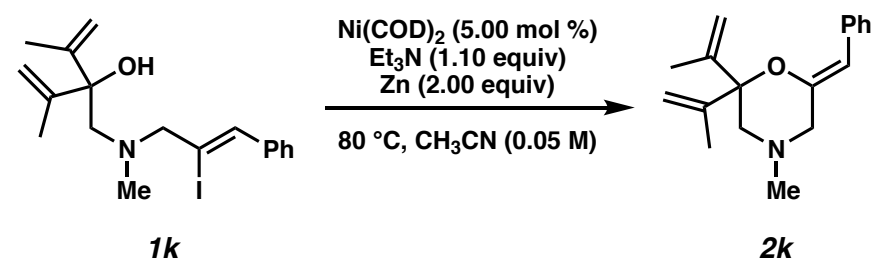

Experiments were performed in a nitrogen-filled glove box. To a solution of aminoalcohol $\mathbf{1 k}(50.0 \mathrm{mg}, 0.126 \mathrm{mmol}, 1.00$ equiv) in $\mathrm{MeCN}(2.52 \mathrm{~mL})$ in a scintillation vial were added $\mathrm{Et}_{3} \mathrm{~N}$ (19.3 $\mu \mathrm{L}, 0.139 \mathrm{mmol}, 1.10$ equiv), Zn powder (16.5 $\mathrm{mg}, 0.252 \mathrm{mmol}, 2.00$ equiv), and $\mathrm{Ni}(\mathrm{COD})_{2}(0.00629 \mathrm{mmol}, 0.05$ equiv). The reaction mixture was stirred at $80{ }^{\circ} \mathrm{C}$ for $24 \mathrm{~h}$. After the reaction was completed, the vial was removed from the glove box and uncapped. Solids were removed via filtration through a celite plug, and the resulting solution was concentrated under reduced pressure. The residue was purified by flash column chromatography to give morpholine $\mathbf{2 k}$ (34.0 mg, $99 \%$ yield).

$\mathrm{R}_{f}=0.25\left(1: 8\right.$ EtOAc:hexanes); ${ }^{1} \mathrm{H} \mathrm{NMR}\left(400 \mathrm{MHz}, \mathrm{CDCl}_{3}\right) \delta 7.67-7.58(\mathrm{~m}, 2 \mathrm{H})$, $7.28(\mathrm{dd}, J=8.5,7.1 \mathrm{~Hz}, 3 \mathrm{H}), 7.20-7.09(\mathrm{~m}, 1 \mathrm{H}), 5.47(\mathrm{~s}, 1 \mathrm{H}), 5.04(\mathrm{~s}, 2 \mathrm{H}), 5.01(\mathrm{p}$, $J=1.4 \mathrm{~Hz}, 3 \mathrm{H}), 3.09(\mathrm{~s}, 2 \mathrm{H}), 2.79(\mathrm{~s}, 2 \mathrm{H}), 2.31(\mathrm{~s}, 3 \mathrm{H}), 1.73(\mathrm{dd}, J=1.5,0.8 \mathrm{~Hz}, 6 \mathrm{H})$; ${ }^{13} \mathrm{C} \mathrm{NMR}\left(101 \mathrm{MHz}, \mathrm{CDCl}_{3}\right) \delta 148.4,144.1,135.9,128.7,128.2,125.8,113.2,107.5$, 85.4, 59.5, 58.4, 46.5, 19.2; IR (Neat Film NaCl) 2971, 2767, 1663, 1645, 1448, 1361, 1345, 1249, 1179, 1141, 1078, 1060, 984, 903, $695 \mathrm{~cm}^{-1}$; HRMS (MM: ESI-APCI+) $\mathrm{m} / \mathrm{z}$ calc'd for $\mathrm{C}_{18} \mathrm{H}_{24} \mathrm{ON}[\mathrm{M}+\mathrm{H}]^{+}:$270.1852; found: 270.1862 .

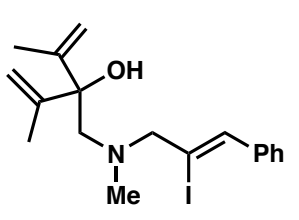

$1 k$

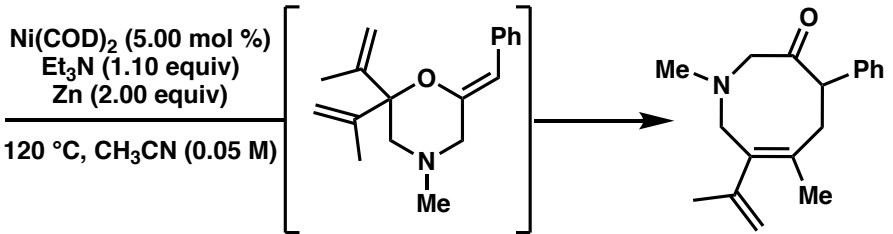

$2 k$
$3 k$ 
Experiments were performed in a nitrogen-filled glove box. To a solution of aminoalcohol $1 \mathbf{k}(20.0 \mathrm{mg}, 0.0503 \mathrm{mmol}, 1.00$ equiv) in $\mathrm{MeCN}(1.00 \mathrm{~mL})$ in a scintillation vial were added $\mathrm{Et}_{3} \mathrm{~N}(7.70 \mu \mathrm{L}, 0.0553 \mathrm{mmol}, 1.10$ equiv), Zn powder (6.60 mg, $0.101 \mathrm{mmol}, 2.00$ equiv), and $\mathrm{Ni}(\mathrm{COD})_{2}(0.70 \mathrm{mg}, 0.00252 \mathrm{mmol}, 0.05$ equiv). The reaction mixture was stirred at $80^{\circ} \mathrm{C}$ for $24 \mathrm{~h}$. After the reaction was completed, the vial was removed from the glove box and uncapped. Solids were removed via filtration through a celite plug, and the resulting solution was concentrated under reduced pressure. The residue was purified by flash column chromatography to give hexahydroazocine $3 \mathbf{k}(6.60 \mathrm{mg}, 49 \%$ yield $)$.

$\mathrm{R}_{f}=0.27\left(1: 8\right.$ EtOAc:hexanes); ${ }^{1} \mathrm{H} \mathrm{NMR}\left(500 \mathrm{MHz}, \mathrm{CDCl}_{3}\right) \delta 7.53-7.46(\mathrm{~m}, 2 \mathrm{H})$, $7.39-7.31(\mathrm{~m}, 2 \mathrm{H}), 7.33-7.25(\mathrm{~m}, 1 \mathrm{H}), 4.91(\mathrm{dq}, J=3.0,1.6 \mathrm{~Hz}, 1 \mathrm{H}), 4.59(\mathrm{dq}, J=$ $1.9,0.9 \mathrm{~Hz}, 1 \mathrm{H}), 4.22(\mathrm{t}, J=12.2 \mathrm{~Hz}, 1 \mathrm{H}), 3.91(\mathrm{dd}, J=12.4,6.3 \mathrm{~Hz}, 1 \mathrm{H}), 3.68-3.59$ (m, 1H), $3.26(\mathrm{dd}, J=16.0,0.9 \mathrm{~Hz}, 1 \mathrm{H}), 2.82(\mathrm{dt}, J=16.0,1.0 \mathrm{~Hz}, 1 \mathrm{H}), 2.75(\mathrm{~d}, J=$ $14.8 \mathrm{~Hz}, 1 \mathrm{H}), 2.33(\mathrm{~s}, 3 \mathrm{H}), 2.12(\mathrm{dd}, J=12.0,6.3 \mathrm{~Hz}, 1 \mathrm{H}), 1.76(\mathrm{~m}, 6 \mathrm{H}) ;{ }^{13} \mathrm{C} \mathrm{NMR}$ $\left(126 \mathrm{MHz}, \mathrm{CDCl}_{3}\right) \delta 210.0,146.6,138.2,134.8,132.1,128.9,128.2,127.5,113.3,65.8$, 61.7, 60.3, 46.1, 34.8, 22.0, 21.0; IR (Neat Film NaCl) 2913, 2794, 1701, 1450, 903, 768, $699 \mathrm{~cm}^{-1}$; HRMS (MM: ESI-APCI+) m/z calc'd for $\mathrm{C}_{18} \mathrm{H}_{24} \mathrm{ON}[\mathrm{M}+\mathrm{H}]^{+}: 270.1852$; found: 270.1871 .

\section{Representative procedure for secondary aminoalcohols}
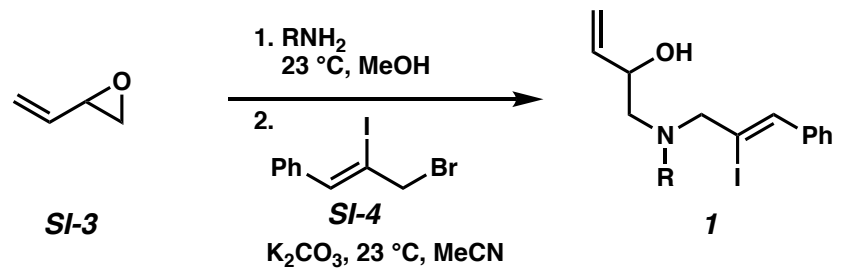
To epoxide SI-3 (4.28 mmol, 1.00 equiv) was added $\mathrm{RNH}_{2}$ (42.8 mmol, 10.0 equiv). The solution was stirred for $12 \mathrm{~h}$ at $23{ }^{\circ} \mathrm{C}$. Volatiles were evaporated, and the residue was used without further purification.

To a solution of the amine (4.28 mmol, 1.00 equiv) in $\mathrm{MeCN}(11.0 \mathrm{~mL})$ were added $\mathrm{K}_{2} \mathrm{CO}_{3}$ (21.4 mmol, 5.00 equiv) and allyl bromide SI-4 (2.14 mmol, 0.50 equiv). The solution was stirred for $12 \mathrm{~h}$ at $23{ }^{\circ} \mathrm{C}$. After the reaction was completed, water was added. The aqueous phase was extracted with EtOAc $(3 \times 7.00 \mathrm{~mL})$. The combined organic phases were washed with brine, dried over anhydrous $\mathrm{MgSO}_{4}$ and concentrated in vacuo. The residue was purified by flash column chromatography (1:4 EtOAc:hexanes) on silica gel to give secondary alcohols 1 in 14-36\% 2-step yield based on equivalent of allyl bromide SI-4.

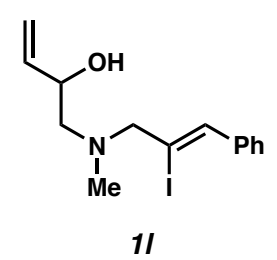

11 (515 mg, $1.50 \mathrm{mmol}$ ) was synthesized from 3,4-epoxy-1-butene (300 mg, 4.28 mmol); 35\% yield (2 steps); $\mathrm{R}_{f}=0.65$ (1:2 EtOAc:hexanes); ${ }^{1} \mathrm{H}$ NMR (400 MHz, $\left.\mathrm{CDCl}_{3}\right) \delta 7.60-7.55(\mathrm{~m}, 2 \mathrm{H}), 7.43-7.34(\mathrm{~m}, 3 \mathrm{H}), 7.10-6.98(\mathrm{~s}, \mathrm{br}, 1 \mathrm{H}), 5.84$ (ddd, $J=17.2,10.5,5.8 \mathrm{~Hz}, 1 \mathrm{H}), 5.45-5.37(\mathrm{~m}, 1 \mathrm{H}), 5.21(\mathrm{dt}, J=10.5,1.5 \mathrm{~Hz}, 1 \mathrm{H}), 4.29$ (s, br, 1H), $3.54(\mathrm{~s}, \mathrm{br}, 1 \mathrm{H}), 3.41(\mathrm{~s}, \mathrm{br}, 1 \mathrm{H}), 2.58(\mathrm{~s}, \mathrm{br}, 2 \mathrm{H}), 2.40(\mathrm{~s}, 3 \mathrm{H}) ;{ }^{13} \mathrm{C} \mathrm{NMR}$ $\left(101 \mathrm{MHz}, \mathrm{CDCl}_{3}\right) \delta 138.0,137.2,136.9,128.8,128.4,128.3,116.3,70.7,68.7,62.8$ 61.1, 41.2; IR (Neat Film NaCl) 3435, 2795, 1491, 1446, 1083, 1029, 921, 750, 695 $\mathrm{cm}^{-1}$; HRMS (MM: ESI-APCI+) m/z calc'd for $\mathrm{C}_{14} \mathrm{H}_{19} \mathrm{NOI}[\mathrm{M}+\mathrm{H}]^{+}:$344.0506; found: 344.0523. 


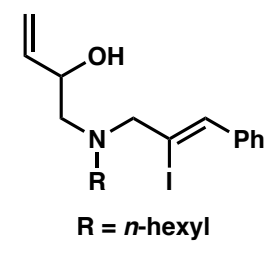

1n

1n (731 mg, $1.77 \mathrm{mmol}$ ) was synthesized from 3,4-epoxy-1-butene (452 mg, 6.45 mmol); 27\% yield (2 steps); $\mathrm{R}_{f}=0.69$ (1:4 EtOAc:hexanes); ${ }^{1} \mathrm{H}$ NMR (500 MHz, $\left.\mathrm{CDCl}_{3}\right) \delta 7.57-7.49(\mathrm{~m}, 2 \mathrm{H}), 7.41-7.29(\mathrm{~m}, 3 \mathrm{H}), 6.97(\mathrm{~s}, 1 \mathrm{H}), 5.81(\mathrm{ddd}, J=17.3$, 10.5, $5.7 \mathrm{~Hz}, 1 \mathrm{H}), 5.36(\mathrm{dt}, J=17.2,1.6 \mathrm{~Hz}, 1 \mathrm{H}), 5.17(\mathrm{dt}, J=10.5,1.5 \mathrm{~Hz}, 1 \mathrm{H}), 4.27$ $-4.13(\mathrm{~m}, 1 \mathrm{H}), 3.72(\mathrm{~s}, 1 \mathrm{H}), 3.59(\mathrm{~d}, J=13.9 \mathrm{~Hz}, 1 \mathrm{H}), 3.33(\mathrm{~d}, J=13.8 \mathrm{~Hz}, 1 \mathrm{H}), 2.64$ $(\mathrm{dt}, J=13.1,7.9 \mathrm{~Hz}, 1 \mathrm{H}), 2.60-2.41(\mathrm{~m}, 3 \mathrm{H}), 1.61-1.44(\mathrm{~m}, 2 \mathrm{H}), 1.41-1.21(\mathrm{~m}$, 6H), $0.96-0.83(\mathrm{~m}, 3 \mathrm{H}) ;{ }^{13} \mathrm{C} \mathrm{NMR}\left(126 \mathrm{MHz}, \mathrm{CDCl}_{3}\right) \delta 138.2,137.5,136.3,128.8$, $128.28,128.25,116.1,107.6,68.8,67.6,59.7,53.4,31.8,27.3,26.6,22.8,14.2$; IR (Neat Film NaCl) 3460, 2953, 2928, 2856, 1491, 1445, 1362, 1286, 1150, 1080, 992 , 921, 860, 749, $695 \mathrm{~cm}^{-1}$; HRMS (MM: ESI-APCI+) m/z calc'd for $\mathrm{C}_{19} \mathrm{H}_{29} \mathrm{INO}[\mathrm{M}+\mathrm{H}]^{+}$: 414.1288; found: 414.1296 .

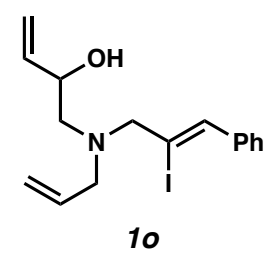

10 (552 mg, $1.49 \mathrm{mmol}$ ) was synthesized from 3,4-epoxy-1-butene (452 mg, 6.45 mmol); 23\% yield (2 steps); $\mathrm{R}_{f}=0.61$ (1:4 EtOAc:hexanes); ${ }^{1} \mathrm{H}$ NMR (300 MHz, $\left.\mathrm{CDCl}_{3}\right){ }^{1} \mathrm{H} \mathrm{NMR}\left(300 \mathrm{MHz}, \mathrm{CDCl}_{3}\right) \delta 7.58-7.49(\mathrm{~m}, 2 \mathrm{H}), 7.42-7.28(\mathrm{~m}, 3 \mathrm{H}), 6.98$ (s, $1 \mathrm{H}), 5.99-5.85(\mathrm{~m}, 1 \mathrm{H}), 5.86-5.70(\mathrm{~m}, 1 \mathrm{H}), 5.42-5.09(\mathrm{~m}, 4 \mathrm{H}), 4.33-4.16(\mathrm{~m}$, $1 \mathrm{H}), 3.76-3.55(\mathrm{~m}, 2 \mathrm{H}), 3.35(\mathrm{dd}, J=14.2,4.7 \mathrm{~Hz}, 2 \mathrm{H}), 3.15(\mathrm{dd}, J=14.3,7.7 \mathrm{~Hz}$, 1H), $2.57(\mathrm{qd}, J=12.8,6.8 \mathrm{~Hz}, 2 \mathrm{H}) ;{ }^{13} \mathrm{C} \mathrm{NMR}\left(126 \mathrm{MHz}, \mathrm{CDCl}_{3}\right)$ 138.0, 137.2, 136.5, 
134.0, 128.7, 128.2, 128.1, 118.8, 116.0, 107.0, 68.6, 66.6, 58.9, 55.8; IR (Neat Film $\mathrm{NaCl}): 3450,3078,2978,2929,2820,1949,1851,1688,1643,1491,1446,1362,1251$, 1081, 993, 923, 751, $696 \mathrm{~cm}^{-1}$; HRMS (MM: ESI-APCI+) m/z calc'd for $\mathrm{C}_{16} \mathrm{H}_{21} \mathrm{INO}$ $[\mathrm{M}+\mathrm{H}]^{+}:$370.0662; found: 370.0664 .<smiles>C=CC(O)CN(C/C(I)=C/c1ccccc1)CC(OCC)OCC</smiles>

1 p (283 mg, $0.635 \mathrm{mmol}$ ) was synthesized from 3,4-epoxy-1-butene (409 mg, 5.84 mmol); 11\% yield (2 steps); $\mathrm{R}_{f}=0.38$ (1:4 EtOAc:hexanes); ${ }^{1} \mathrm{H}$ NMR (500 MHz, $\left.\mathrm{CDCl}_{3}\right) \delta 7.54-7.47(\mathrm{~m}, 2 \mathrm{H}), 7.39-7.28(\mathrm{~m}, 3 \mathrm{H}), 6.99(\mathrm{~s}, 1 \mathrm{H}), 5.80(\mathrm{ddd}, J=17.2$, 10.5, 5.8 Hz, 1H), $5.34(\mathrm{dt}, J=17.2,1.6 \mathrm{~Hz}, 1 \mathrm{H}), 5.15(\mathrm{dt}, J=10.5,1.5 \mathrm{~Hz}, 1 \mathrm{H}), 4.65$ $(\mathrm{t}, J=5.4 \mathrm{~Hz}, 1 \mathrm{H}), 4.28-4.19(\mathrm{~m}, 1 \mathrm{H}), 3.95(\mathrm{~s}, 1 \mathrm{H}), 3.77-3.65(\mathrm{~m}, 3 \mathrm{H}), 3.58(\mathrm{dpd}, J$ $=9.2,7.0,2.9 \mathrm{~Hz}, 3 \mathrm{H}), 2.90-2.70(\mathrm{~m}, 3 \mathrm{H}), 2.59(\mathrm{dd}, J=13.1,10.2 \mathrm{~Hz}, 1 \mathrm{H}), 1.24(\mathrm{td}$, $J=7.0,1.0 \mathrm{~Hz}, 6 \mathrm{H}) ;{ }^{13} \mathrm{C} \mathrm{NMR}\left(126 \mathrm{MHz}, \mathrm{CDCl}_{3}\right) \delta 138.0,137.3,136.2,128.7,128.2$, 128.1, 115.9, 107.0, 101.8, 69.7, 69.3, 62.9, 62.3, 61.2, 56.5, 15.5, 15.4; IR(Neat Film $\mathrm{NaCl}) 3457,2975,2879,1686,1646,1491,1445,1374,1255,1122,1063,921,859$, 750, $696 \mathrm{~cm}^{-1}$; HRMS (MM: ESI-APCI+) m/z calc'd for $\mathrm{C}_{19} \mathrm{H}_{29} \mathrm{INO}_{3}[\mathrm{M}+\mathrm{H}]^{+}$: 446.1187; found: 446.1185 .

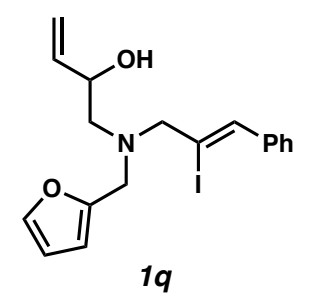


1q (306 mg, $0.748 \mathrm{mmol}$ ) was synthesized from 3,4-epoxy-1-butene (452 mg, 6.45 mmol); 12\% (2 steps); $\mathrm{R}_{f}=0.59$ (1:4 EtOAc:hexanes) $;{ }^{1} \mathrm{H} \mathrm{NMR}\left(500 \mathrm{MHz}, \mathrm{CDCl}_{3}\right) \delta$ $7.59-7.52(\mathrm{~m}, 2 \mathrm{H}), 7.44-7.29(\mathrm{~m}, 4 \mathrm{H}), 7.04(\mathrm{~s}, 1 \mathrm{H}), 6.35(\mathrm{dd}, J=3.2,1.9 \mathrm{~Hz}, 1 \mathrm{H})$, $6.23(\mathrm{dd}, J=3.2,0.8 \mathrm{~Hz}, 1 \mathrm{H}), 5.81(\mathrm{ddd}, J=17.2,10.5,5.7 \mathrm{~Hz}, 1 \mathrm{H}), 5.36(\mathrm{dt}, J=17.2$, $1.6 \mathrm{~Hz}, 1 \mathrm{H}), 5.17(\mathrm{dt}, J=10.5,1.5 \mathrm{~Hz}, 1 \mathrm{H}), 4.24(\mathrm{dddd}, J=10.3,5.7,3.1,1.5 \mathrm{~Hz}, 1 \mathrm{H})$, $3.86-3.76(\mathrm{~m}, 2 \mathrm{H}), 3.70(\mathrm{~s}, 1 \mathrm{H}), 3.60(\mathrm{dd}, J=13.9,1.4 \mathrm{~Hz}, 1 \mathrm{H}), 3.42(\mathrm{dd}, J=13.8$, $1.1 \mathrm{~Hz}, 1 \mathrm{H}), 2.72(\mathrm{dd}, J=13.0,3.2 \mathrm{~Hz}, 1 \mathrm{H}), 2.55(\mathrm{dd}, J=13.0,10.3 \mathrm{~Hz}, 1 \mathrm{H}) ;{ }^{13} \mathrm{C} \mathrm{NMR}$ $\left(126 \mathrm{MHz}, \mathrm{CDCl}_{3}\right) \delta 151.5,142.5,138.1,137.3,137.0,128.8,128.4,128.3,116.2$, 110.3, 109.4, 106.9, 68.9, 66.5, 59.3, 48.4; IR (Neat Film NaCl) 3458, 3080, 2932, 2831, 1951, 1754, 1645, 1598, 1501, 1445, 1364, 1318, 1285, 1250, 1148, 1074, 1012, 921, 750, $696 \mathrm{~cm}^{-1}$; HRMS (MM: ESI-APCI+) m/z calc'd for $\mathrm{C}_{18} \mathrm{H}_{21} \mathrm{INO}_{2}[\mathrm{M}+\mathrm{H}]^{+}$: 410.0611; found: 410.0615 .

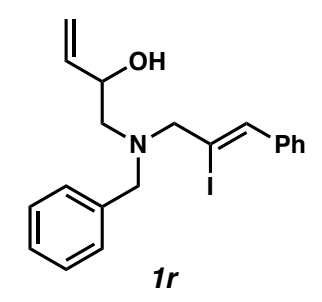

1r (334 mg, $0.797 \mathrm{mmol}$ ) was synthesized from 3,4-epoxy-1-butene (452 mg, 6.45 mmol); $12 \%$ yield (2 steps); $\mathrm{R}_{f}=0.43$ (1:4 EtOAc:hexanes); ${ }^{1} \mathrm{H}$ NMR (500 MHz, $\left.\mathrm{CDCl}_{3}\right) \delta 7.56-7.50(\mathrm{~m}, 2 \mathrm{H}), 7.41-7.27(\mathrm{~m}, 8 \mathrm{H}), 7.01(\mathrm{~s}, 1 \mathrm{H}), 5.76(\mathrm{ddd}, J=17.2$, 10.5, $5.8 \mathrm{~Hz}, 1 \mathrm{H}), 5.32(\mathrm{dt}, J=17.2,1.5 \mathrm{~Hz}, 1 \mathrm{H}), 5.14(\mathrm{dt}, J=10.5,1.4 \mathrm{~Hz}, 1 \mathrm{H}), 4.22$ $(\operatorname{tdd}, J=7.3,5.4,1.4 \mathrm{~Hz}, 1 \mathrm{H}), 3.93(\mathrm{~d}, J=13.5 \mathrm{~Hz}, 1 \mathrm{H}), 3.66(\mathrm{dd}, J=13.8,1.4 \mathrm{~Hz}$, $1 \mathrm{H}), 3.55(\mathrm{~d}, J=13.6 \mathrm{~Hz}, 2 \mathrm{H}), 3.36(\mathrm{dd}, J=13.7,0.9 \mathrm{~Hz}, 1 \mathrm{H}), 2.62-2.54(\mathrm{~m}, 2 \mathrm{H})$; ${ }^{13} \mathrm{C} \mathrm{NMR}\left(126 \mathrm{MHz}, \mathrm{CDCl}_{3}\right) \delta 138.1,137.7,137.4,137.1,129.6,128.8,128.6,128.34$, 128.25, 127.6, 116.2, 107.0, 68.9, 67.1, 59.3, 57.9; IR (Neat Film NaCl) 3460, 3082, $3060,3025,2935,2813,1950,1880,1808,1710,1645,1600,1493,1446,1364,1245$, 
1125, 1076, 991, 921, 744, $697 \mathrm{~cm}^{-1}$; HRMS (MM: ESI-APCI+) m/z calc'd for $\mathrm{C}_{20} \mathrm{H}_{23} \mathrm{INO}[\mathrm{M}+\mathrm{H}]^{+}:$: 420.0819; found: 420.0836 .<smiles>C=CC(O)CN(C/C(I)=C/c1ccccc1)Cc1ccc(OC)cc1</smiles>

1s (158 mg, $0.351 \mathrm{mmol})$ was synthesized from 3,4-epoxy-1-butene (452 mg, 6.45 mmol); 5\% (2 steps); $\mathrm{R}_{f}=0.45$ (1:4 EtOAc:hexanes); ${ }^{1} \mathrm{H}$ NMR $\left(500 \mathrm{MHz}, \mathrm{CDCl}_{3}\right) \delta$ $7.56-7.47(\mathrm{~m}, 2 \mathrm{H}), 7.39-7.27(\mathrm{~m}, 5 \mathrm{H}), 7.00(\mathrm{~s}, 1 \mathrm{H}), 6.91-6.85(\mathrm{~m}, 2 \mathrm{H}), 5.75(\mathrm{ddd}$, $J=17.3,10.5,5.8 \mathrm{~Hz}, 1 \mathrm{H}), 5.31(\mathrm{dt}, J=17.2,1.5 \mathrm{~Hz}, 1 \mathrm{H}), 5.13(\mathrm{dt}, J=10.5,1.4 \mathrm{~Hz}$, $1 \mathrm{H}), 4.24-4.15(\mathrm{~m}, 1 \mathrm{H}), 3.86(\mathrm{~d}, J=13.4 \mathrm{~Hz}, 1 \mathrm{H}), 3.81(\mathrm{~s}, 3 \mathrm{H}), 3.63(\mathrm{~d}, J=13.7 \mathrm{~Hz}$, 1H), $3.55(\mathrm{~s}, 1 \mathrm{H}), 3.49(\mathrm{~d}, J=13.4 \mathrm{~Hz}, 1 \mathrm{H}), 3.32(\mathrm{~d}, J=13.8 \mathrm{~Hz}, 1 \mathrm{H}), 2.63-2.50(\mathrm{~m}$, $2 \mathrm{H}) ;{ }^{13} \mathrm{C} \mathrm{NMR}\left(126 \mathrm{MHz}, \mathrm{CDCl}_{3}\right) \delta 158.9,138.0,137.2,136.8,130.6,129.5,128.7$, 128.2, 128.1, 116.0, 113.8, 107.0, 68.7, 66.8, 59.0, 57.1, 55.2; IR (Neat Film NaCl) 3448, 2933, 2833, 1611, 1511, 1444, 1302, 1249, 1174, 1081, 1034, 922, 845, 750, 696 $\mathrm{cm}^{-1}$; HRMS (MM: ESI-APCI + ) m/z calc'd for $\mathrm{C}_{21} \mathrm{H}_{25} \mathrm{INO}_{2}[\mathrm{M}+\mathrm{H}]^{+}:$450.0924; found: 450.0914 .

\section{Representative procedure for morpholines}
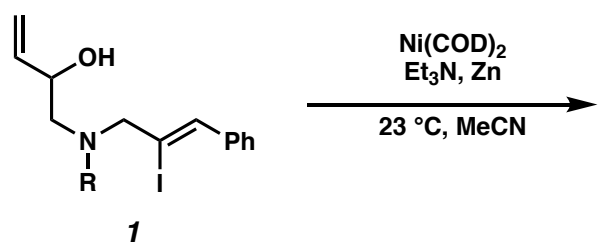<smiles>C=CC1CN(P)C/C(=C/c2ccccc2)O1</smiles>

Ni-Catalyzed $\mathrm{C}-\mathrm{O}$ bond formation experiments were performed in a nitrogen-filled glove box. To a solution of aminoalcohol 1 (0.291 mmol, 1.00 equiv) in MeCN (1.94 
$\mathrm{mL})$ in a scintillation vial were added $\mathrm{Et}_{3} \mathrm{~N}(0.320 \mathrm{mmol}, 1.10$ equiv), $\mathrm{Zn}$ powder $(0.582$ mmol, 2.00 equiv), and $\mathrm{Ni}(\mathrm{COD})_{2}(0.0146 \mathrm{mmol}, 0.05$ equiv $)$. The mixture was stirred at $23{ }^{\circ} \mathrm{C}$ for $24 \mathrm{~h}$. After the reaction was completed, the vial was removed from the glovebox and uncapped. Solids were removed via filtration through a celite plug, and the resulting solution was concentrated under reduced pressure. The residue was purified by flash column chromatography using a mixture of hexanes and ethyl acetate as eluent to furnish morpholine 2 .

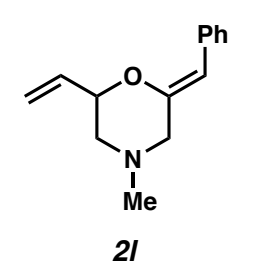

21 (149 mg, $0.692 \mathrm{mmol}$ ) was synthesized from 11 (379 mg, $1.10 \mathrm{mmol}) ; 63 \%$ yield; $\mathrm{R}_{f}$ $=0.23$ (1:2 EtOAc:hexanes); ${ }^{1} \mathrm{H}$ NMR $\left(400 \mathrm{MHz}, \mathrm{CD}_{2} \mathrm{Cl}_{2}\right) \delta 7.51(\mathrm{dd}, J=8.3,1.4 \mathrm{~Hz}$, 2H), $7.18(\mathrm{dd}, J=8.4,7.0 \mathrm{~Hz}, 2 \mathrm{H}), 7.08-7.01(\mathrm{~m}, 1 \mathrm{H}), 5.89(\mathrm{ddd}, J=17.3,10.7,5.6$ $\mathrm{Hz}, 1 \mathrm{H}), 5.40-5.32(\mathrm{~m}, 2 \mathrm{H}), 5.19-5.16(\mathrm{~m}, 1 \mathrm{H}), 4.35$ (dddt, $J=9.8,5.7,2.9,1.5 \mathrm{~Hz}$, 1H), $3.09(\mathrm{dd}, J=12.6,1.6 \mathrm{~Hz}, 1 \mathrm{H}), 2.78-2.69(\mathrm{~m}, 2 \mathrm{H}), 2.21(\mathrm{~s}, 3 \mathrm{H}), 2.08(\mathrm{dd}, J=$ 11.7, 9.5 Hz, 1H); ${ }^{13} \mathrm{C}$ NMR (101 MHz, $\left.\mathrm{CD}_{2} \mathrm{Cl}_{2}\right) \delta 150.5,136.4,136.2,128.9,128.6$, 126.4, 117.1, 107.9, 78.0, 59.5, 58.3, 46.2; IR (Neat Film NaCl) 2939, 2784, 2360, 1666, 1448, 1328, 1239, 1176, 1133, 1047, 985, 937, 755, $694 \mathrm{~cm}^{-1}$; HRMS (MM: ESIAPCI+) m/z calc'd for $\mathrm{C}_{14} \mathrm{H}_{18} \mathrm{NO}[\mathrm{M}+\mathrm{H}]^{+}: 216.1383$; found: 216.1399 .

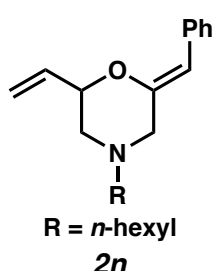

$2 n$ 
2n (262 mg, $0.918 \mathrm{mmol})$ was synthesized from 1 n (436 mg, $1.05 \mathrm{mmol}) ; 87 \%$ yield; $\mathrm{R}_{f}=0.32\left(1: 4\right.$ EtOAc:hexanes); ${ }^{1} \mathrm{H}$ NMR $\left(300 \mathrm{MHz}, \mathrm{CDCl}_{3}\right) \delta 7.63-7.57(\mathrm{~m}, 2 \mathrm{H})$, $7.27(\mathrm{dd}, J=8.4,7.1 \mathrm{~Hz}, 2 \mathrm{H}), 7.17-7.10(\mathrm{~m}, 1 \mathrm{H}), 5.95(\mathrm{ddd}, J=17.3,10.7,5.5 \mathrm{~Hz}$, $1 \mathrm{H}), 5.50(\mathrm{~d}, J=1.3 \mathrm{~Hz}, 1 \mathrm{H}), 5.46(\mathrm{dt}, J=17.3,1.5 \mathrm{~Hz}, 1 \mathrm{H}), 5.27(\mathrm{dt}, J=10.7,1.4 \mathrm{~Hz}$, 1H), 4.46 (dddt, $J=9.8,5.6,2.9,1.5 \mathrm{~Hz}, 1 \mathrm{H}), 3.30(\mathrm{dd}, J=12.6,1.6 \mathrm{~Hz}, 1 \mathrm{H}), 2.95-$ $2.83(\mathrm{~m}, 2 \mathrm{H}), 2.42-2.33(\mathrm{~m}, 2 \mathrm{H}), 2.20(\mathrm{dd}, J=11.8,9.8 \mathrm{~Hz}, 1 \mathrm{H}), 1.58-1.46(\mathrm{~m}, 2 \mathrm{H})$, $1.38-1.23(\mathrm{~m}, 6 \mathrm{H}), 0.94-0.85(\mathrm{~m}, 3 \mathrm{H}) ;{ }^{13} \mathrm{C} \mathrm{NMR}\left(101 \mathrm{MHz}, \mathrm{CDCl}_{3}\right) \delta 149.5,135.7$ $135.5,128.4,128.1,125.9,116.9,108.2,77.1,58.5,57.2,56.2,31.7,27.1,26.5,22.6$, 14.0; IR (Neat Film NaCl): 3087, 3022, 2930, 2858, 2806, 1945, 1875, 1741, 1667, 1598, 1449, 1373, 1326, 1178, 1116, 987, 939, 826, 754, $694 \mathrm{~cm}^{-1}$; HRMS (MM: ESIAPCI+) m/z calc'd for $\mathrm{C}_{19} \mathrm{H}_{28} \mathrm{NO}[\mathrm{M}+\mathrm{H}]^{+}:$286.2165; found: 286.2165 .

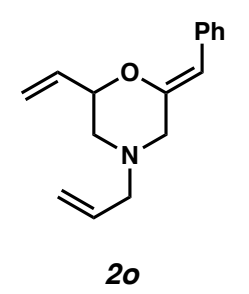

20 (161 mg, $0.667 \mathrm{mmol}$ ) was synthesized from 10 (289 mg, $0.783 \mathrm{mmol})$; 85\% yield; $\mathrm{R}_{f}=0.31$ (1:4 EtOAc:hexanes); ${ }^{1} \mathrm{H}$ NMR $\left(300 \mathrm{MHz}, \mathrm{CDCl}_{3}\right) \delta 7.65-7.57(\mathrm{~m}, 2 \mathrm{H})$, $7.33-7.24(\mathrm{~m}, 2 \mathrm{H}), 7.19-7.11(\mathrm{~m}, 1 \mathrm{H}), 6.03-5.80(\mathrm{~m}, 2 \mathrm{H}), 5.53-5.41(\mathrm{~m}, 2 \mathrm{H})$, $5.31-5.18(\mathrm{~m}, 3 \mathrm{H}), 4.51-4.41(\mathrm{~m}, 1 \mathrm{H}), 3.29(\mathrm{dd}, J=12.6,1.7 \mathrm{~Hz}, 1 \mathrm{H}), 3.05(\mathrm{dt}, J=$ 6.5, $1.3 \mathrm{~Hz}, 2 \mathrm{H}), 2.97-2.84(\mathrm{~m}, 2 \mathrm{H}), 2.21(\mathrm{dd}, J=11.8,9.7 \mathrm{~Hz}, 1 \mathrm{H}) ;{ }^{13} \mathrm{C}$ NMR $(75$ $\left.\mathrm{MHz}, \mathrm{CDCl}_{3}\right) \delta 149.3,135.6,135.4,134.1,128.4,128.1,125.9,118.8,116.9,108.3$, 77.2, 61.4, 56.9, 56.0; IR (Neat Film NaCl): 3083, 3022, 2905, 2794, 1947, 1874, 1742 , 1668, 1598, 1494, 1325, 1178, 1047, 994, 928, 827, 756, $695 \mathrm{~cm}^{-1}$; HRMS (MM: ESIAPCI+) m/z calc'd for $\mathrm{C}_{16} \mathrm{H}_{20} \mathrm{NO}[\mathrm{M}+\mathrm{H}]^{+}:$242.1539; found: 242.1543 . 
<smiles>C=CC1CN(CC(CC)OCC)CC(=Cc2ccccc2)O1</smiles>

2p (128 mg, $0.403 \mathrm{mmol}$ ) was synthesized from 1p (307 mg, $0.689 \mathrm{mmol}$ ); 58\% yield; $\mathrm{R}_{f}=0.61\left(1: 4\right.$ EtOAc:hexanes); ${ }^{1} \mathrm{H}$ NMR $\left(300 \mathrm{MHz}, \mathrm{CDCl}_{3}\right) \delta 7.65-7.55(\mathrm{~m}, 2 \mathrm{H})$, $7.34-7.21(\mathrm{~m}, 2 \mathrm{H}), 7.19-7.07(\mathrm{~m}, 1 \mathrm{H}), 5.94(\mathrm{ddd}, J=17.3,10.7,5.5 \mathrm{~Hz}, 1 \mathrm{H}), 5.52$ $-5.38(\mathrm{~m}, 2 \mathrm{H}), 5.26(\mathrm{dt}, J=10.7,1.4 \mathrm{~Hz}, 1 \mathrm{H}), 4.68(\mathrm{t}, J=5.3 \mathrm{~Hz}, 1 \mathrm{H}), 4.46(\mathrm{dddt}, J=$ 9.8, 5.7, 2.9, $1.4 \mathrm{~Hz}, 1 \mathrm{H}), 3.76-3.51(\mathrm{~m}, 4 \mathrm{H}), 3.37$ (dd, $J=12.7,1.6 \mathrm{~Hz}, 1 \mathrm{H}), 3.08-$ $2.94(\mathrm{~m}, 2 \mathrm{H}), 2.68-2.50(\mathrm{~m}, 2 \mathrm{H}), 2.38(\mathrm{dd}, J=11.9,9.7 \mathrm{~Hz}, 1 \mathrm{H}), 1.23(\mathrm{td}, J=7.1,3.6$ $\mathrm{Hz}, 6 \mathrm{H}) ;{ }^{13} \mathrm{C} \mathrm{NMR}\left(75 \mathrm{MHz}, \mathrm{CDCl}_{3}\right) \delta(\mathrm{ppm}) 149.5,135.6,135.4,128.4,128.0,125.8$, 116.8, 107.9, 101.4, 77.0, 62.0, 61.9, 60.3, 57.8, 56.6, 15.3; IR (Neat Film NaCl) 2975, 2928, 1741, 1667, 1598, 1494, 1449, 1374, 1322, 1121, 1063, 943, 847, 756, $695 \mathrm{~cm}^{-1}$; HRMS (MM: ESI-APCI+) m/z calc'd for $\mathrm{C}_{19} \mathrm{H}_{28} \mathrm{NO}_{3}[\mathrm{M}+\mathrm{H}]^{+}$: 318.2064; found: 318.2066.

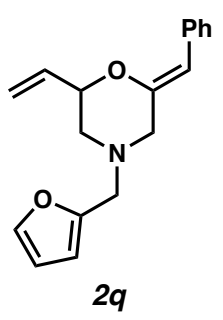

2q (187 mg, $0.665 \mathrm{mmol})$ was synthesized from 1q (298 mg, $0.728 \mathrm{mmol})$; $91 \%$ yield (2 steps); $\mathrm{R}_{f}=0.52$ (1:4 EtOAc:hexanes); ${ }^{1} \mathrm{H}$ NMR (500 MHz, $\left.\mathrm{CDCl}_{3}\right) \delta 7.61-7.57$ (m, 2H), $7.42(\mathrm{dd}, J=1.9,0.8 \mathrm{~Hz}, 1 \mathrm{H}), 7.30-7.25(\mathrm{~m}, 2 \mathrm{H}), 7.17-7.12(\mathrm{~m}, 1 \mathrm{H}), 6.36$ (dd, $J=3.2,1.8 \mathrm{~Hz}, 1 \mathrm{H}), 6.27(\mathrm{dd}, J=3.2,0.8 \mathrm{~Hz}, 1 \mathrm{H}), 5.93(\mathrm{ddd}, J=17.3,10.7,5.5$ $\mathrm{Hz}, 1 \mathrm{H}), 5.49-5.43(\mathrm{~m}, 2 \mathrm{H}), 5.27(\mathrm{dt}, J=10.7,1.4 \mathrm{~Hz}, 1 \mathrm{H}), 4.50-4.45(\mathrm{~m}, 1 \mathrm{H}), 3.62$ $(\mathrm{s}, 2 \mathrm{H}), 3.30(\mathrm{dd}, J=12.6,1.7 \mathrm{~Hz}, 1 \mathrm{H}), 3.00-2.89(\mathrm{~m}, 2 \mathrm{H}), 2.28(\mathrm{dd}, J=11.7,9.9 \mathrm{~Hz}$ 
$1 \mathrm{H}) ;{ }^{13} \mathrm{C} \mathrm{NMR}\left(126 \mathrm{MHz}, \mathrm{CDCl}_{3}\right) \delta 150.5,149.1,142.5,135.6,135.2,128.4,128.1$, 125.9, 117.0, 110.2, 109.3, 108.5, 77.0, 56.6, 55.5, 54.4; IR (Neat Film NaCl) 3086, $2935,2809,1950,1880,1737,1666,1598,1494,1450,1326,1178,1148,1040,1013$, 991, 934, 820, 756, 738, $695 \mathrm{~cm}^{-1}$; HRMS (MM: ESI-APCI+) m/z calc'd for $\mathrm{C}_{18} \mathrm{H}_{20} \mathrm{NO}_{2}$ : 282.1488; found: 282.1494 .

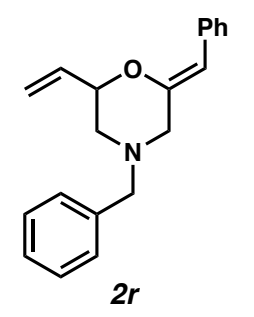

2r (136 mg, $0.467 \mathrm{mmol})$ was synthesized from 1 r (247 mg, $0.589 \mathrm{mmol}) ; 79 \%$ yield; $\mathrm{R}_{f}=0.50$ (1:4 EtOAc:hexanes); ${ }^{1} \mathrm{H}$ NMR $\left(300 \mathrm{MHz}, \mathrm{CDCl}_{3}\right) \delta 7.64-7.54(\mathrm{~m}, 2 \mathrm{H})$, $7.40-7.20(\mathrm{~m}, 7 \mathrm{H}), 7.18-7.07(\mathrm{~m}, 1 \mathrm{H}), 5.93(\mathrm{ddd}, J=17.1,10.6,5.5 \mathrm{~Hz}, 1 \mathrm{H}), 5.49$ $-5.37(\mathrm{~m}, 2 \mathrm{H}), 5.24(\mathrm{dt}, J=10.7,1.4 \mathrm{~Hz}, 1 \mathrm{H}), 4.46(\mathrm{dddt}, J=9.7,5.6,2.9,1.4 \mathrm{~Hz}, 1 \mathrm{H})$, $3.55(\mathrm{~s}, 2 \mathrm{H}), 3.25(\mathrm{dd}, J=12.6,1.6 \mathrm{~Hz}, 1 \mathrm{H}), 2.98-2.81(\mathrm{~m}, 2 \mathrm{H}), 2.23(\mathrm{dd}, J=11.7$, 9.6 Hz, $1 \mathrm{H}) ;{ }^{13} \mathrm{C} \mathrm{NMR}\left(126 \mathrm{MHz}, \mathrm{CDCl}_{3}\right) \delta(\mathrm{ppm}) 149.5,137.2,135.7,135.4,129.1$, 128.4, 128.4, 128.1, 127.4, 125.9, 116.9, 108.0, 77.1, 62.7, 56.9, 56.1; IR (Neat Film $\mathrm{NaCl}) 3085,3024,2943,2875,2805,2757,1954,1881,1812,1744,1666,1598,1493$, 1451, 1322, 1239, 1177, 1111, 1043, 826, 991, 933, 755, $696 \mathrm{~cm}^{-1}$; HRMS (MM: ESIAPCI+) m/z calc'd for $\mathrm{C}_{20} \mathrm{H}_{22} \mathrm{NO}[\mathrm{M}+\mathrm{H}]^{+}:$292.1696; found: 292.1703 .

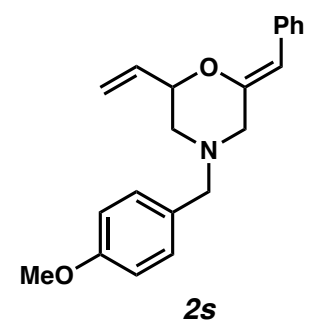


2s (128 mg, $0.398 \mathrm{mmol})$ was synthesized from $1 \mathrm{~s}$ (236 mg, $0.525 \mathrm{mmol}) ; 76 \%$ yield; $\mathrm{R}_{f}=0.52(1: 4$ EtOAc:hexanes $)=0.52 ;{ }^{1} \mathrm{H} \mathrm{NMR}\left(500 \mathrm{MHz}, \mathrm{CDCl}_{3}\right) \delta 7.69-7.63(\mathrm{~m}$, 2H), 7.33 (ddd, $J=9.5,4.4,2.0 \mathrm{~Hz}, 4 \mathrm{H}), 7.20(\mathrm{td}, J=7.3,1.3 \mathrm{~Hz}, 1 \mathrm{H}), 6.98-6.92(\mathrm{~m}$, 2H), $6.04-5.94(\mathrm{~m}, 1 \mathrm{H}), 5.54-5.46(\mathrm{~m}, 2 \mathrm{H}), 5.31(\mathrm{dt}, J=10.7,1.4 \mathrm{~Hz}, 1 \mathrm{H}), 4.52$ (dddt, $J=9.7,5.7,2.9,1.4 \mathrm{~Hz}, 1 \mathrm{H}), 3.88(\mathrm{~d}, J=1.2 \mathrm{~Hz}, 3 \mathrm{H}), 3.56(\mathrm{~d}, J=1.6 \mathrm{~Hz}, 2 \mathrm{H})$, $3.32(\mathrm{dd}, J=12.7,1.6 \mathrm{~Hz}, 1 \mathrm{H}), 2.98(\mathrm{dd}, J=12.6,1.4 \mathrm{~Hz}, 1 \mathrm{H}), 2.93(\mathrm{ddd}, J=11.7,2.9$, $1.6 \mathrm{~Hz}, 1 \mathrm{H}), 2.32-2.22(\mathrm{~m}, 1 \mathrm{H}) ;{ }^{13} \mathrm{C} \mathrm{NMR}\left(126 \mathrm{MHz}, \mathrm{CDCl}_{3}\right) \delta 158.9,149.5,135.7$, $135.4,130.3,129.1,128.4,128.1,125.8,116.8,113.7,108.0,77.1,62.1,56.8,56.0$, 55.2; IR (Neat Film NaCl) 3056, 3020, 2933, 2834, 2801, 1738, 1666, 1612, 1512, 1454 , 1324, 1289, 1249, 1177, 1106, 1036, 992, 938, 830, 756, $696 \mathrm{~cm}^{-1}$; HRMS (MM: ESIAPCI + ) m/z calc'd for $\mathrm{C}_{21} \mathrm{H}_{24} \mathrm{NO}_{2}[\mathrm{M}+\mathrm{H}]^{+}:$322.1802, Found: 322.1797.

\section{Representative procedure for Claisen rearrangement}

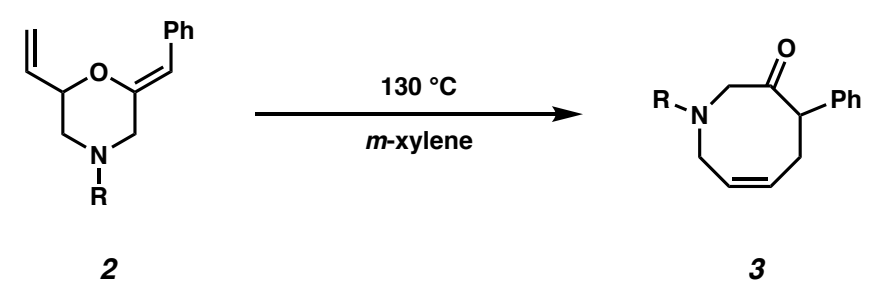

A half dram vial, equipped with a stir bar, was charged with morpholine $2(0.0929$ mmol) and $m$-xylene $(860 \mu \mathrm{L})$ in a nitrogen-filled glove box. The vial was heated at $130{ }^{\circ} \mathrm{C}$ using a heating block for $12 \mathrm{~h}$. After cooling to $23^{\circ} \mathrm{C}$, the mixture was purified using silica gel chromatography with a mixture of hexanes and ethyl acetate as eluent to afford Claisen rearrangement product 3 .

Note: Due to the COVID-19 pandemic, we are unable to conduct this experiment on a $1 \mathrm{mmol}$ scale due to limitations in our experimental lab in terms of capacity and personnel. 


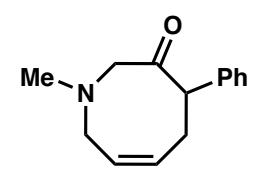

3I

31 (15.3 mg, $0.0711 \mathrm{mmol})$ was synthesized from 21 (20.0 $\mathrm{mg}, 0.0929 \mathrm{mmol})$; Note: the reaction was setup at $135{ }^{\circ} \mathrm{C}$ for $12 \mathrm{~h} ; 77 \%$ yield; $\mathrm{R}_{f}=0.39$ (1:2 EtOAc:hexanes); ${ }^{1} \mathrm{H}$ $\operatorname{NMR}\left(500 \mathrm{MHz}, \mathrm{CDCl}_{3}\right) \delta 7.46-7.41(\mathrm{~m}, 2 \mathrm{H}), 7.35(\mathrm{ddd}, J=7.7,6.8,1.2 \mathrm{~Hz}, 2 \mathrm{H})$, $7.31-7.26(\mathrm{~m}, 1 \mathrm{H}), 5.94(\mathrm{~m}, 1 \mathrm{H}), 5.61(\mathrm{dt}, J=10.8,5.1 \mathrm{~Hz}, 1 \mathrm{H}), 3.87(\mathrm{~d}, J=13.8 \mathrm{~Hz}$ 2H), $3.55(\mathrm{~d}, J=15.8 \mathrm{~Hz}, 1 \mathrm{H}), 3.42(\mathrm{~d}, J=15.3 \mathrm{~Hz}, 1 \mathrm{H}), 3.02(\mathrm{t}, J=15.3 \mathrm{~Hz}, 1 \mathrm{H}), 2.84$ $(\mathrm{d}, J=15.4 \mathrm{~Hz}, 1 \mathrm{H}), 2.48-2.43(\mathrm{~m}, 1 \mathrm{H}), 2.40(\mathrm{~s}, 3 \mathrm{H}) ;{ }^{13} \mathrm{C} \mathrm{NMR}\left(101 \mathrm{MHz}, \mathrm{CDCl}_{3}\right) \delta$ $210.7,138.3,130.9,129.0,128.7,128.1,127.6,66.1,61.3,58.1,45.9,29.1$; IR (Neat Film NaCl) 2928, 2791, 1696, 1493, 1451, 1268, 1126, $699 \mathrm{~cm}^{-1}$; HRMS (MM: ESIAPCI+) m/z calc'd for $\mathrm{C}_{14} \mathrm{H}_{18} \mathrm{NO}[\mathrm{M}+\mathrm{H}]^{+}:$216.1383; found: 216.1398 .

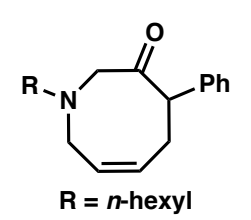

$3 n$

3n (18.2 mg, $0.0638 \mathrm{mmol})$ was synthesized from 2 n $(23.8 \mathrm{mg}, 0.0834 \mathrm{mmol}) ; 76 \%$ yield; $\mathrm{R}_{f}=0.43\left(1: 4\right.$ EtOAc:hexanes); ${ }^{1} \mathrm{H}$ NMR $\left(400 \mathrm{MHz}, \mathrm{CDCl}_{3}\right) \delta 7.36-7.14(\mathrm{~m}$, $5 \mathrm{H}), 5.83(\mathrm{dtd}, J=11.0,8.5,8.0,2.0 \mathrm{~Hz}, 1 \mathrm{H}), 5.56-5.46(\mathrm{~m}, 1 \mathrm{H}), 3.93-3.76(\mathrm{~m}, 2 \mathrm{H})$, $3.49(\mathrm{dt}, J=16.2,3.0 \mathrm{~Hz}, 1 \mathrm{H}), 3.37$ (d, $J=15.3 \mathrm{~Hz}, 1 \mathrm{H}), 2.95(\mathrm{dd}, J=16.0,5.4 \mathrm{~Hz}$, $1 \mathrm{H}), 2.80(\mathrm{~d}, J=15.3 \mathrm{~Hz}, 1 \mathrm{H}), 2.38(\mathrm{td}, J=7.7,6.9,5.3 \mathrm{~Hz}, 3 \mathrm{H}), 1.48-1.32(\mathrm{~m}, 2 \mathrm{H})$, $1.32-1.15(\mathrm{~m}, 6 \mathrm{H}), 0.82(\mathrm{t}, J=6.8 \mathrm{~Hz}, 3 \mathrm{H}) ;{ }^{13} \mathrm{C} \mathrm{NMR}\left(101 \mathrm{MHz}, \mathrm{CDCl}_{3}\right) \delta 211.1$, $138.1,130.2,128.6,128.1,127.8,127.3,64.4,60.5,57.4,55.6,31.7,28.6,27.1,27.0$ 
22.6, 14.0; IR (Neat Film NaCl): 3023, 1703, 1494, 1454, 1376, 1262, 1163, 1031, 768, 723, $700 \mathrm{~cm}^{-1}$; HRMS (MM: ESI-APCI+) m/z calc'd for $\mathrm{C}_{19} \mathrm{H}_{28} \mathrm{NO}[\mathrm{M}+\mathrm{H}]^{+}: 286.2165$; found: 286.2191 .

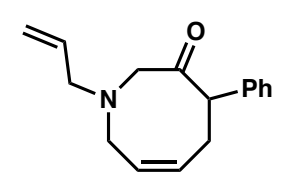

30

3o (15.2 mg, $0.0630 \mathrm{mmol})$ was synthesized from 20 (26.7 mg, $0.111 \mathrm{mmol}) ; 57 \%$ yield; $\mathrm{R}_{f}=0.38$ (1:4 EtOAc:hexanes); ${ }^{1} \mathrm{H}$ NMR $\left(500 \mathrm{MHz}, \mathrm{CDCl}_{3}\right) \delta 7.43-7.38(\mathrm{~m}, 2 \mathrm{H})$, $7.34(\mathrm{dd}, J=8.4,6.8 \mathrm{~Hz}, 2 \mathrm{H}), 7.31-7.25(\mathrm{~m}, 1 \mathrm{H}), 5.96-5.80(\mathrm{~m}, 2 \mathrm{H}), 5.58(\mathrm{dt}, J=$ 10.4, 4.9 Hz, 1H), 5.23 - 5.12 (m, 2H), $4.00-3.91(\mathrm{~m}, 1 \mathrm{H}), 3.91-3.85(\mathrm{~m}, 1 \mathrm{H}), 3.57$ (dt, $J=16.0,3.3 \mathrm{~Hz}, 1 \mathrm{H}), 3.46(\mathrm{~d}, J=15.5 \mathrm{~Hz}, 1 \mathrm{H}), 3.10(\mathrm{t}, J=5.0 \mathrm{~Hz}, 2 \mathrm{H}), 3.02$ (dd, $J=16.0,5.4 \mathrm{~Hz}, 1 \mathrm{H}), 2.86(\mathrm{~d}, J=15.4 \mathrm{~Hz}, 1 \mathrm{H}), 2.47(\mathrm{ddd}, J=11.4,7.8,5.4 \mathrm{~Hz}, 1 \mathrm{H})$; ${ }^{13} \mathrm{C}$ NMR $\left(101 \mathrm{MHz}, \mathrm{CDCl}_{3}\right) \delta 211.1,138.1,134.8,130.2,128.7,128.5,128.1,127.8$, 127.3, 118.6, 63.9, 60.5, 55.6, 28.7; IR (Neat Film NaCl) 3023, 2919, 2804, 1701, 1493 , 1450, 1327, 1265, 1164, 993, 769, $700 \mathrm{~cm}^{-1}$; HRMS (MM: ESI-APCI+) m/z calc'd for $\mathrm{C}_{16} \mathrm{H}_{20} \mathrm{NO}[\mathrm{M}+\mathrm{H}]^{+}:$242.1539; found: 242.1537 .

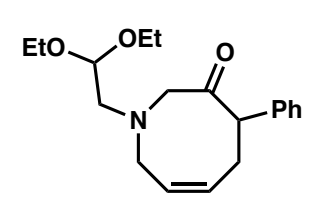

$3 p$

$3 \mathbf{p}(8.80 \mathrm{mg}, 0.0280 \mathrm{mmol})$ was synthesized from $\mathbf{2 p}(24.8 \mathrm{mg}, 0.0781 \mathrm{mmol}) ; 35 \%$ yield; $\mathrm{R}_{f}=0.50(1: 4$ EtOAc:hexanes $) ;{ }^{1} \mathrm{H} \mathrm{NMR}\left(500 \mathrm{MHz}, \mathrm{CDCl}_{3}\right) \delta 7.44(\mathrm{dd}, J=7.5$, $1.6 \mathrm{~Hz}, 2 \mathrm{H}), 7.34(\mathrm{dd}, J=8.4,6.8 \mathrm{~Hz}, 2 \mathrm{H}), 7.30-7.23(\mathrm{~m}, 1 \mathrm{H}), 5.94-5.85(\mathrm{~m}, 1 \mathrm{H})$, $5.56(\mathrm{~m}, 1 \mathrm{H}), 4.62(\mathrm{t}, J=5.2 \mathrm{~Hz}, 1 \mathrm{H}), 3.98(\mathrm{td}, J=11.7,9.0 \mathrm{~Hz}, 1 \mathrm{H}), 3.90(\mathrm{dd}, J=11.6$, $5.8 \mathrm{~Hz}, 1 \mathrm{H}), 3.73-3.42(\mathrm{~m}, 6 \mathrm{H}), 3.20(\mathrm{dd}, J=16.2,5.2 \mathrm{~Hz}, 1 \mathrm{H}), 3.03(\mathrm{~d}, J=15.6 \mathrm{~Hz}$, 1H), $2.75-2.63(\mathrm{~m}, 2 \mathrm{H}), 2.49(\mathrm{ddd}, J=11.8,7.7,5.9 \mathrm{~Hz}, 1 \mathrm{H}), 1.22(\mathrm{dt}, J=15.3,7.1$ 
$\mathrm{Hz}, 6 \mathrm{H}) ;{ }^{13} \mathrm{C} \mathrm{NMR}\left(126 \mathrm{MHz}, \mathrm{CDCl}_{3}\right) \delta 211.5,138.3,130.1,128.7,128.2,127.9,127.3$, 101.2, 65.2, 62.2, 61.8, 60.4, 59.7, 56.5, 28.7, 15.4, 15.4; IR (Neat Film NaCl) 3023, 2974, 2927, 1702, 1599, 1452, 1374, 1269, 1124, 1064, 769, $700 \mathrm{~cm}^{-1}$; HRMS (MM: ESI-APCI+) m/z calc'd for $\mathrm{C}_{19} \mathrm{H}_{28} \mathrm{NO}_{3}[\mathrm{M}+\mathrm{H}]^{+}:$318.2064; found: 318.2093.

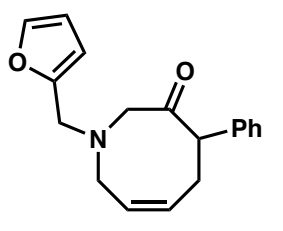

$3 q$

3q (12.2 mg, $0.0434 \mathrm{mmol})$ was synthesized from 2 q (23.5 mg, $0.0835 \mathrm{mmol}) ; 52 \%$ yield; $\mathrm{R}_{f}=0.45$ (1:4 EtOAc:hexanes); ${ }^{1} \mathrm{H}$ NMR (400 MHz, $\left.\mathrm{CDCl}_{3}\right) \delta 7.37-7.28(\mathrm{~m}$, $3 \mathrm{H}), 7.26-7.21(\mathrm{~m}, 2 \mathrm{H}), 7.20-7.14(\mathrm{~m}, 1 \mathrm{H}), 6.25(\mathrm{dd}, J=3.2,1.9 \mathrm{~Hz}, 1 \mathrm{H}), 6.18-$ $6.12(\mathrm{~m}, 1 \mathrm{H}), 5.89-5.77(\mathrm{~m}, 1 \mathrm{H}), 5.50(\mathrm{~m}, 1 \mathrm{H}), 4.00-3.87(\mathrm{~m}, 1 \mathrm{H}), 3.79(\mathrm{dd}, J=$ 12.0, $5.9 \mathrm{~Hz}, 1 \mathrm{H}), 3.62-3.44(\mathrm{~m}, 4 \mathrm{H}), 3.00(\mathrm{dd}, J=16.0,5.4 \mathrm{~Hz}, 1 \mathrm{H}), 2.82(\mathrm{~d}, J=$ 15.6 Hz, 1H), $2.35(\mathrm{ddd}, J=12.0,7.7,5.9 \mathrm{~Hz}, 1 \mathrm{H}) ;{ }^{13} \mathrm{C} \mathrm{NMR}\left(101 \mathrm{MHz}, \mathrm{CDCl}_{3}\right) \delta$ $210.9,151.5,142.4,138.1,130.4,128.7,127.9,127.8,127.3,110.1,109.3,63.6,60.9$, 55.6, 53.7, 28.9; IR (Neat Film NaCl) 3024, 2924, 2809, 1698, 1494, 1450, 1336, 1241 , 1147, 1012, 947, 916, 737, $700 \mathrm{~cm}^{-1}$; HRMS (MM: ESI-APCI+) m/z calc'd for $\mathrm{C}_{18} \mathrm{H}_{20} \mathrm{NO}_{2}[\mathrm{M}+\mathrm{H}]^{+}:$282.1489; found: 282.1496 .

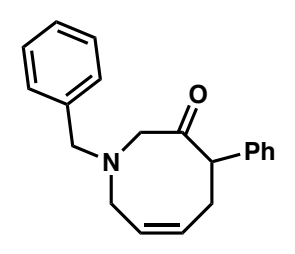

$3 r$

3r (15.2 mg, $0.0522 \mathrm{mmol})$ was synthesized from 2 r (24.9 mg, $0.0854 \mathrm{mmol}) ; 61 \%$ yield; $\mathrm{R}_{f}=0.34$ (1:4 EtOAc:hexanes); ${ }^{1} \mathrm{H}$ NMR (500 MHz, $\left.\mathrm{CDCl}_{3}\right) \delta 7.40-7.20(\mathrm{~m}$, $10 \mathrm{H}), 5.90(\mathrm{~m}, 1 \mathrm{H}), 5.56(\mathrm{~m}, 1 \mathrm{H}), 4.08-3.97(\mathrm{~m}, 1 \mathrm{H}), 3.89(\mathrm{dd}, J=11.7,6.0 \mathrm{~Hz}, 1 \mathrm{H})$, $3.66-3.50(\mathrm{~m}, 4 \mathrm{H}), 3.04(\mathrm{dd}, J=16.0,5.4 \mathrm{~Hz}, 1 \mathrm{H}), 2.89(\mathrm{~d}, J=14.9 \mathrm{~Hz}, 1 \mathrm{H}), 2.53$ 
(ddd, $J=12.2,7.7,6.0 \mathrm{~Hz}, 1 \mathrm{H}) ;{ }^{13} \mathrm{C} \mathrm{NMR}\left(101 \mathrm{MHz}, \mathrm{CDCl}_{3}\right) \delta 210.9,138.3,137.9$, $129.8,129.3,128.7,128.4,128.2,127.7,127.4,127.2,64.5,62.1,60.5,56.2,28.9$; IR (Neat Film NaCl) 3060, 3026, 2922, 2806, 1699, 1953, 1880, 1494, 1453, 1310, 1161 , 1109, 949, 733, $699 \mathrm{~cm}^{-1}$; HRMS (MM: ESI-APCI+) m/z calc'd for $\mathrm{C}_{20} \mathrm{H}_{22} \mathrm{NO}[\mathrm{M}+\mathrm{H}]^{+}$: 292.1696; found: 292.1721.

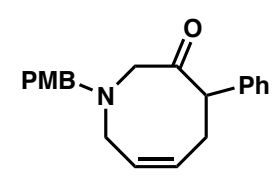

$3 s$

3s (20.0 mg, $0.0622 \mathrm{mmol})$ was synthesized from $2 \mathrm{~s}(25.1 \mathrm{mg}, 0.0781 \mathrm{mmol}) ; 80 \%$ yield; $\mathrm{R}_{f}=0.32\left(1: 4\right.$ EtOAc:hexanes); ${ }^{1} \mathrm{H}$ NMR $\left(500 \mathrm{MHz}, \mathrm{CDCl}_{3}\right) \delta 7.37-7.23(\mathrm{~m}$, $7 \mathrm{H}), 6.93-6.87(\mathrm{~m}, 2 \mathrm{H}), 5.92(\mathrm{~m}, 1 \mathrm{H}), 5.59(\mathrm{~m}, 1 \mathrm{H}), 4.04(\mathrm{td}, J=12.0,8.9 \mathrm{~Hz}, 1 \mathrm{H})$, $3.93-3.88(\mathrm{~m}, 1 \mathrm{H}), 3.85(\mathrm{~s}, 3 \mathrm{H}), 3.64-3.51(\mathrm{~m}, 4 \mathrm{H}), 3.05(\mathrm{dd}, J=16.3,5.3 \mathrm{~Hz}, 1 \mathrm{H})$, $2.59-2.50(\mathrm{~m}, 1 \mathrm{H}), 2.58-2.52(\mathrm{~m}, 1 \mathrm{H}) ;{ }^{13} \mathrm{C} \mathrm{NMR}\left(126 \mathrm{MHz}, \mathrm{CDCl}_{3}\right) \delta 211.1,158.9$, $138.3,130.5,130.0,129.6,128.6,128.3,127.7,127.2,113.7,64.4,61.5,60.5,56.2$, 55.3, 28.9; IR (Neat Film NaCl) 3023, 2931, 2835, 2807, 1699, 1611, 1512, 1453, 1302 , 1247, 1174, 1104, 1034, 832, 760, $700 \mathrm{~cm}^{-1}$; HRMS (MM: ESI-APCI + ) m/z calc'd for $\mathrm{C}_{21} \mathrm{H}_{24} \mathrm{NO}_{2}[\mathrm{M}+\mathrm{H}]^{+}:$: 322.1802; found: 322.1828 . 


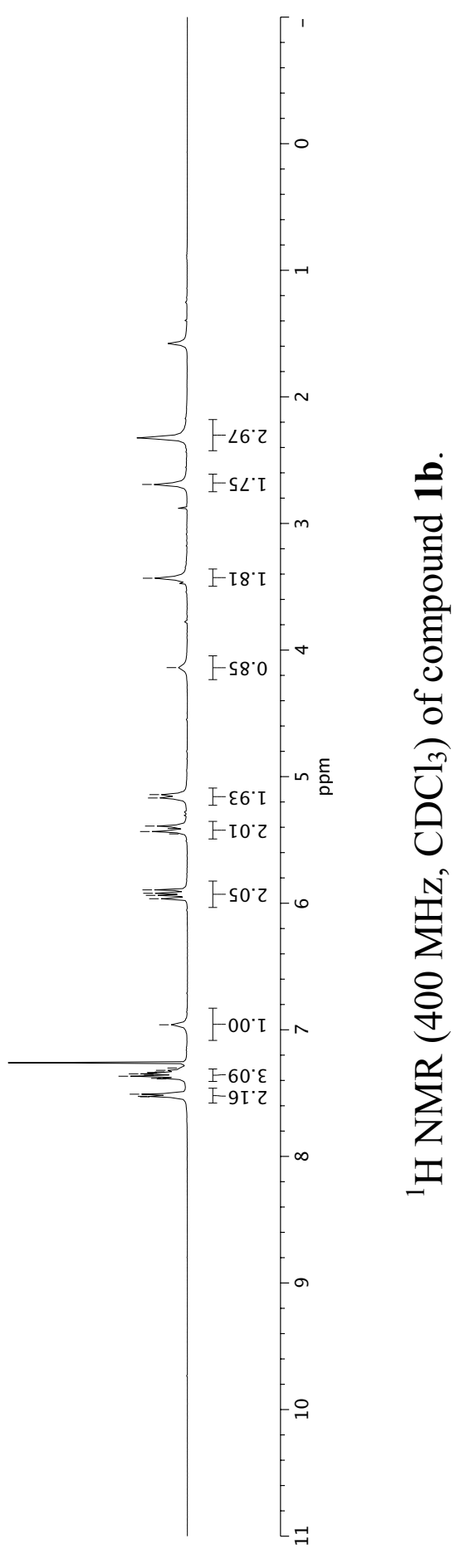




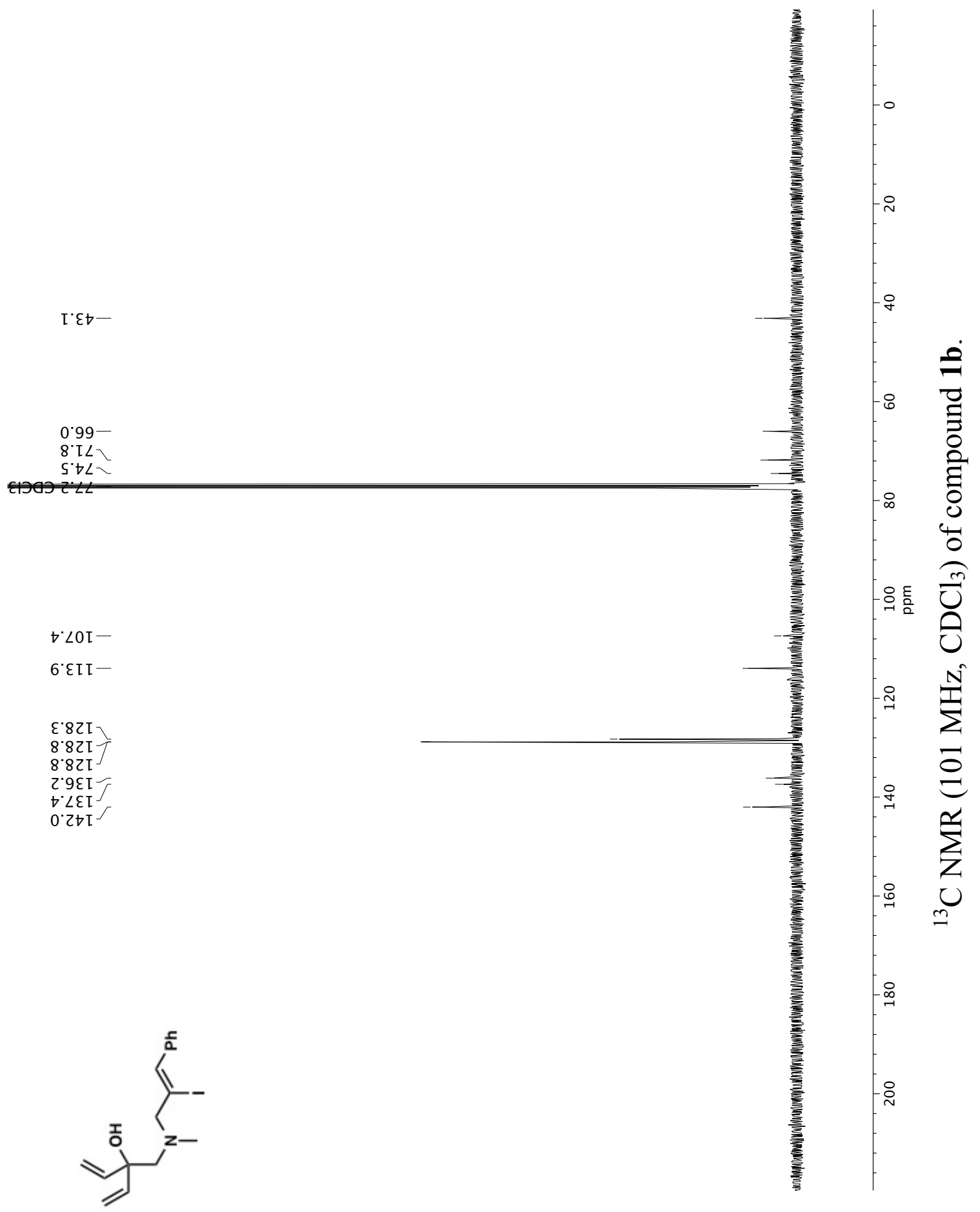



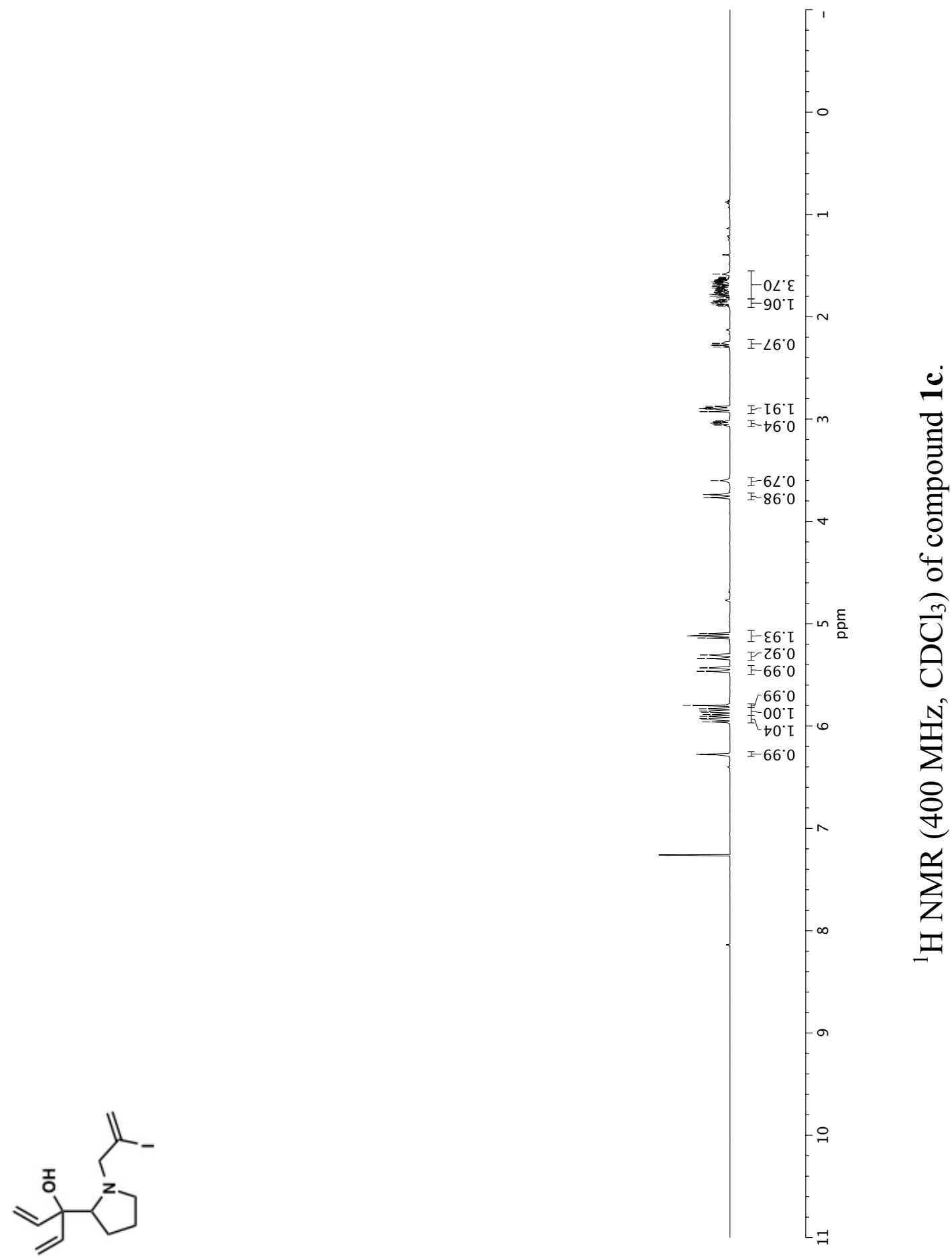
$s \cdot t z$

$6<2-$

$9 \cdot \downarrow s-$

8.29

عاده $\tau^{\prime} L L$

t'ZI I -

乙'घI I-

¿धI J

L'SZI-

[.0t I-

S.ZtI

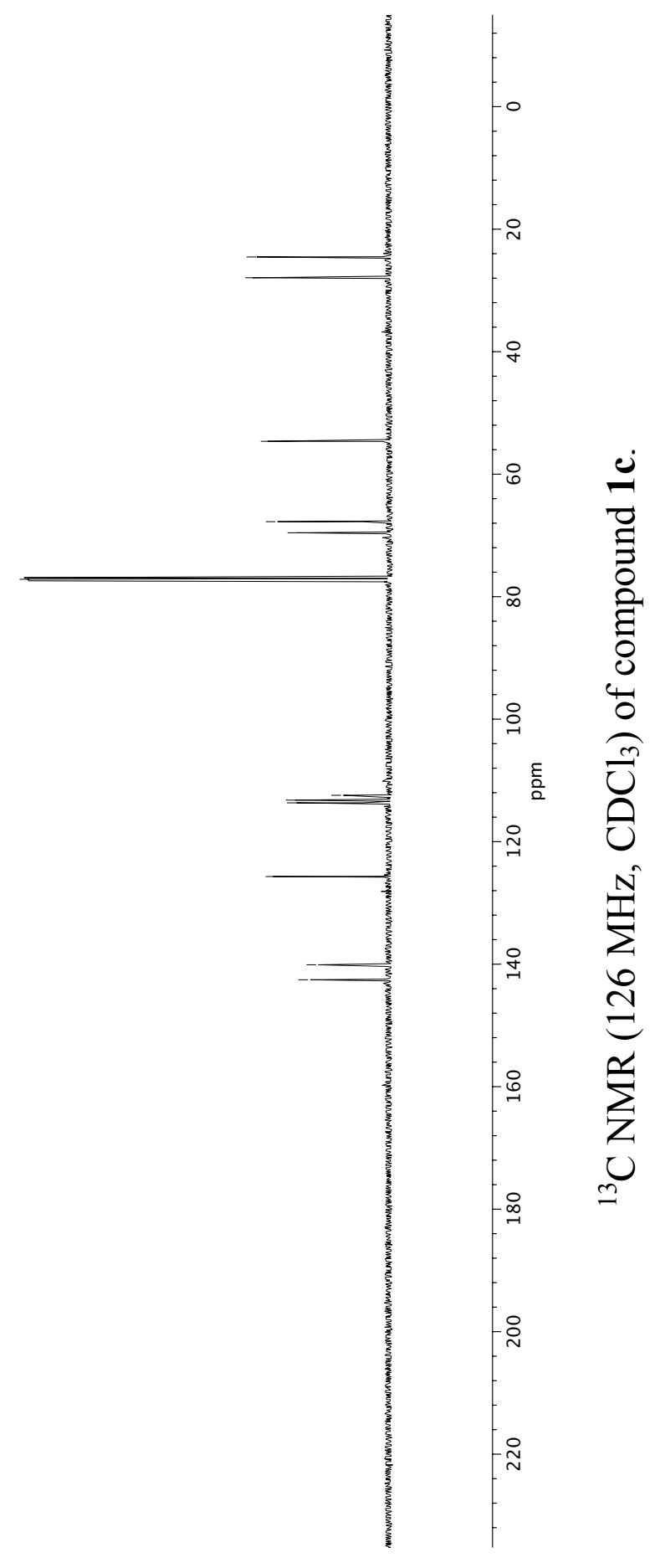


$\mathrm{L} L^{\prime} \mathrm{L}$
$\mathrm{ZL} \cdot \mathrm{L}$

$\downarrow z \cdot z-$

29'乙-

$9 \tau^{\circ} \varepsilon-$
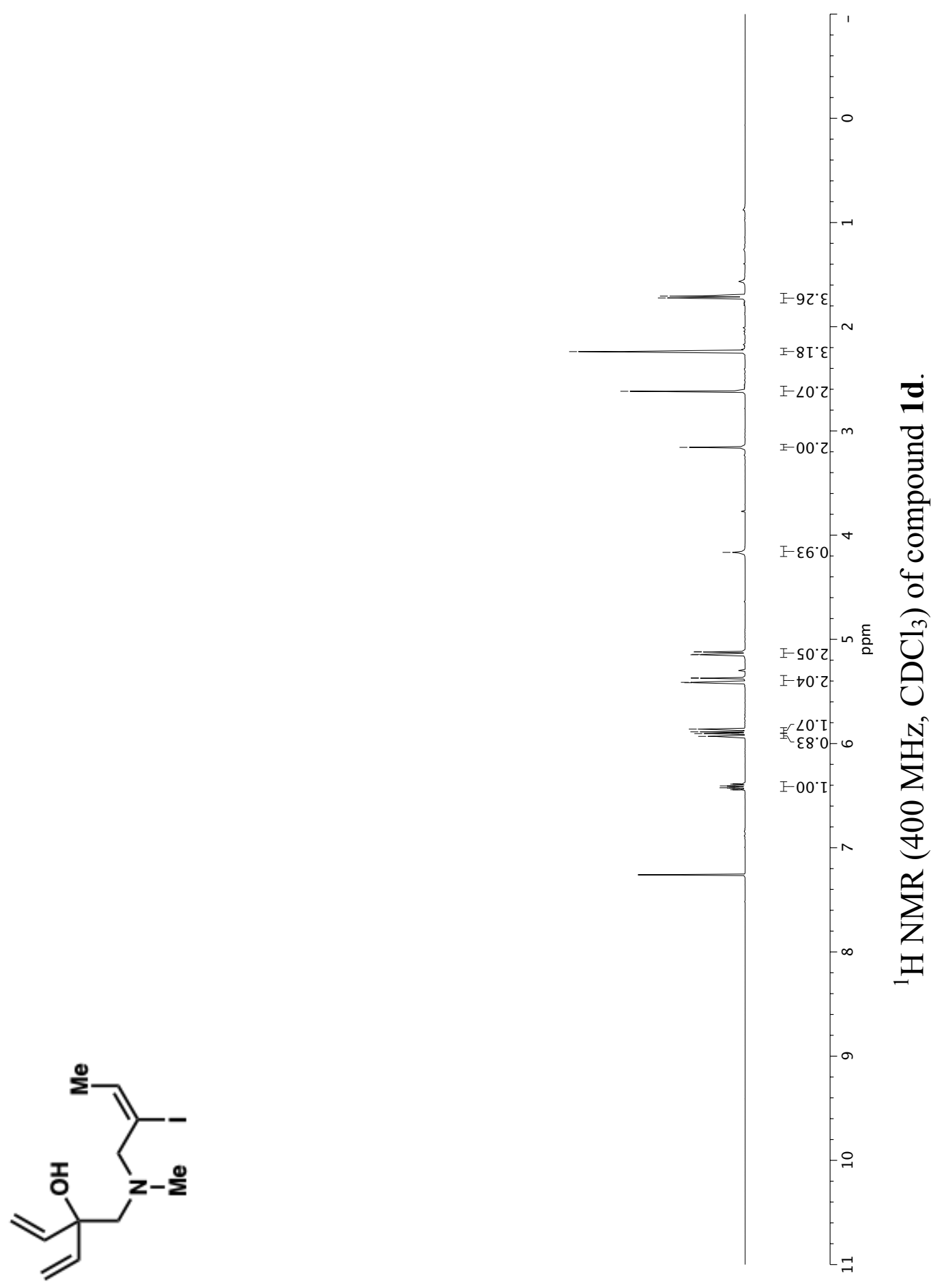


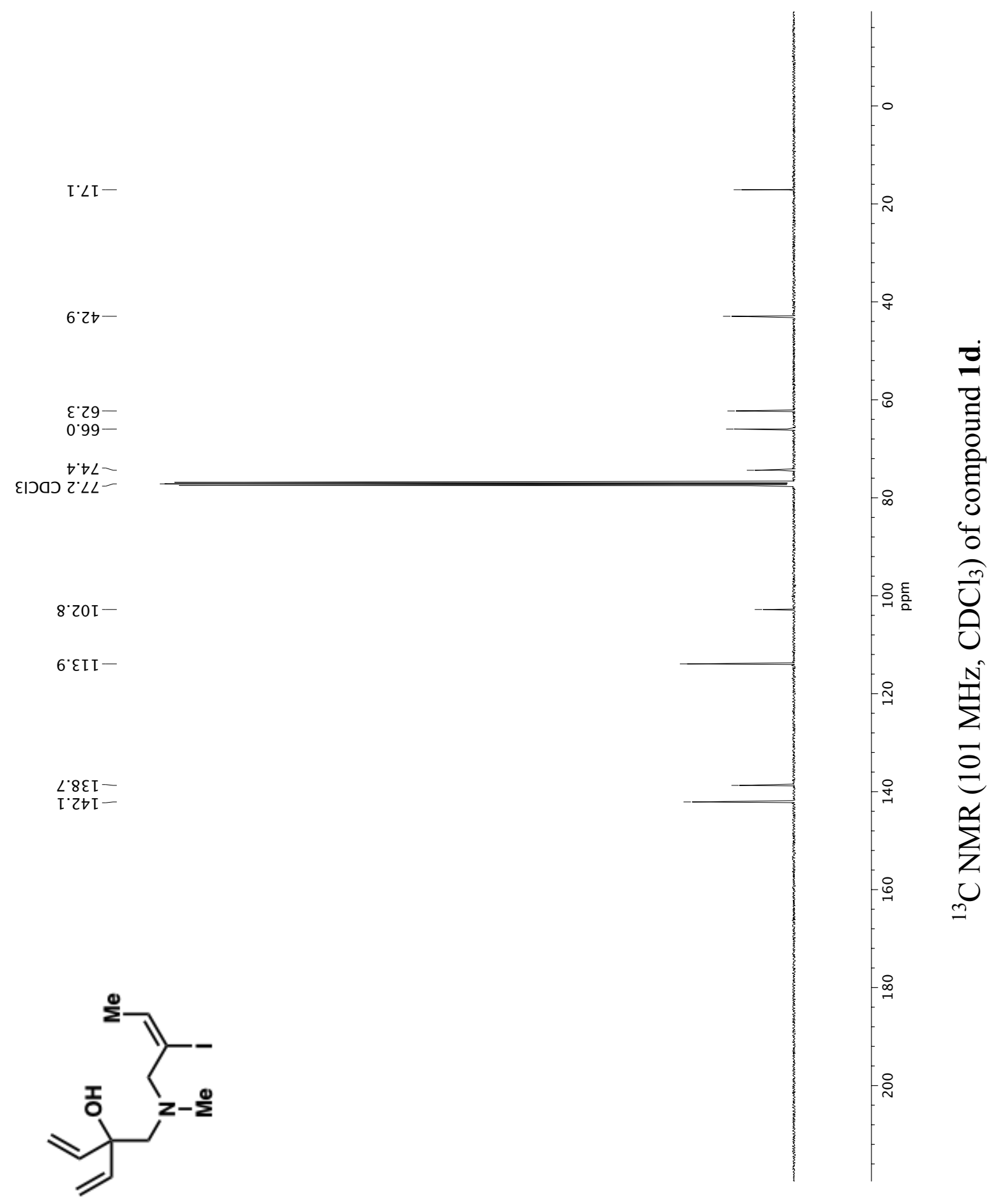



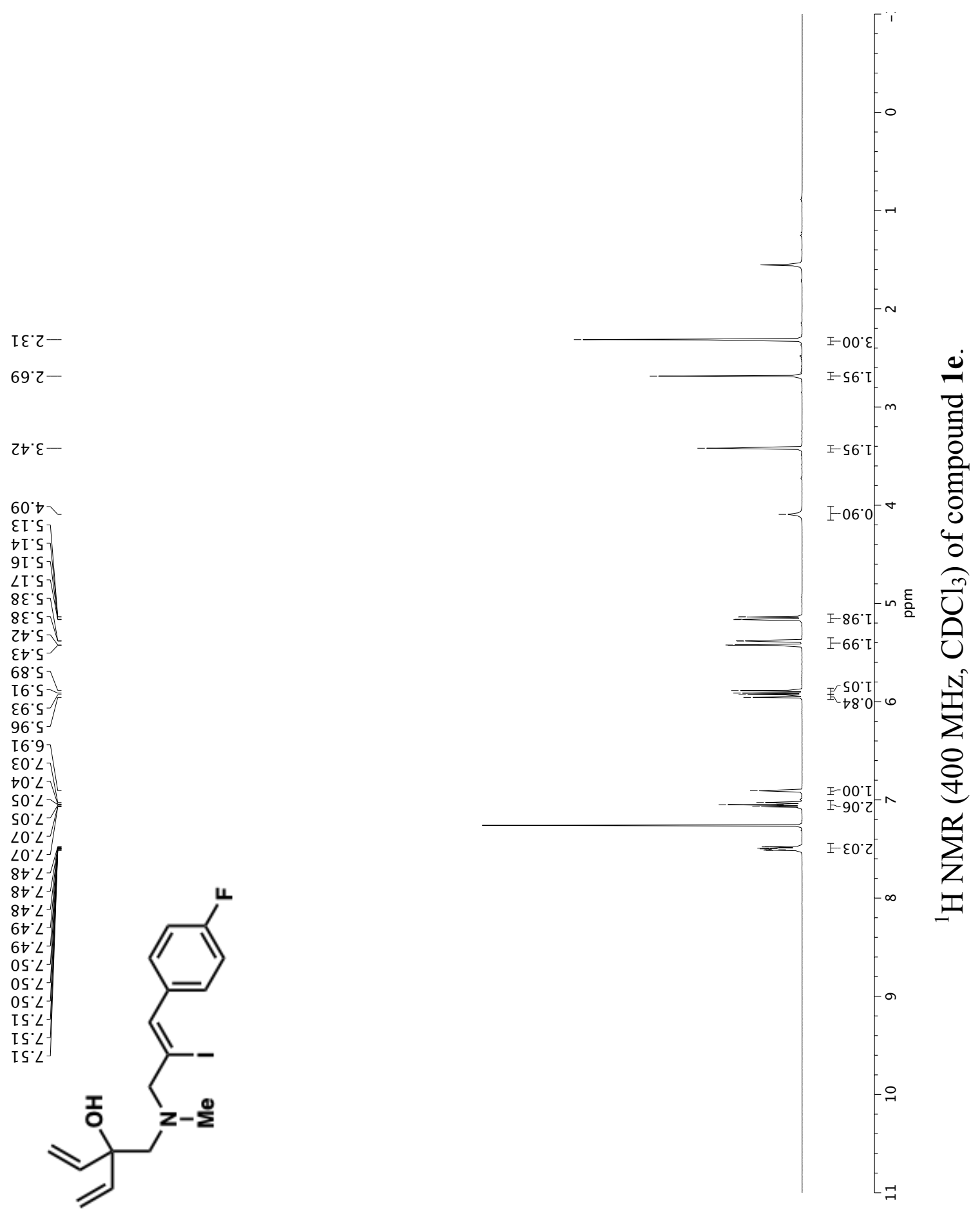
$6 I^{\circ} \varepsilon \downarrow-$

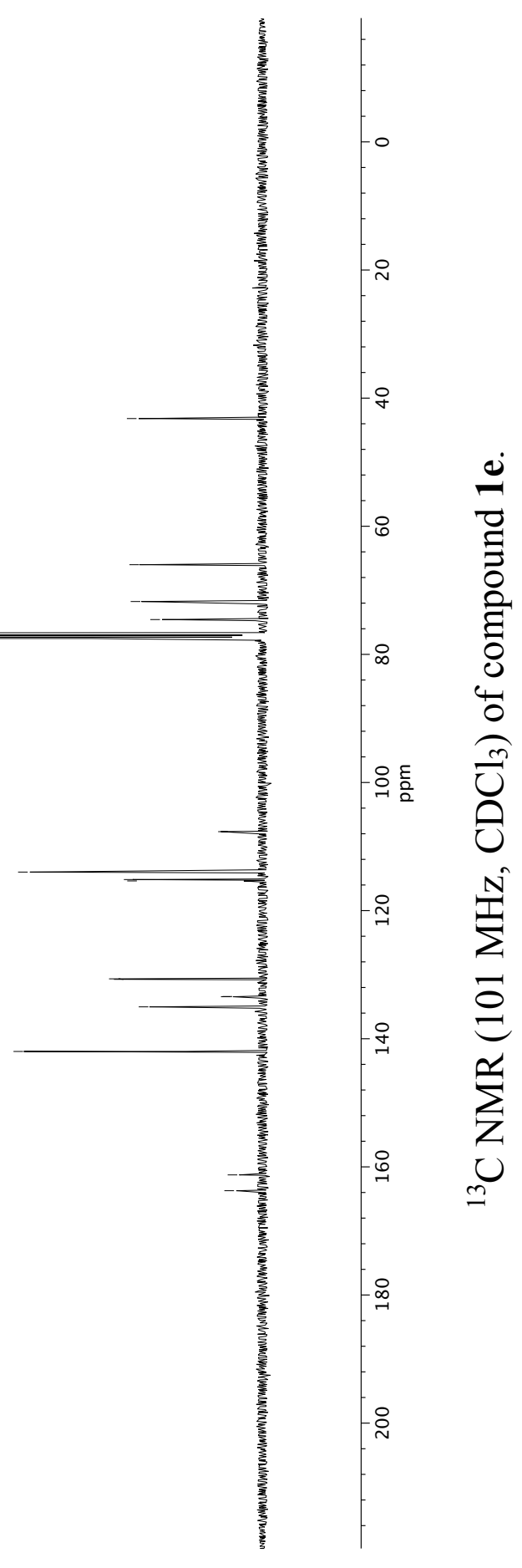

$\angle 9^{\circ} \angle O$ I-

$86^{\circ} \varepsilon \mathrm{II}$

SI'SI I -

ร9.0\& I

$\varepsilon L^{\circ} 0 \varepsilon \mathrm{I}-$

I ${ }^{\circ} \varepsilon \varepsilon \mathrm{L}-$

$\forall \overbrace{}^{\circ} \varepsilon \varepsilon \mathrm{I}$

Z0'

$86^{\circ} \mathrm{L} t \mathrm{I}$

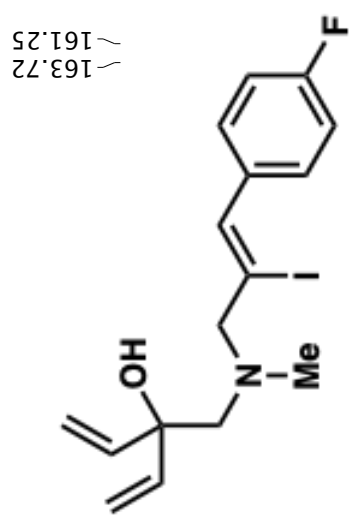

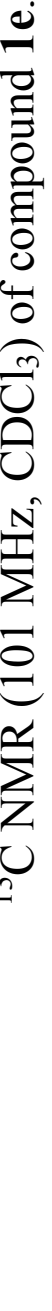


$927-$

¿9' - -

$\left.\rightarrow I^{\cdot} \varepsilon\right\rangle$

$\varepsilon 0^{\circ} \downarrow-$

ZI'S

$\varepsilon I^{\prime} S-$

SI'S

$9 \varepsilon^{*} \varsigma$

$\angle E^{\circ} \mathrm{S}$

It. $\mathrm{S}$

[ $t \cdot \mathrm{S}$

$98^{\circ} \mathrm{s}$

$98^{\circ} \mathrm{S}$

$98^{\circ} \mathrm{s}$

$68^{\circ} \mathrm{S}$

$06^{\circ} \mathrm{S}$

$\varepsilon 6^{\circ} \mathrm{s}$

$92^{\circ} 9$

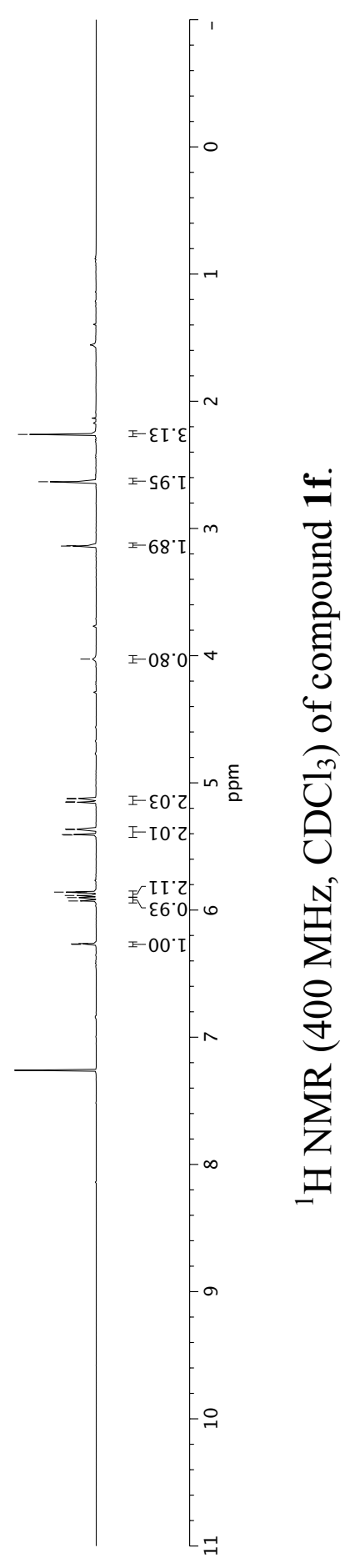




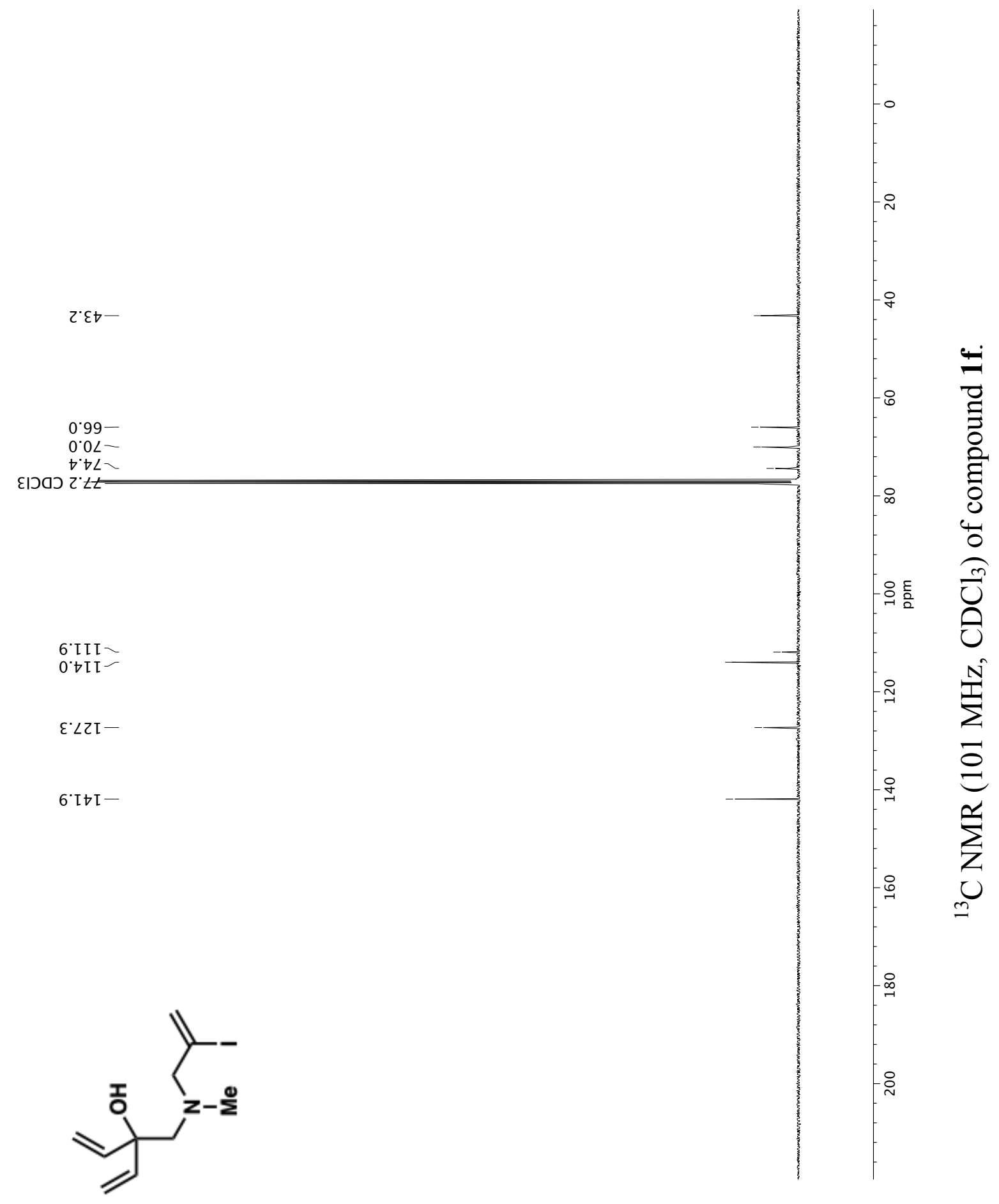


ह8. ${ }^{\circ}-5$

$\angle 2 \cdot 2$

$09^{\circ} 2$

$0 \nabla^{\cdot} \varepsilon-$

OI'

I I'S ]

$\varepsilon I^{\prime} S-$

$\varepsilon I^{\prime} S$

$\varsigma \varepsilon^{\prime} \varsigma$

ऽ. $\varepsilon^{\circ}-$

$8 \varepsilon^{\circ} \mathrm{S}$

$6 \varepsilon^{\circ} \mathrm{S}$

$18^{\circ} \mathrm{S}$

68.5

$06^{\circ} \mathrm{S}$

$26^{\circ} \mathrm{s}$
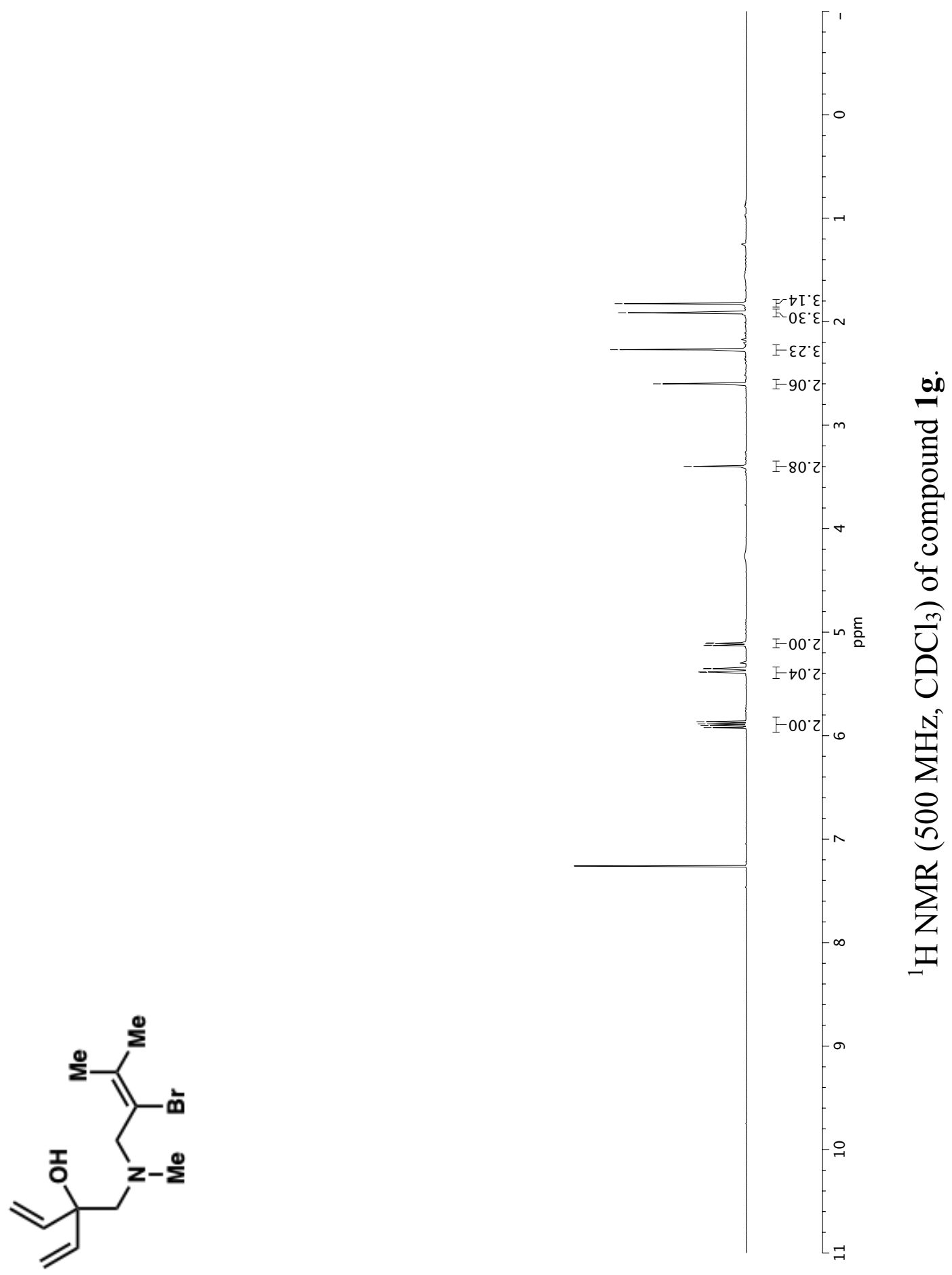


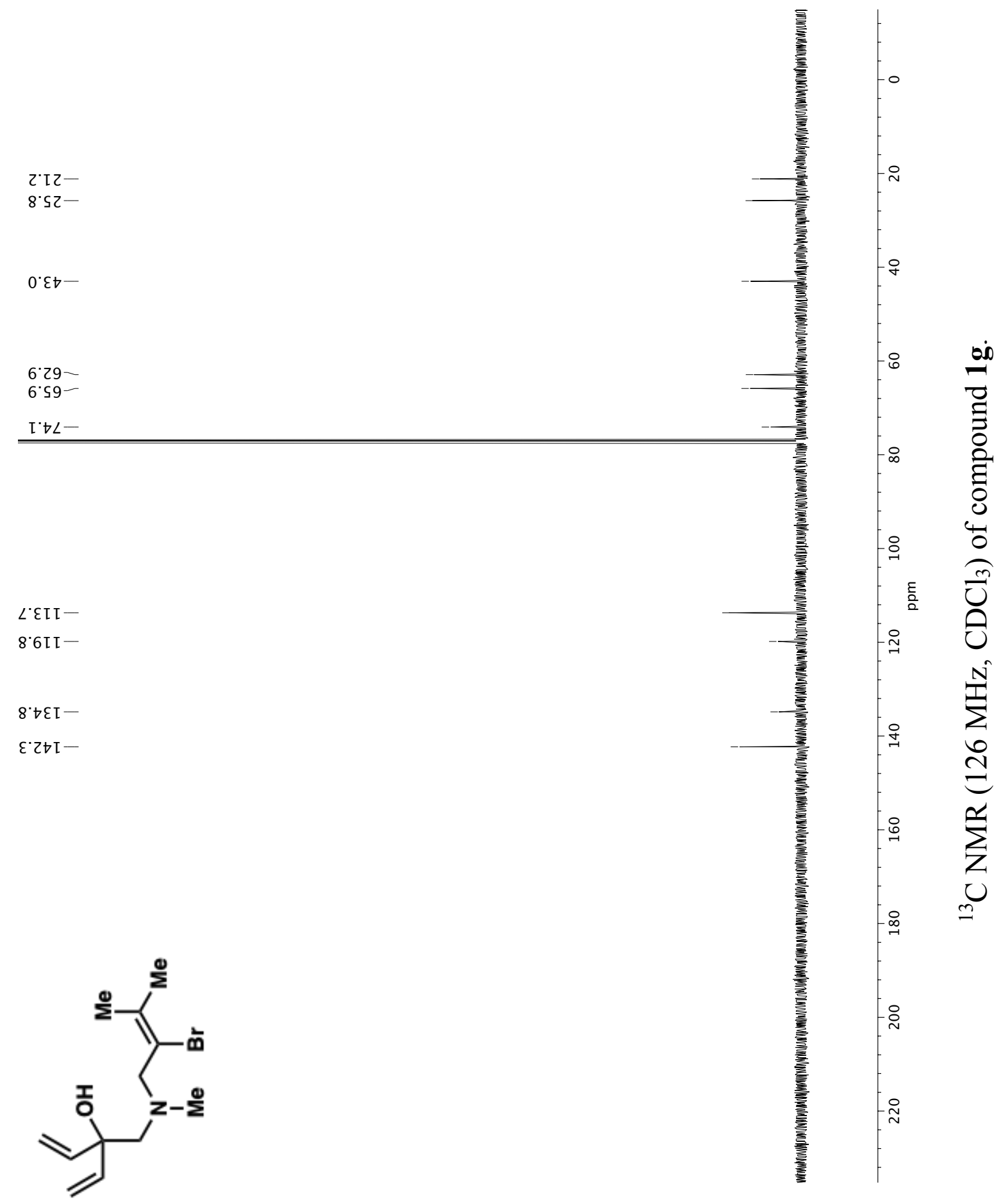


$\angle 26 I^{\circ} Z-$

$0966^{\circ} \mathrm{Z}$

[ $\angle 00^{\circ} \varepsilon$

$\varepsilon 6 \angle Z^{\circ} \varepsilon$

$\angle\left[82^{\circ} \varepsilon\right]$

乙90ध $\varepsilon-$

$980 \varepsilon^{\circ} \varepsilon-$

ธ6โt'E-

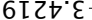

$\rightarrow 9 t t^{\circ} \varepsilon$

$88+t{ }^{\circ} \varepsilon$

$\varepsilon 6 t I^{\circ} \varsigma$

ZZSI'S

$\nabla 0 \angle I^{\circ} \mathrm{S}$

$\forall \varepsilon<T \cdot S$

$9 \forall \forall t \cdot \mathrm{S}$

$\mathrm{s} \angle t t \cdot \mathrm{s}$

$\angle 8 \angle t^{\circ} \mathrm{S}$

$\angle \mathrm{I} 8 \mathrm{t}^{\circ} \mathrm{S}$

I I $8 \mathrm{I}^{\circ} 9$

zZ0z' 9

ZSIZ' 9

Łaิรz: 9

$6 Z \triangleright Z^{\circ} L$

$S \angle S Z^{\circ} \angle$

I092 $\angle$

$60 z \varepsilon^{\circ} \angle$

$6 \sqcup \varepsilon \varepsilon \cdot<-$

$8 \angle \varepsilon \varepsilon<-$

$\angle 8 \nabla \varepsilon^{\circ} \angle$

ZOSE. $L$

$\downarrow \varepsilon \varsigma \varepsilon^{\circ} L$

SI9E. $\angle$

6 † $9 \varepsilon^{\circ} \angle$

$\angle 89 \varepsilon^{\circ} \angle$

$88 \angle \varepsilon^{\circ} \angle$

$8 S 6 t^{\circ} L$

$\angle \angle 6 t^{\circ} \angle$

$966 t^{\circ} \angle$

SIOS' $\angle$

$\rightarrow 605^{\circ} \mathrm{L}$

IEIS $L$

$\angle S I S . \angle$

$9 \angle \mathrm{IS} \angle$

S6IS.

$8625^{\circ} \mathrm{L}$

$\angle Z E S^{\circ} \angle$

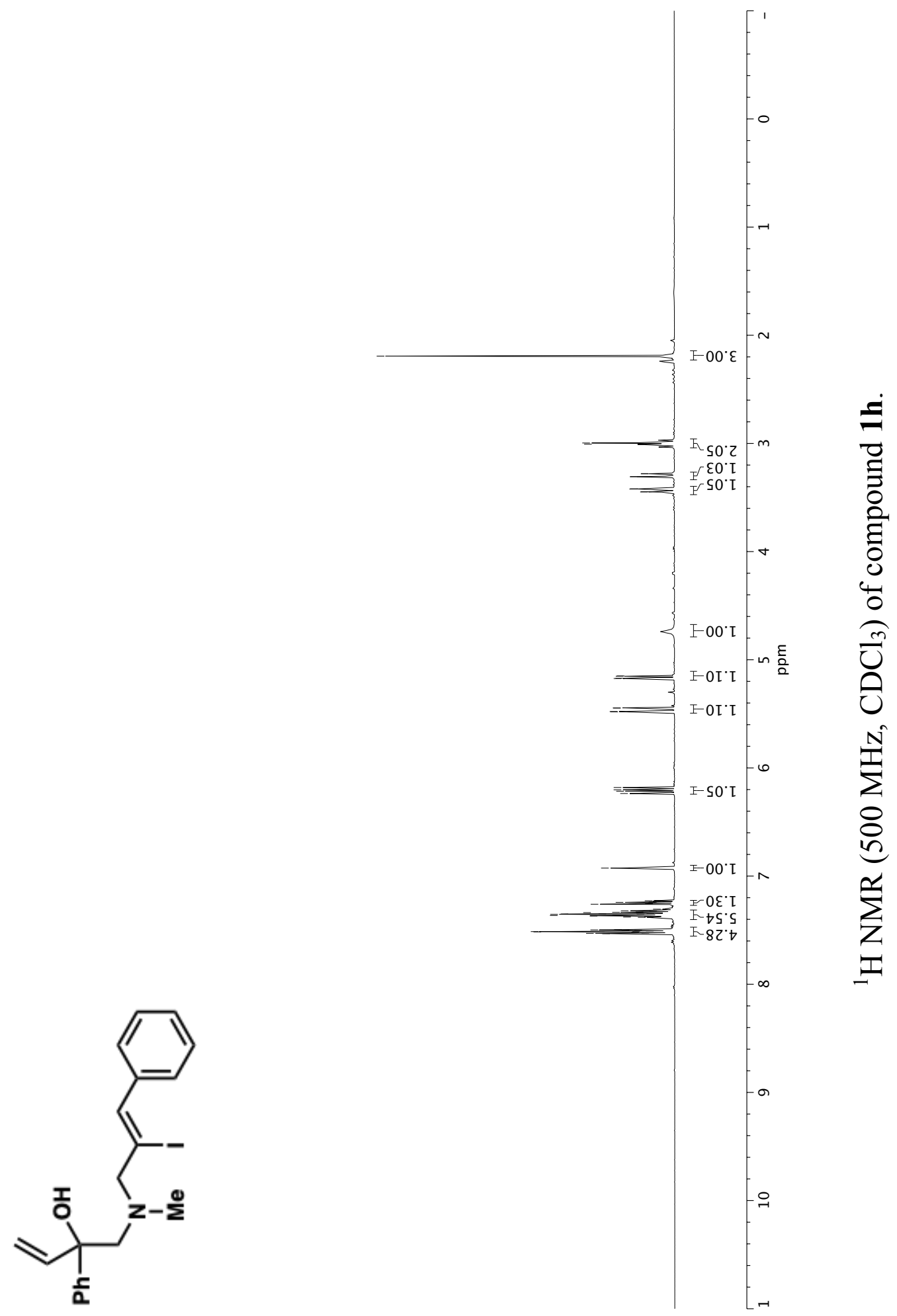




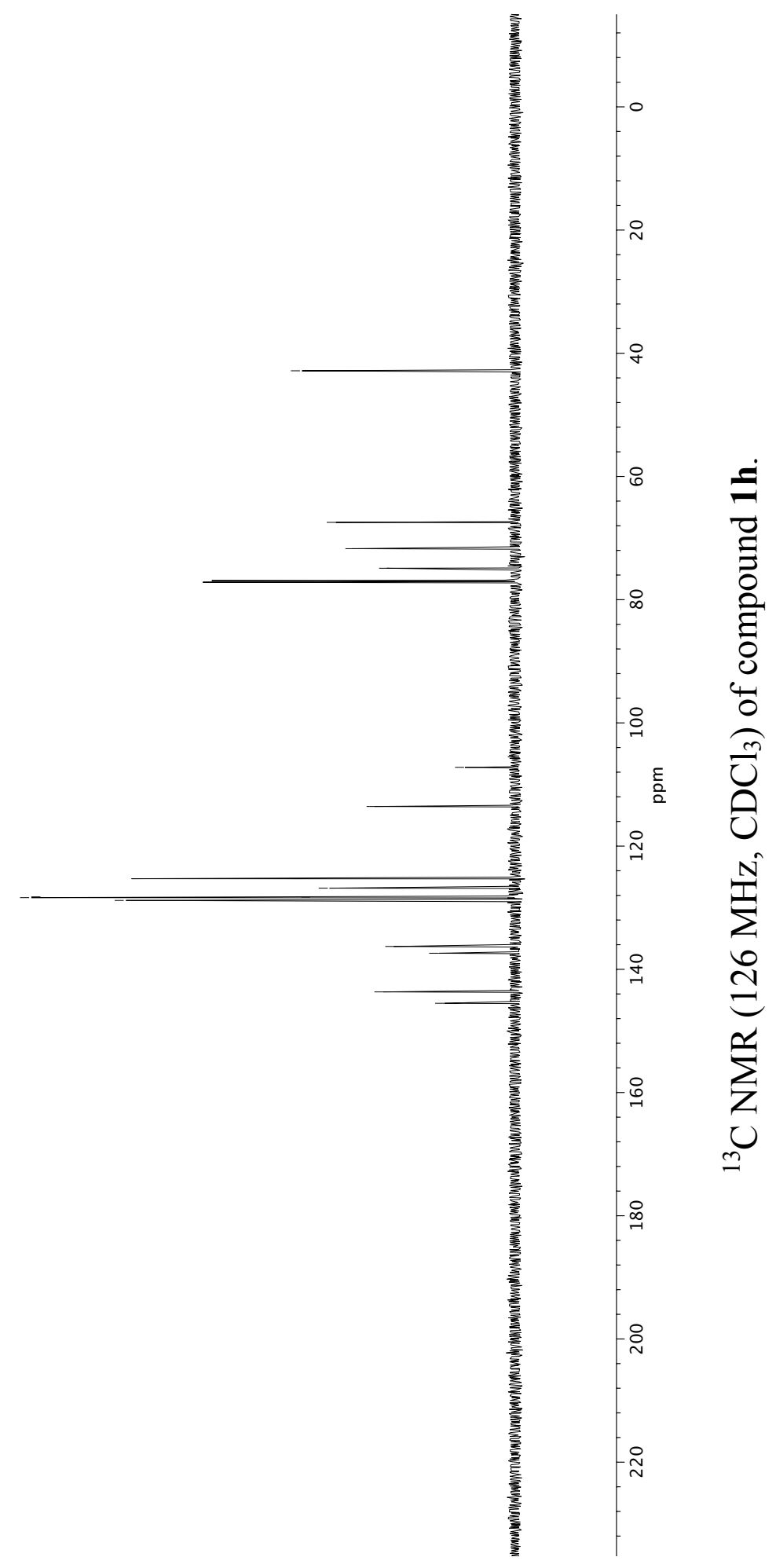

$\varepsilon .9 \varepsilon \mathrm{I}-$

$\forall \cdot \angle \varepsilon I$

$\angle \cdot \varepsilon \nabla I-$

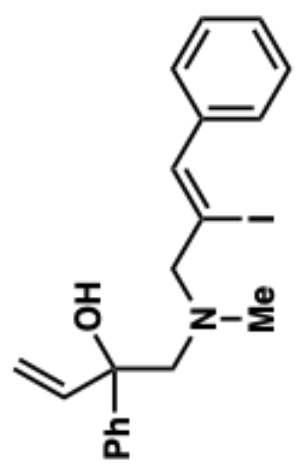




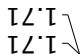

$9 \angle$ ' I

$9<\mathrm{L}]$

ऽ६ ¿-

ILZ-

$\angle \nabla^{\circ} \varepsilon-$

$0 \nabla^{\circ} \mathrm{S}$

$96^{\circ} 9$

I $\varepsilon^{\circ} L$

$\varepsilon \varepsilon^{\circ}\llcorner$

$\varepsilon \varepsilon^{*} L$

$\varepsilon \varepsilon^{\circ}\llcorner$

SE $L$ ]

$9 \varepsilon^{\circ} L$

$9 \varepsilon^{\circ} L-$

$9 \varepsilon^{\circ} L$

$8 \varepsilon^{\circ} L$

IS $L^{-}$

ZS' $\angle$

2S 2

ES $L$

\&S. $L-$

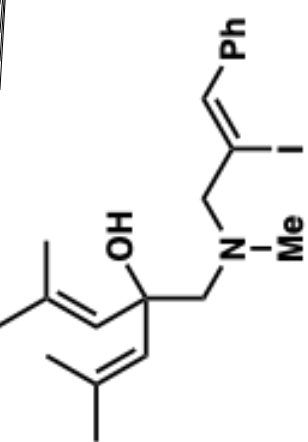

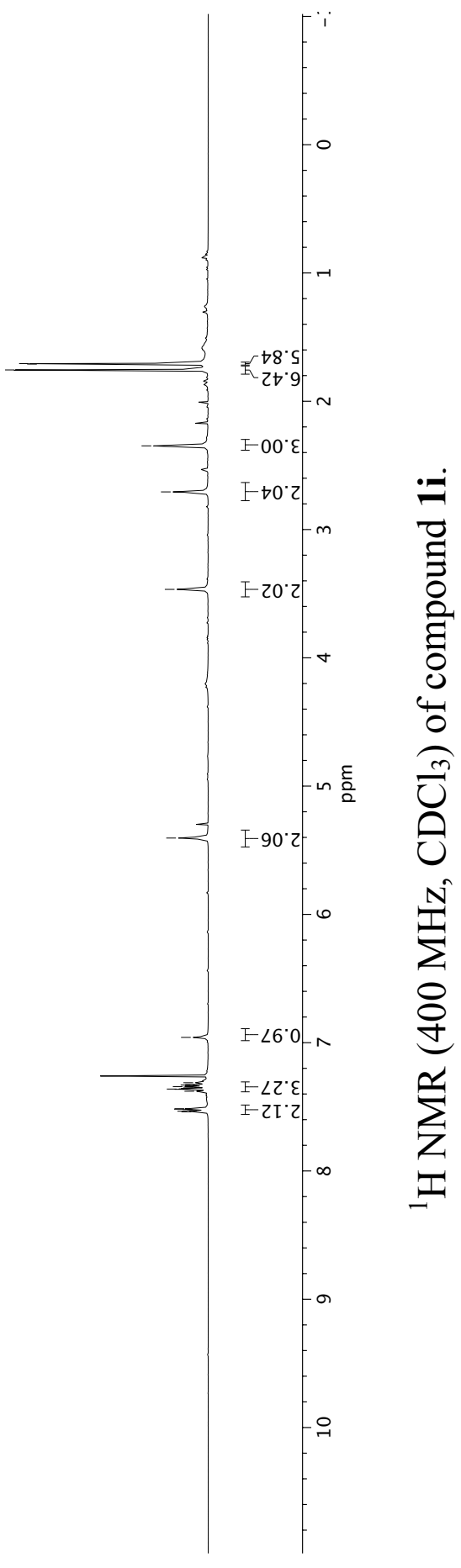



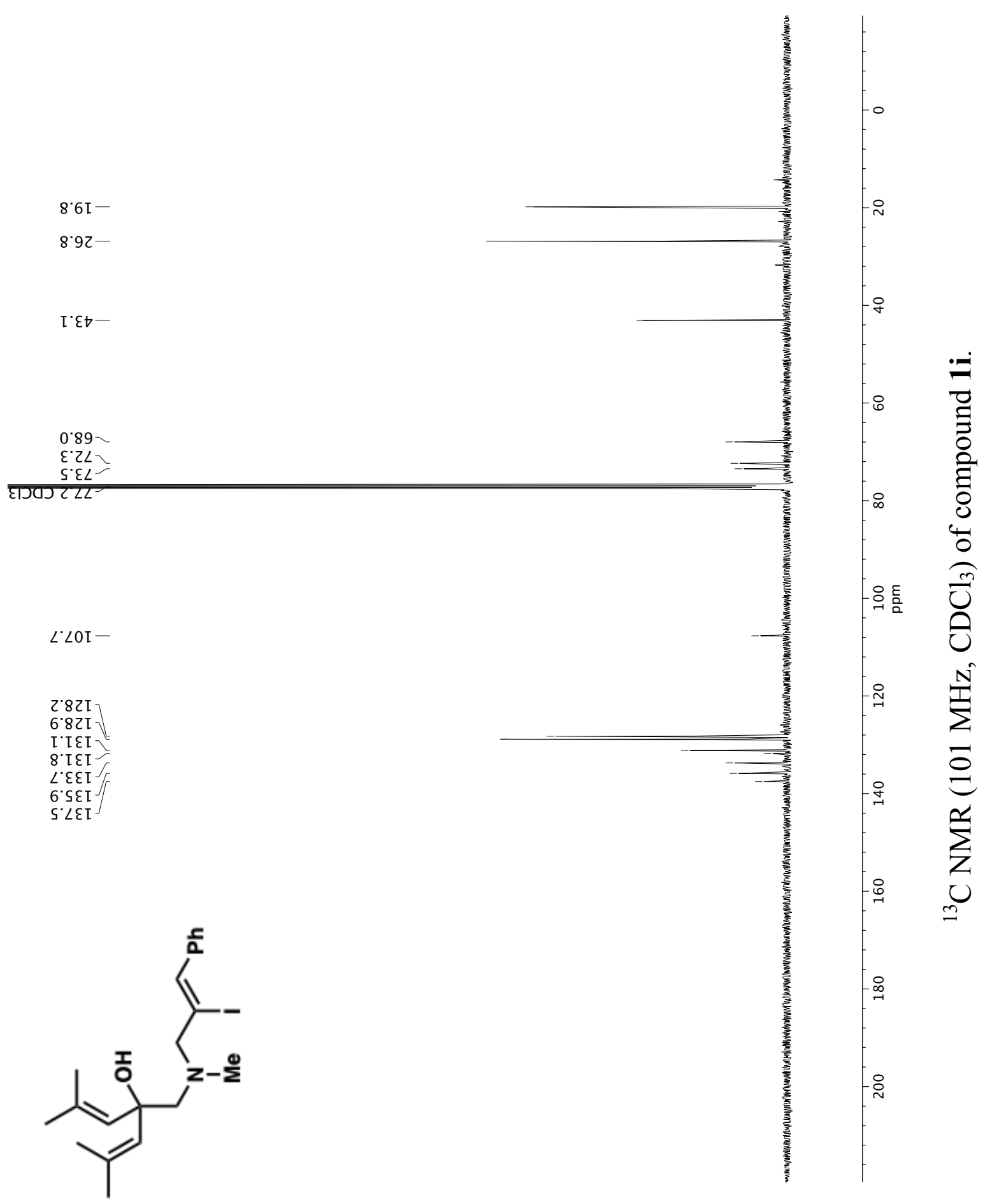
$08 \cdot 2-$

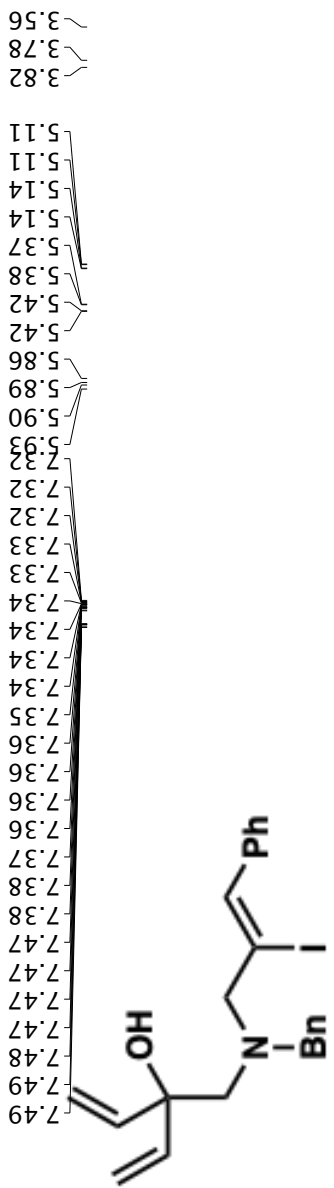

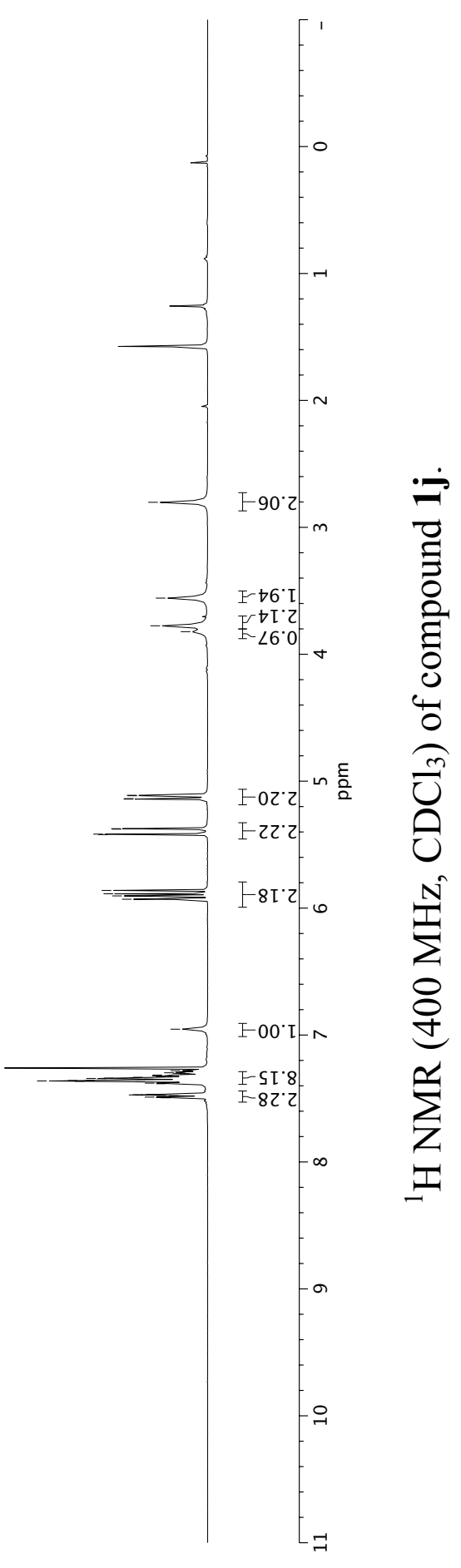




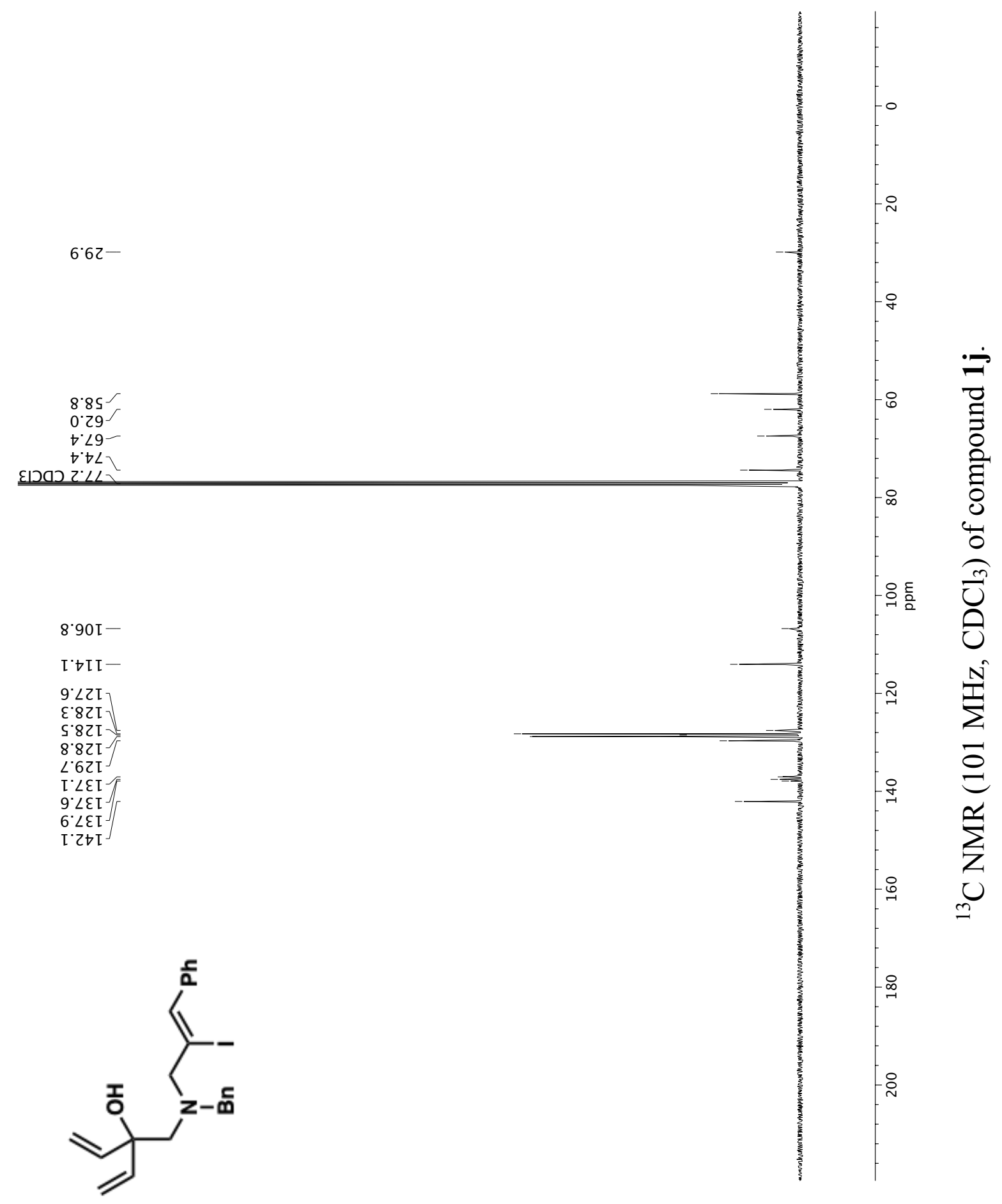


$\mathrm{I} L^{\circ} \mathrm{I}-$

$82^{\prime} 2-$

$96 \cdot 2-$

$2 \varpi^{*} \varepsilon-$

$\varepsilon 9^{\circ} \sigma^{-}$

$\angle 6^{\circ} \mathrm{b}$

ZI'S -

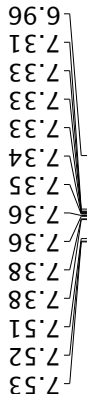

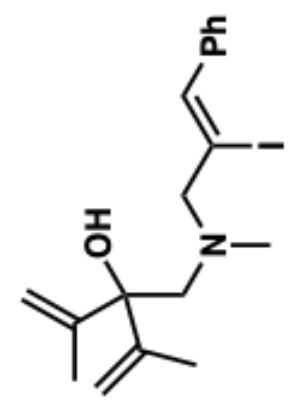

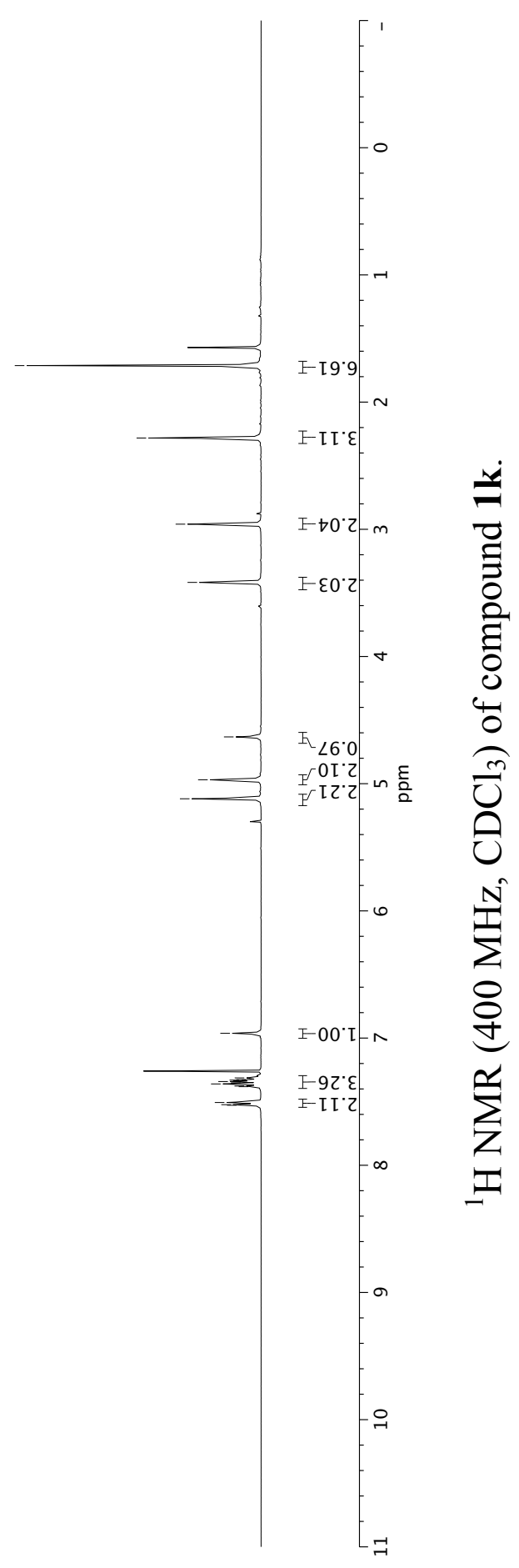


$\tau^{\prime} \angle t I-$

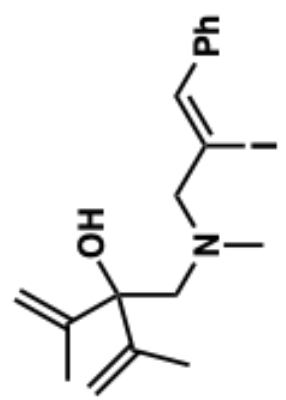




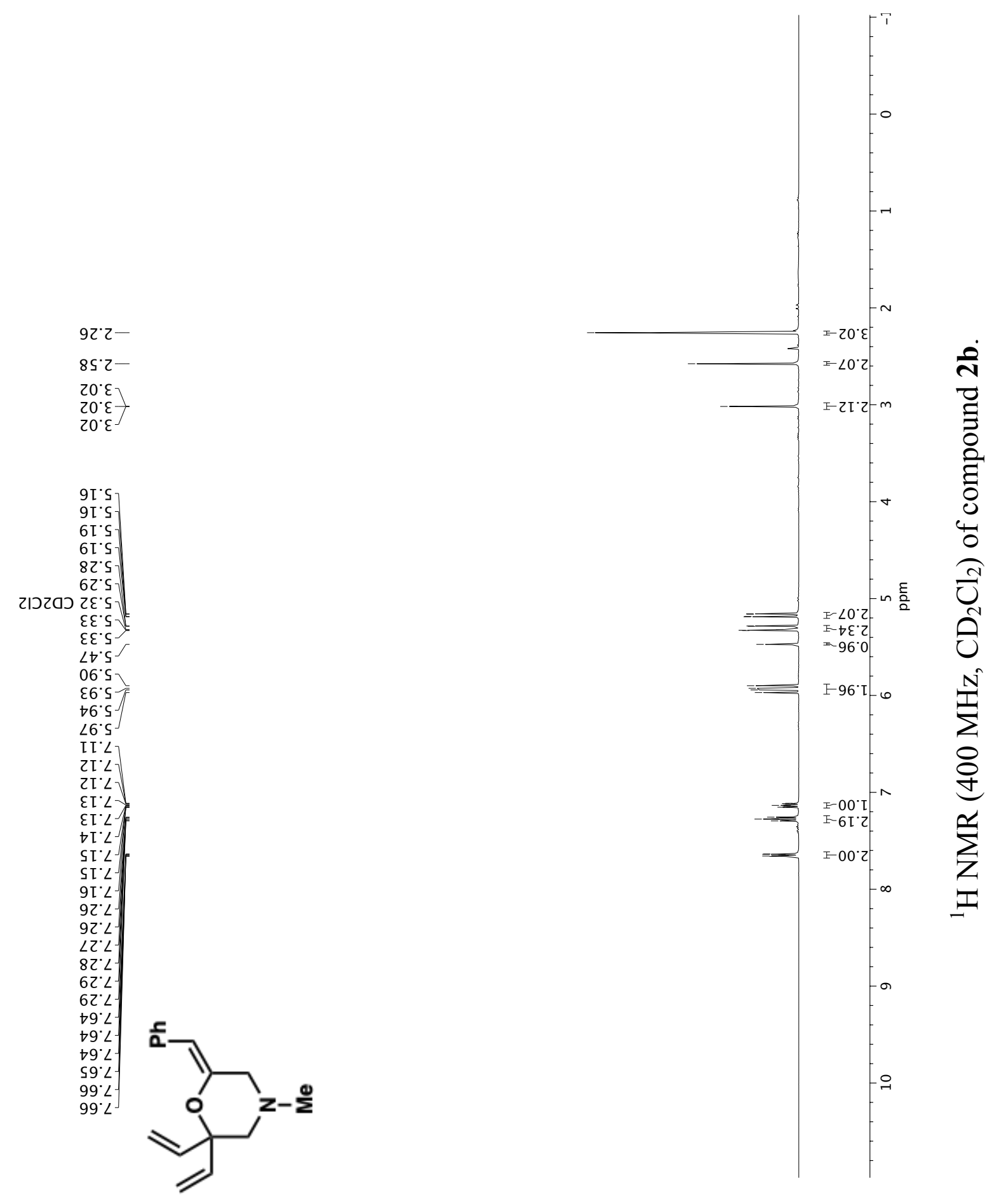




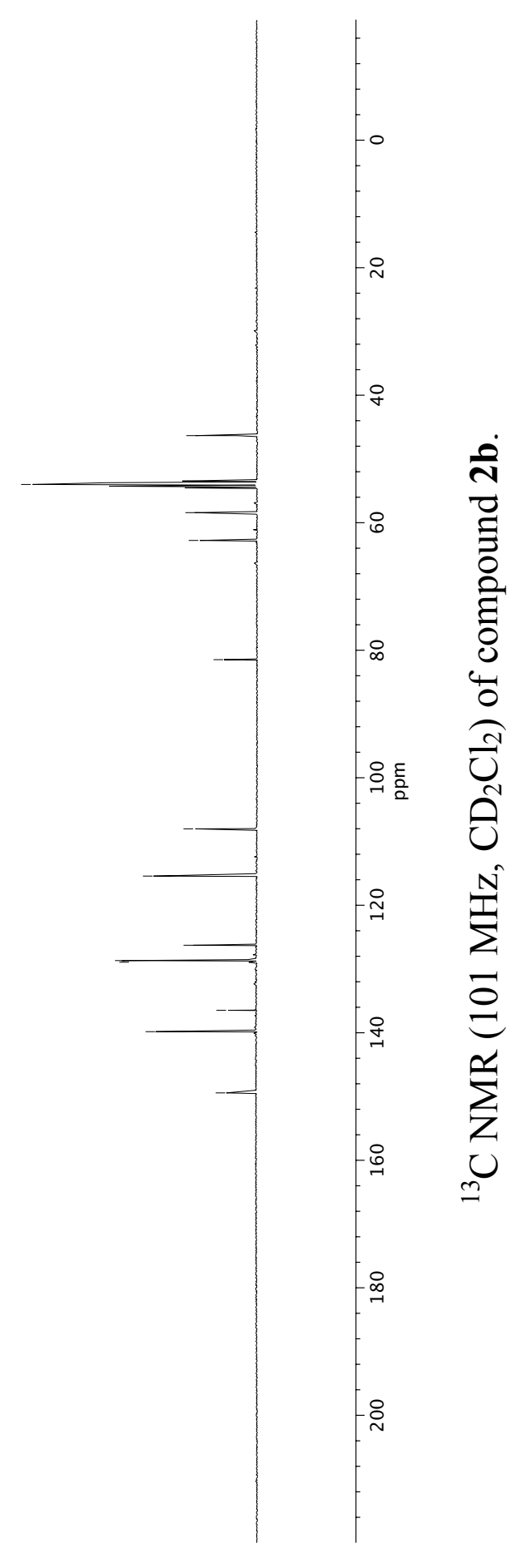

0.80 I -

t.SIL-

$\varepsilon^{\circ} 92 \mathrm{I}$

$\angle 82 I$ $6.82 \mathrm{I}$

ᄃ.9EI--

$8^{\circ} 6 \varepsilon \mathrm{I}$

เซ 6 t I -

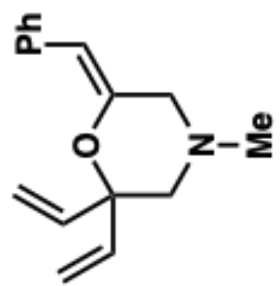

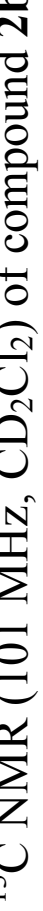



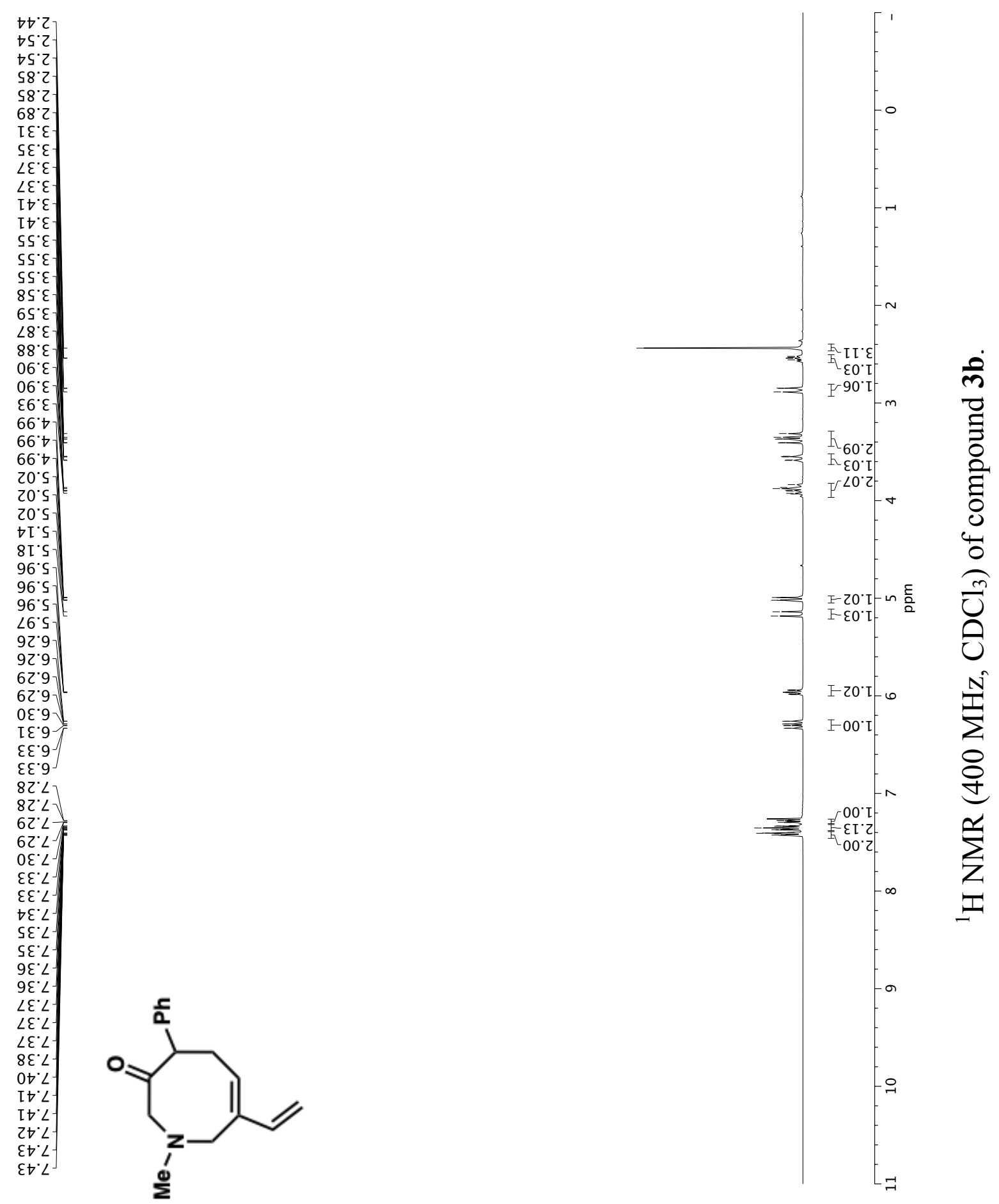


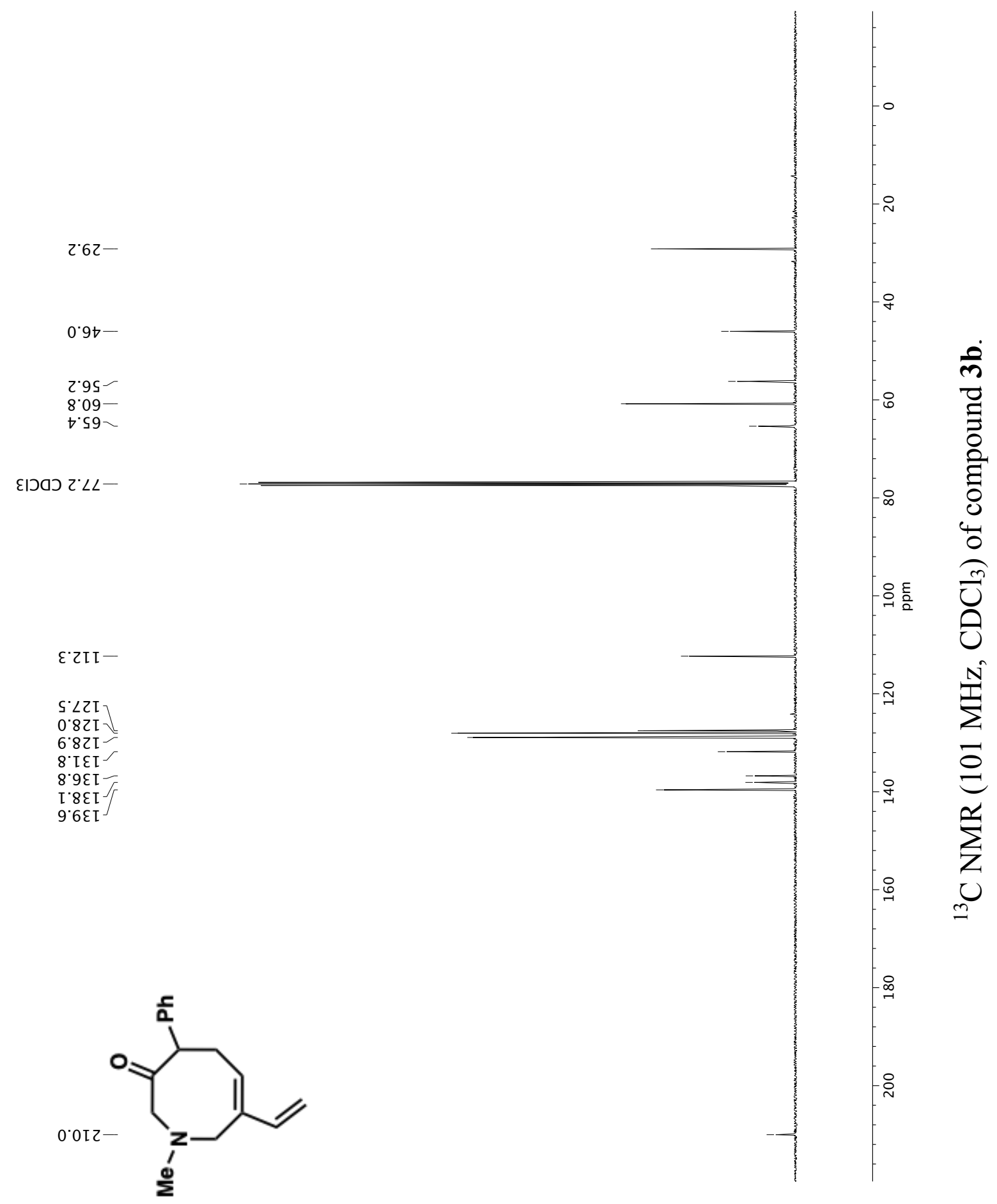



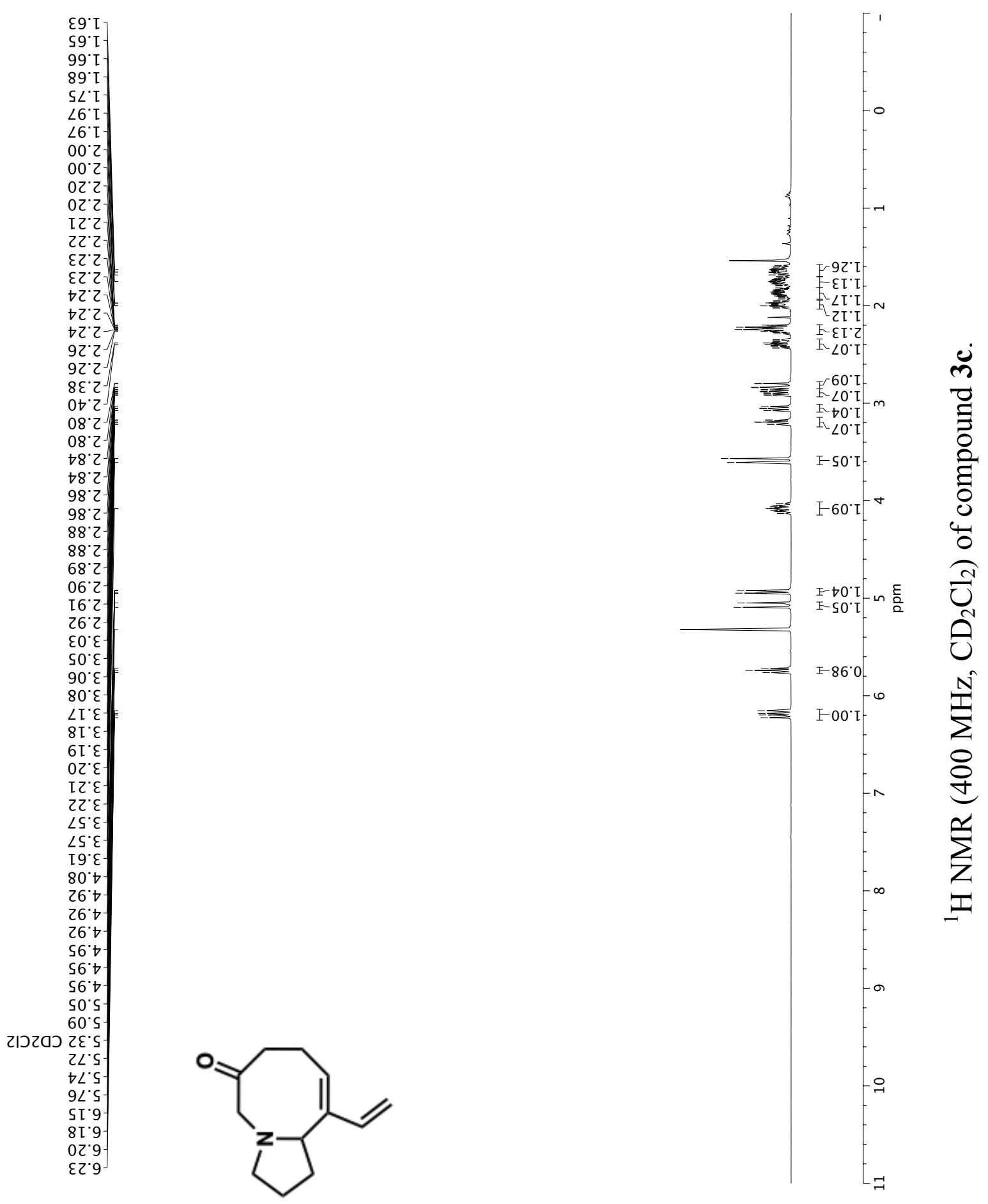


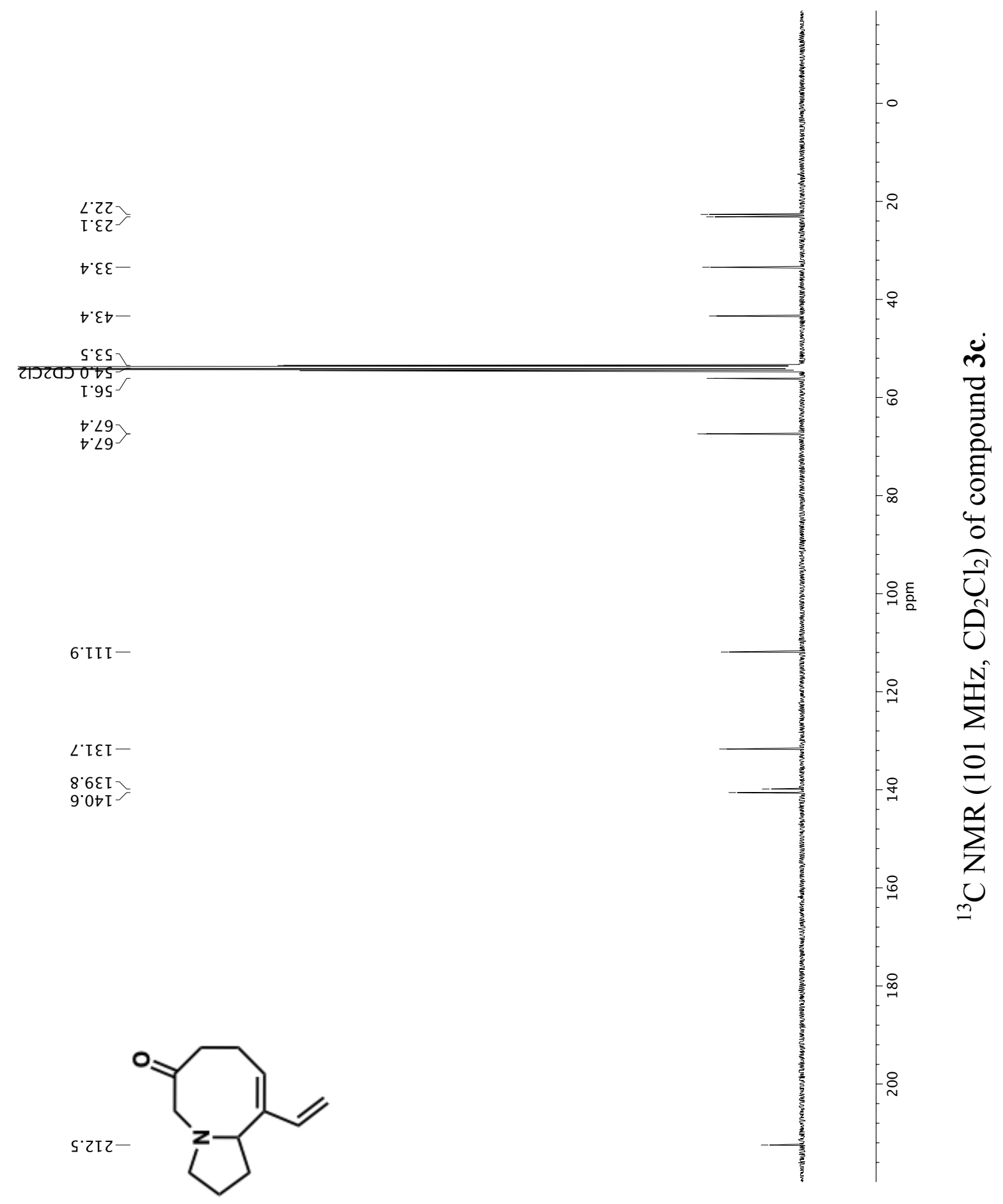



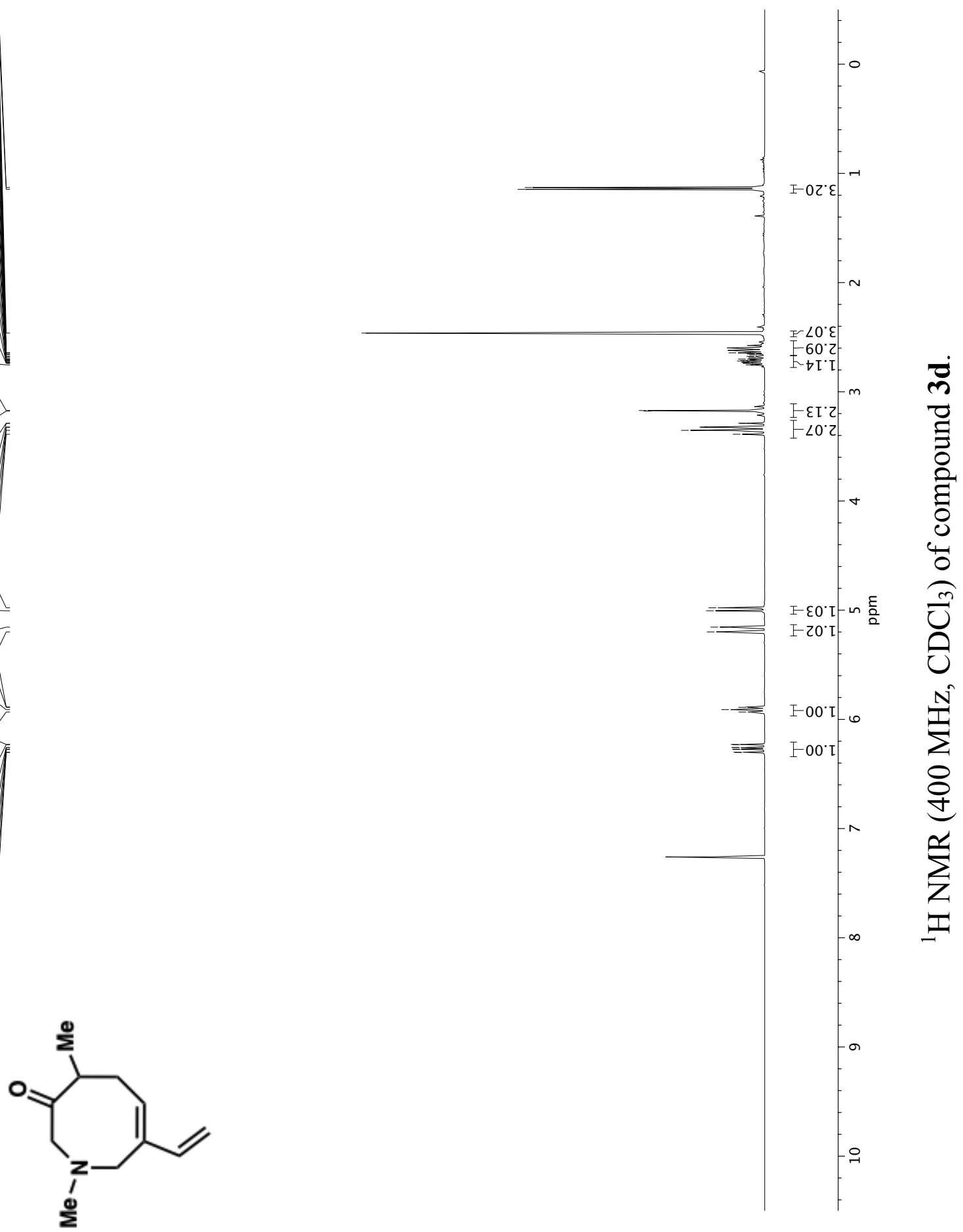


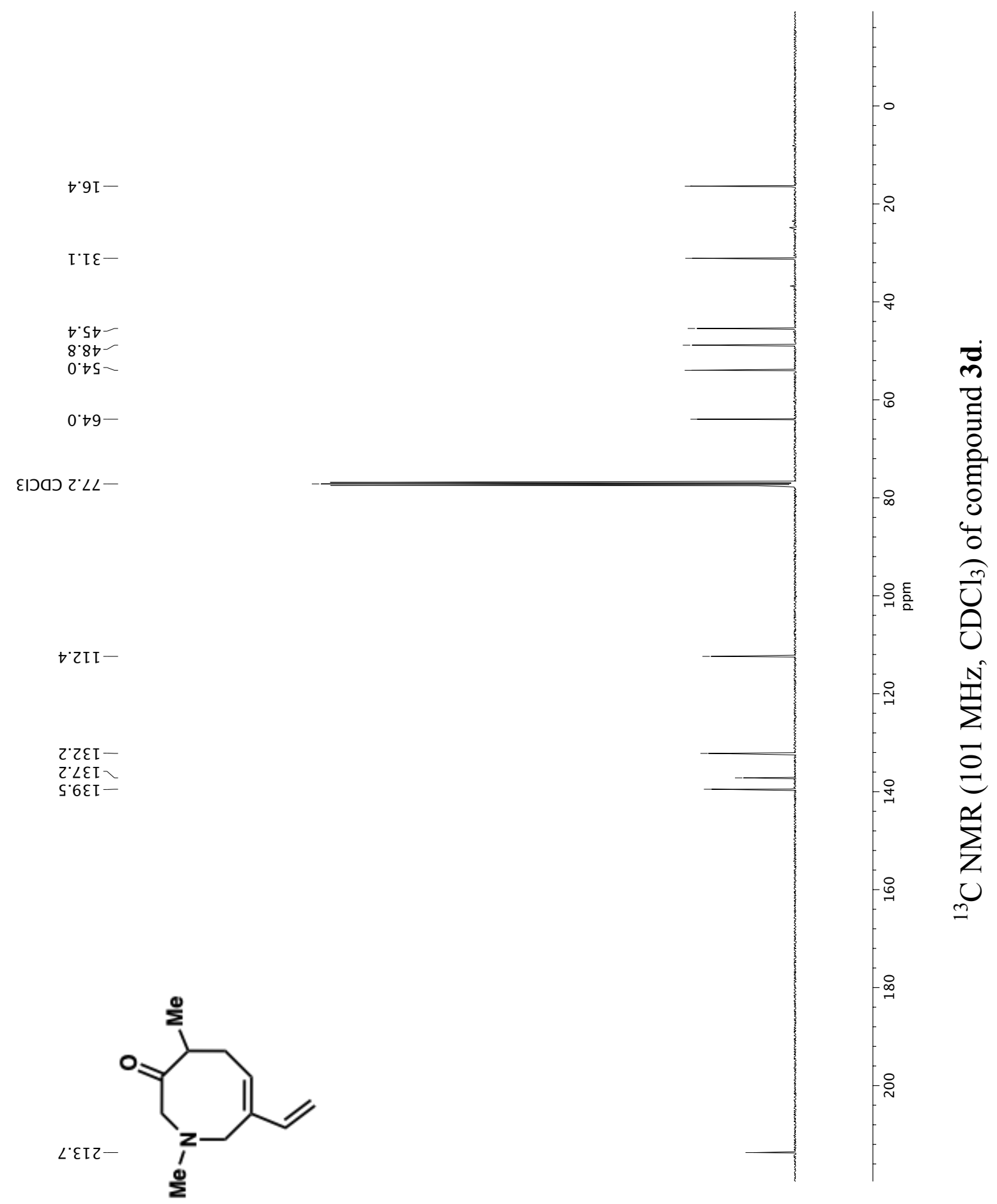




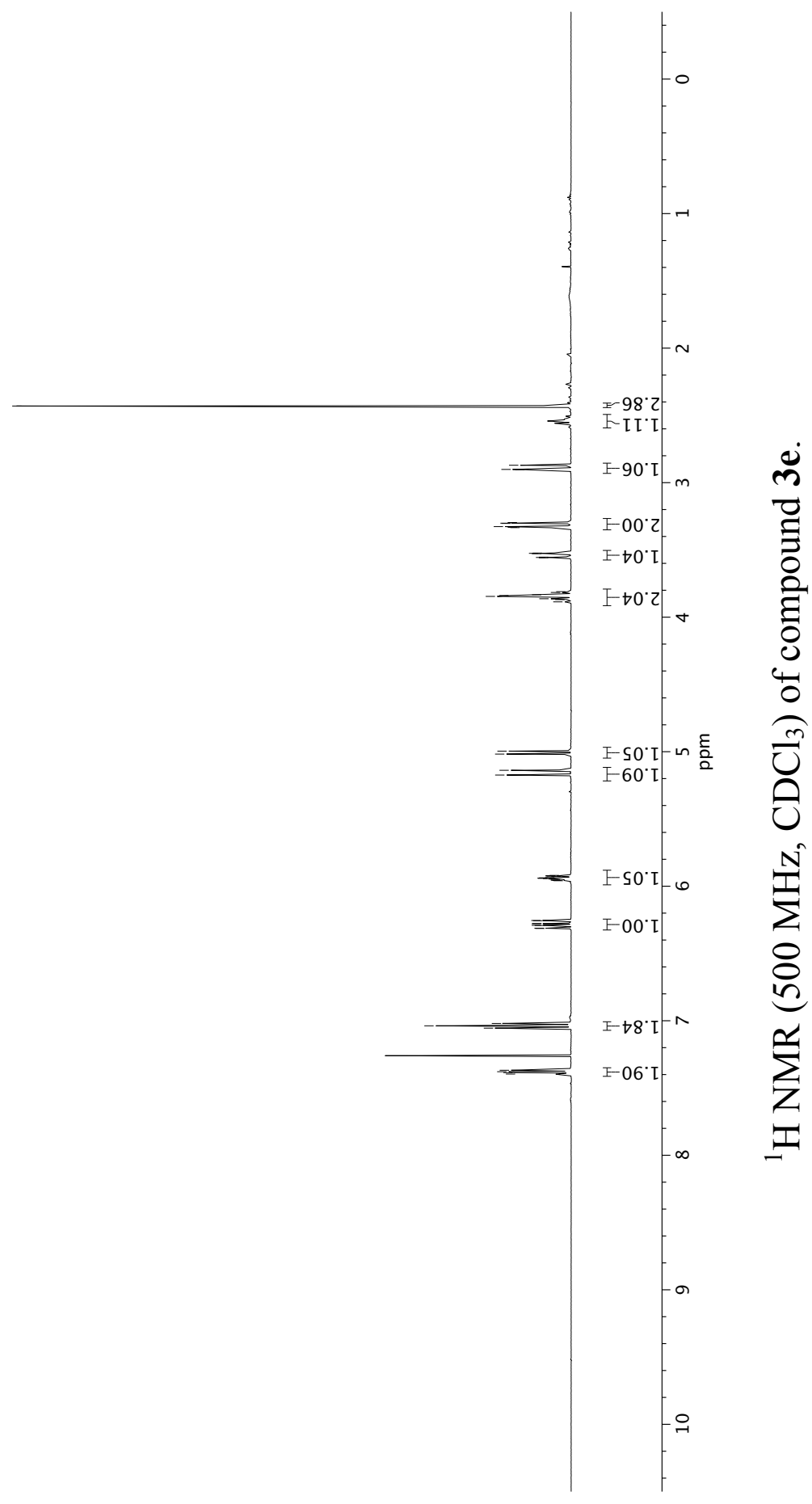


s.62-

$2 \cdot 9 t-$

5.95-

$6.6 \mathrm{~s}-$

9. $99-$

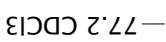

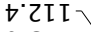

9 SI I

8 S S I

$9.62 \mathrm{~L}$

$\angle \cdot 62 I$

$\forall \cdot I \varepsilon I-$

$0 \forall \varepsilon[-$

$0 \quad t \varepsilon[-$

$6^{\circ} 9 \varepsilon \mathrm{I}$
$\mathrm{s}^{\circ} 6 \varepsilon \mathrm{I}$

Z'โ9I

$\tau^{\prime} \varepsilon 9 I^{-}$

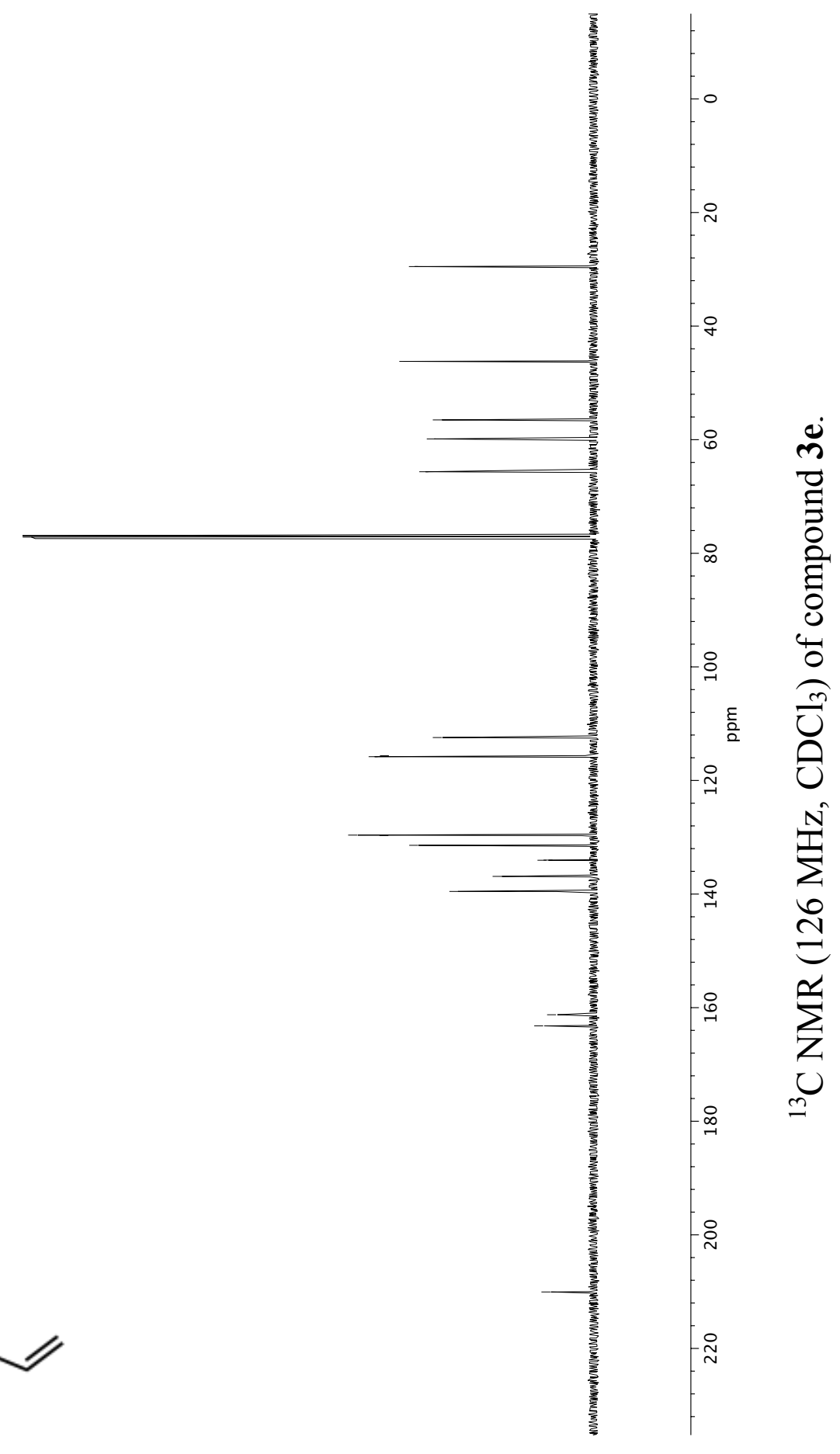




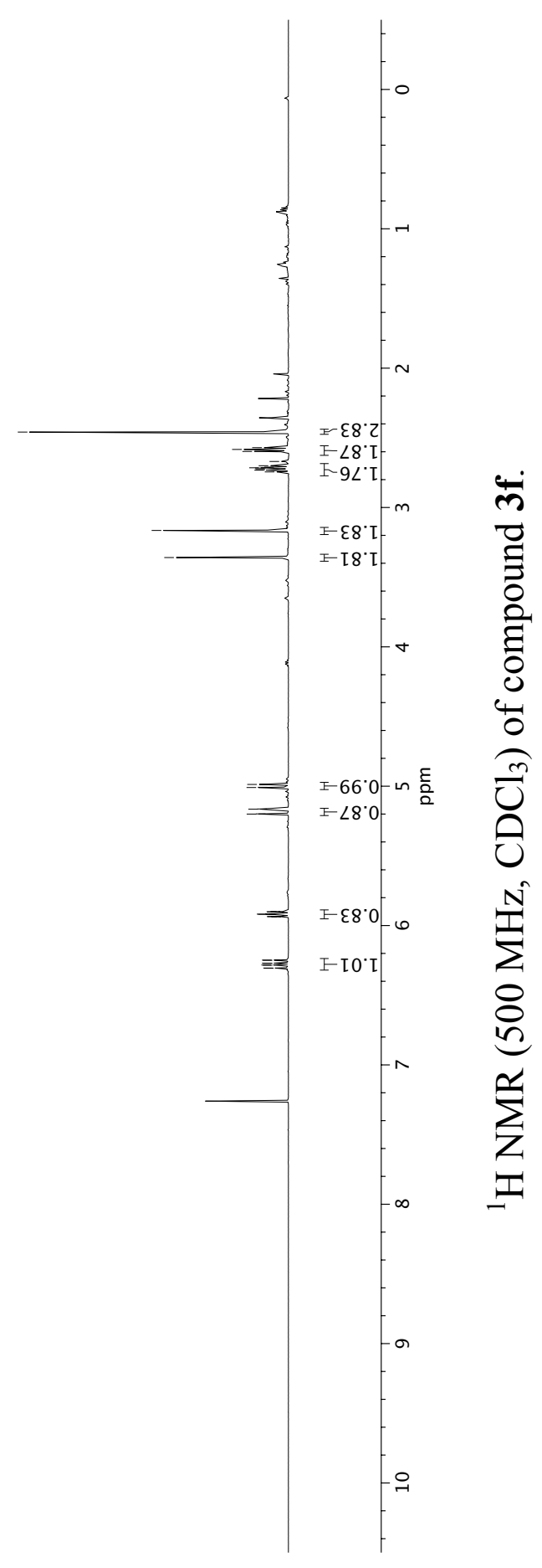




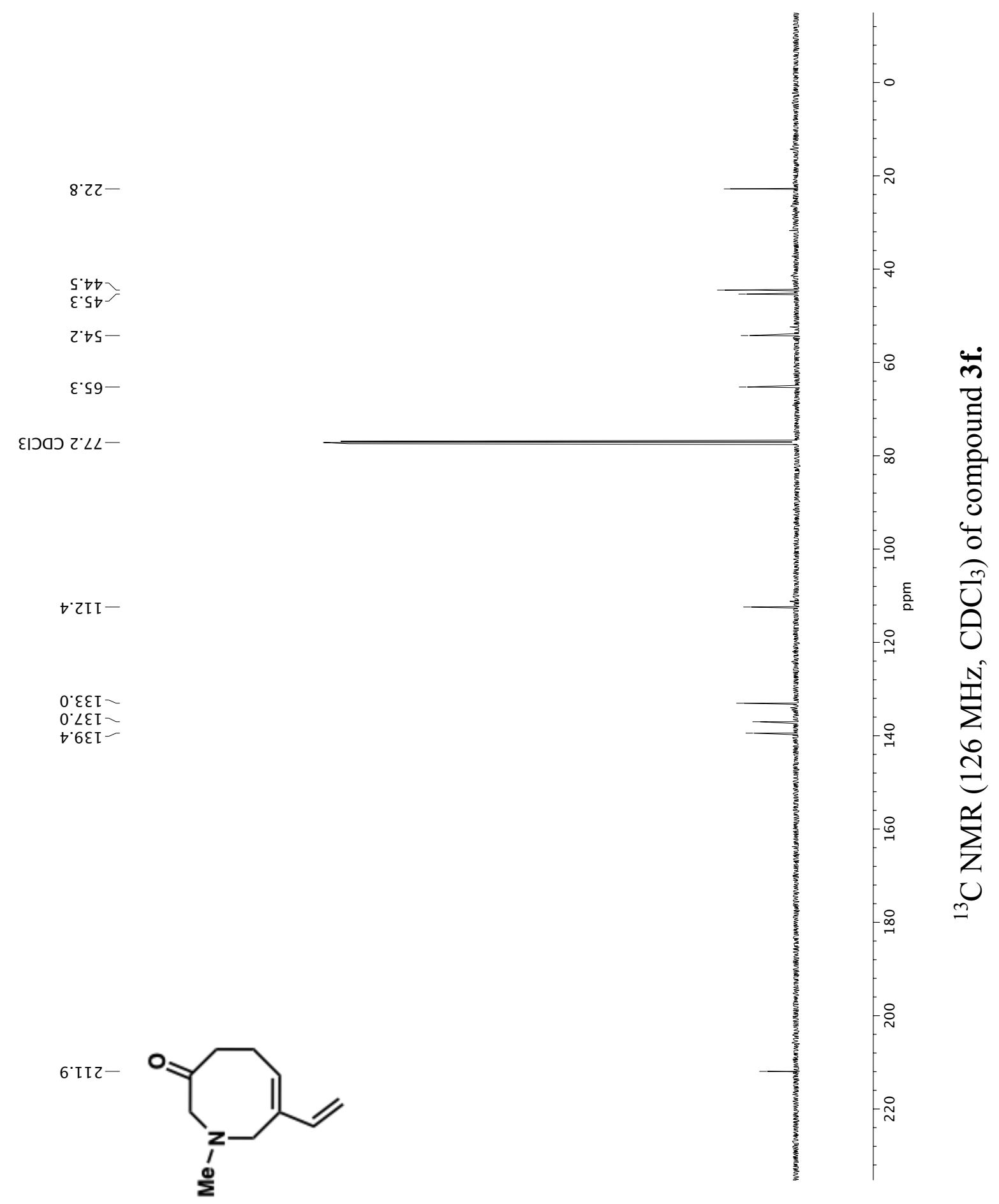




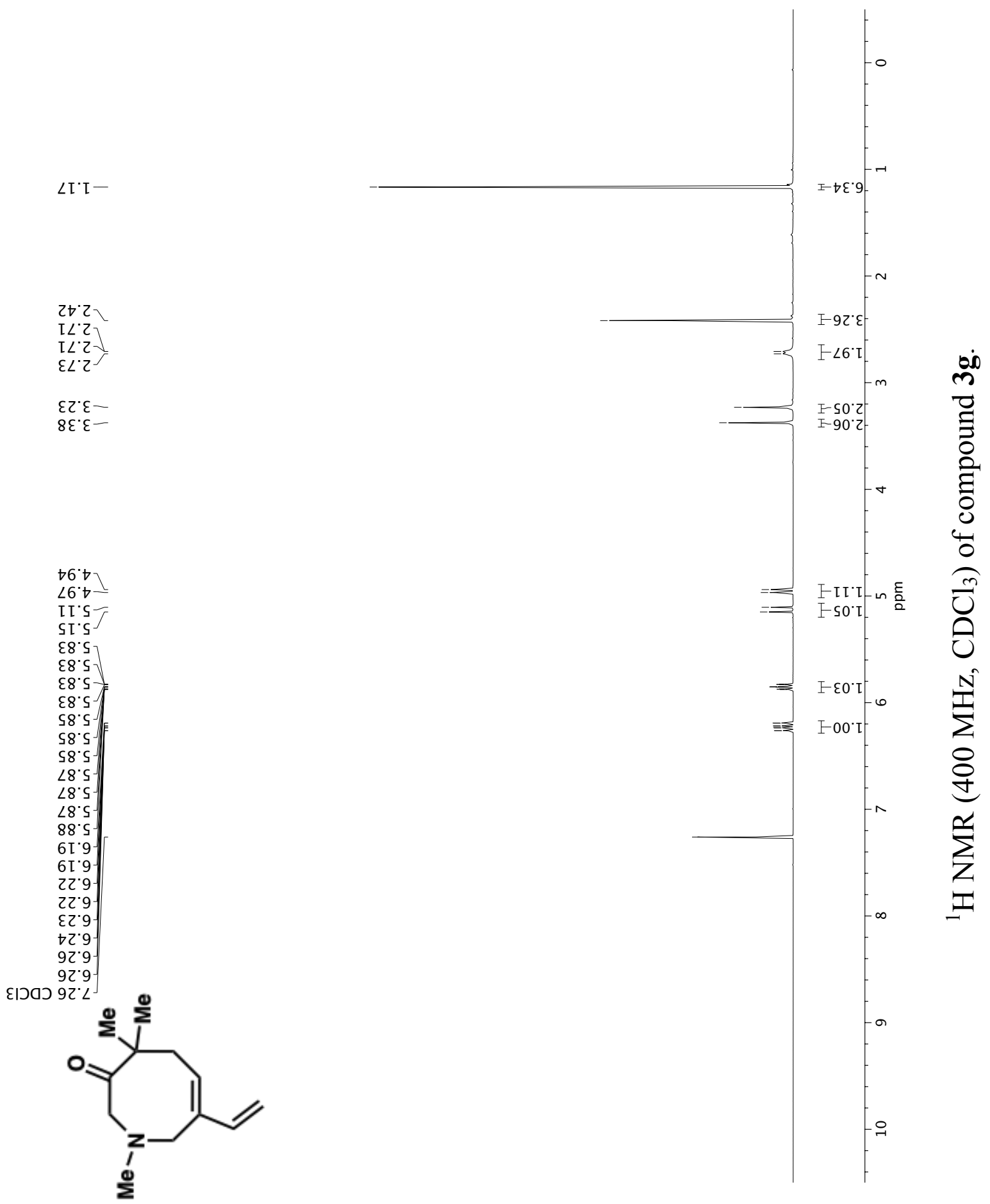




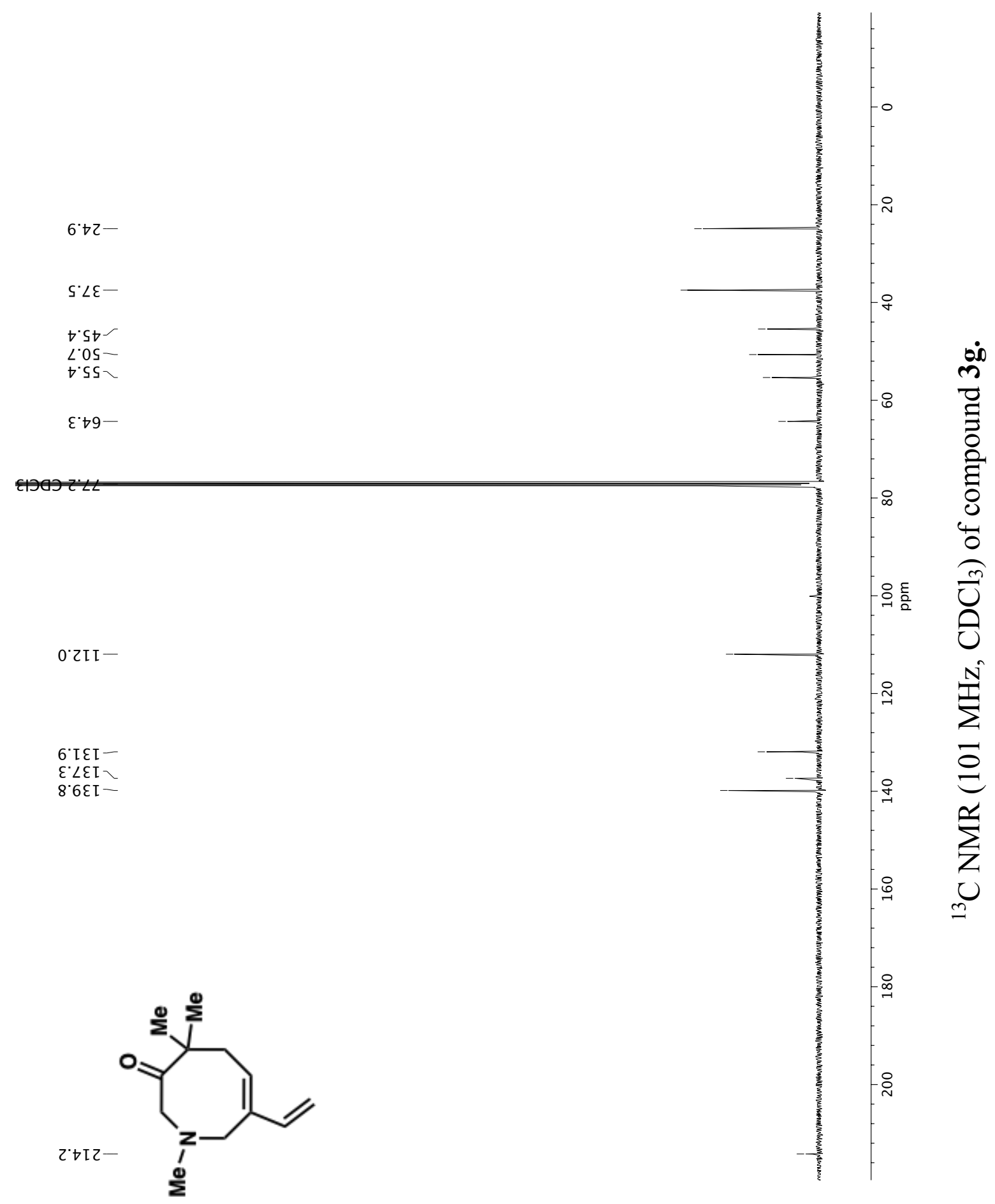



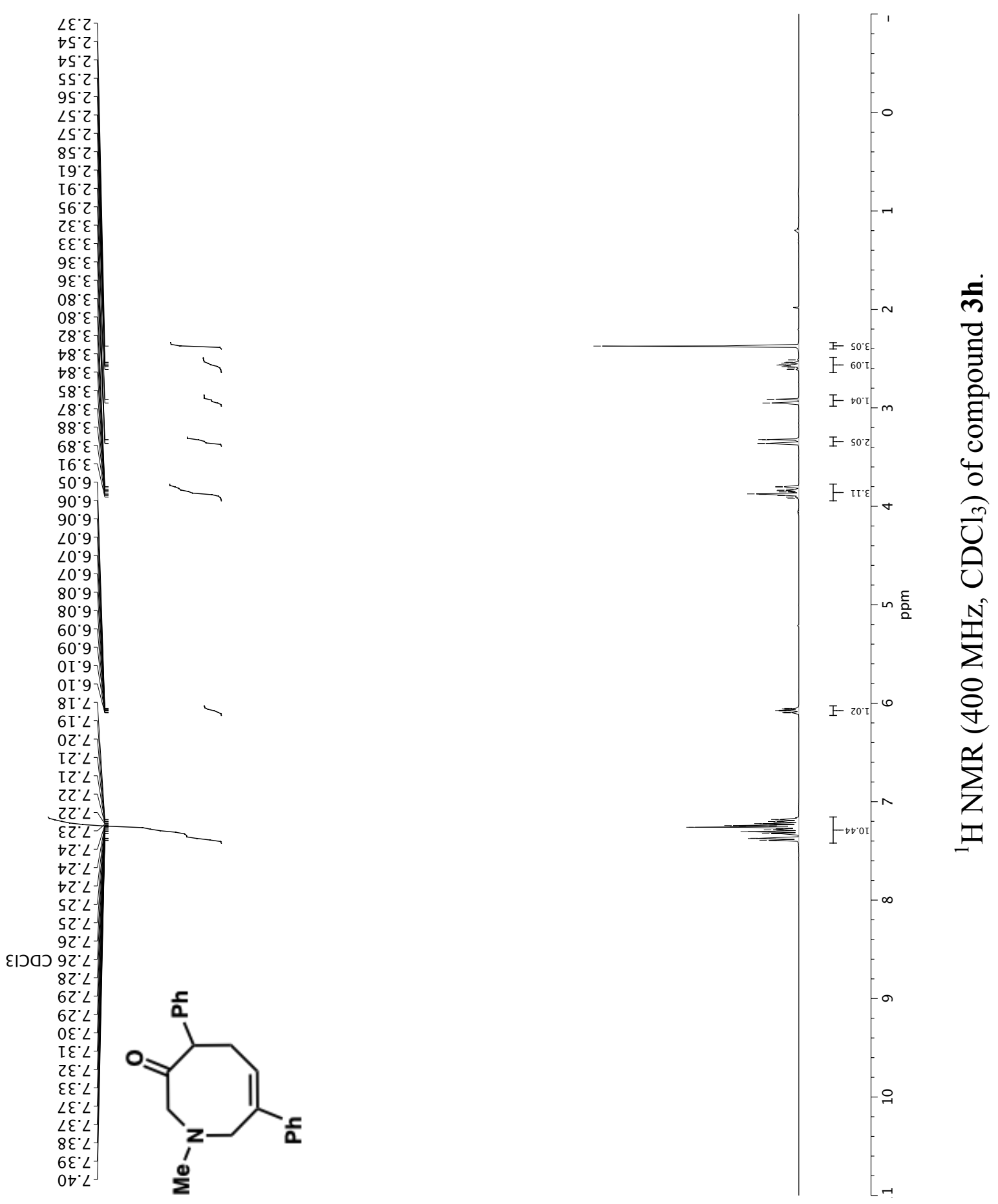


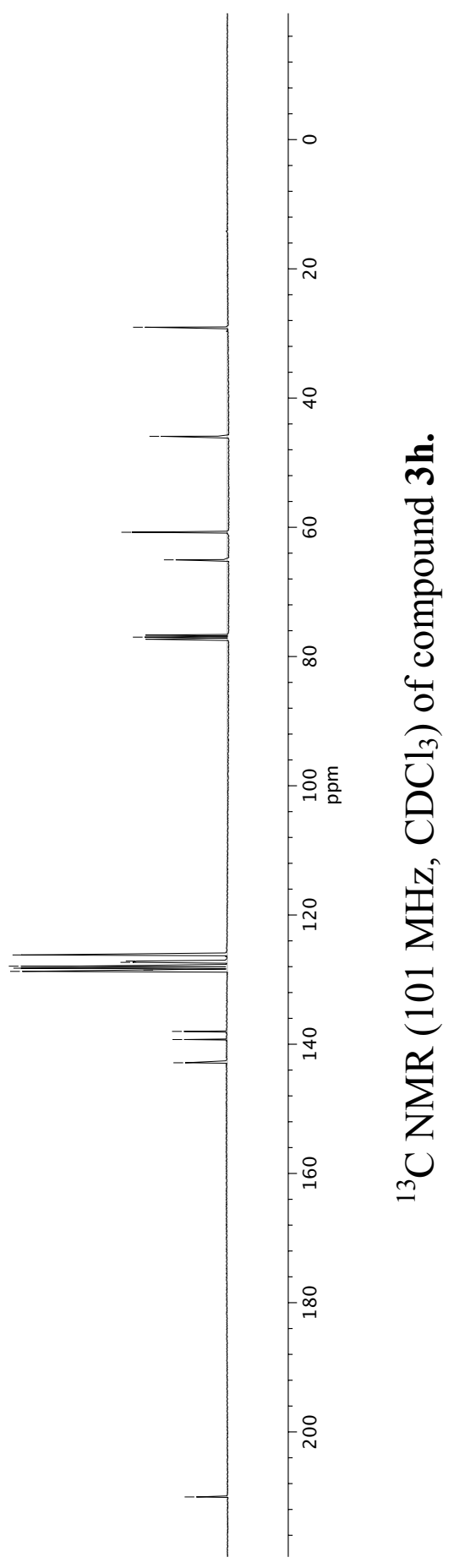


$\triangle \angle 0^{\circ} 0-$

†0 $\mathrm{I}-$

โ8. $\mathrm{I}$

$60^{\circ} 2$

$2 I^{\circ} Z$
St'
$89^{\prime} 2$

$89^{\circ} 2$

$69^{\circ} \mathrm{Z}$

[ $\angle{ }^{\prime} \mathrm{Z}$ ]

2
2

$80^{\circ} \varepsilon$
$60^{\circ} \varepsilon$
$2 L^{\prime} \varepsilon$

$\mathrm{ZI} \cdot \varepsilon$

ZI. $\varepsilon$

$2 \nabla^{\circ} \varepsilon$

$S t^{\circ} \varepsilon$

s9.t

โ6 ${ }^{\circ}$

$26^{\circ} 5^{-}$

$68^{\circ} \mathrm{s}-$

$\begin{array}{ll}22 & 2 \\ \varepsilon Z^{\circ}< \\ \varepsilon 2\end{array}$

$\varepsilon Z^{\circ} L-$

$S Z^{\circ} L$

OS' $\angle$

IS $L$ -

$25^{\circ} \mathrm{L}$

25.2

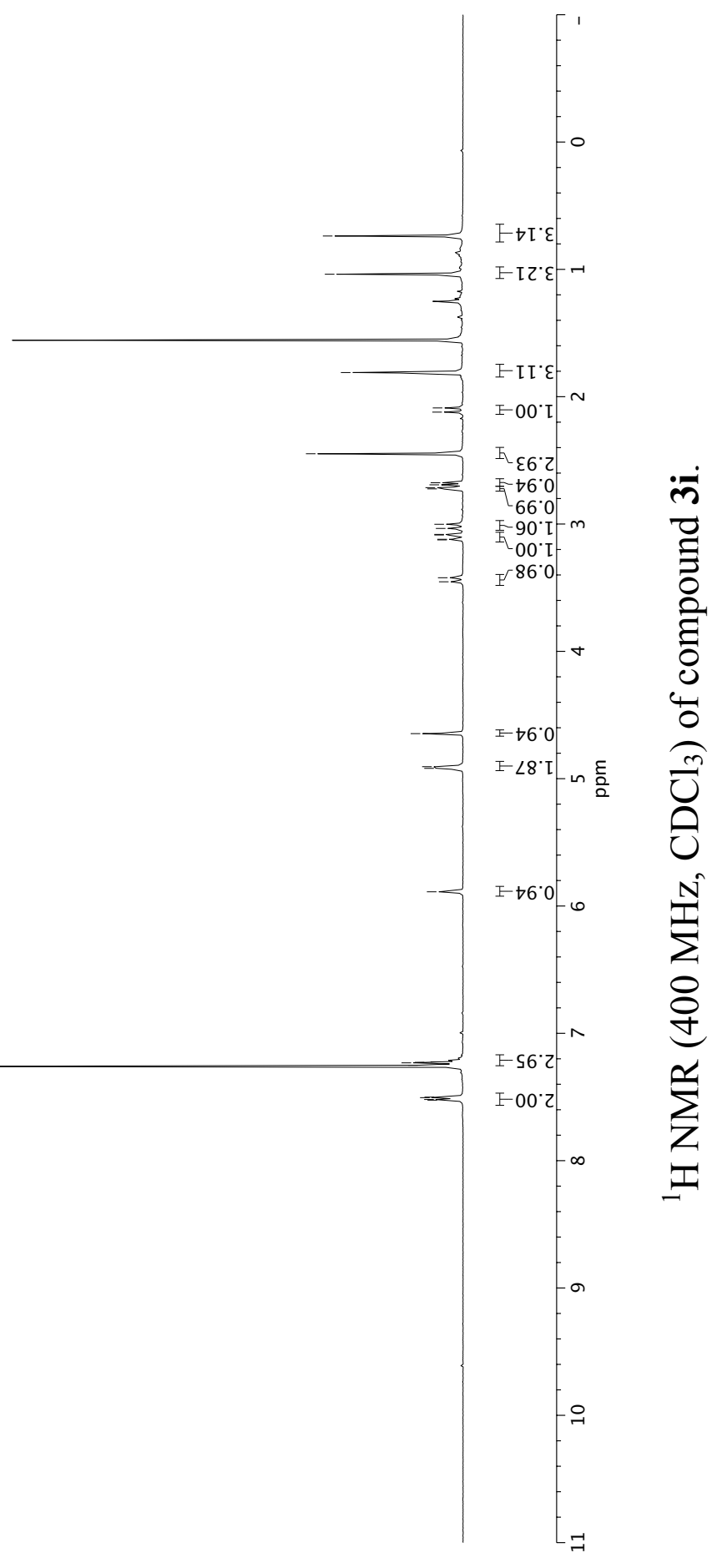



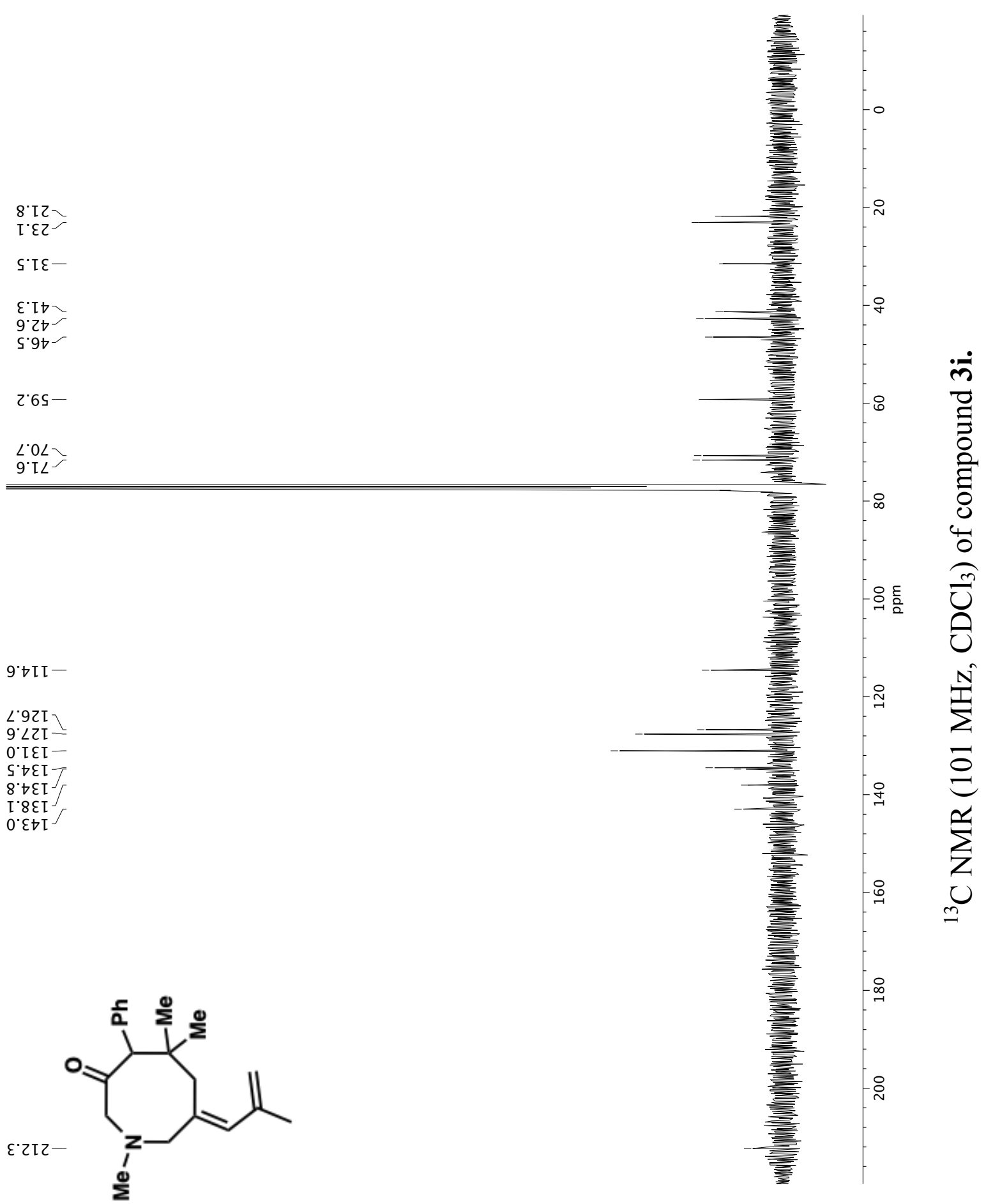

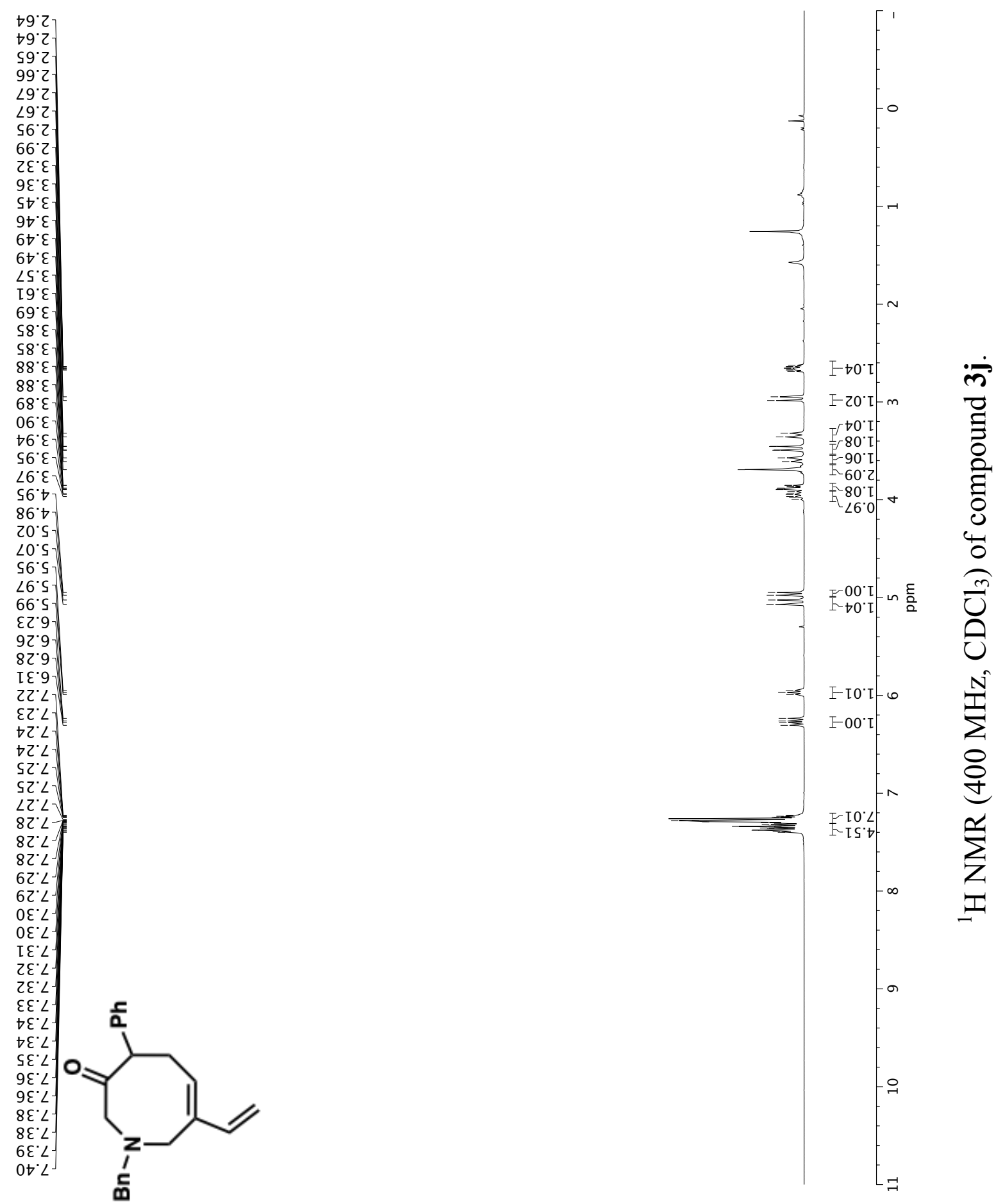


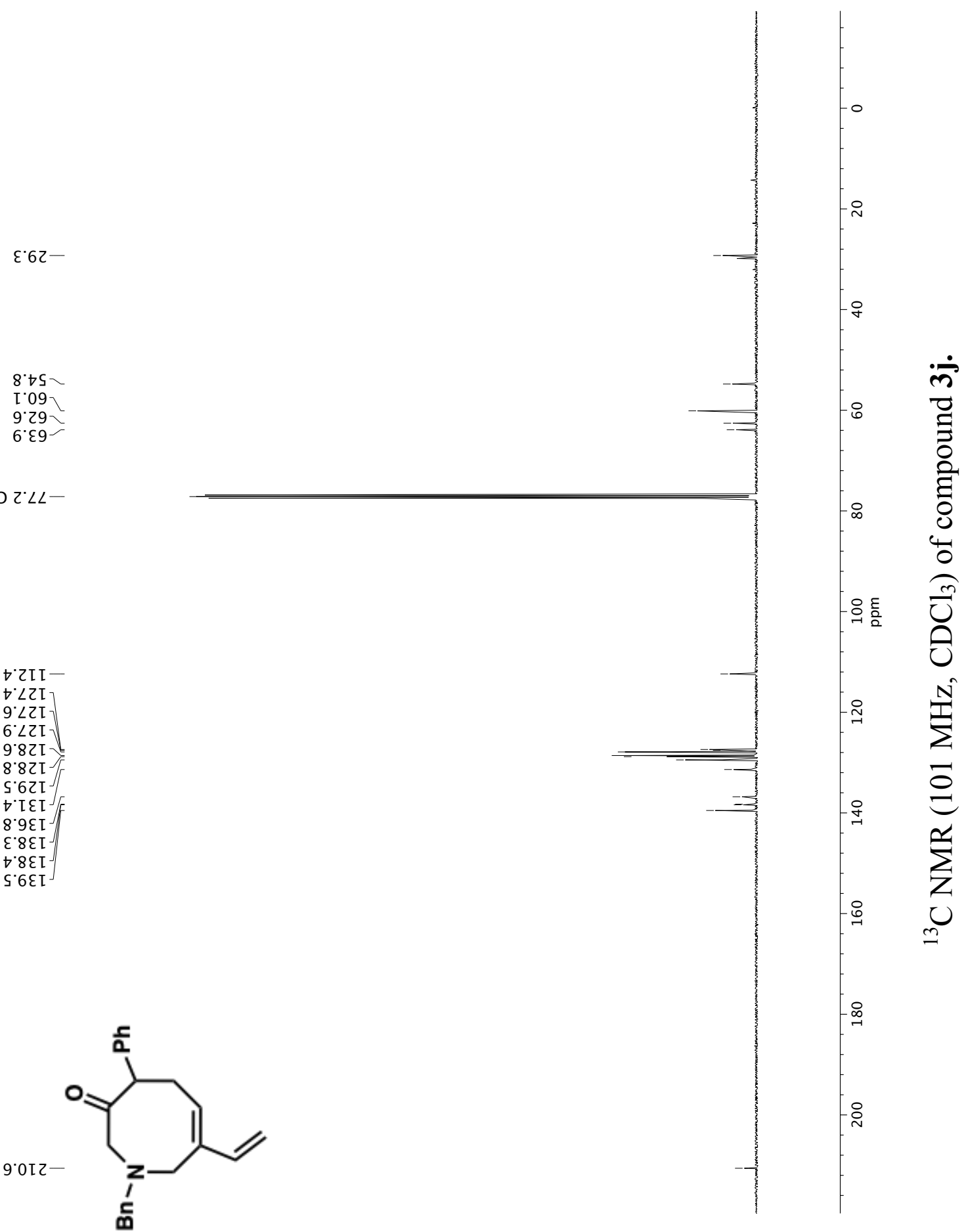



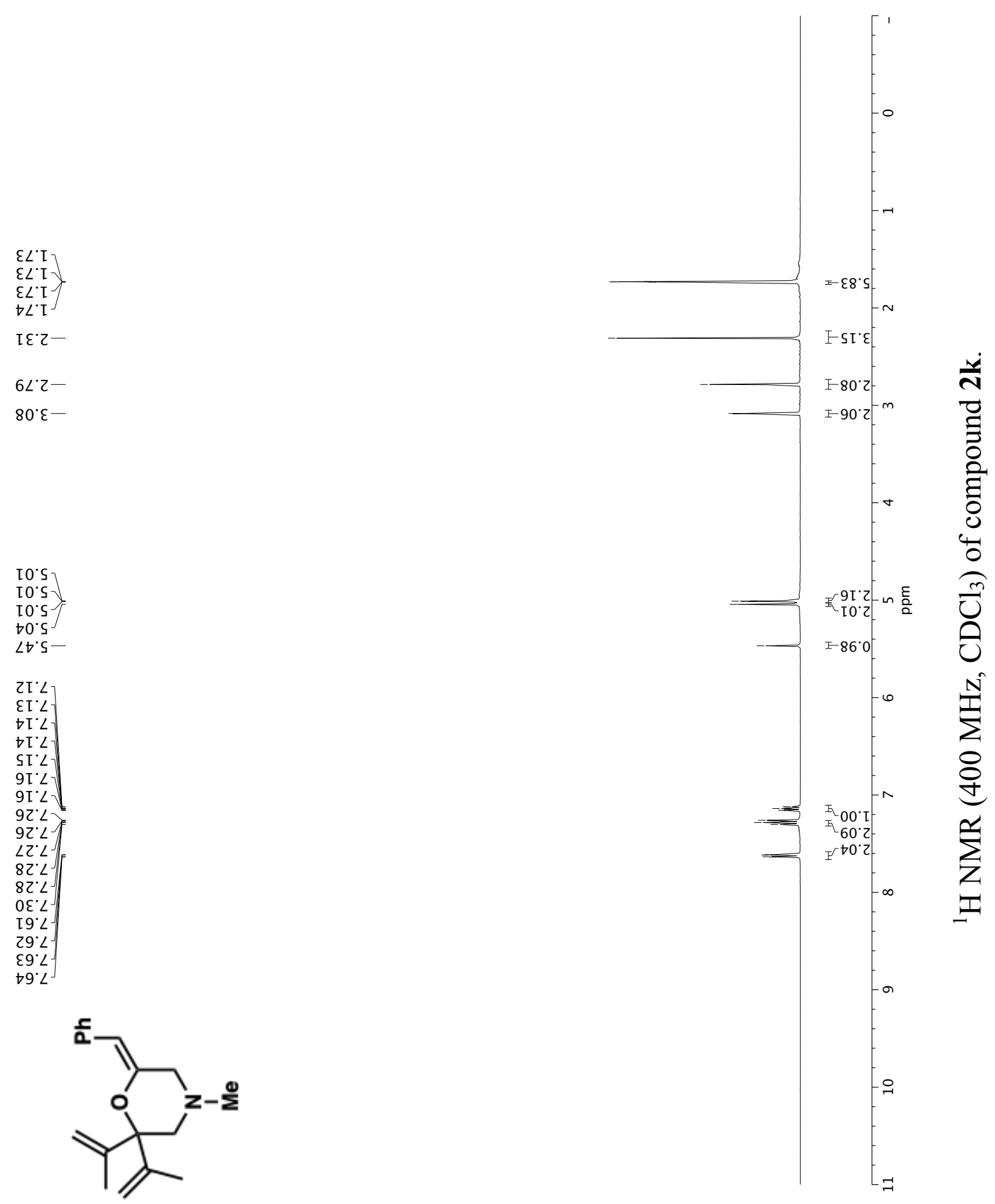

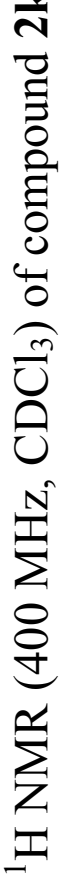




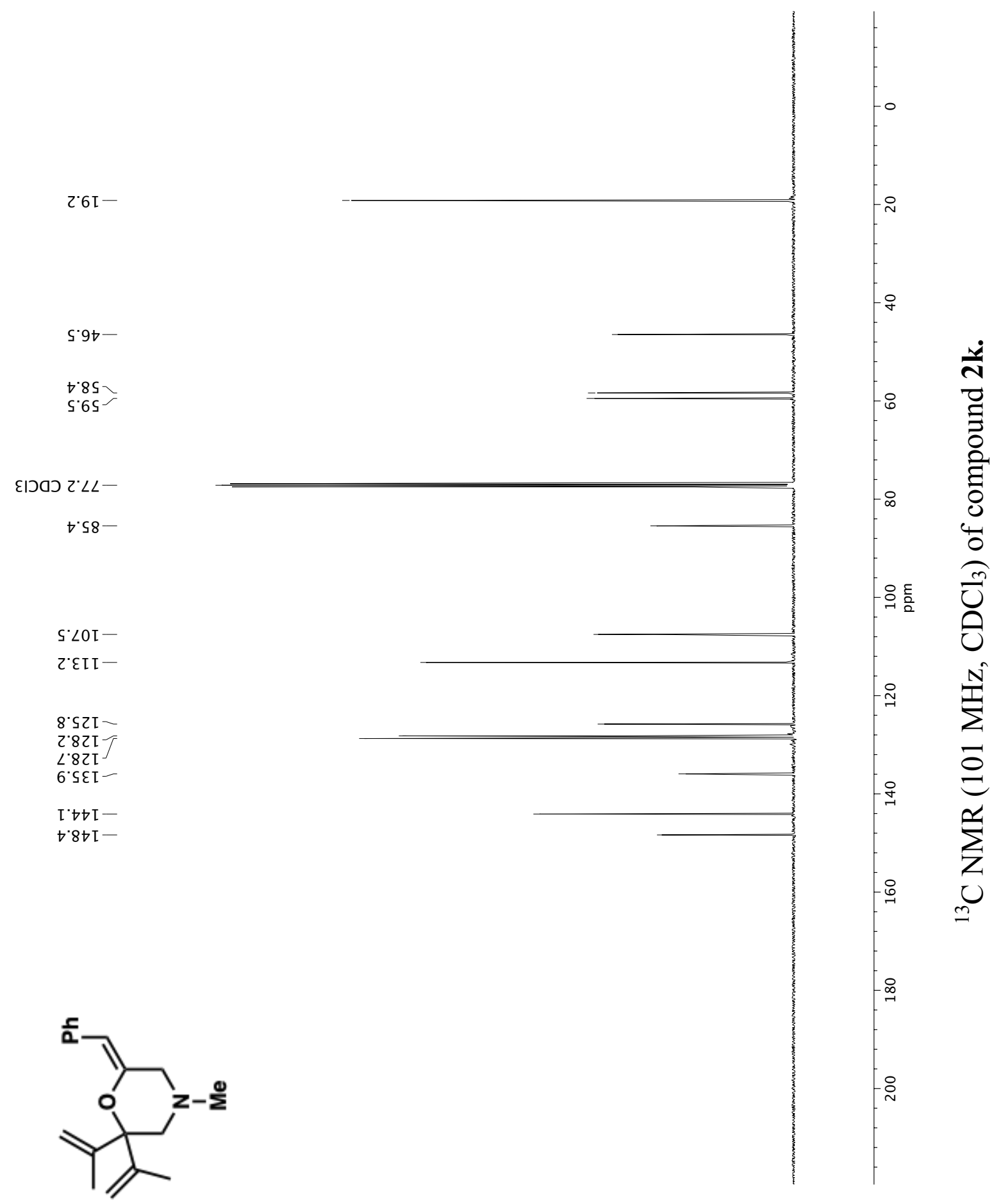


U४ $\angle$ L

ह9I I' 2

ह9I I'Z
9L乙I' 2

$\varepsilon 0 t I^{\prime} 2$

$\angle S Z \varepsilon^{\circ} Z$

$0 \angle \varepsilon \angle ' Z$

999L乙

0 L08 2

乙ع08 $ح$

૦દદ8 $8^{\circ} 乙$

I ऽ๕8 $8^{\circ} \mathrm{Z}$

乙L\&8 $ح$

$\angle \nabla \nabla \tau^{\prime} \varepsilon$

s $9 \nabla \tau^{\prime} \varepsilon$

$\angle 9 \angle Z^{\circ} \varepsilon$

५ $8 \angle Z^{\circ} \varepsilon$

8टโ9'

$\angle \supset\left[9^{\circ} \varepsilon\right.$

99โ9.

98 [9 $\varepsilon$

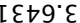

$\varepsilon 9 \pitchfork 9^{\circ} \varepsilon$

$8 \angle 88^{\circ} \varepsilon$

$\neg 006^{\circ} \varepsilon$

$\angle 2$ L6 $^{\circ} \varepsilon$

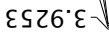

2S6I' $\bullet$

96几で

0七七で

2885' $t$

ع065 $\rightarrow$

6265. $t$

$056 S^{\circ} \nabla$

$6 \triangleright 06^{\circ} \bullet$

$8 \angle 06^{\circ} \nabla$

$8606^{\circ} t$

$\angle 2\left[6^{\circ}{ }^{\circ}\right.$

$9992^{\circ} L$

$80 \angle Z^{\circ} L$

$6 \rightarrow \angle Z^{\circ} \angle$

$0 \varepsilon 8 \mathrm{C}^{\circ} \mathrm{L}$

$9 S 82^{\circ} L$

$2882^{\circ} \angle$

6ऽદ ' $\angle$

乙6દ $\mathcal{L}^{\circ}-$

$\angle 8 \nabla \varepsilon^{\circ} \angle$

9ISE' $L$

ऽ乙9ع $\angle$

โ99\& $L$

$\angle \angle 9 \varepsilon^{\circ} \angle$

$\nabla 980^{\circ} \mathrm{L}$

$088 t^{\circ} \mathrm{L}$

$968 t^{\circ} \mathrm{L}$

$\angle I 6 t^{\circ} \angle$

9205. $\angle$

6SOS $L$

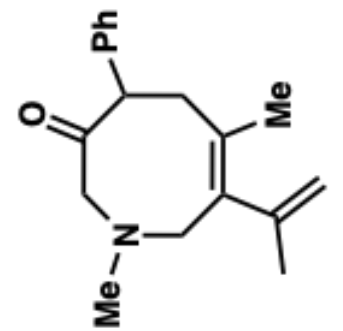

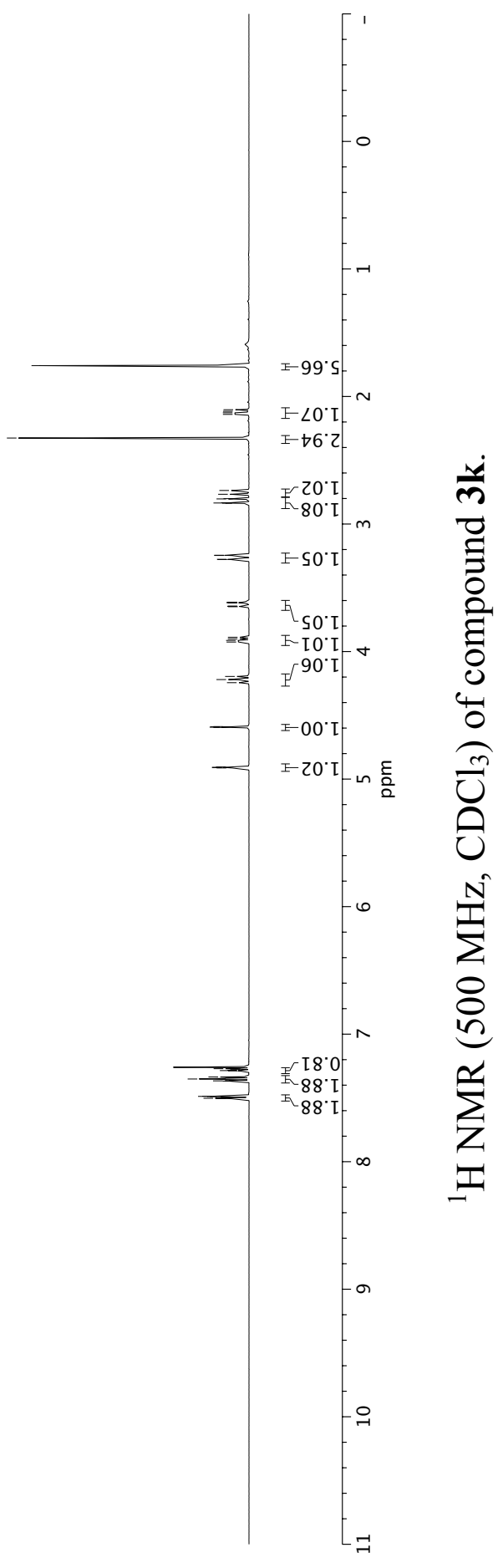




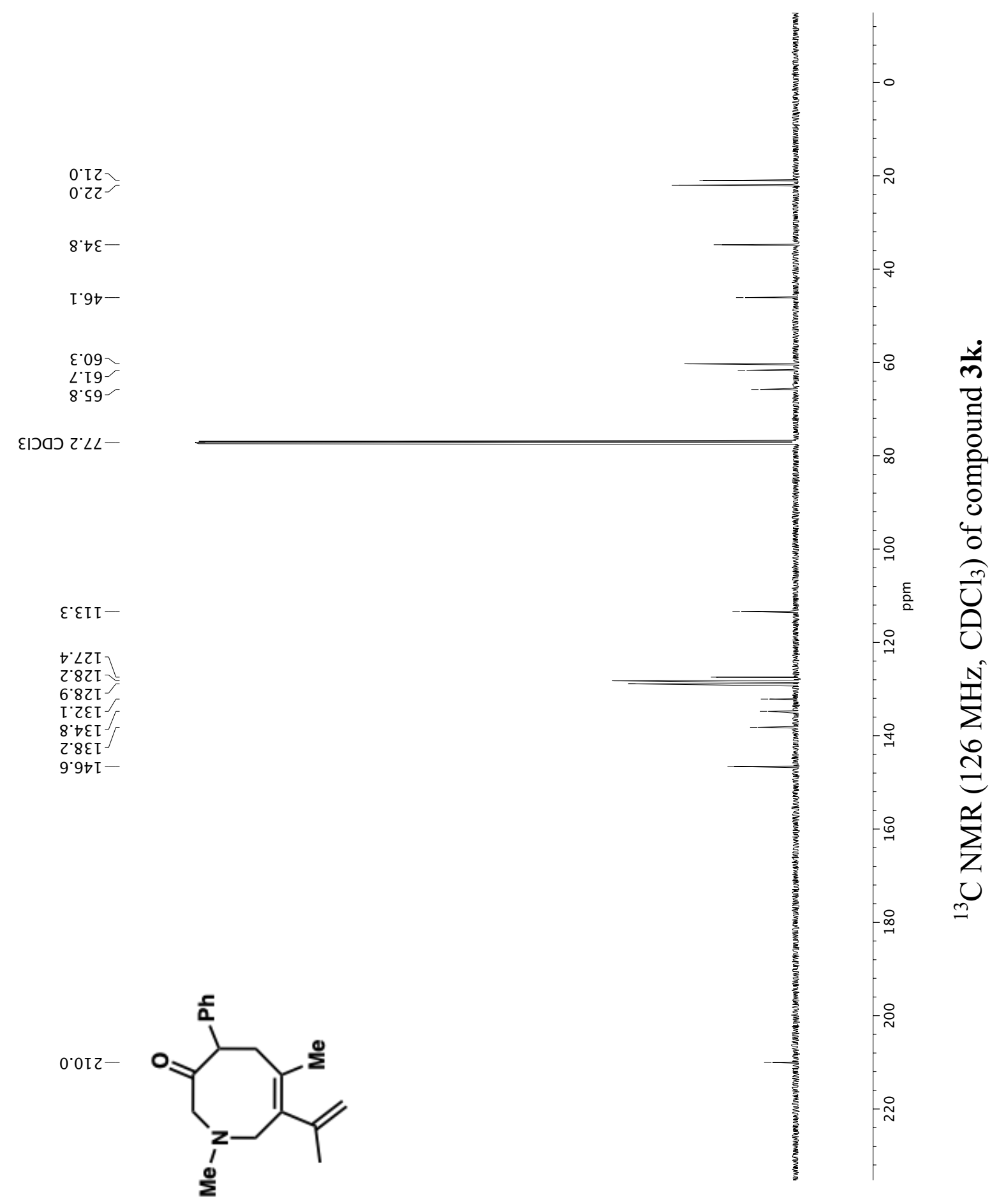



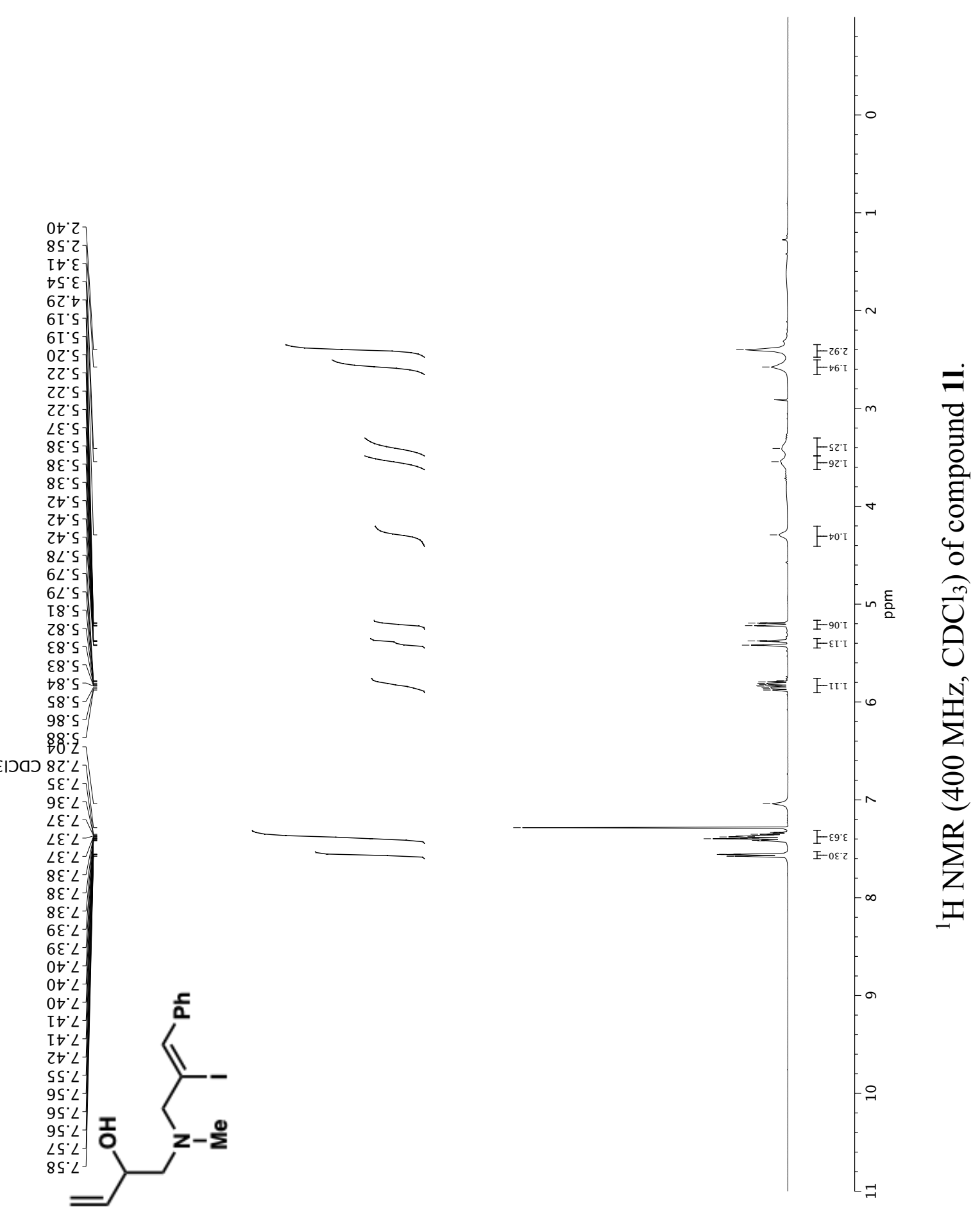

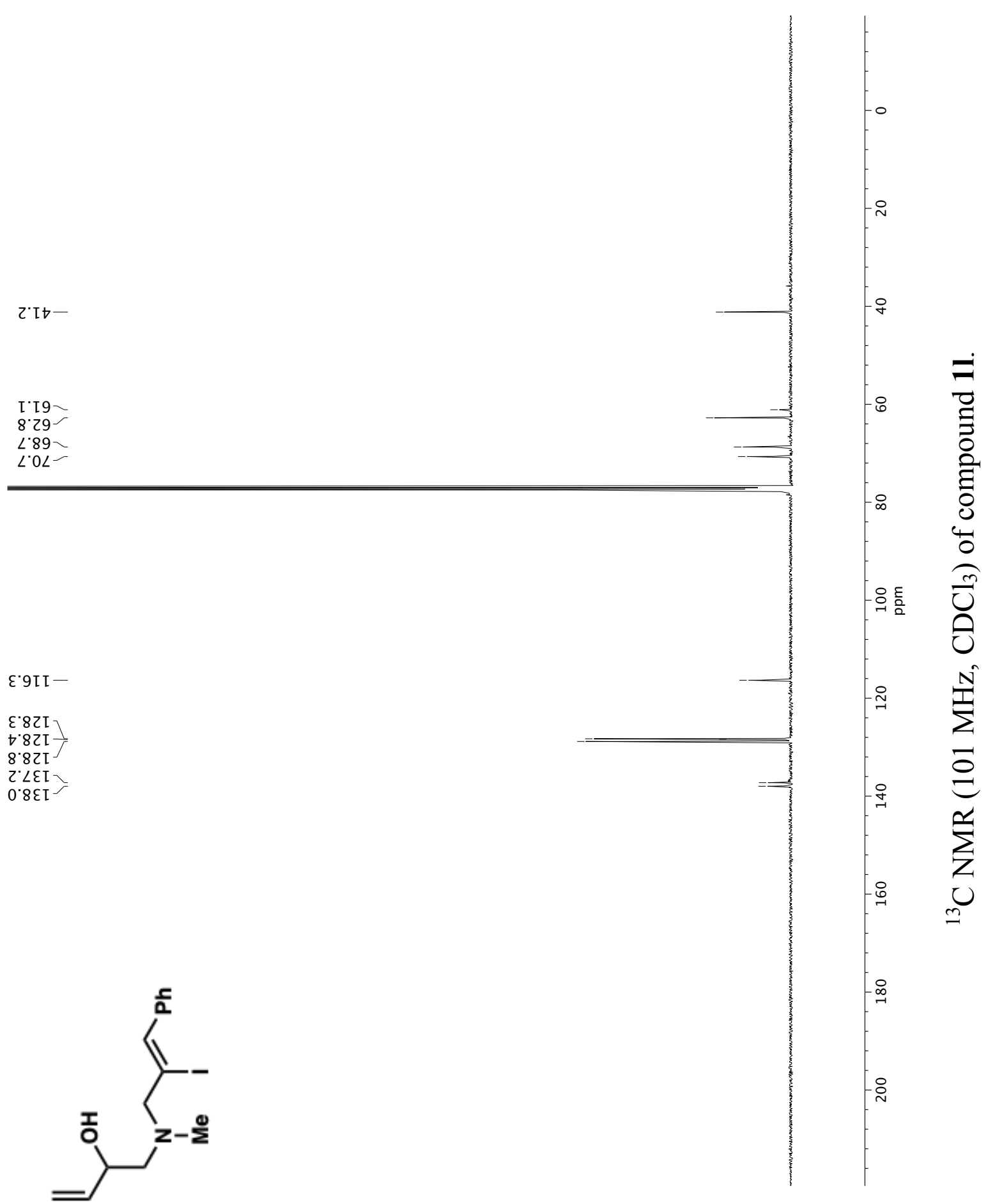

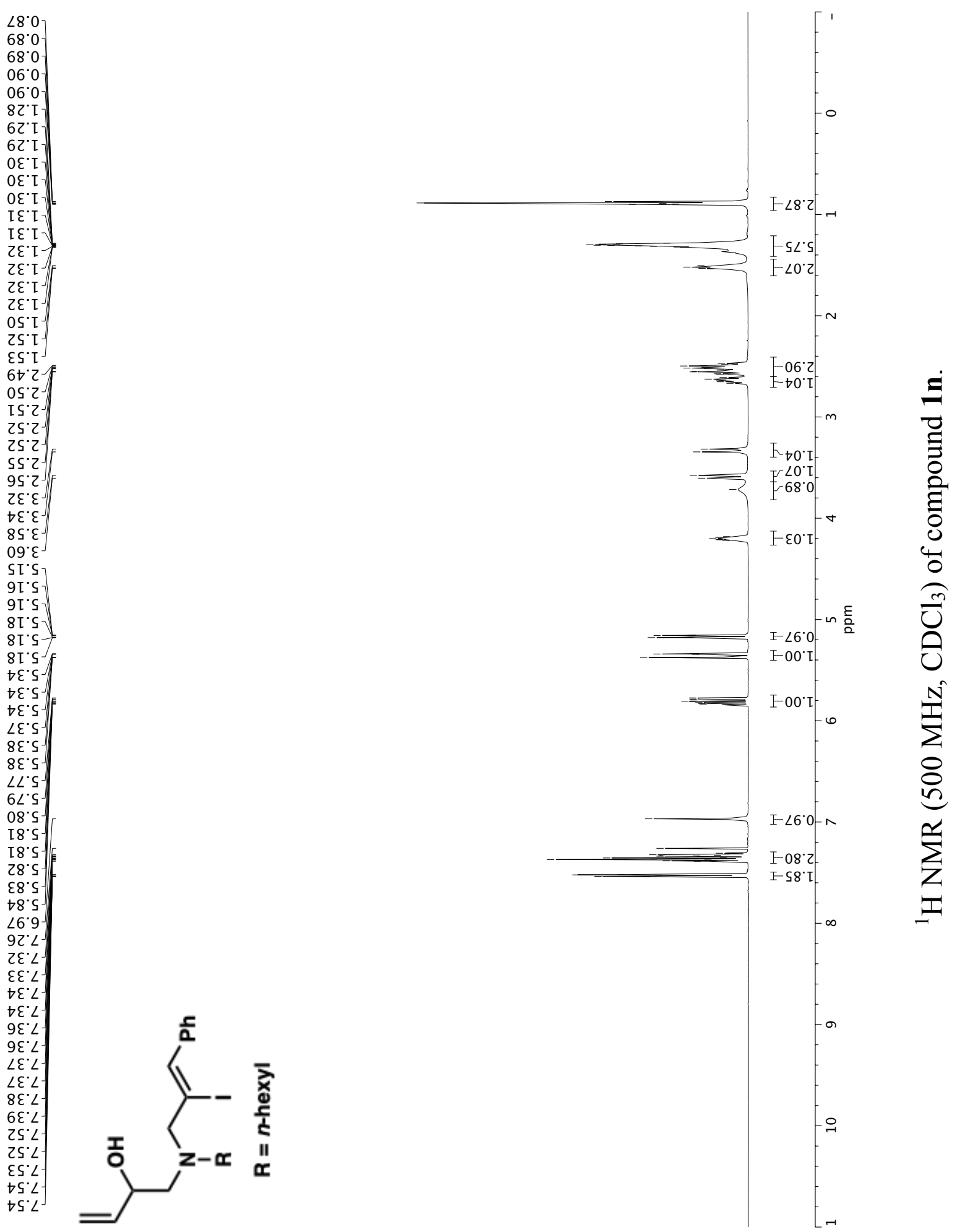
$\tau \cdot t I-$

8.22

9.92

$8^{\circ} \mathrm{IE}^{-}$

t`६ऽ-

L.6S-

9.29
8.89

9. $\angle 0 I-$

I.9 I I -

$\varepsilon .8 Z \mathrm{I}$

$\varepsilon 82 \mathrm{~T}$

$8.82 \mathrm{~L}$

$\varepsilon \cdot 9 \varepsilon \mathrm{I}$

S. $\angle E I J$

$28 \varepsilon \mathrm{I}$
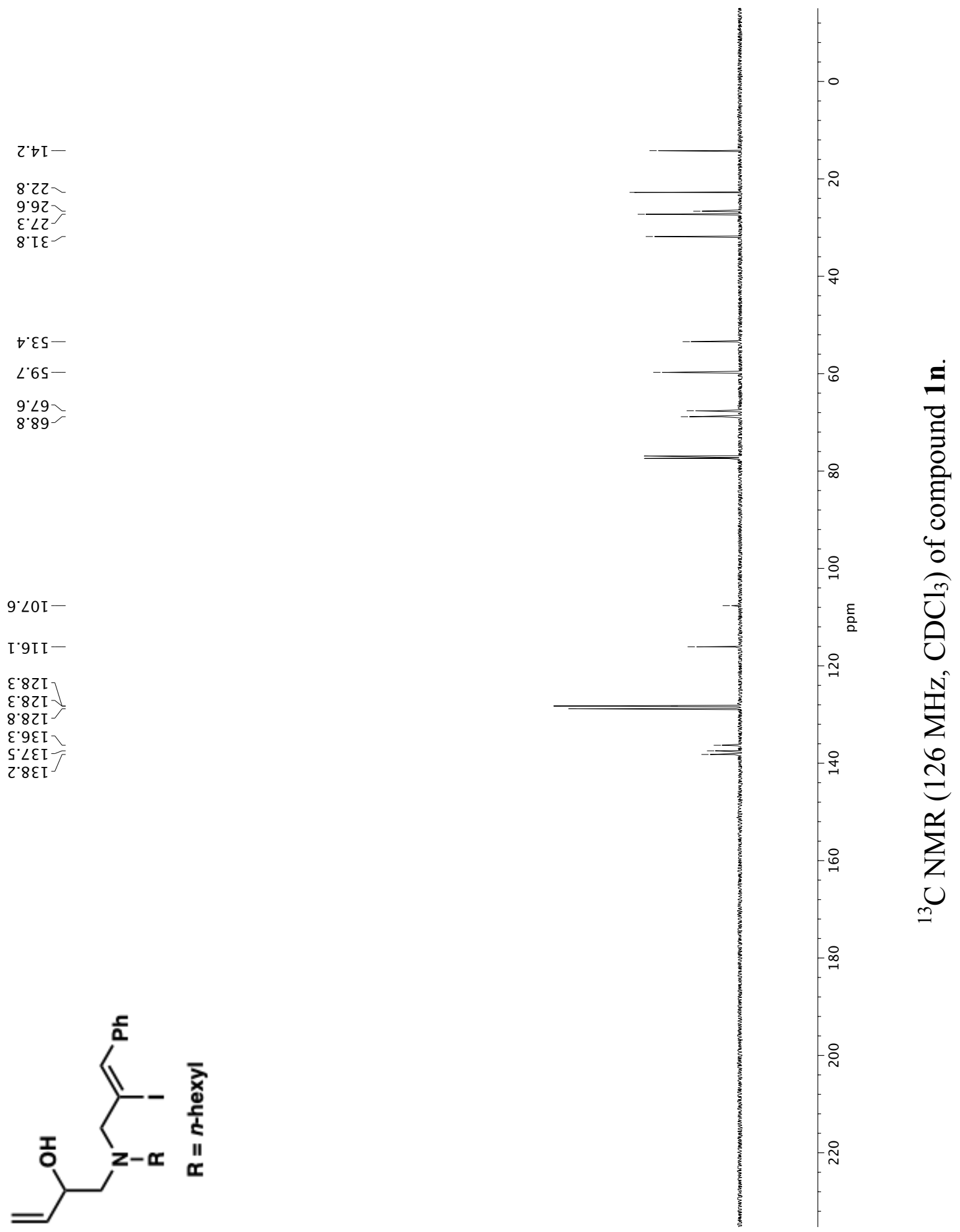

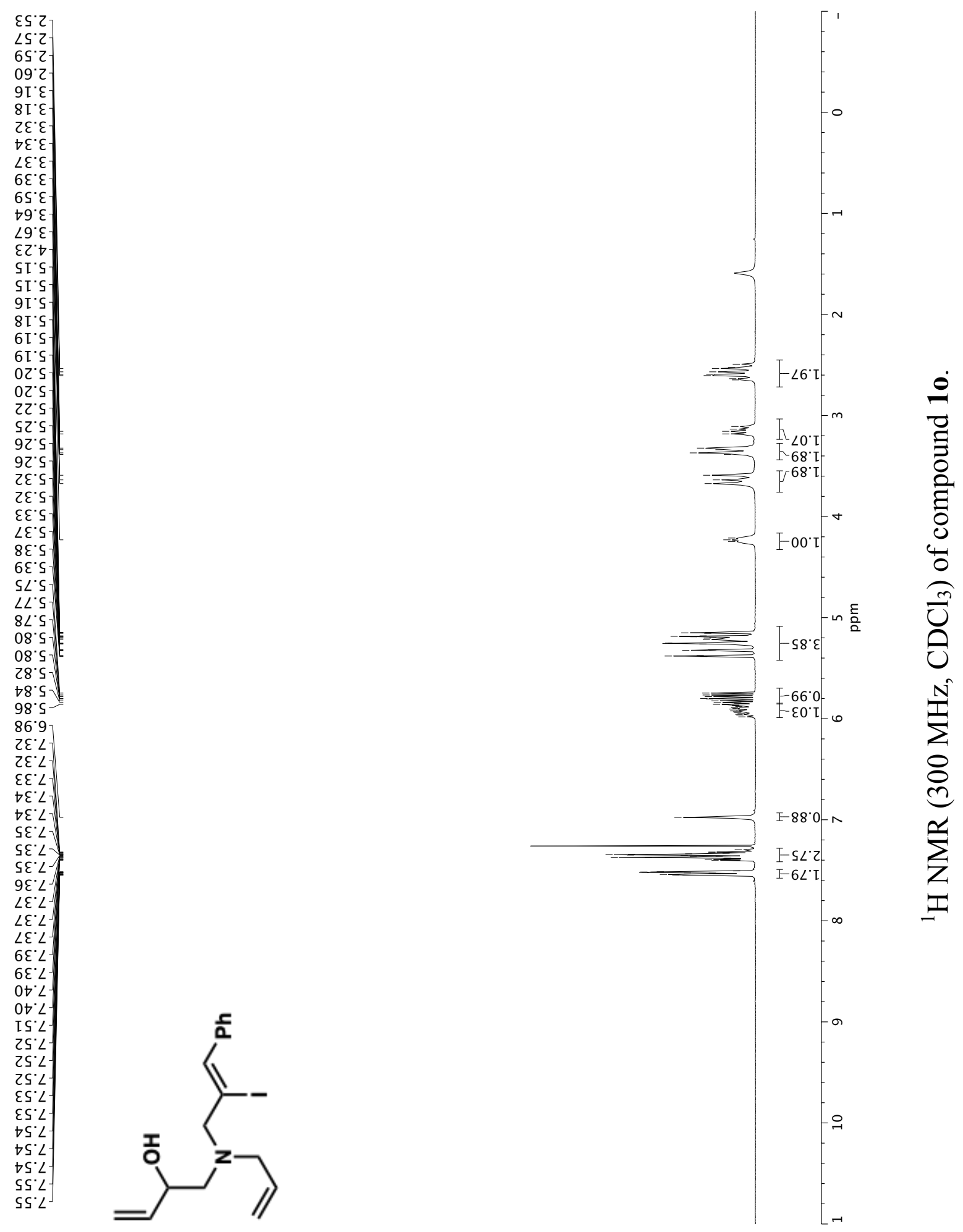


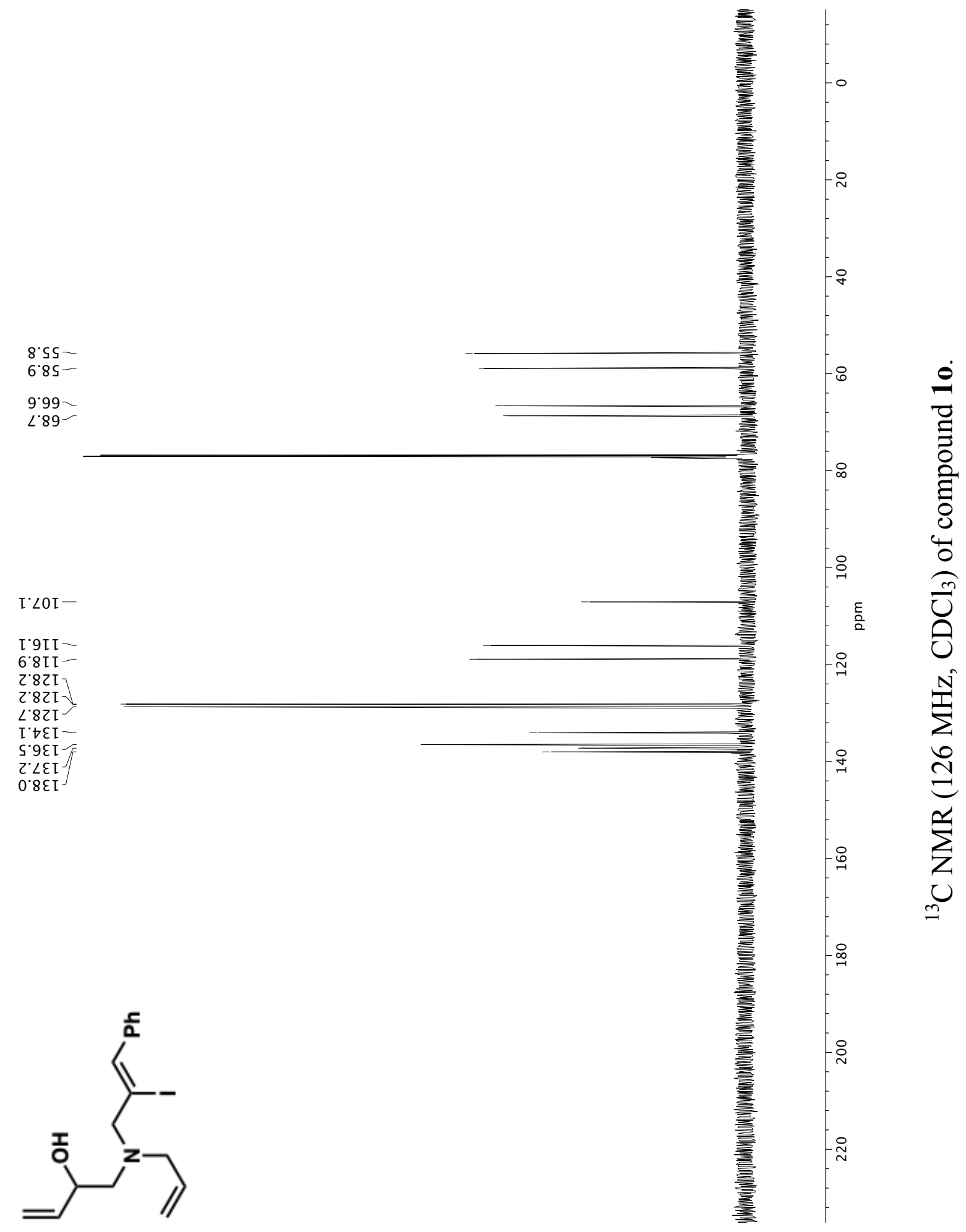




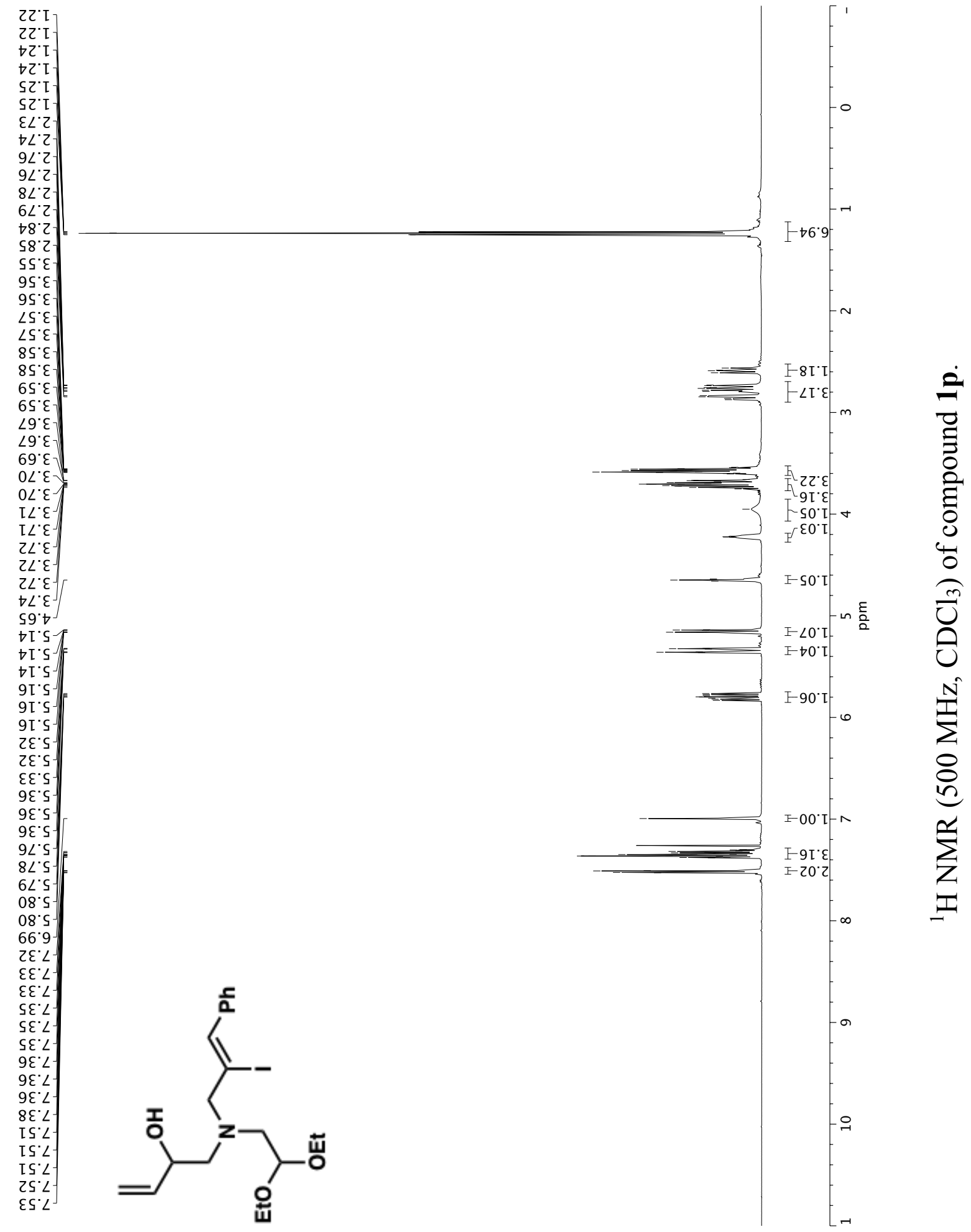


8. TOL -

$0 . \angle 0 \mathrm{I}-$

6.SII-

[. $82 \mathrm{I}$

$2.82 \mathrm{I}$
$2.82 \mathrm{I}$

$2.9 \varepsilon$

$\varepsilon \angle \varepsilon I-$

$0.8 \varepsilon \mathrm{I}$
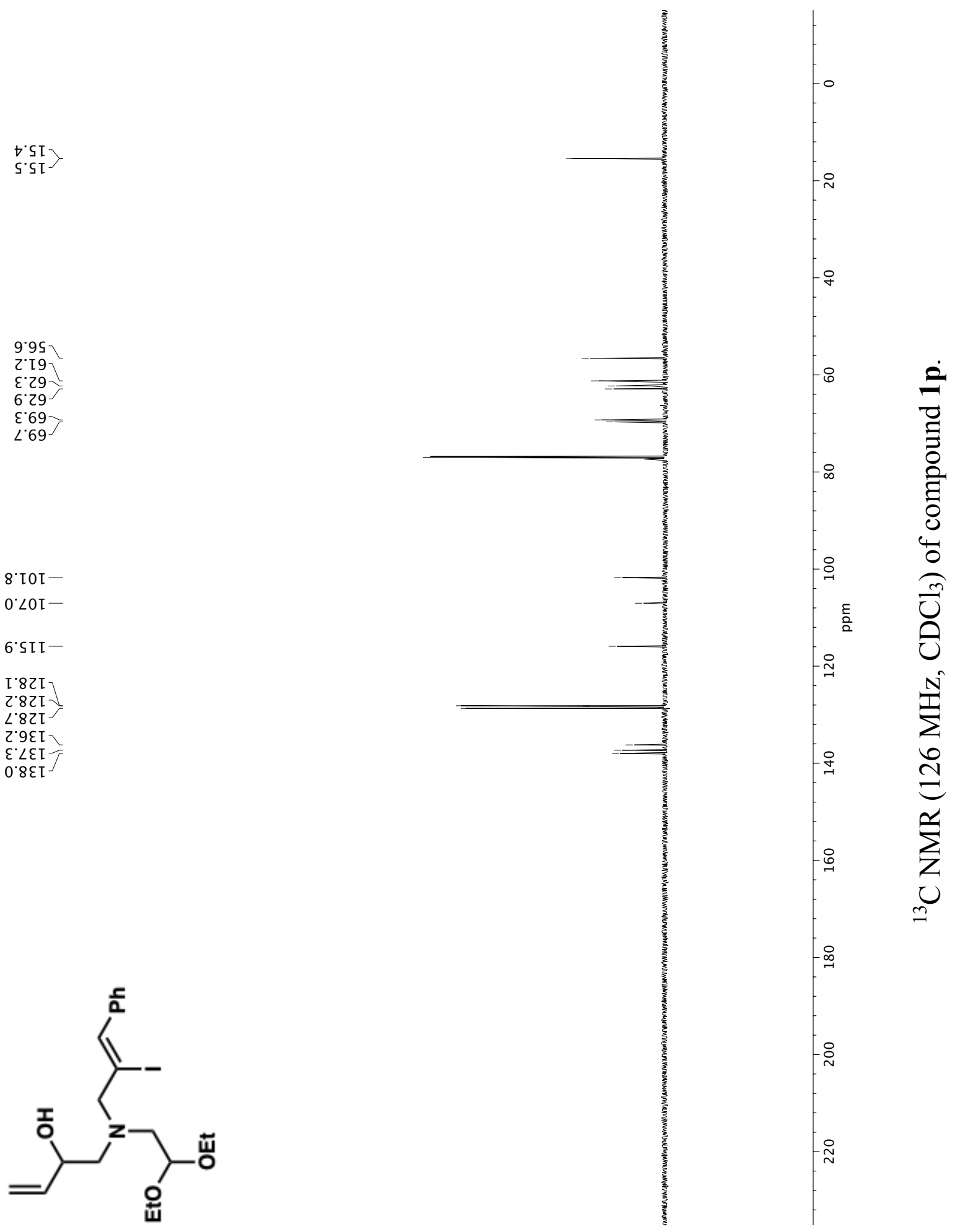

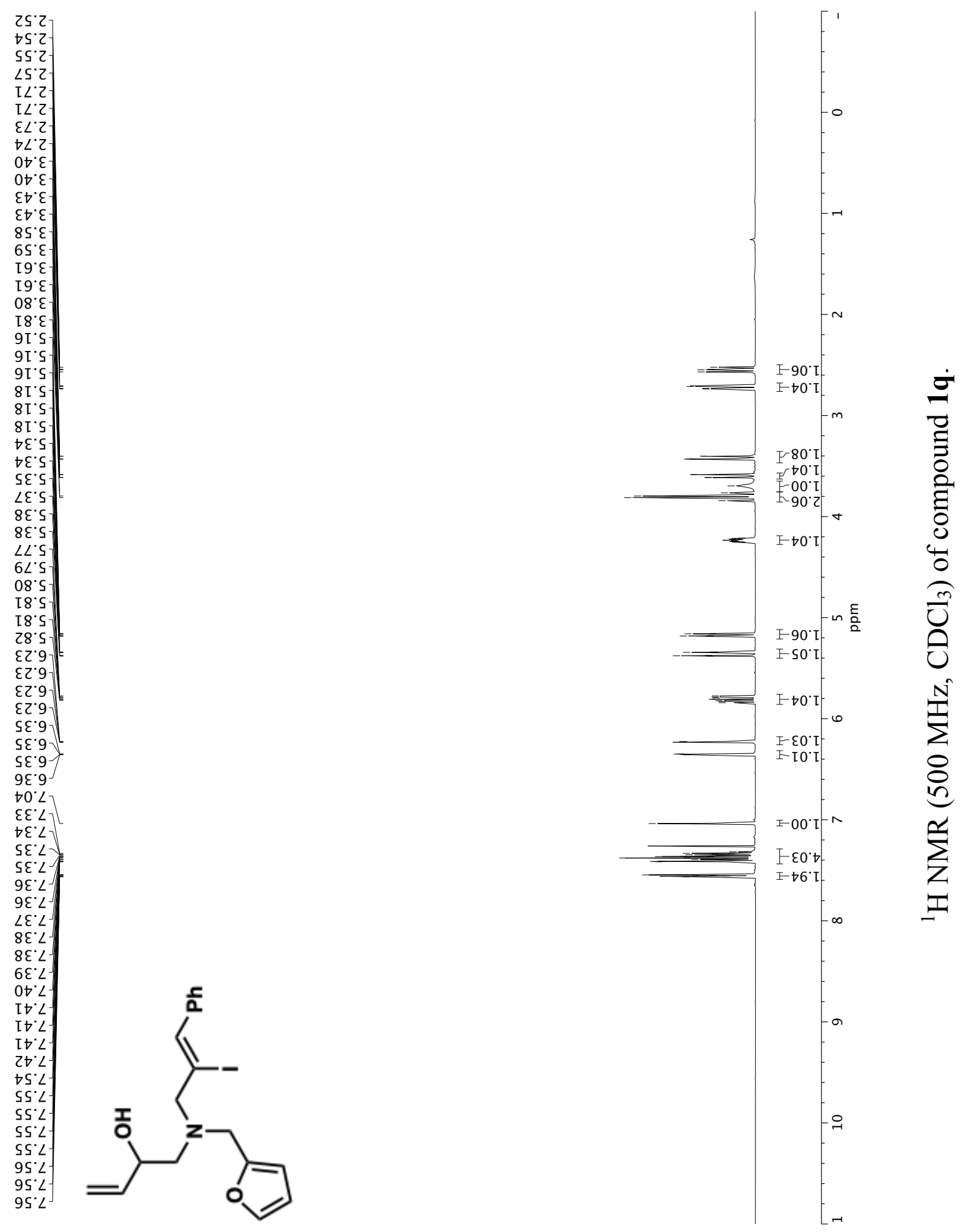


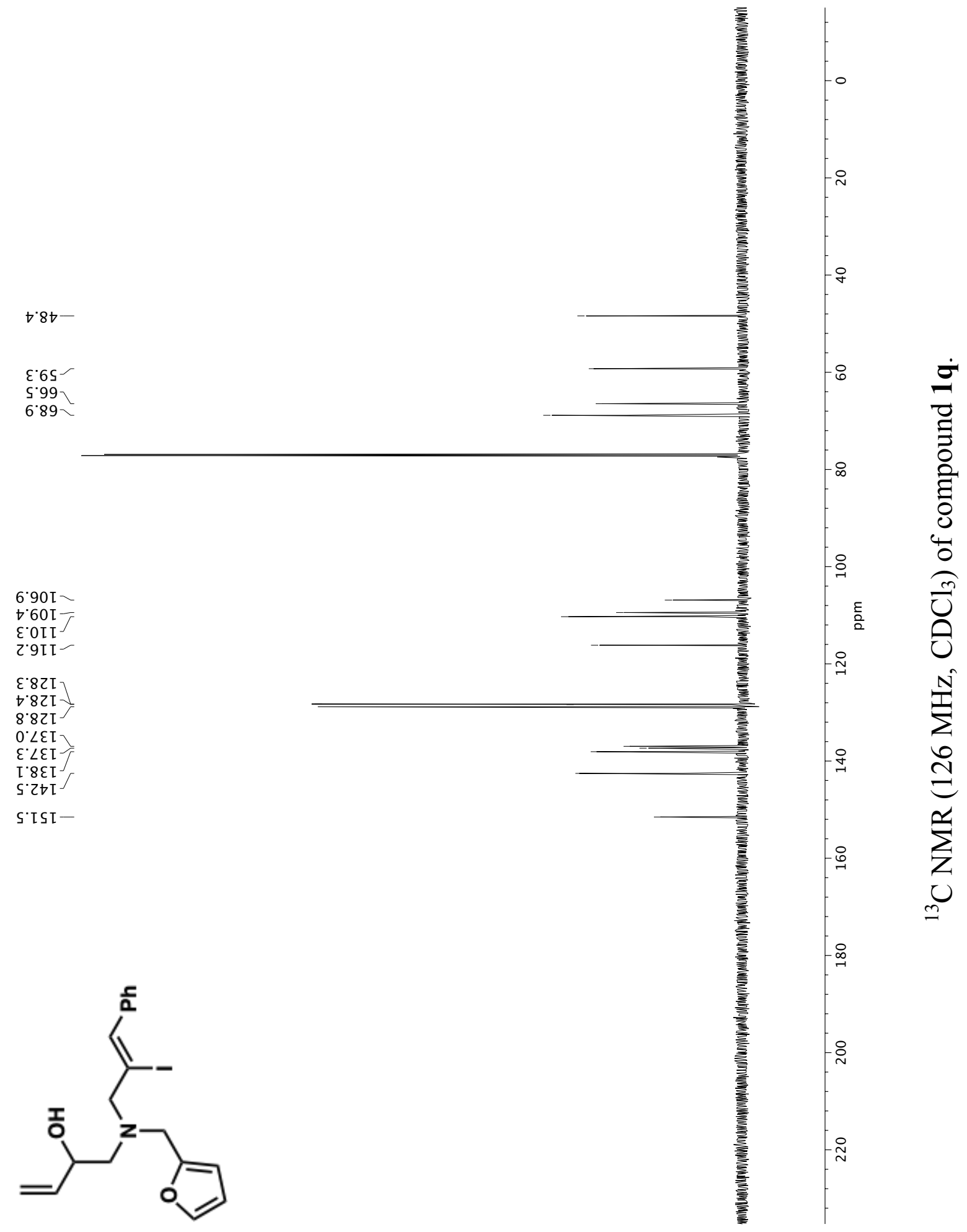



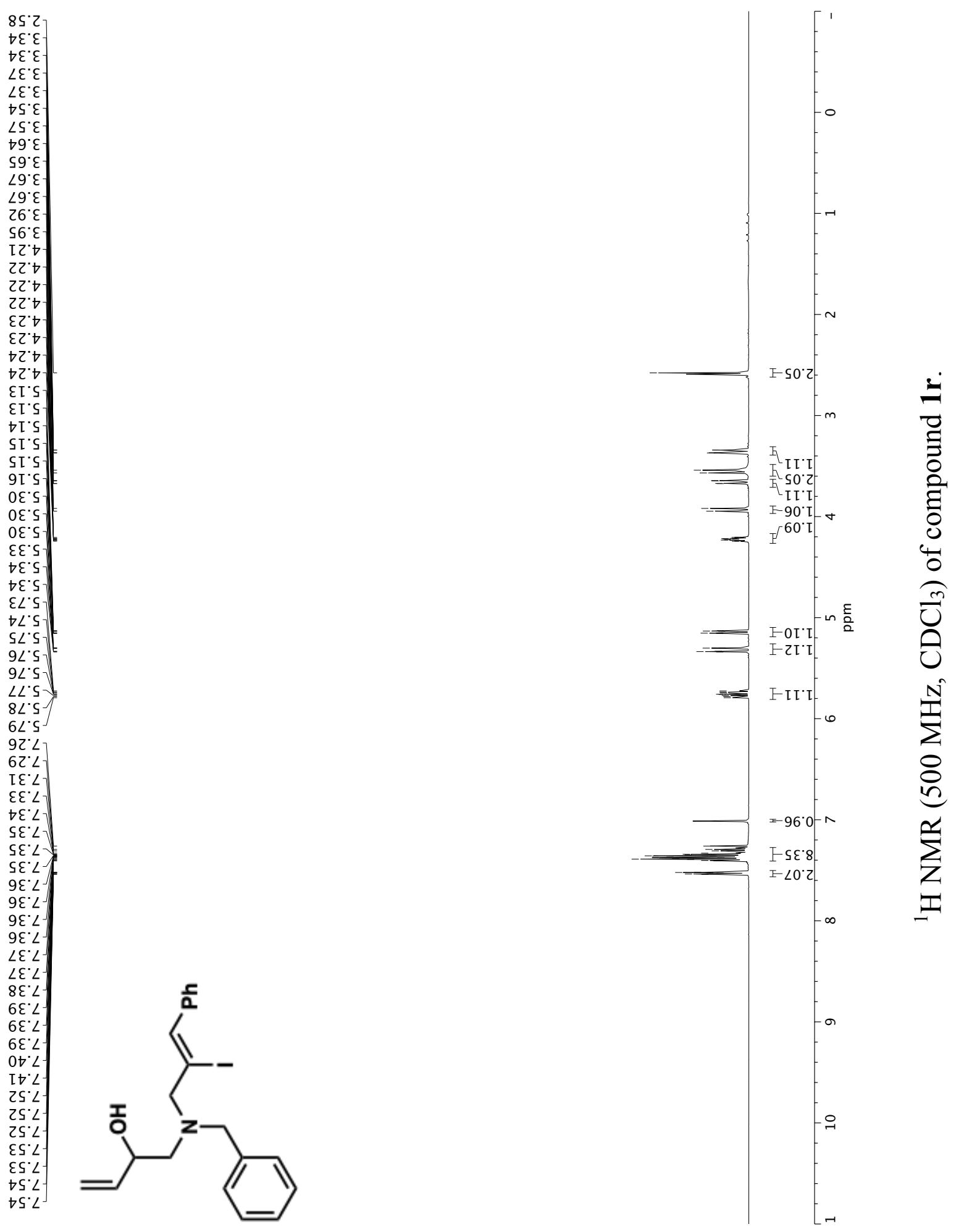


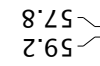

$0.29=$
$8.89-$

6.90I-

I. 9 I I -

$S^{\prime} \angle Z I_{7}$

$2.82 \mathrm{I}$

$\varepsilon 82 \mathrm{I}$

$582 \mathrm{~L}-$

$\angle 8 Z I-$

S.6ZI

$0 \angle \varepsilon[-$

$\varepsilon<\angle \varepsilon I$

$\angle \angle E I$

$0.8 \varepsilon \mathrm{I}$

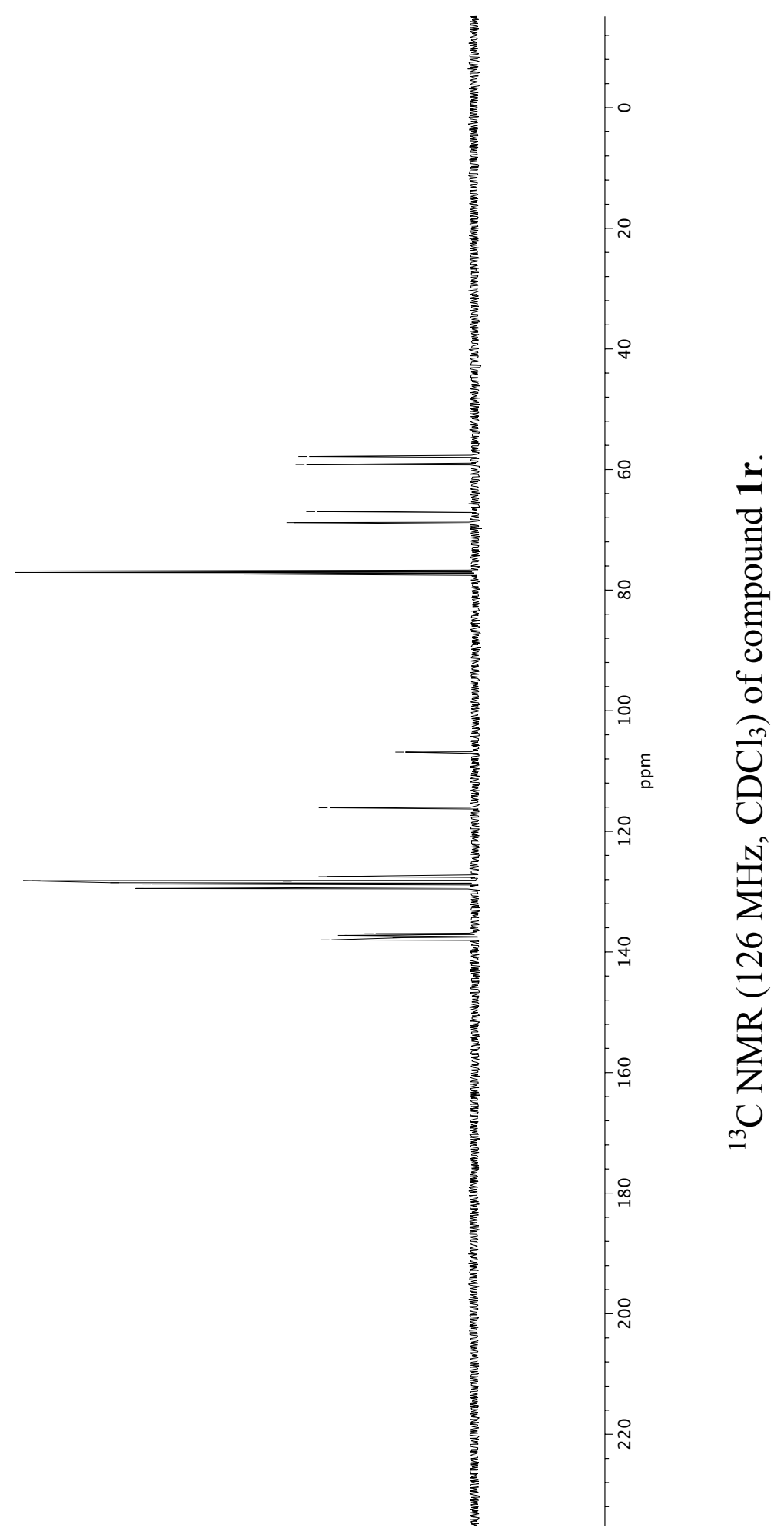



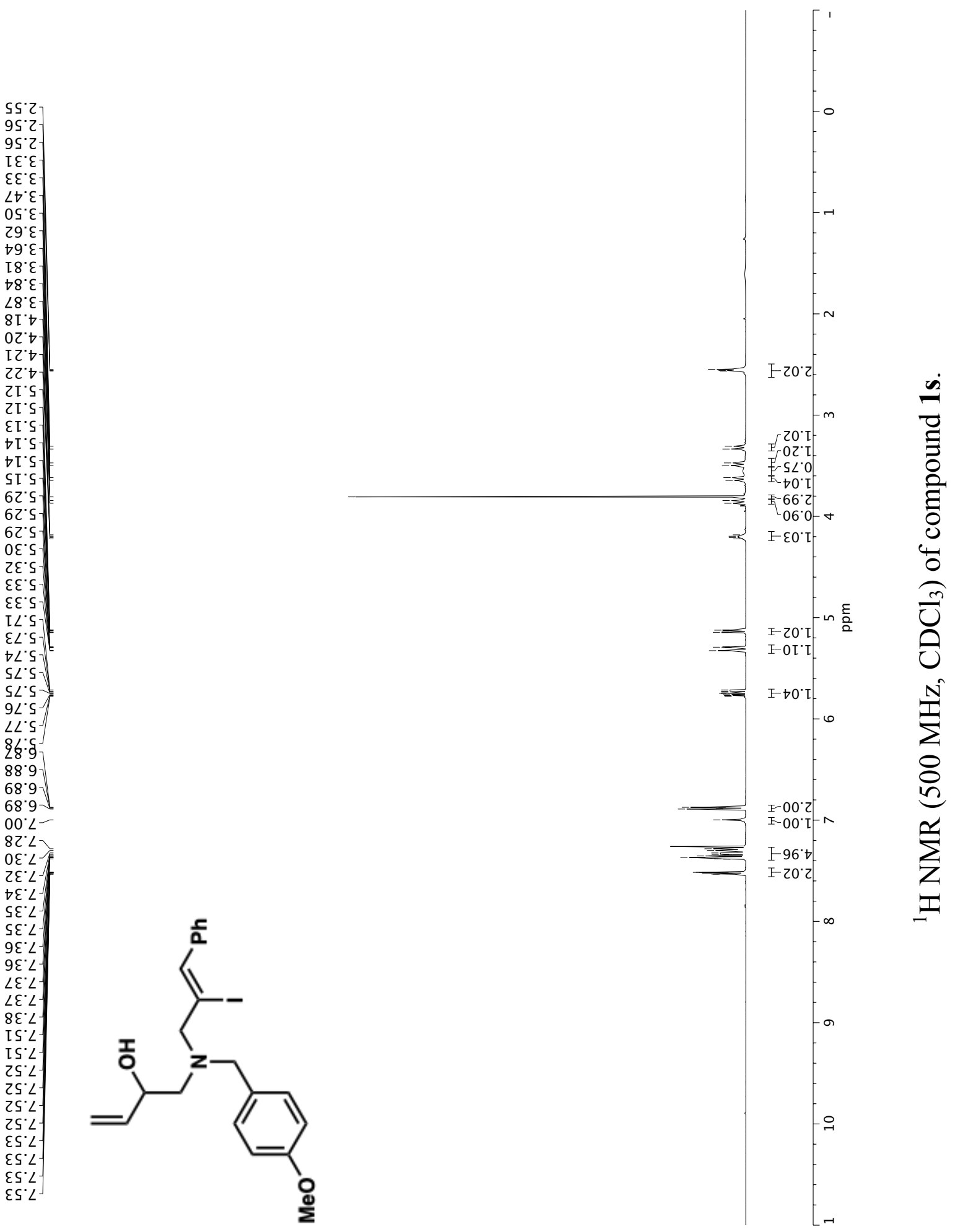


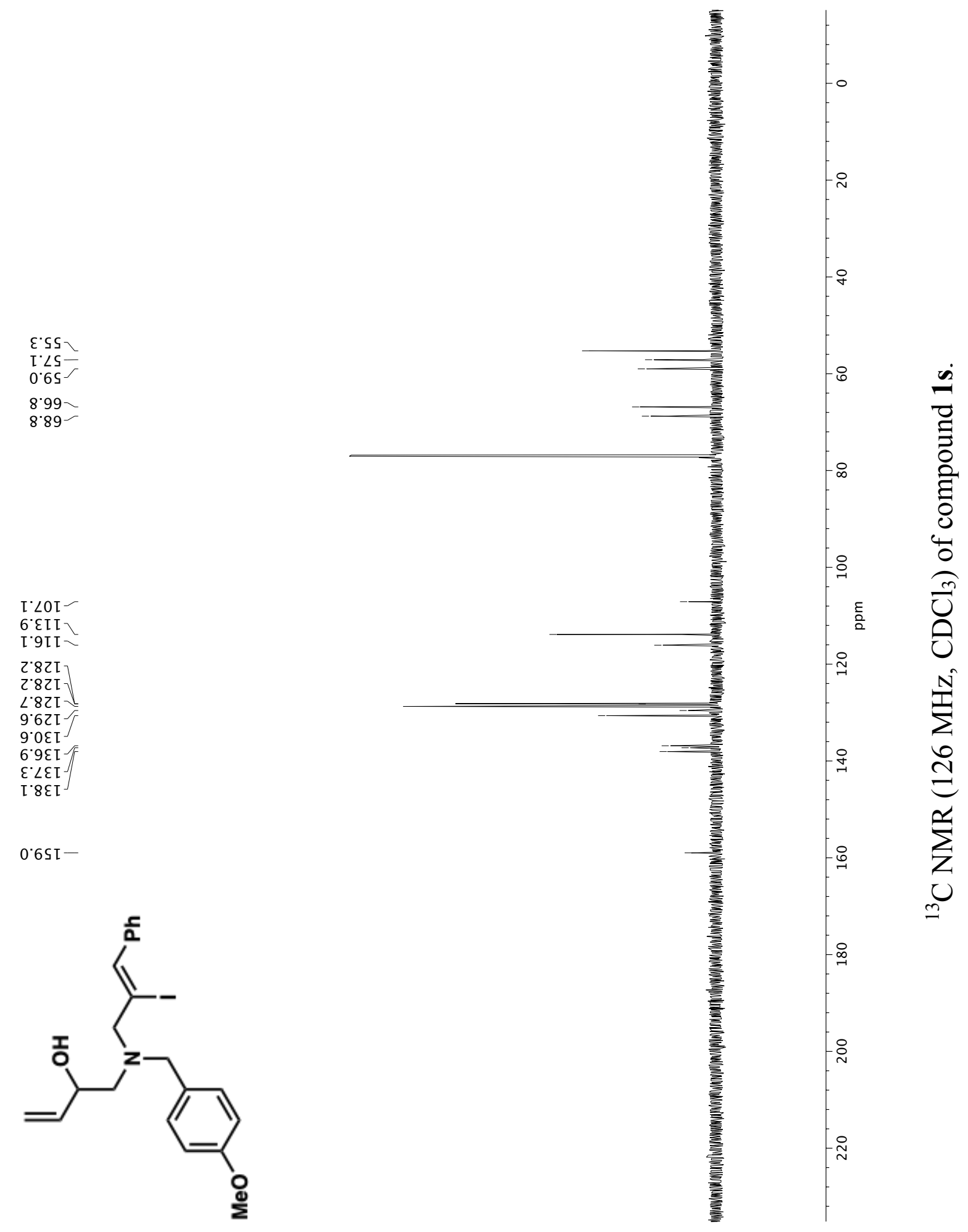



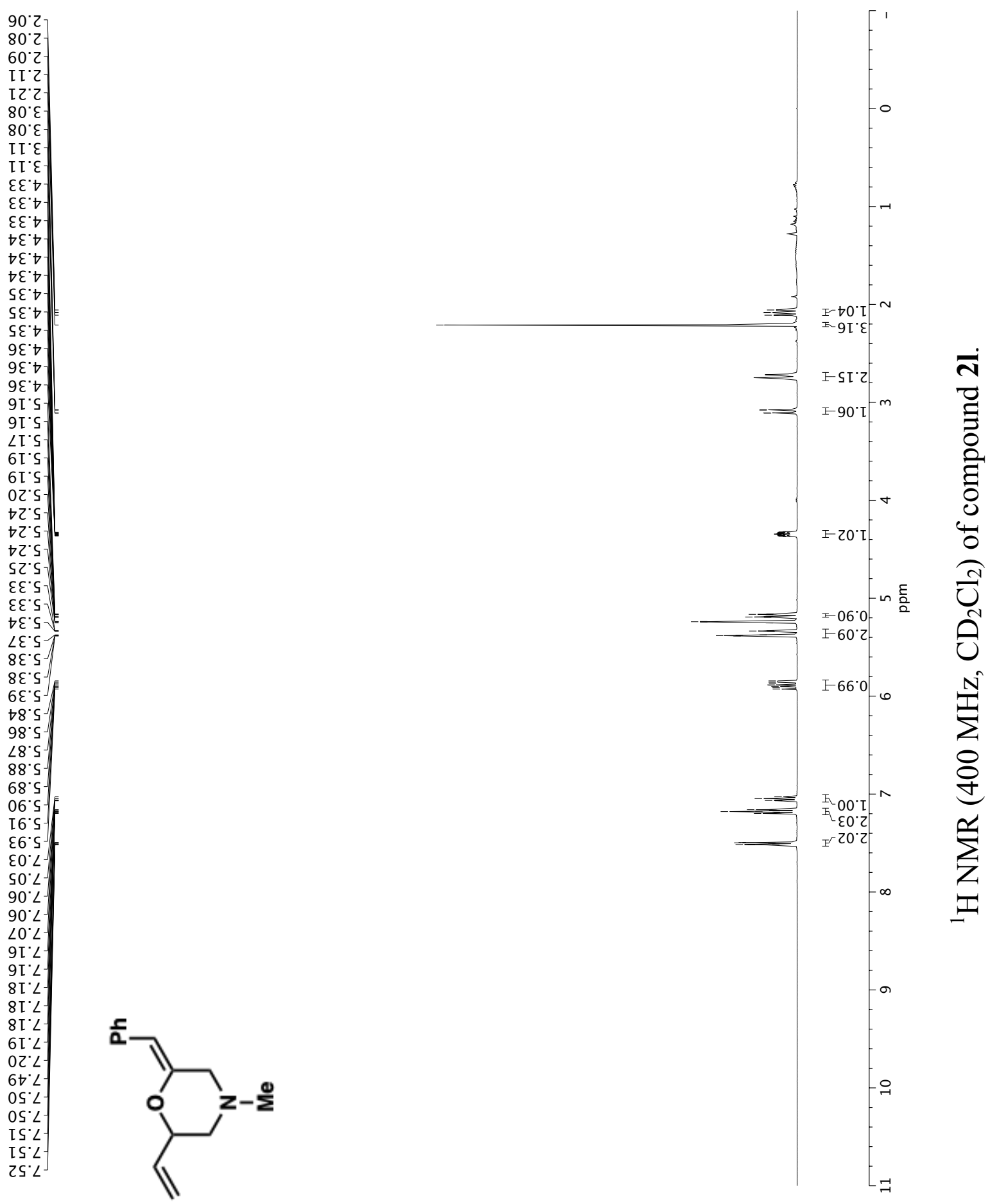


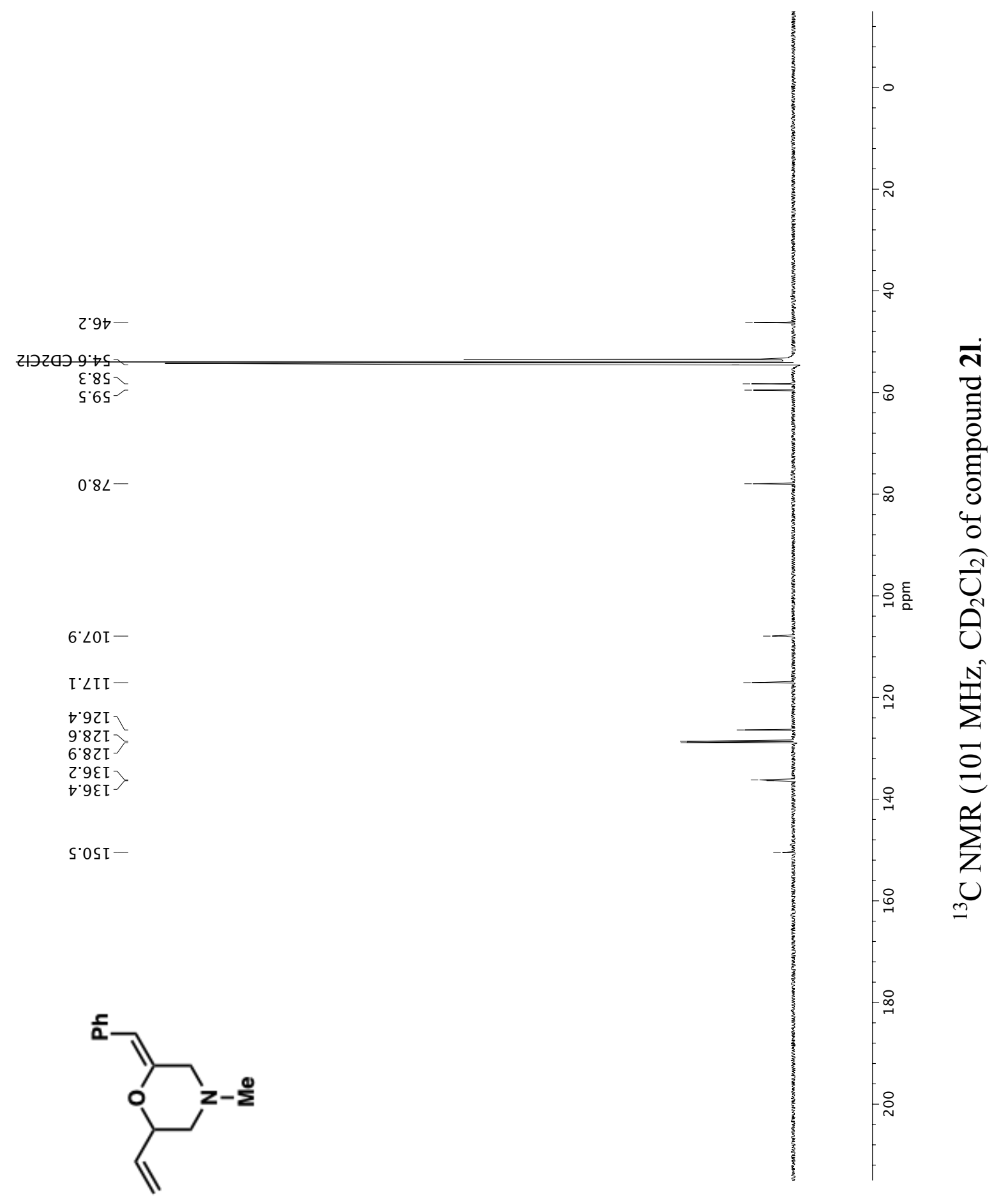



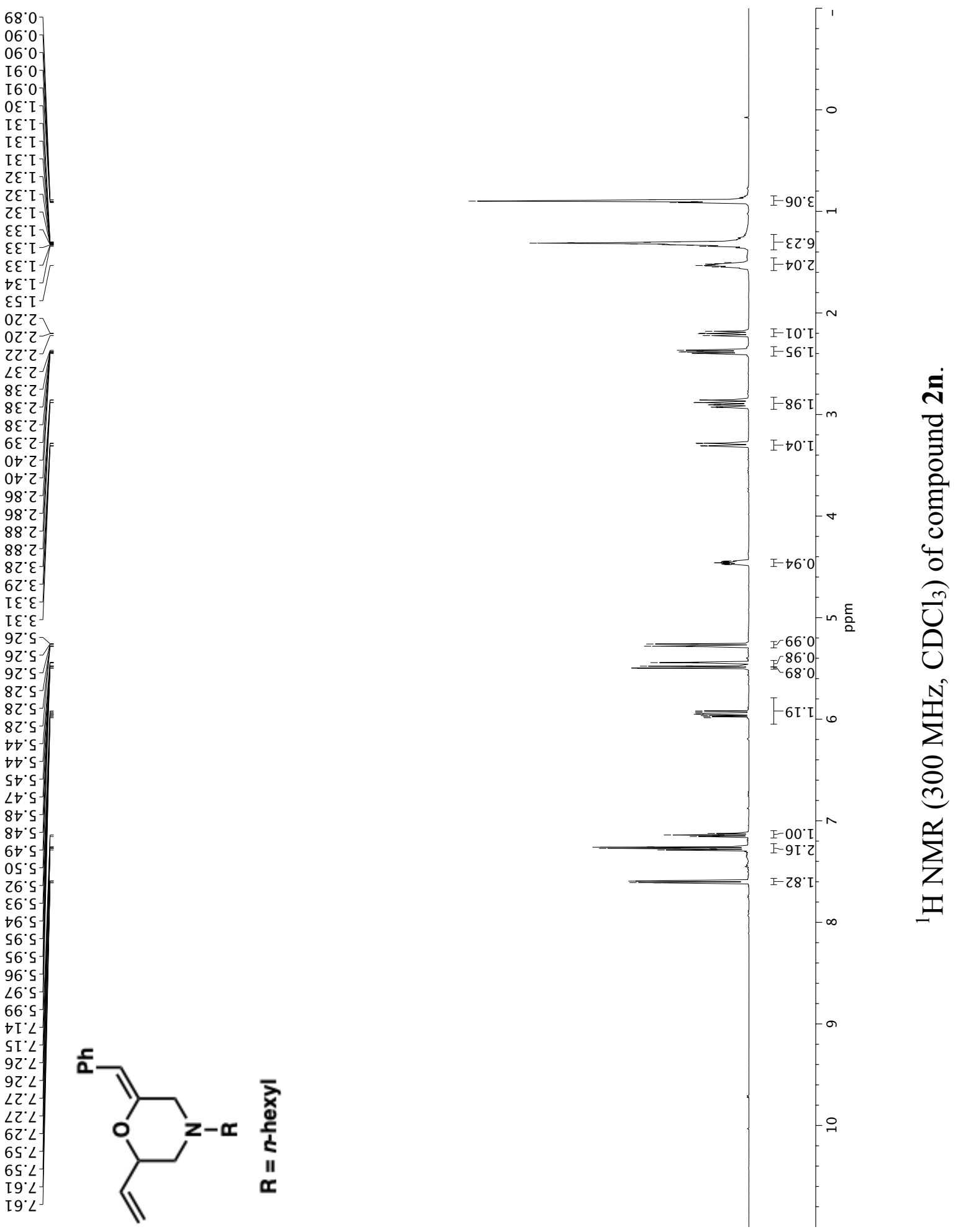
$I^{*} \triangleright I-$

9.22

9.92

8. $[\varepsilon-$

$\varepsilon \cdot 9 S$
$\varepsilon \cdot \angle S-$

985

$\mathrm{Z}^{\circ} 80$ I -

$0 \cdot \angle I I-$

6.SZI

I. $82 \mathrm{I}$
$\mathrm{c} .8 \mathrm{I}$ I

S.SEL

L'SEI

$s \cdot 6 t I-$

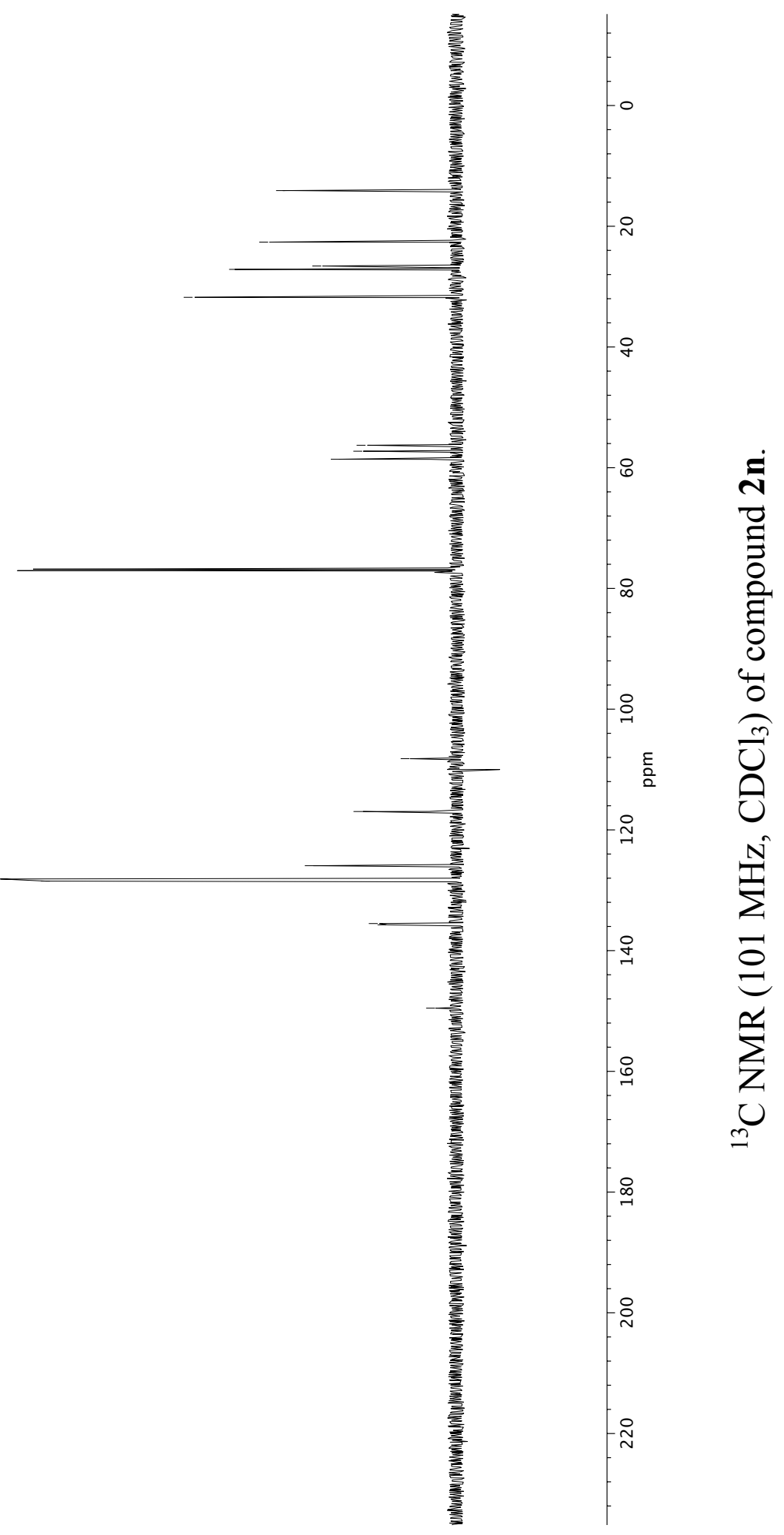

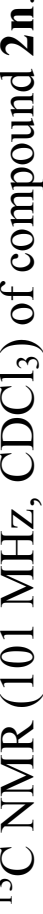



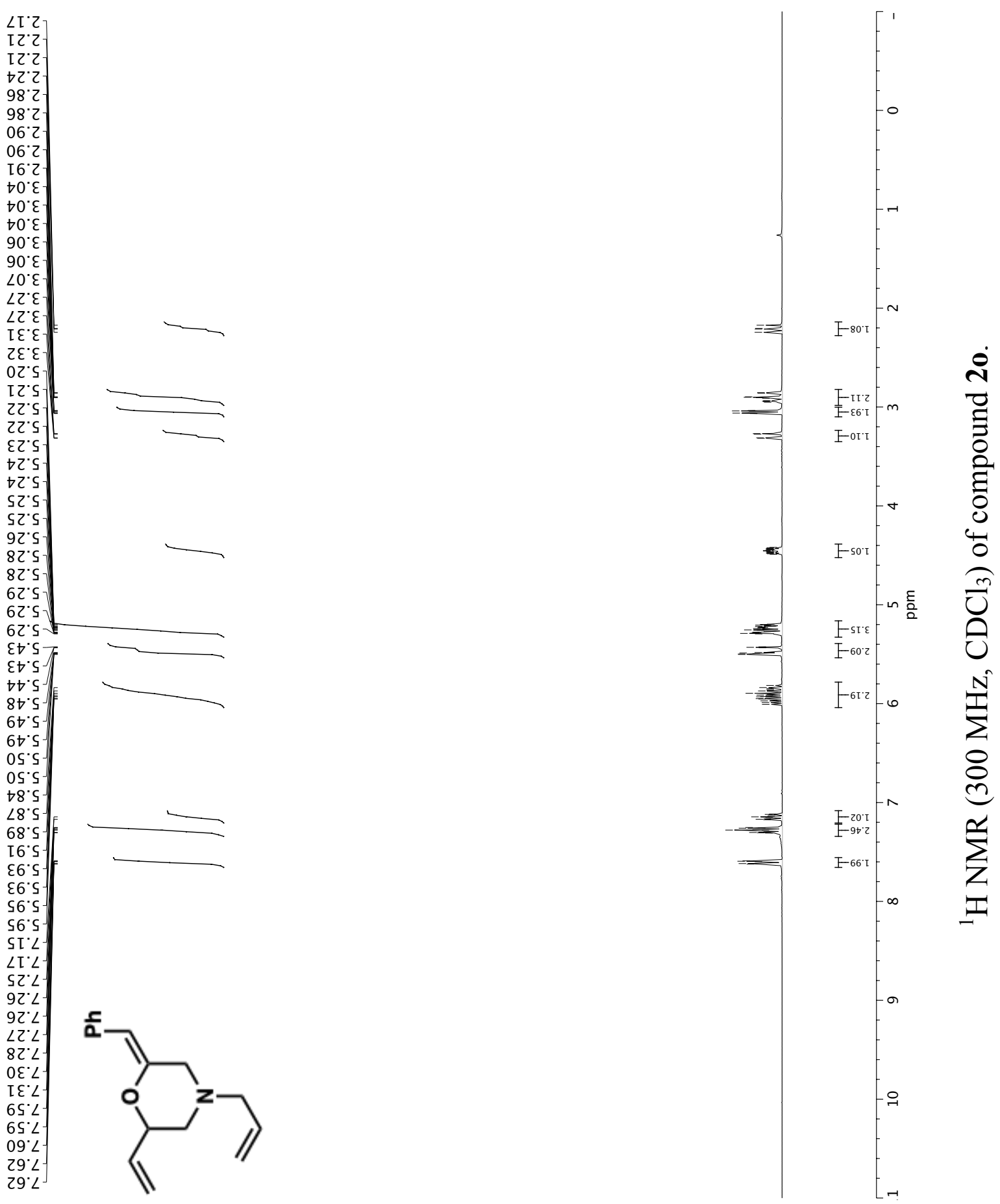
0.95

6.95

t. 19

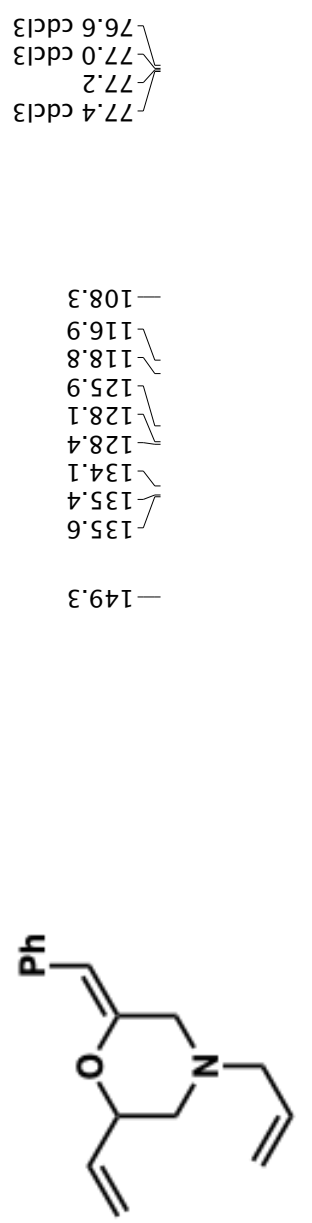

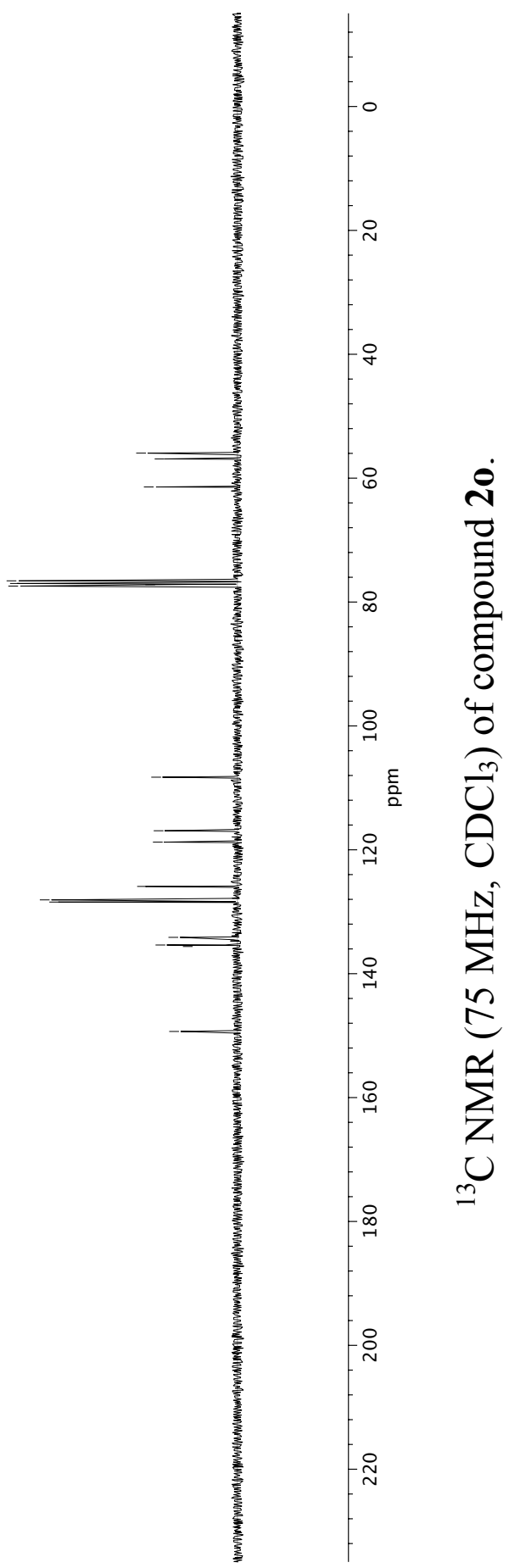



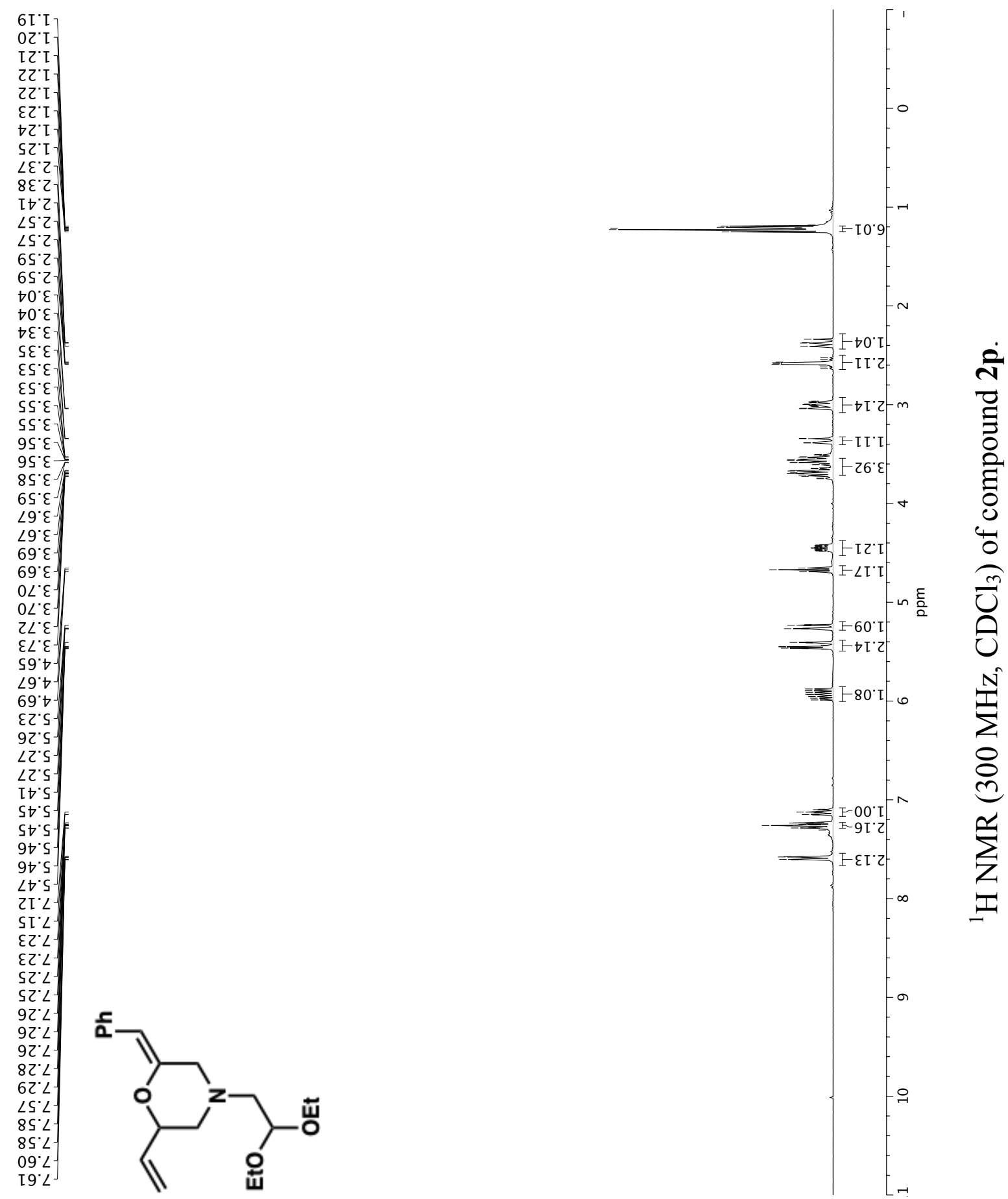


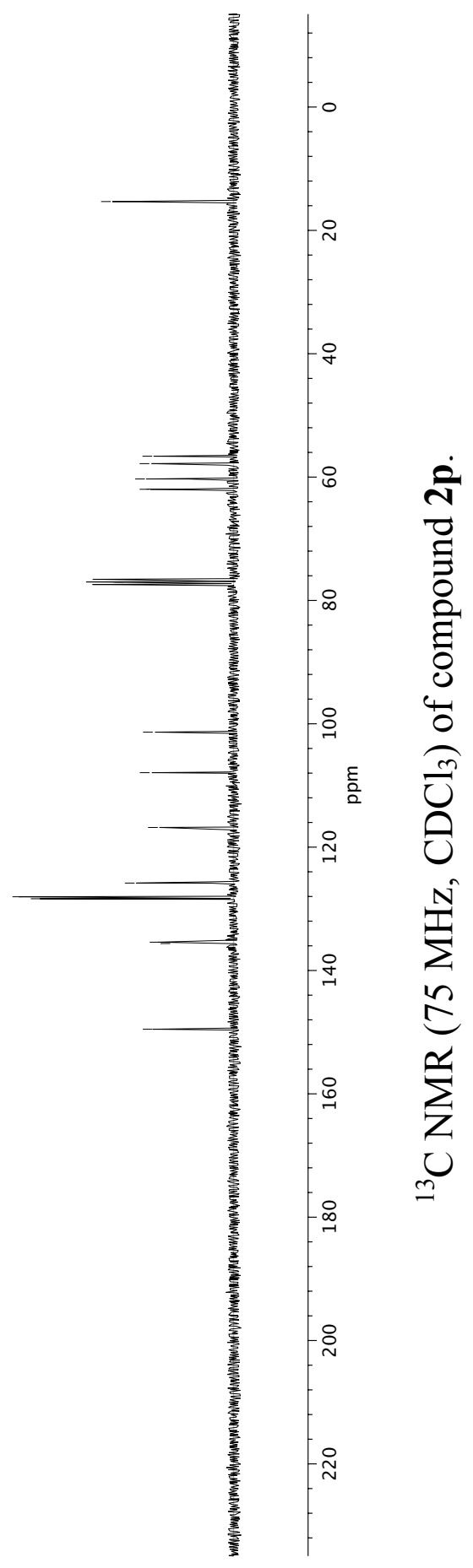



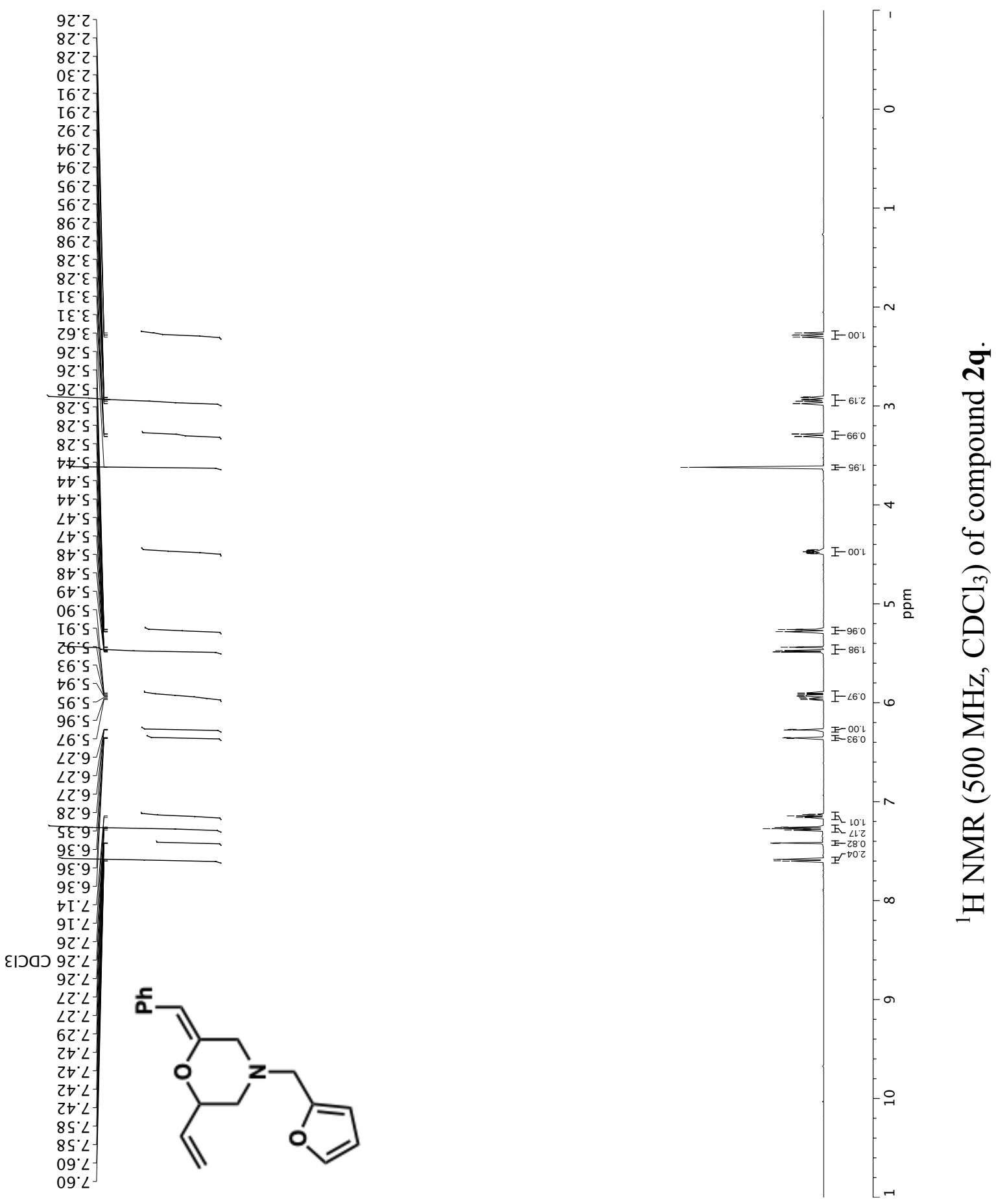

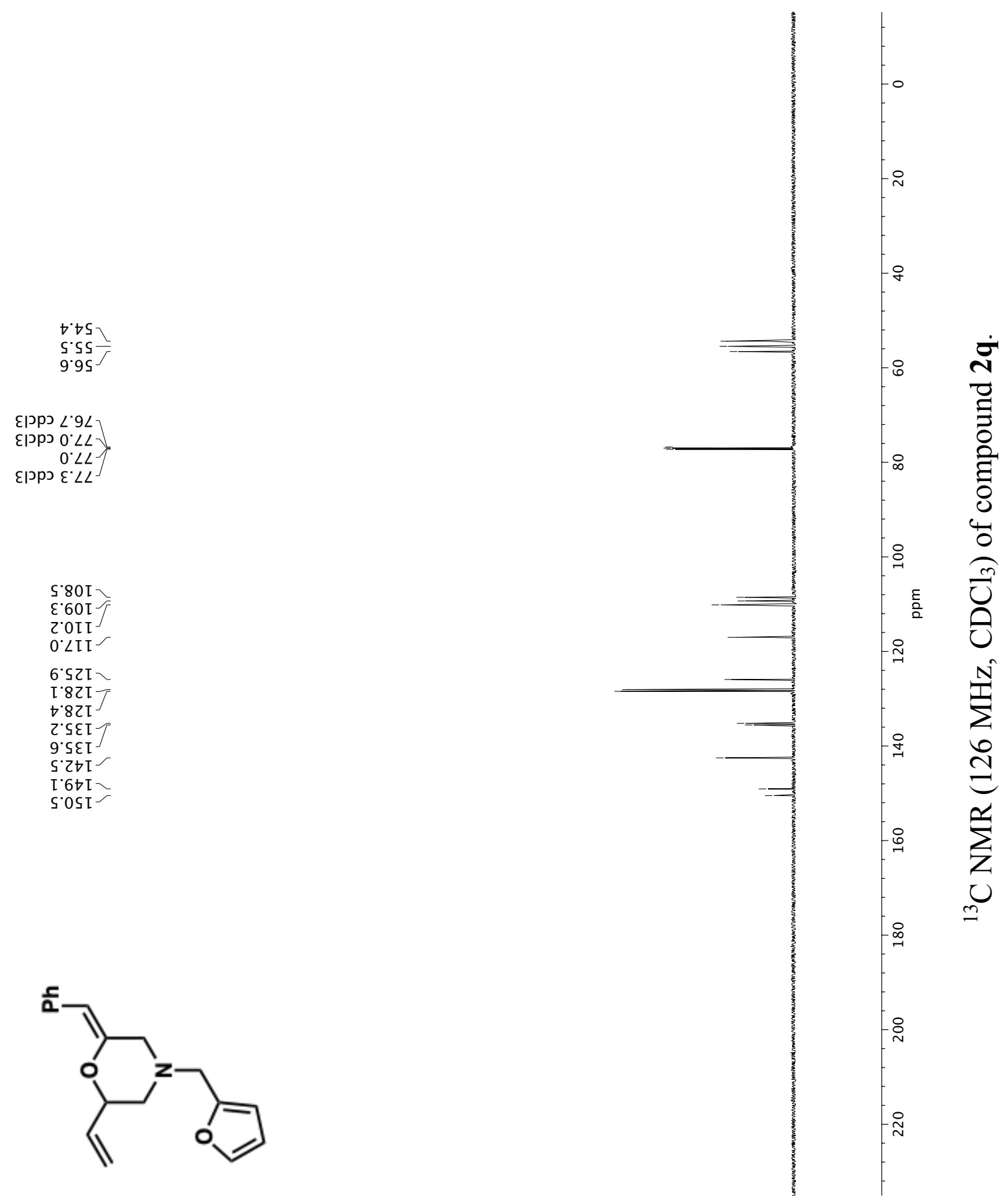

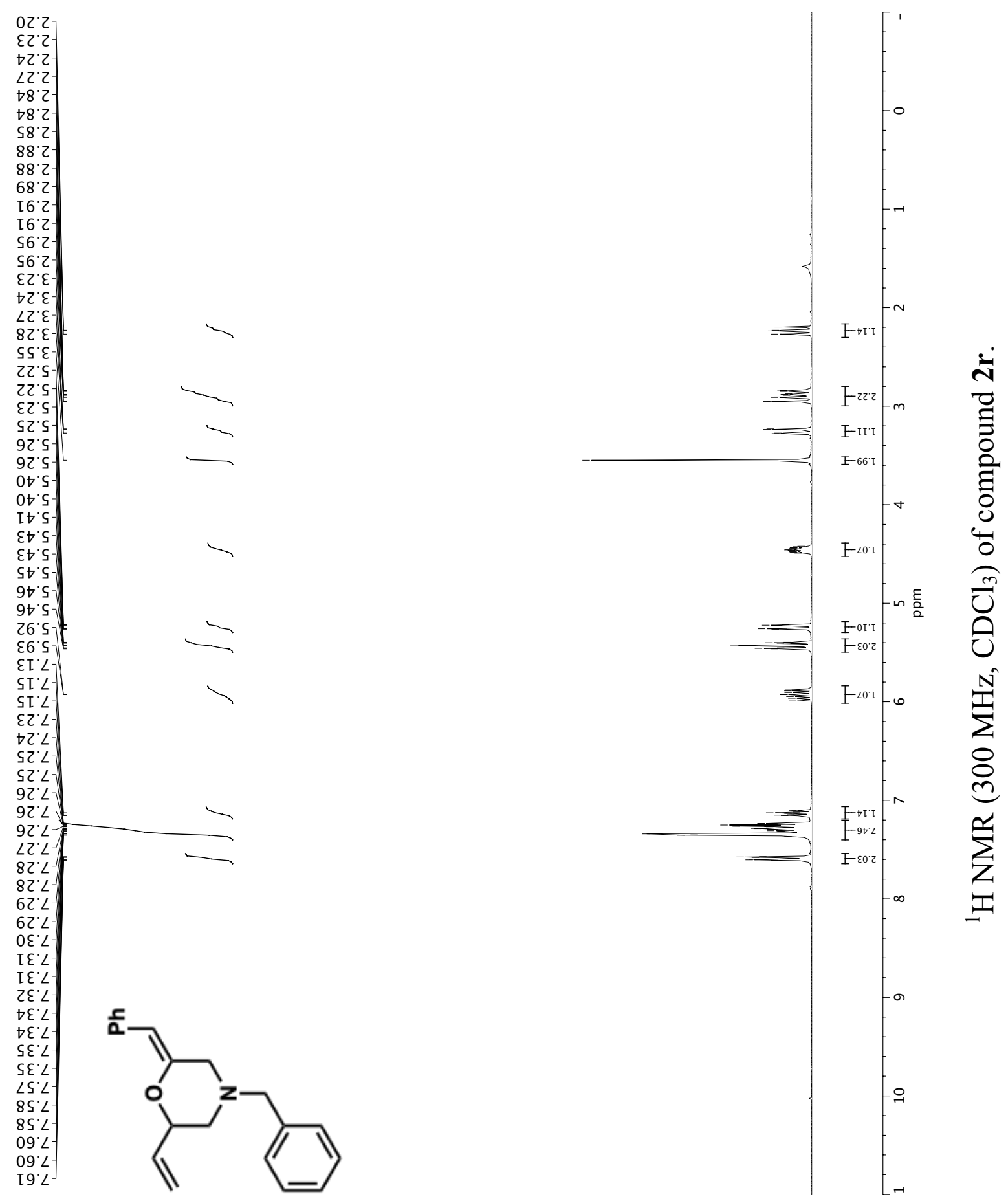

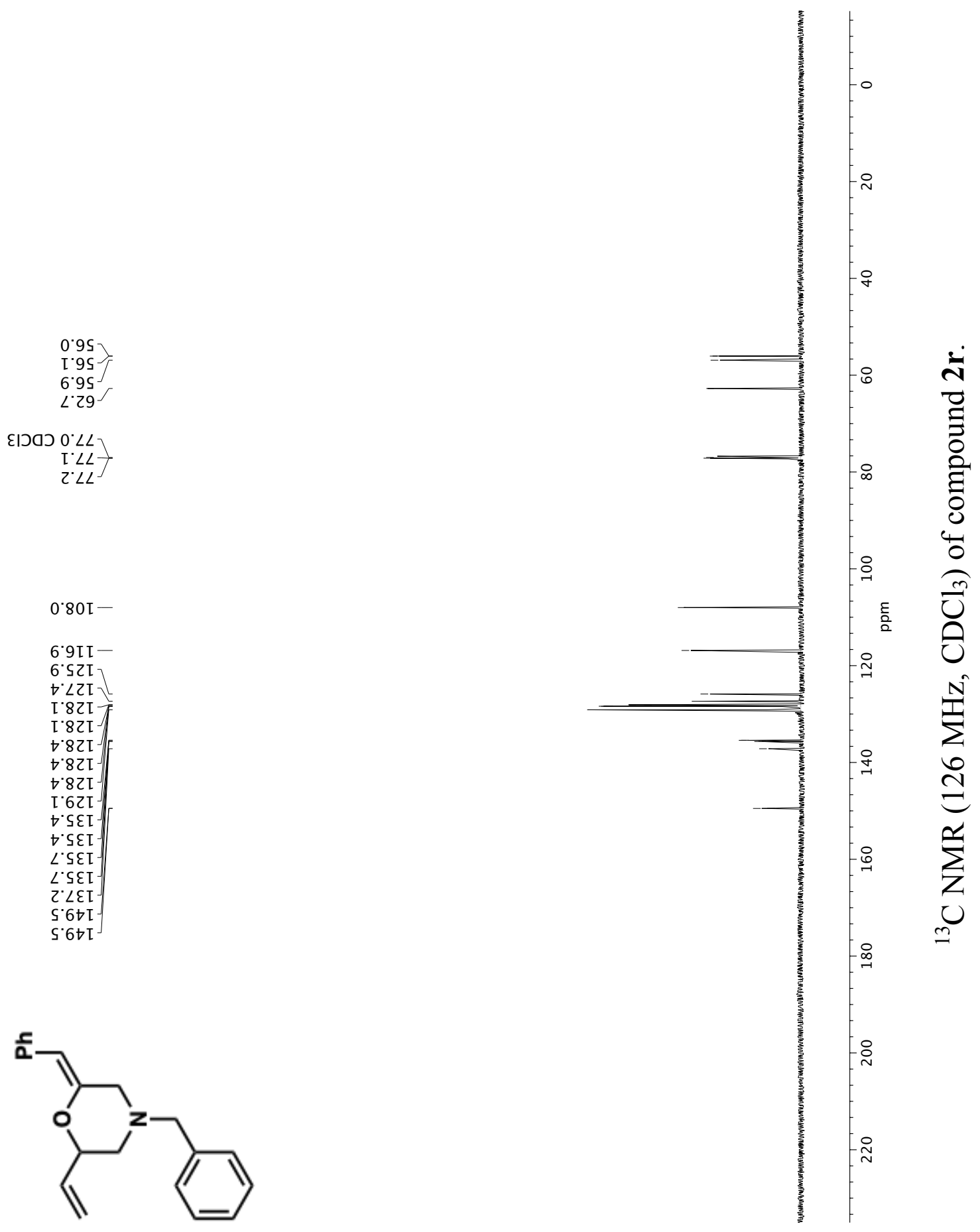
SI-103
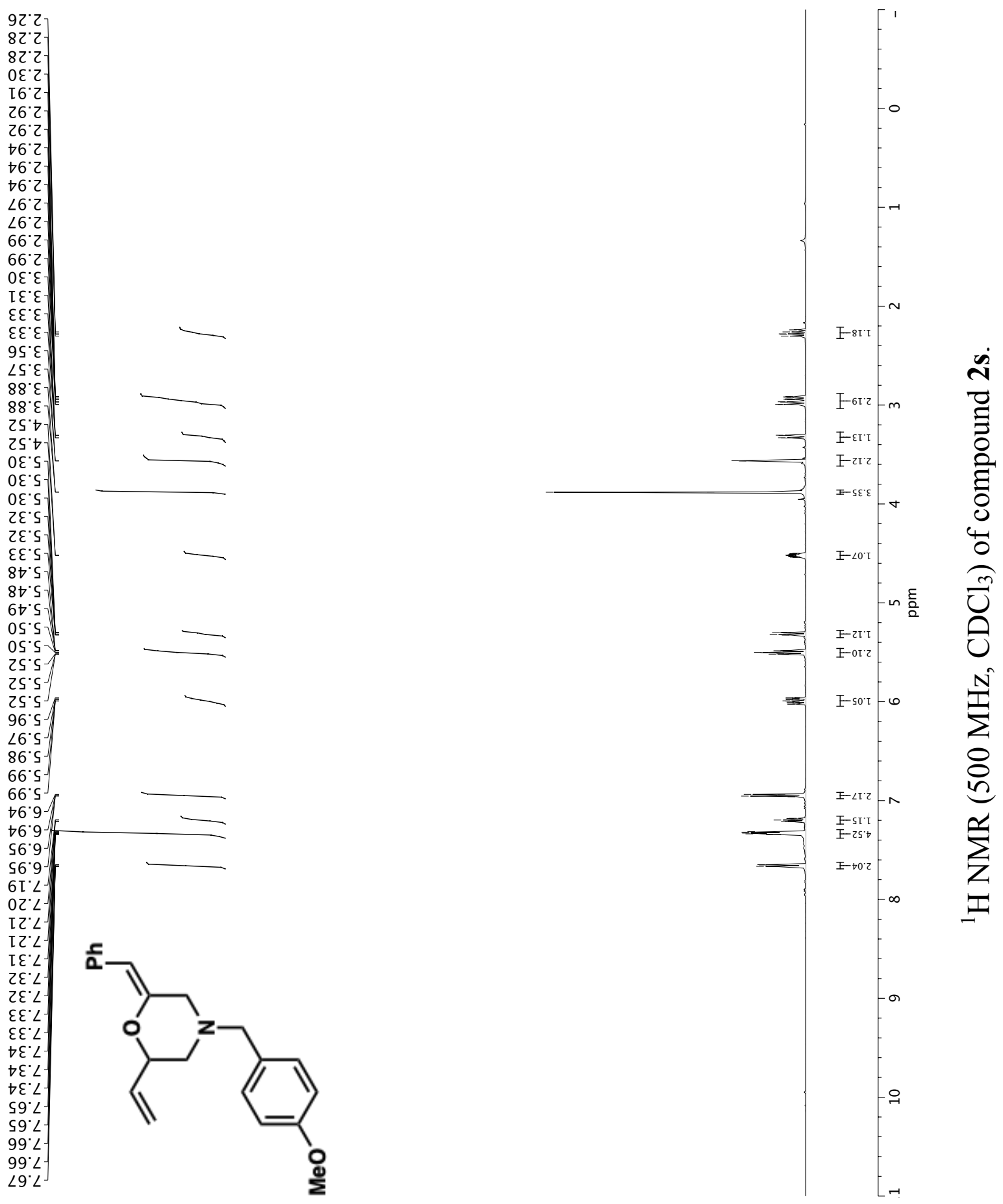

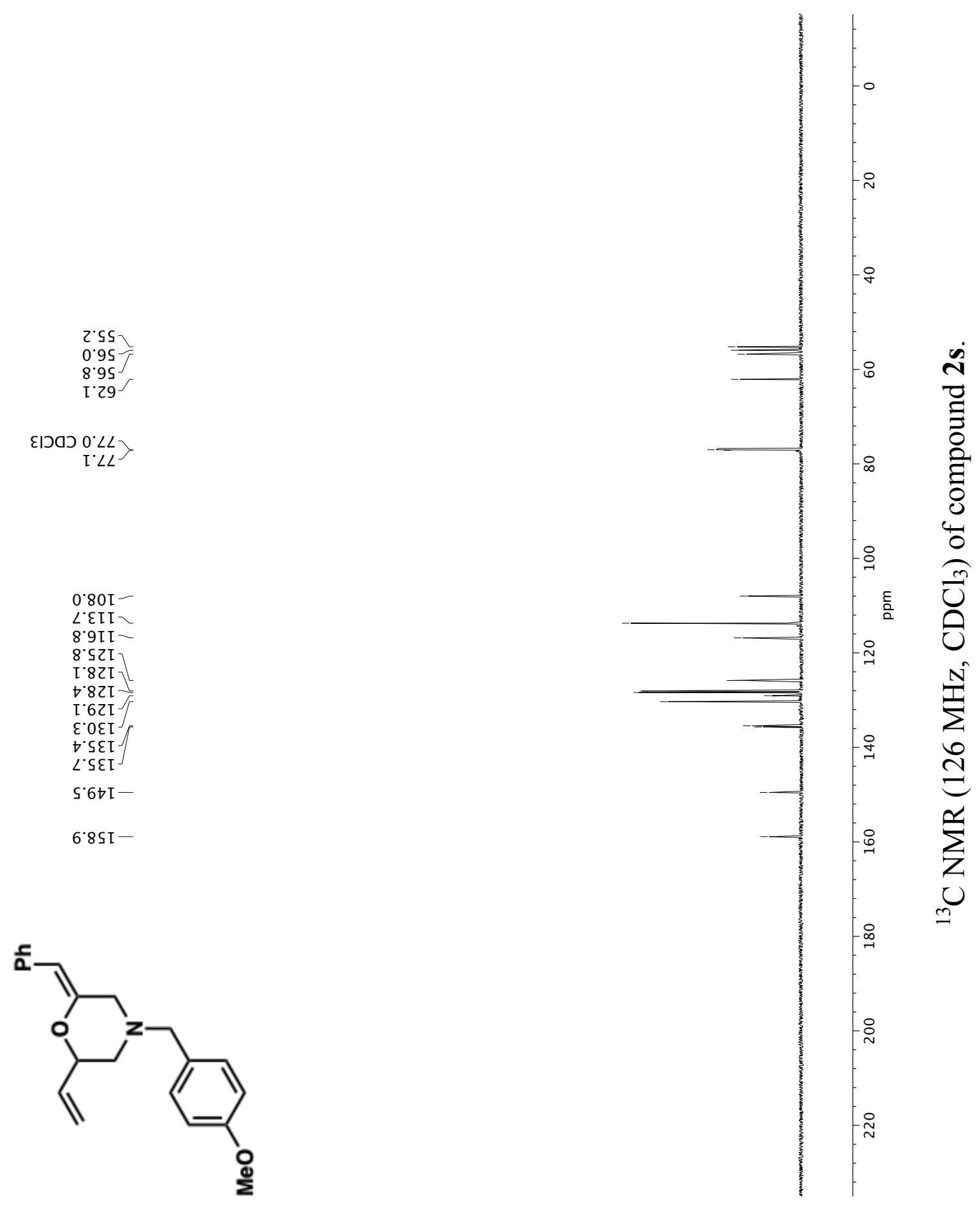


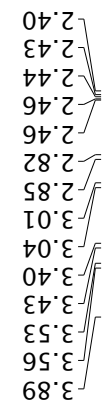

$6 S^{\circ} \mathrm{S}$

[9.5

29.5

ह9. $\mathrm{s}$

76.5

$92^{\circ} \mathrm{L}$

$82^{\circ} \mathrm{L}$

$82^{\circ} L$

$62^{\circ} L$

$62^{\circ} \mathrm{L}$

०६: $L$

$\nabla \varepsilon 2$

$\downarrow \varepsilon ' L$

S $\varepsilon^{\circ} L$

$\varsigma \varepsilon^{\circ} L$

$9 \varepsilon^{\circ} L$

$9 \varepsilon^{\circ} L$

$\angle \varepsilon^{\circ} L$

$\angle \varepsilon^{\circ} L$

$L t^{\circ} L$
$\varepsilon \nabla^{\circ} L$

$\varepsilon \nabla^{\circ} L$

$\varepsilon \forall 2$

$\forall \nabla^{\circ} L$
$\forall \nabla^{\circ} L$

웅

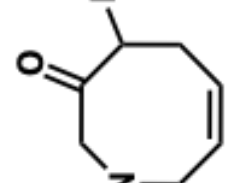

ֻ’

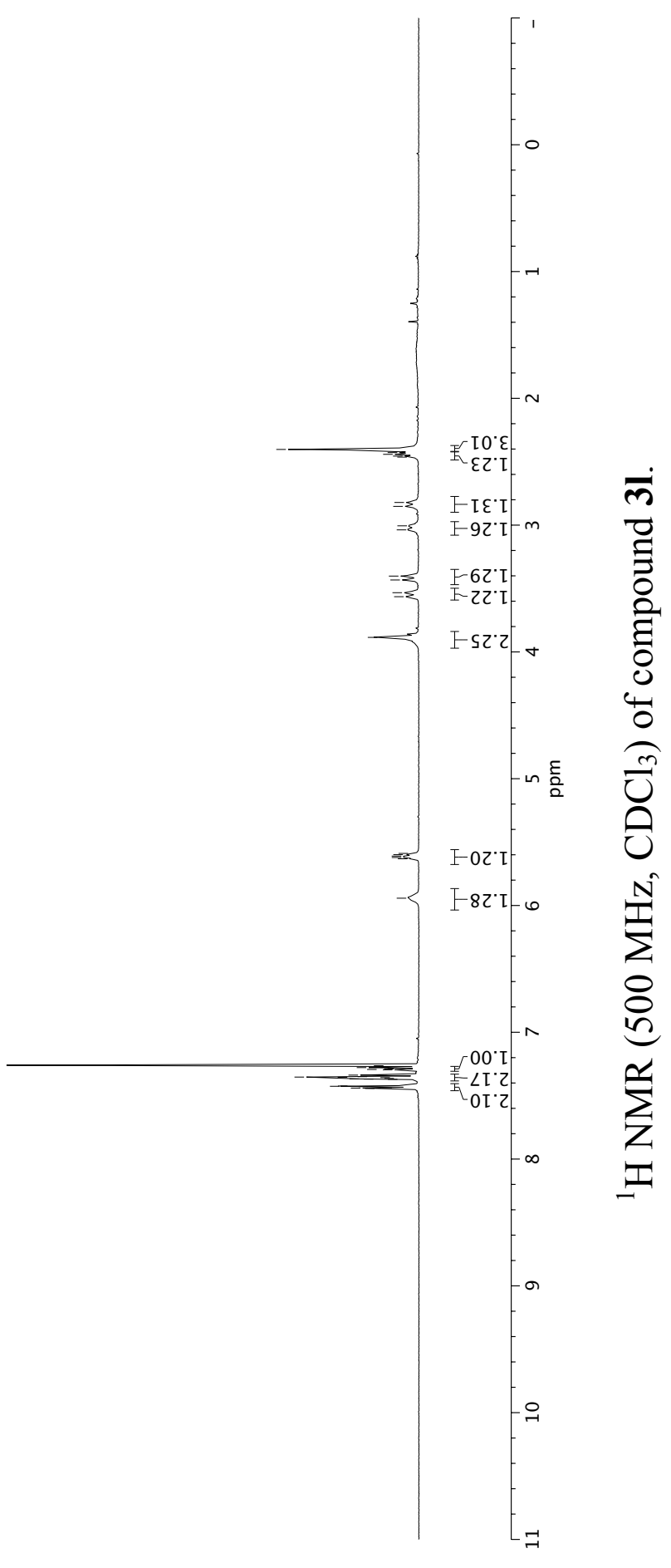




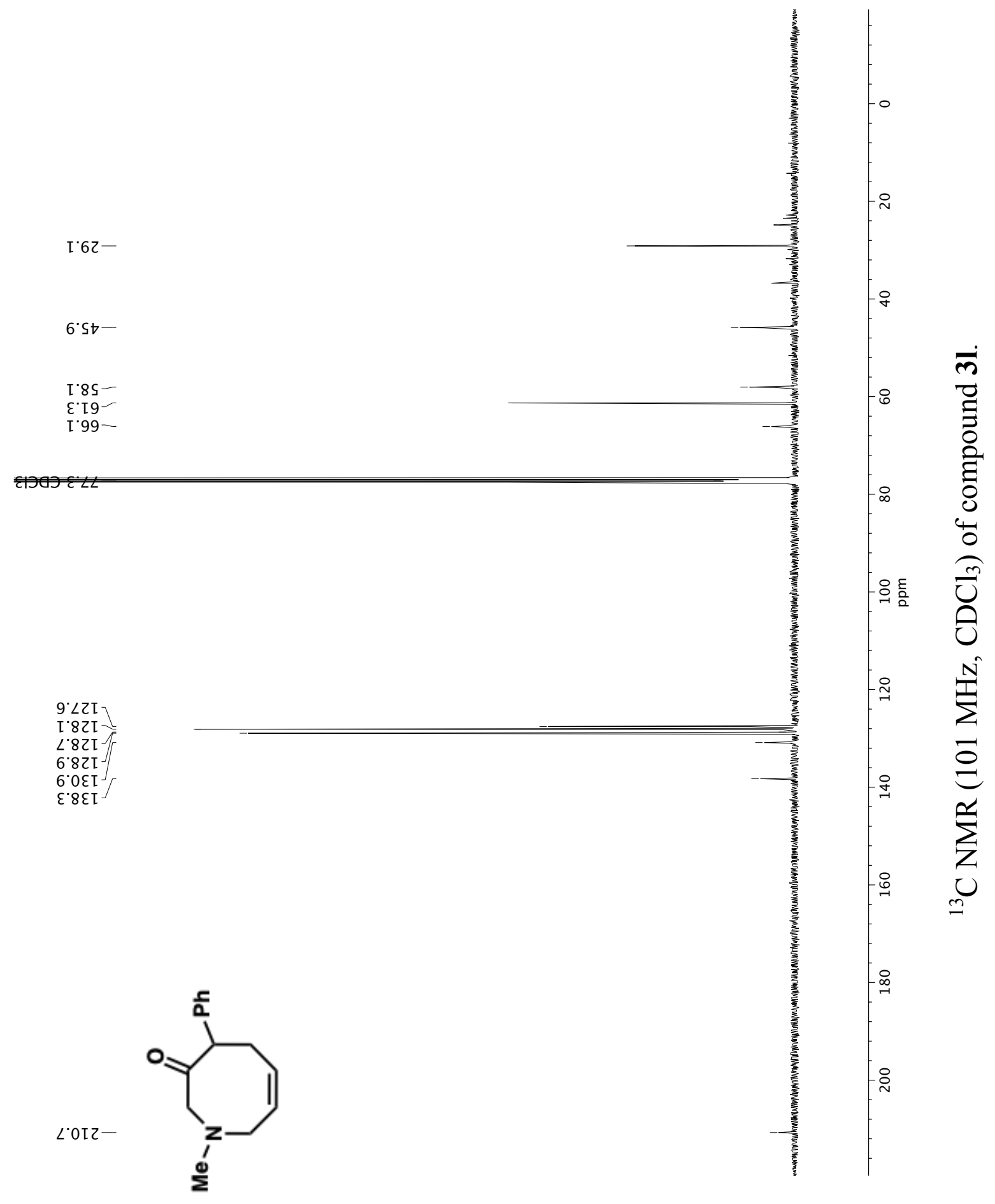


SI-107
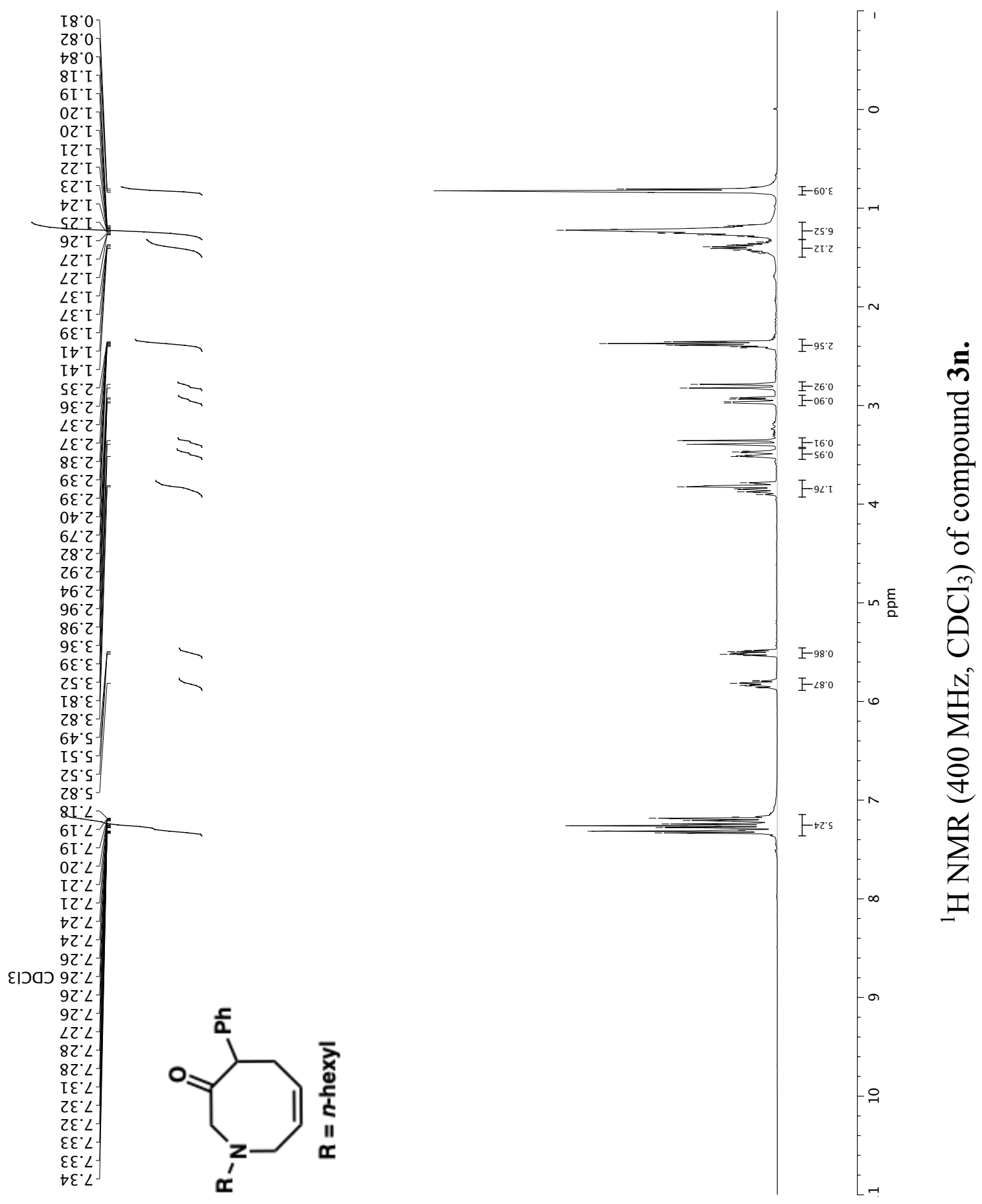
$0 . t \mathrm{I}-$

$9 \cdot 22$

$0<2$

I $\angle 2$

$9^{\circ} 82^{\prime}$
$\angle$.

9.ऽ

$\forall \angle S$

$s .09$

$\forall+79$

ع 0 ว $0<-$

$\varepsilon^{\prime} \angle Z T$

$8 \angle 2 \mathrm{I}$

I.82 I

$9.8 \mathrm{Z}$ -

$2 \cdot 0 \varepsilon \mathrm{I}$

I. $8 \varepsilon \mathrm{I}-$

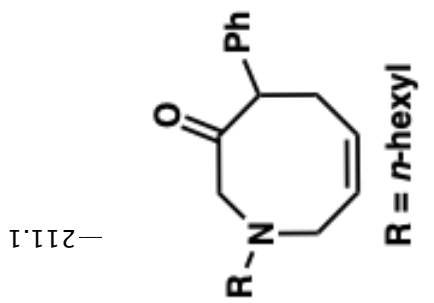

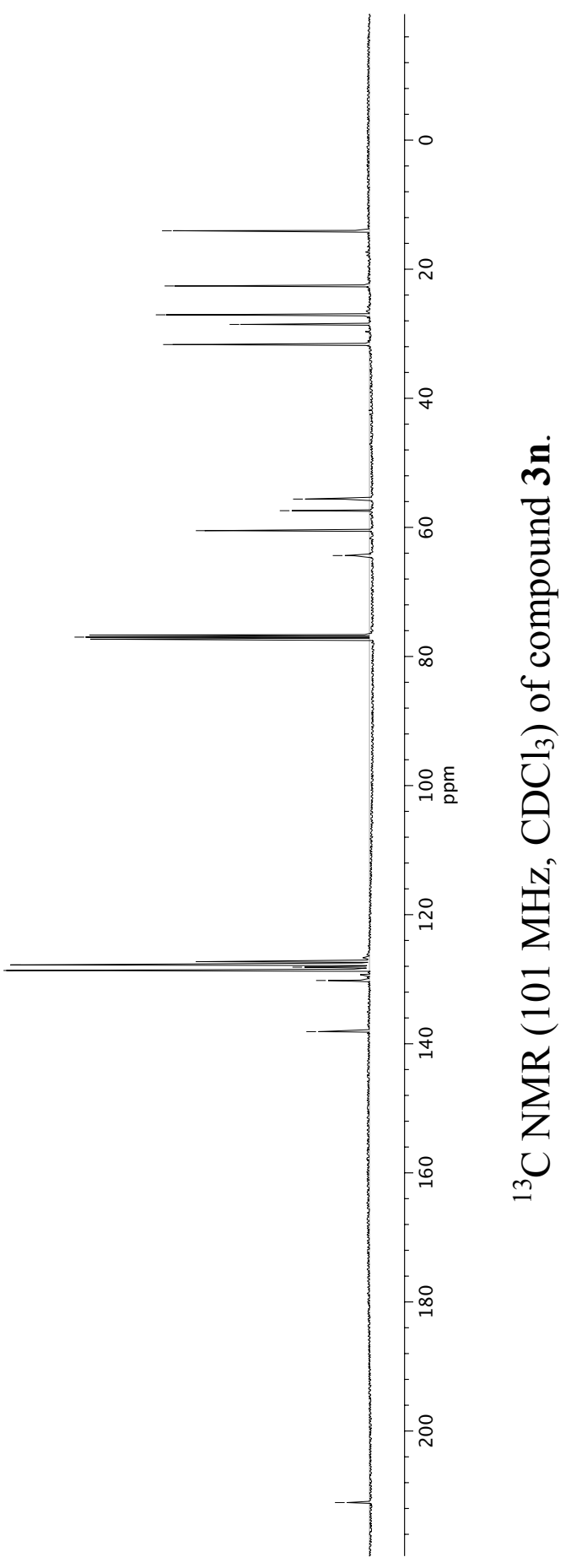



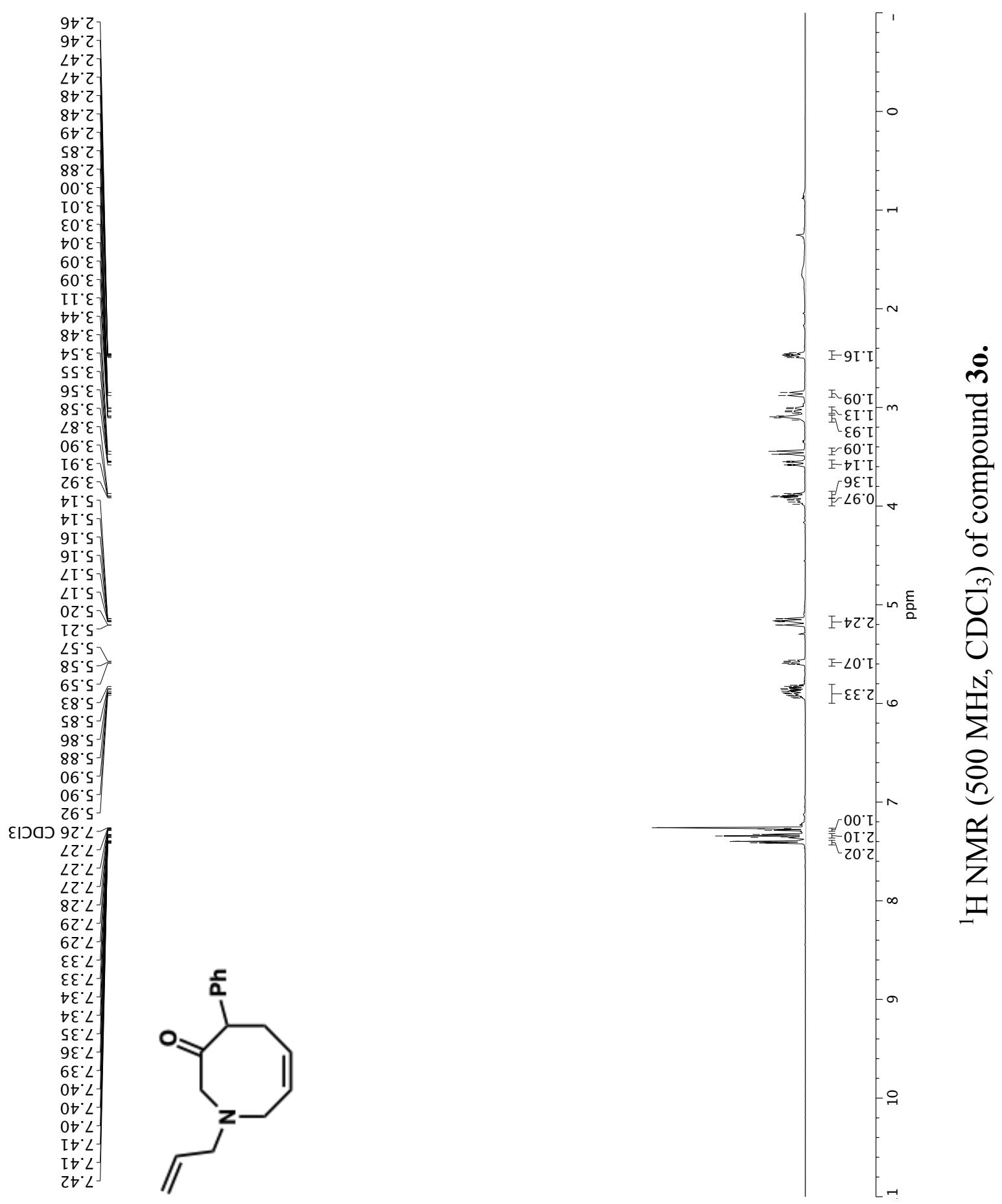
SI-110

$\angle \cdot 8 Z-$

9.SS-

S.09-

6. 69

عدכه 0.LL-

9.8I I-

$\varepsilon \angle Z I$

$8 . \angle 2 I-$

I. $82 \mathrm{I}-$

$5.82 \mathrm{I}$

$\angle 8 Z \mathrm{I}$

乙०0द I

$8 . \sqcup \varepsilon \mathrm{L}$

I. $8 \varepsilon \mathrm{I}$
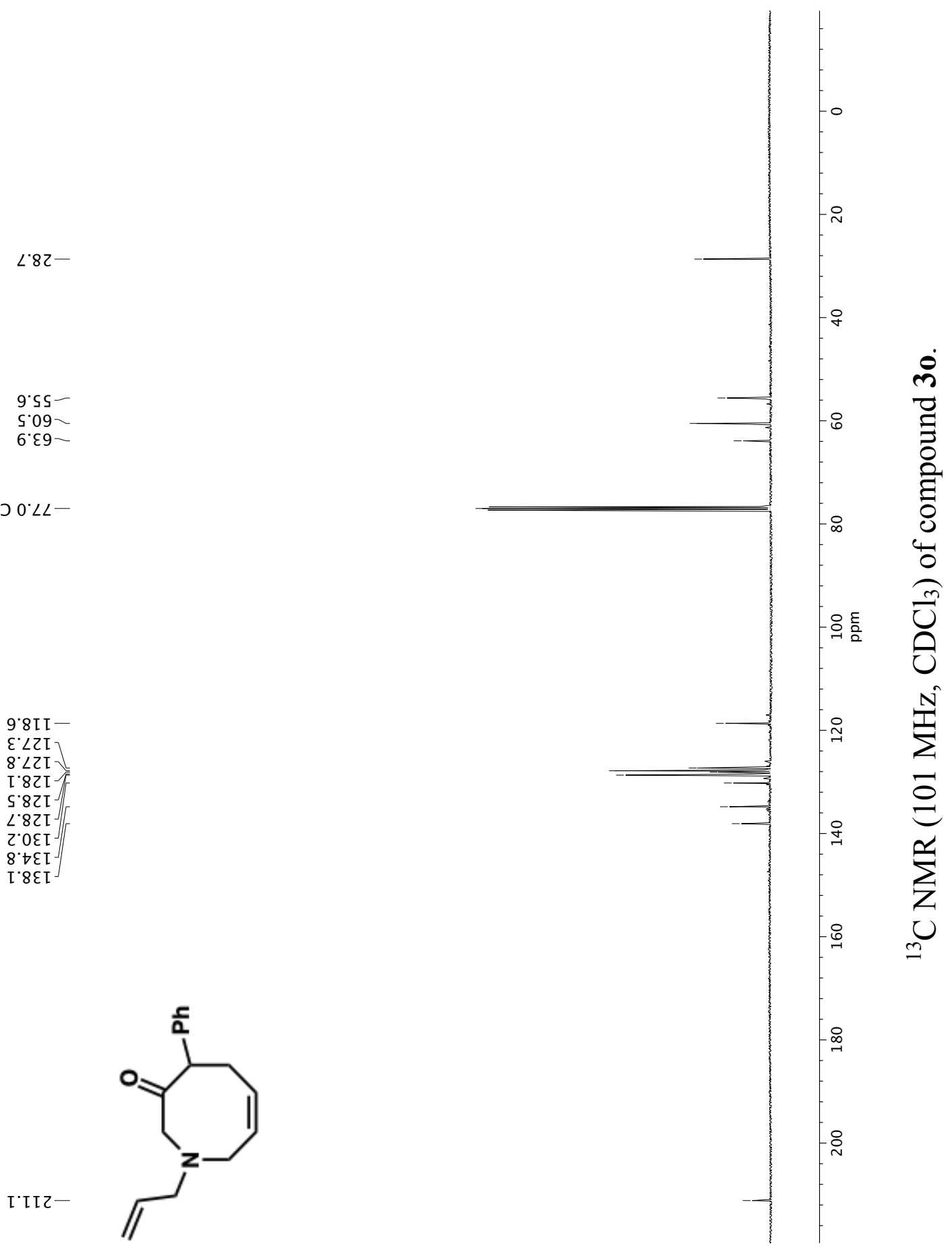

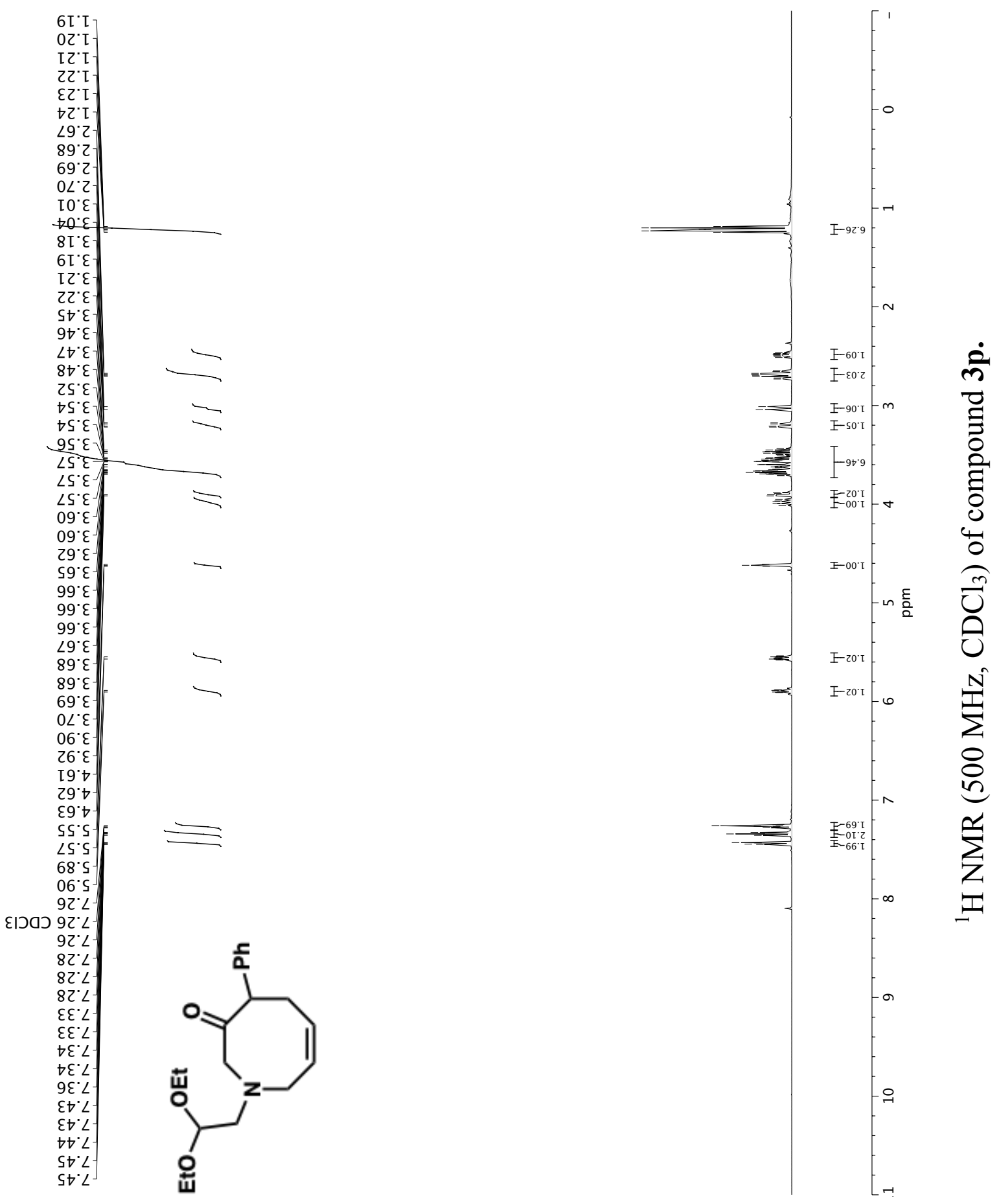

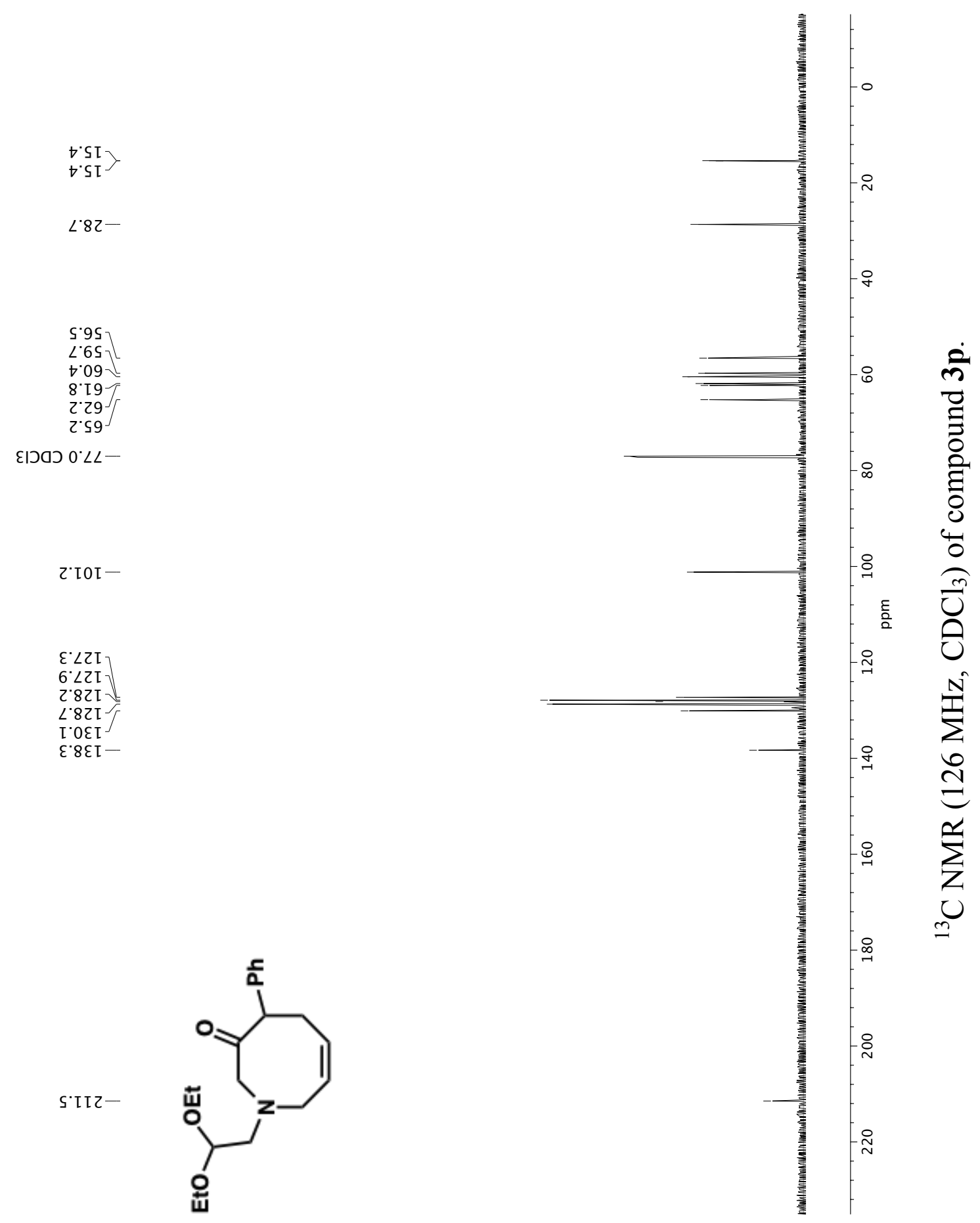

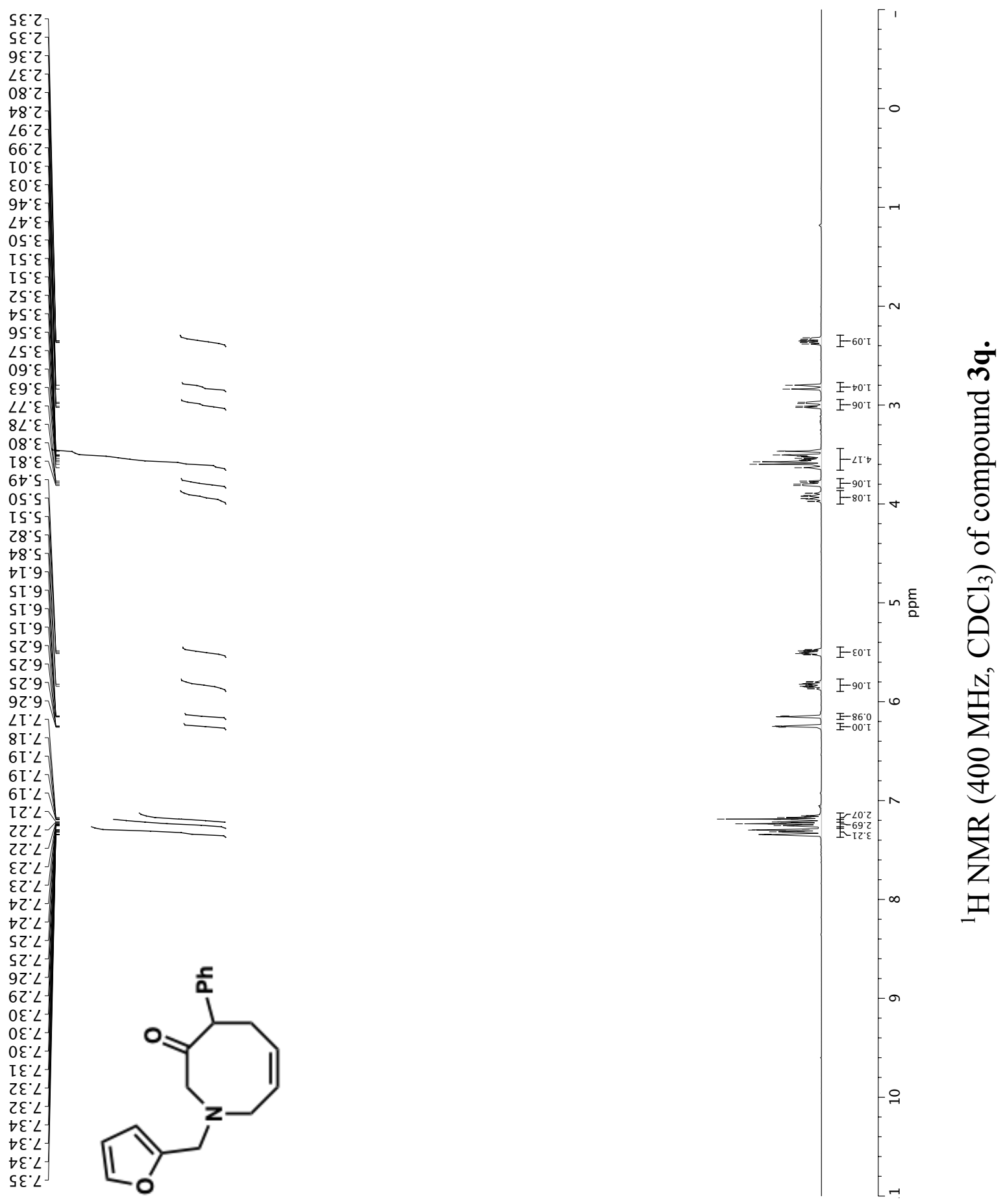
SI-114

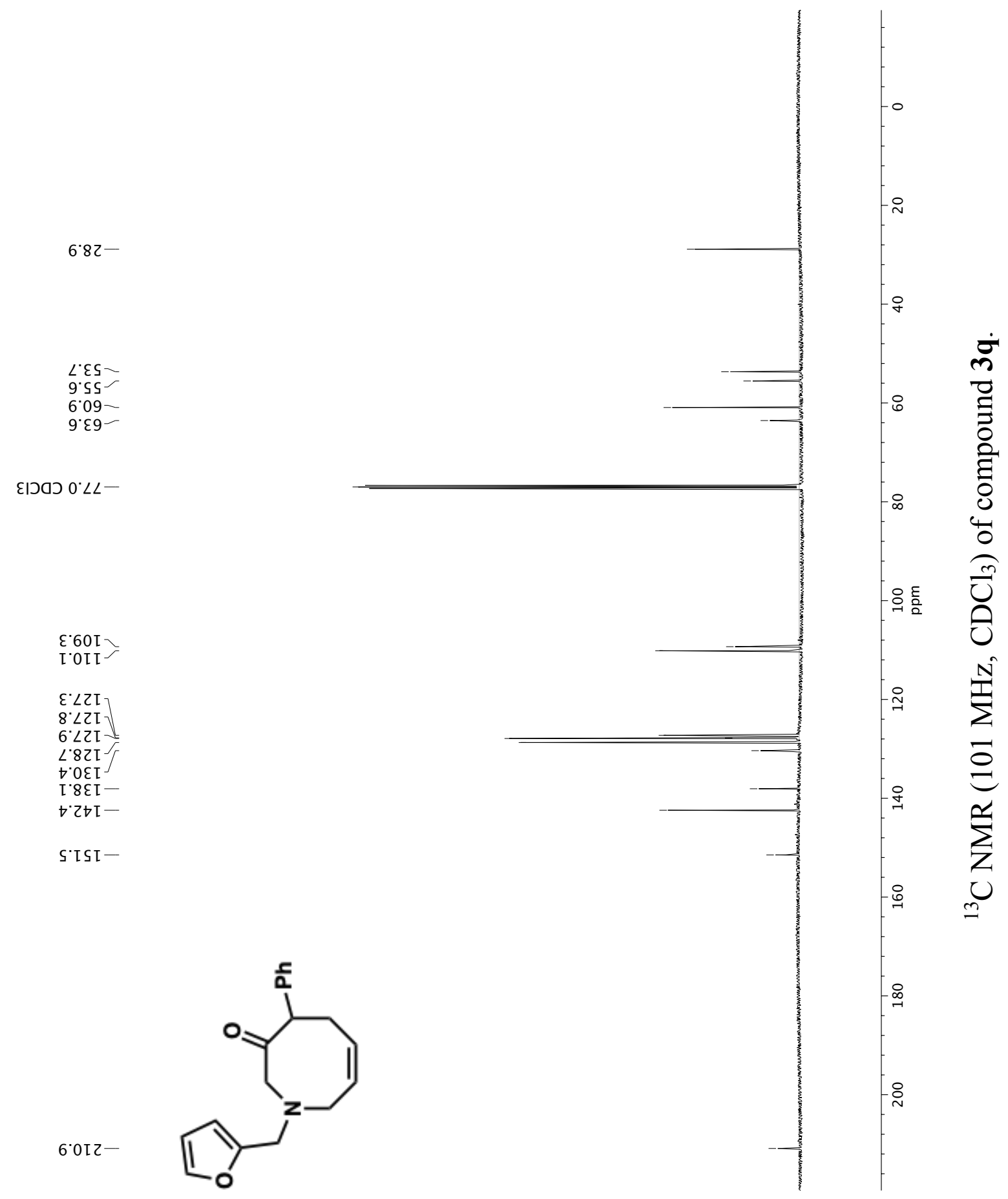



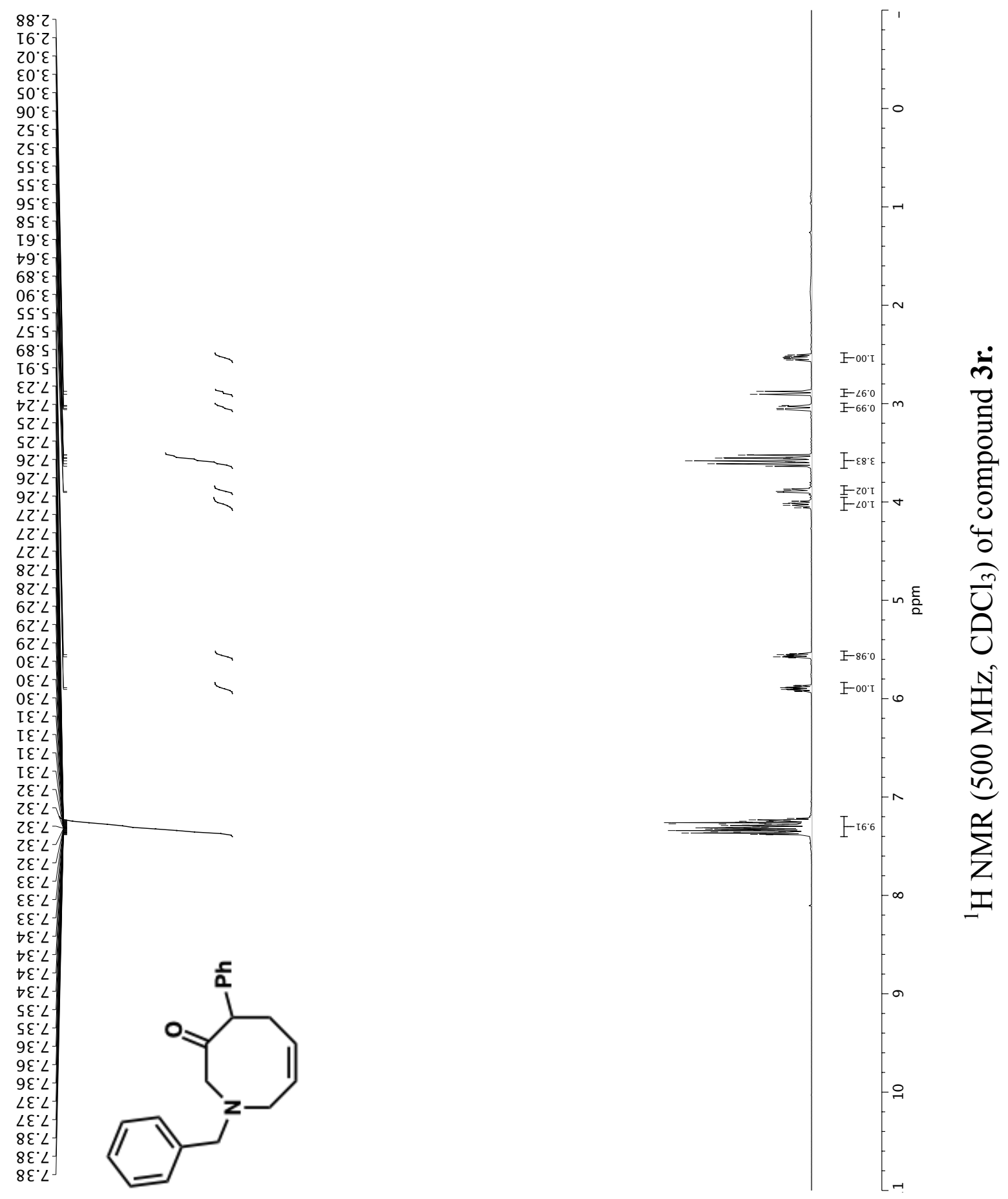

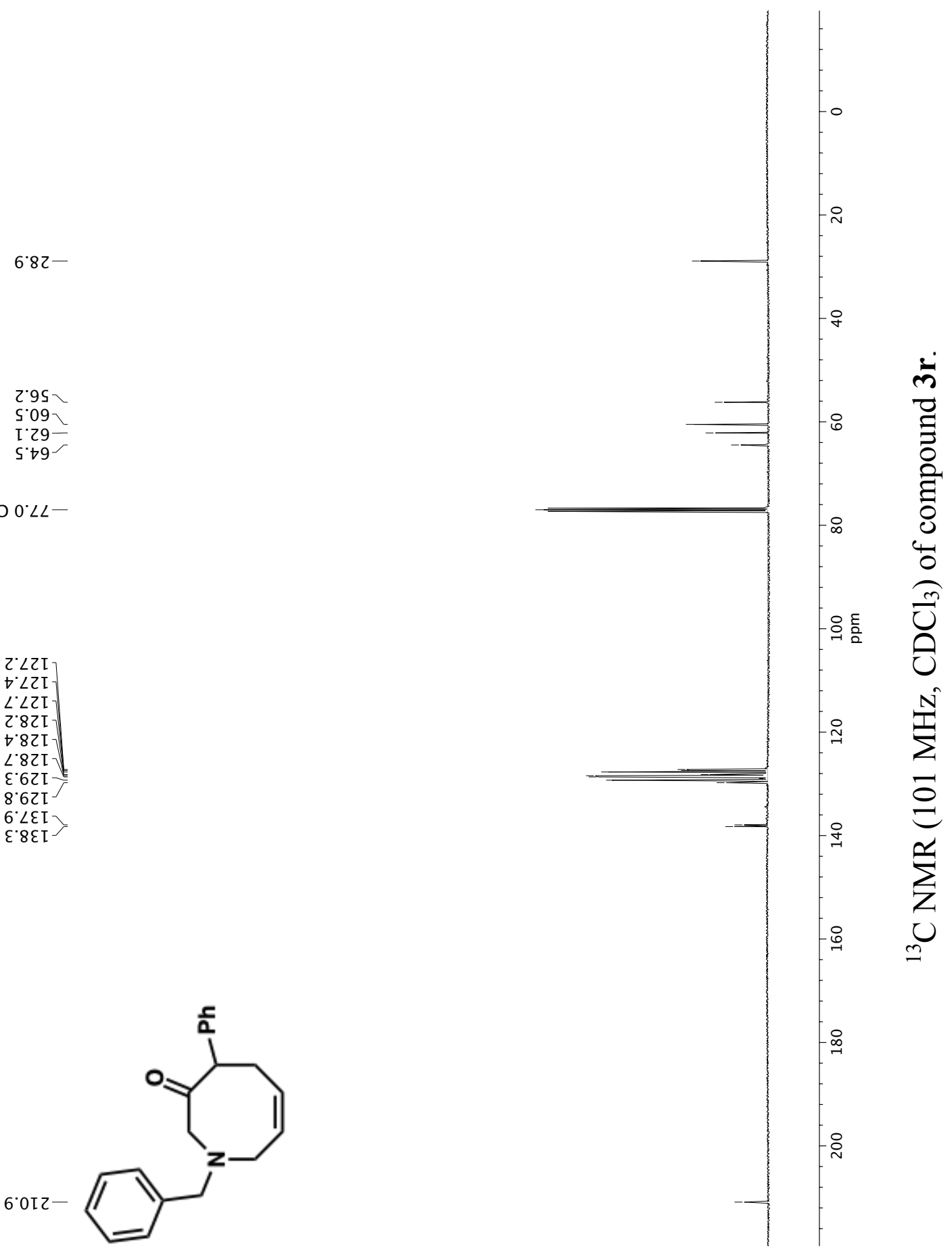

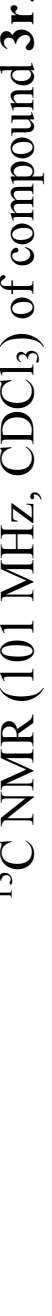



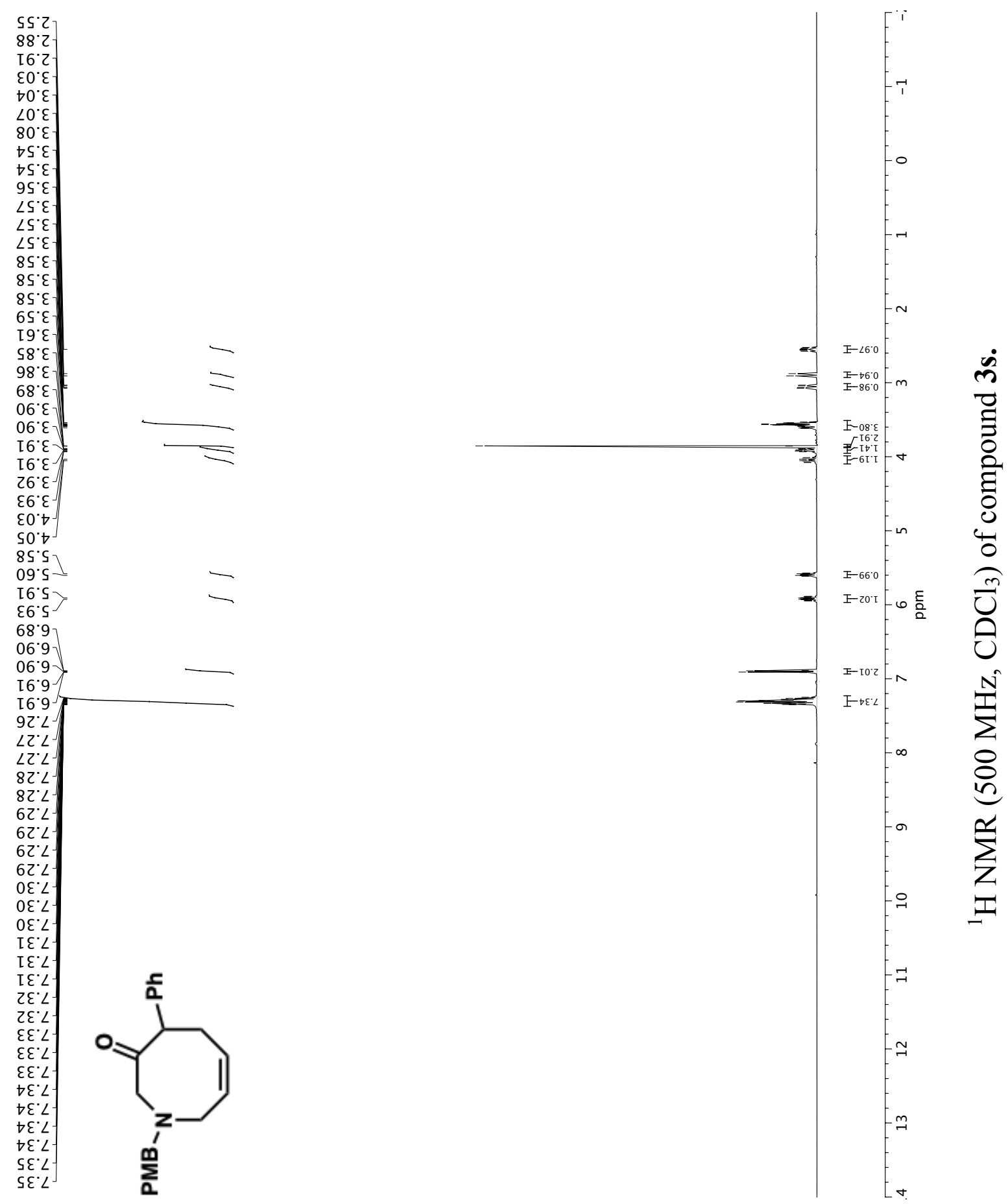
$6.82-$

$$
\begin{aligned}
& \text { E.SS } \\
& \begin{array}{l}
2.95 \\
\text { s.09- }
\end{array} \\
& \text { S.09- } \\
& \rightarrow \forall 9
\end{aligned}
$$

عાدم $0^{\circ} \angle L$

L'EI I-

Z'LZI

$\angle \angle Z I$

$\varepsilon .8 \mathrm{I}$

$9.8 \mathrm{II}$

$9.62 \mathrm{I}$

$0.0 \varepsilon \mathrm{I}-$

$6.8 \mathrm{SI}-$

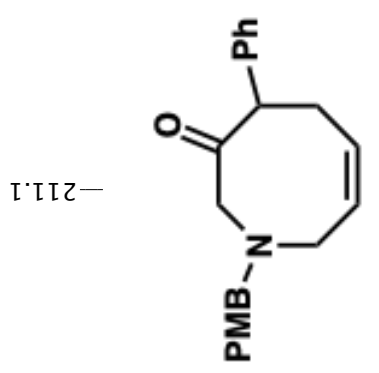

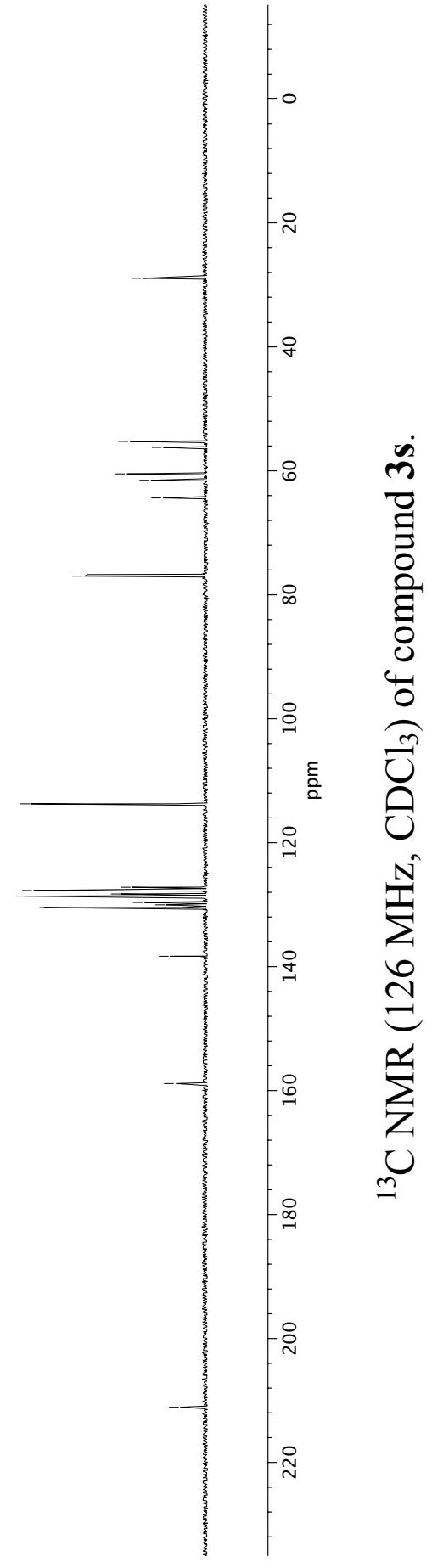

